\title{
Provenance of Syn-rift Clastics in the Eastern Gulf of Mexico: Insight from U-Pb Detrital Zircon Geochronology and Thin Sections
}

\author{
Kory Stuart Wiley
}

Follow this and additional works at: https://researchrepository.wvu.edu/etd

\section{Recommended Citation}

Wiley, Kory Stuart, "Provenance of Syn-rift Clastics in the Eastern Gulf of Mexico: Insight from U-Pb Detrital Zircon Geochronology and Thin Sections" (2017). Graduate Theses, Dissertations, and Problem Reports. 6951.

https://researchrepository.wvu.edu/etd/6951

This Thesis is protected by copyright and/or related rights. It has been brought to you by the The Research Repository @WVU with permission from the rights-holder(s). You are free to use this Thesis in any way that is permitted by the copyright and related rights legislation that applies to your use. For other uses you must obtain permission from the rights-holder(s) directly, unless additional rights are indicated by a Creative Commons license in the record and/ or on the work itself. This Thesis has been accepted for inclusion in WVU Graduate Theses, Dissertations, and Problem Reports collection by an authorized administrator of The Research Repository @ WVU. For more information, please contact researchrepository@mail.wvu.edu. 
Provenance of Syn-rift Clastics in the Eastern Gulf of Mexico:

Insight from U-Pb Detrital Zircon Geochronology and Thin Sections

\section{Kory Stuart Wiley}

Thesis submitted

To Eberly College

At West Virginia University

in partial fulfillment of the requirements

for the degree of

Master of Science

In

Geology

Amy Weislogel, Ph.D., Chair

Jaime Toro, Ph.D.

Kathleen Benison, Ph.D.

Department of Geology and Geography

Morgantown, West Virginia

2017

Keywords: Eastern Gulf of Mexico, U-Pb Detrital Zircon Geochronology, Wood River Formation, Newark Supergroup, South Florida Basin, South Georgia Rift

Copyright 2017 Kory Stuart Wiley 


\author{
ABSTRACT \\ Provenance of Syn-rift Clastics in the Eastern Gulf of Mexico: \\ Insight from U-Pb Detrital Zircon Geochronology and Thin Sections \\ Kory Wiley
}

$\mathrm{U}-\mathrm{Pb}$ detrital zircon geochronology, supplemented by thin section petrography, point counting, and well logs, were used to constrain the provenance of basal Triassic/Jurassic (?) clastic sedimentary rocks in the Eastern Gulf of Mexico (EGOM). Basal clastics were deposited as synrift strata into half grabens present along the EGOM and eastern margin of Laurentia that formed during the initial break up of Pangea. Samples used in this study were collected from the South Florida Basin (SFB) and the South Georgia Rift (SGR). Previous studies of post-rift Jurassic sediment in the Gulf of Mexico has identified a change in source from Laurentian dominated sources in southern Mississippi/Alabama, to Gondwanan dominated sources in the Florida Apalachicola Embayment/offshore. Comparing the provenance of initial syn-rift strata to postrift strata is used to identify initial sediment sources into the EGOM rift basins, and how provenance changed over time. Detrital zircons were collected from five SFB well cutting samples from the Jurassic (?) Wood River Formation and proximal basal sandstones, and six SGR well cutting samples from the Triassic (?) Newark Supergroup, as well as one sample from offshore southern Florida basement. Predominant age populations for the SFB are consistent with a Pan-African origin (525-680 Ma, 1900-2200 Ma). Samples from the SGR are dominated by Laurentian sources (265-330 Ma, 350-420 Ma, 430-490 Ma, 900-1300 Ma), as well as secondary Pan-African sources (525-680 Ma, 1900-2200 Ma). Provenance data is supported by thin section point counting and well logs. Samples in the northern SGR are predominantly derived from Laurentian sources, while the southern samples 3137 and 1854 are a mix of Laurentian and Gondwana signatures. K-S test comparisons of basal sandstones from this study and post-rift Jurassic Norphlet Formation samples from Lovell (2010) and Lisi (2013) show little correlation between samples, implying they do not share similar sources. The exception is the offshore Apalachicola Embayment samples, which indicates that the SGR may have been a potential source for Jurassic sediment in the northern EGOM. The integration of geochronology and petrographic techniques in this study suggests a mix of primarily sedimentary sources from the Suwannee Terranes and metamorphic sources from Laurentia supplied the initial sediment into the EGOM. Results from this study can be used to better constrain tectonic terranes and paleogeography of the early EGOM. 


\section{ACKNOWLEDGEMENTS}

Without the support of my advisors and peers, this project would not have been possible. First and foremost, I would especially like to thank my advisor Dr. Amy Weislogel, who guided me through the toughest parts of my thesis and Master's Degree. I would also like to thank my committee members Dr. Kathleen Benison and Dr. Jaime Toro for their edits and direction. In addition, I would like to thank undergraduates and other graduate students working on the Gulf of Mexico project for providing assistance with sample preparation and analysis, including Sam Bowman, Bethany Royce, Caleb Essex, and Sonnet Gomes.

Monetary support for this project was provided largely by Murphy Oil Corporation, whose support for this project and other projects in the Gulf of Mexico at West Virginia University and University of Alabama has been vital to its success. Secondary funding was provided by the Society for Sedimentary Geology, American Association of Petroleum Geologists, Gulf Coast Association of Geological Societies, and the West Virginia University Shumaker Fund.

Samples and logs for this study were collected from the Florida Geological Survey and Georgia Environmental Protection Division. I would like to thank Jesse Hurd and Paul David at the Florida Geological Survey and Jim Kennedy at the Georgia Environmental Protection Division for helping with sample collection. Offshore Logs studied in this project were collected from the Florida Geological Survey.

Detrital zircon geochronology was completed at the University of South Carolina Center for Elemental Mass Spectrometry. Dr. Barbeau was helpful in sample preparation, running the mass spectrometer, data reduction, and spending long hours dealing with our machine woes.

Lastly, I would like to thank my fellow graduate student friends at WVU, for providing support, comradery, and weekly trips to the Morgantown Brew Pub. I would also like to give a shout out to my friends and family in Arizona. I would especially like to thank my parents, whose moral support helped me see this project to completion. 


\section{TABLE OF CONTENTS}

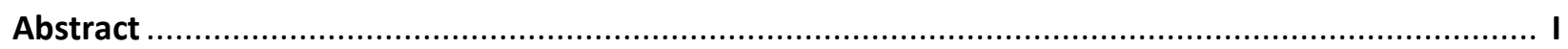

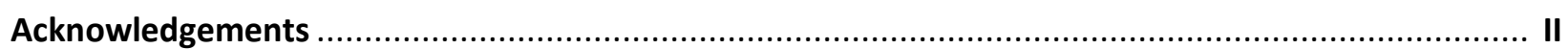

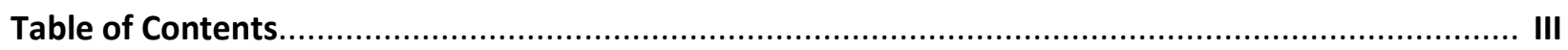

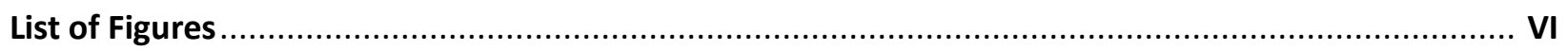

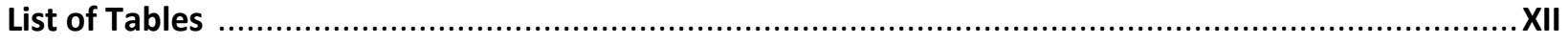

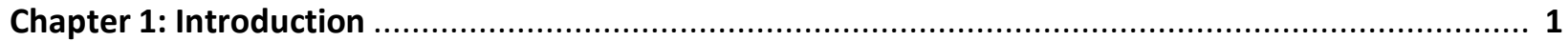

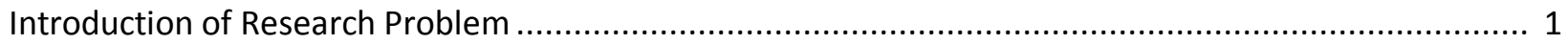

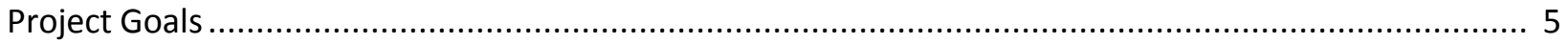

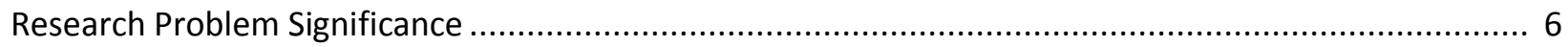

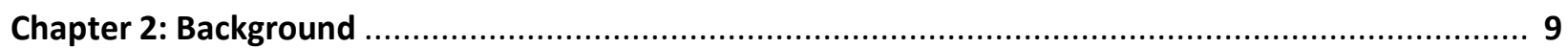

Tectonic Setting and Evolution of the Mesozoic ........................................................................ 9

Basement Rocks: Paleozoic Orogens and Gondwanan Suwannee Terrane ....................................... 13

Generalized Mesozoic Stratigraphy of the Northeastern Gulf of Mexico ......................................... 16

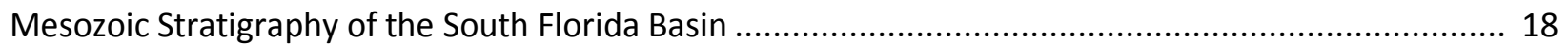

Basement and Mesozoic Stratigraphy of the South Georgia Rift................................................... 20

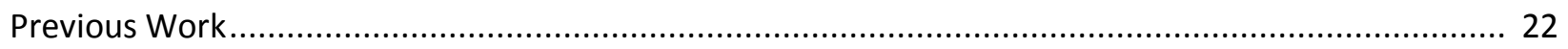

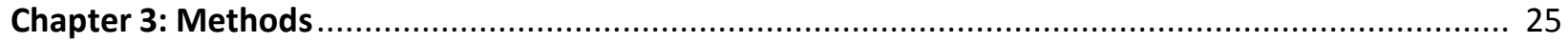

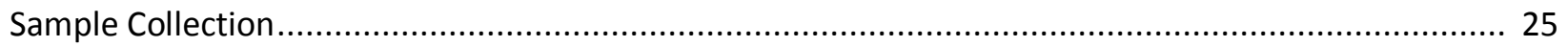

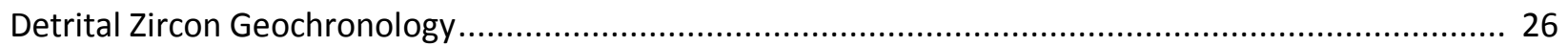

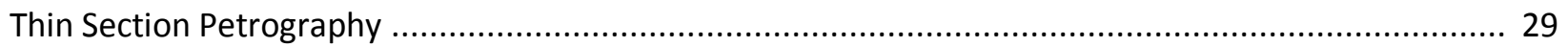

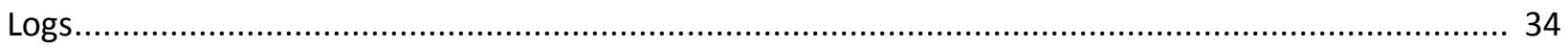

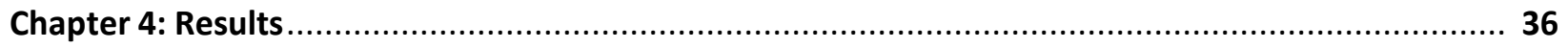

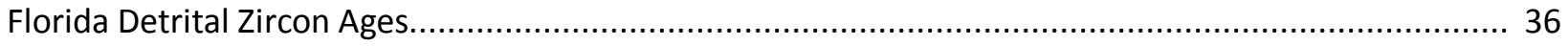


South Florida Composite

South Georgia Rift Detrital Zircon Ages.

South Georgia Rift Composite

Maximum Depositional Age.

Correlation Between Basins.

Thin Sections: South Florida

ODP 68

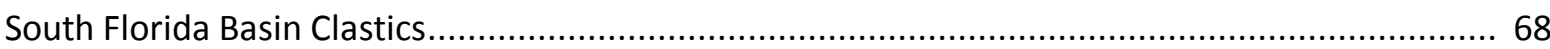

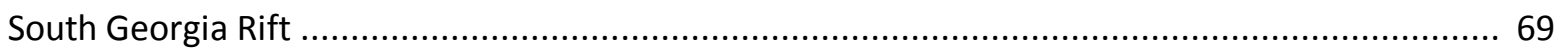

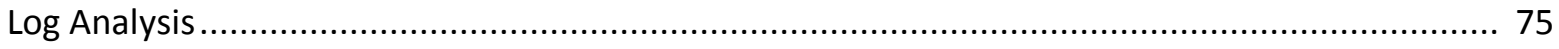

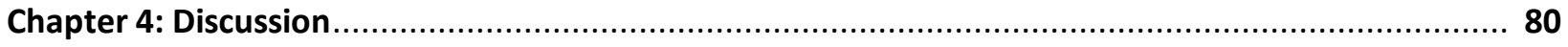

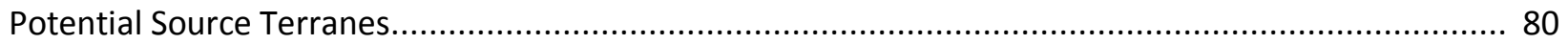

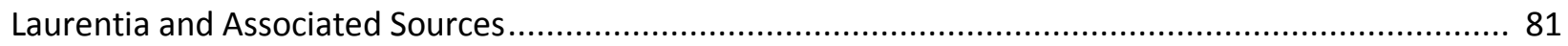

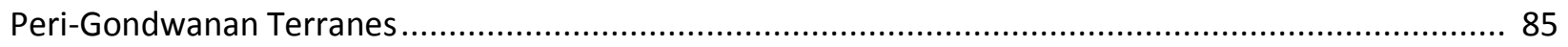

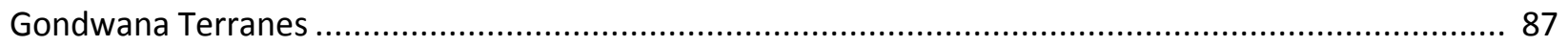

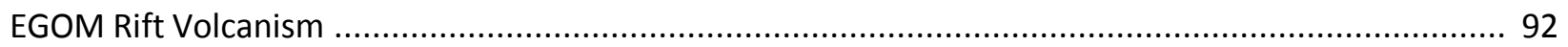

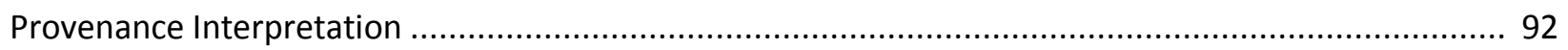

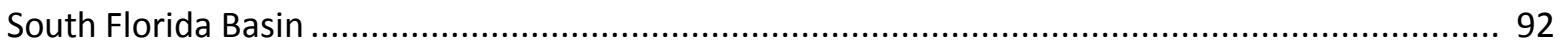

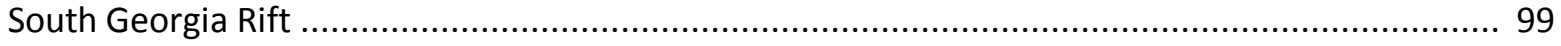

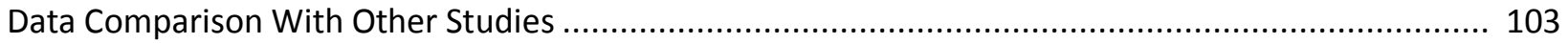

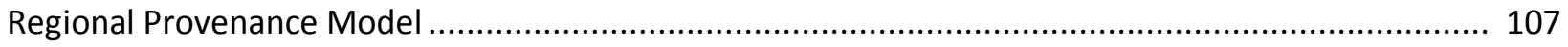

Paleogeographic Interpretations and Regional Provenance Model ............................................. 114

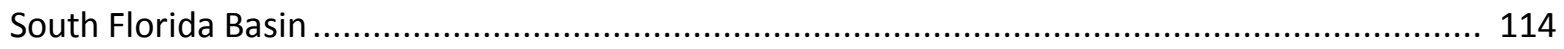

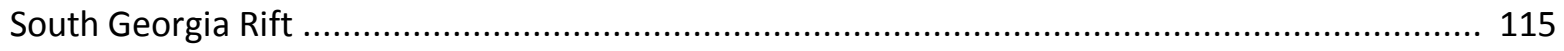


Triassic/Jurassic Paleo-Transport Systems of Laurentia .................................................... 117

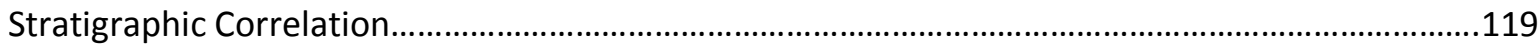

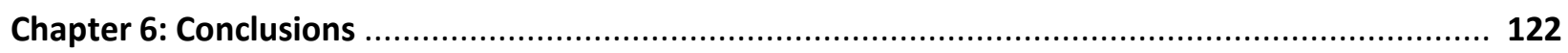

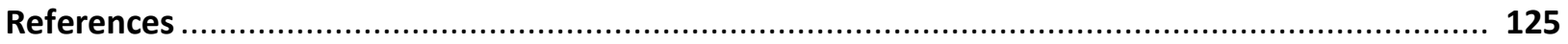

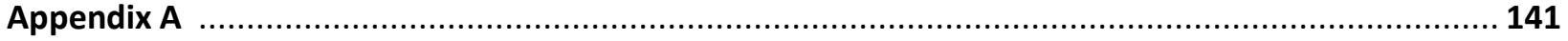

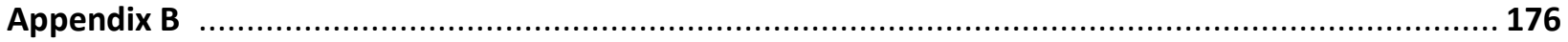

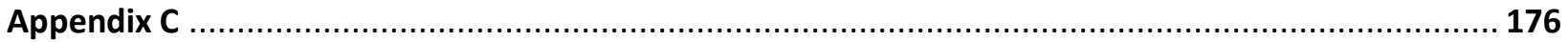




\section{LIST OF FIGURES}

Figure 1. Location of the SFB and SGR in the EGOM. Local structural highs and lows are also identified. Samples collected for this study are listed as Basement, Jurassic-Cretaceous, and Wood River. Previous samples collected from the Norphlet and other offshore clastics are also displayed from Lovell (2010) and Lisi (2013). Structures from Neathery and Thomas (1975); Christenson, (1990); Pollastro et al. (2001). Location of the SGR from Chowns and Williams (1983); Suwannee Suture from Mueller et al. (2014)

Figure 2. Reconstruction of the Gulf of Mexico, with initial separation of Yucatan from Laurentia, and early initiation of the Caribbean during the Middle Jurassic (170 Ma). Modified from Iturralde-Vinet (2003) and Hine (2013).

Figure 3. Paleogeography in the Gulf of Mexico from the Triassic (230 Ma) and the Jurassic (170 Ma). Modified from Blakey (2016).

Figure 4. Location of the various SGR sub-basins, modified by Heffner (2013). SGR sample locations from this study are shown.

Figure 5. Model for rifting and seafloor spreading and rifting from Hudec et al. (2013). A: 210 Ma, initial rifting of Yucatan from Laurentia. B: $163 \mathrm{Ma}$, initial deposition of Louann salt in the GOM following rifting. C: End of salt deposition south of Texas. D: $155 \mathrm{Ma}$, continued extension of crust and salt deposits, E: $149 \mathrm{Ma}$, seafloor spreading begins. F: Modern extent of the GOM, salt, and ocean crust. .. 13

Figure 6. Stratigraphy of the EGOM. Modified from Mancini et al. (1985). Ages from Cohn et al. (2013).

Figure 7. Stratigraphy of the South Florida Basin. Modified from Pollastro et al. (2001). 18

Figure 8. Cross-section of the South Florida Basin. The South Florida Basin is dominated by thick Cenozoic and Cretaceous carbonates. A thin, Triassic/Jurassic clastic layer rests above interpreted basedment. Modified from Pollastro et al. (2001).

Figure 9. Potential provenance model for the Norphlet Formation in the EGOM. Arrows indicate sediment transport directions for the Norphlet in the EGOM. Two dominant source systems were identified for the Norphlet, a W-western system and the E-eastern system. In addition, sediment may also have been derived from the Ar-Arkoma Basin, BW-Black Warriror Basin, and Ap-Appalachian Basin.

From Weislogel et al. (2015).

Figure 10. Location of samples collected from Florida. Samples 778, 10566, and 15095 are from the Wood River Formation. Sample 3578 likely represents correlative facies of the Wood River Formation, or Jurassic-Creatceous transgressive strata. Sample 2012 was collected from central Florida strata of Paleozoic or Jurassic/Cretaceous origin. ODP was collected from offshore basement. No zircons were recovered from 12696, 966, or 1411.

Figure 11. Sample 15095, collected from rock chips, is composed of coarse-grained sandstone and limestone. 
Figure 12. PDP (black line) and KDE (blue) from sample 15095. The $X$ axis is age (Ma), and the $Y$ axis is number of grains.

Figure 13. Sample 3578, collected from cuttings, is composed of coarse grained sand, limestone and shale.

Figure 14. PDP (black line) and KDE (blue) from sample 3578. The $X$ axis is age (Ma), and the $Y$ axis is number of grains.

Figure 15. Sample 778, collected from cuttings, is composed of mixed anhydrite, sand, and limestone. 39 Figure 16. PDP (black line) and KDE (blue) from sample 3578. The $X$ axis is age (Ma), and the $Y$ axis is number of grains.

Figure 17. Sample 10566, collected from cuttings, is composed mainly of coarse grained shale, sandstone, and limestone.

Figure 18. PDP (black line) and KDE (blue) from sample 10566. The $X$ axis is age (Ma) and the $Y$ axis is the number of grains.

Figure 19. Sample 2012, collected from cuttings, is composed mainly of coarse grained shale and sandstone.

Figure 20. PDP (black line) and KDE (blue) from sample 2012. The $X$ axis is age (Ma), and the $Y$ axis is number of grains.

Figure 21. Sample collected from interval 22-24.5, composed of phylite. Core photo provided from ODP online database.

Figure 22. PDP (black line) and KDE (blue) for sample ODP. The $\mathrm{X}$ axis is age (Ma), and the $\mathrm{Y}$ axis is number of grains.

Figure 23. Cumulative PDP and KDE for the SFB (excluding sample ODP). The $X$ axis is age (Ma), and the $Y$ axis is number of grains. The two predominant peaks are $605 \mathrm{Ma}$ and $2080 \mathrm{Ma}$.

Figure 24. Normalized Probability Plot for all samples in South Florida, from north to south. Largest populations range from $\sim 580 \mathrm{Ma}-610 \mathrm{Ma}$ for all samples.

Figure 25. All age peaks for the SFB samples. Boxes correspond to major age populations. Major populations are $240 \mathrm{Ma}-450 \mathrm{Ma}, 500 \mathrm{Ma}-650 \mathrm{Ma}, 700-900 \mathrm{Ma}$ and 2000-2300 Ma.

Figure 26. Ages and $U / T h$ ratios for detrital zircon of the South Florida samples. Metamorphic zircons were identified based on a $\mathrm{U} / \mathrm{Th}$ ratio greater than 5 .

Figure 27. Major age peaks for metamorphic detrital zircon in the SFB (blue boxes). Two groupings are present between 400-800 Ma and 1900-2300 Ma.

Figure 28. Location of the Newark deposits (green) sampled in this study. Other samples localites are Norphlet samples (blue) from Lovell (2010) and Lisi (2013). 
Figure 29. Sample 108, collected from cuttings, composed mainly of quartz, barite, and sandstone fragments.

Figure 30. PDP (black line) and KDE (blue) from sample 108. The $X$ axis is age (Ma), and the $Y$ axis is number of grains. A: Detrital zircons from $0 \mathrm{Ma}$ to $3000 \mathrm{Ma}$. B: Detrital zircons from $0 \mathrm{Ma}$ to $800 \mathrm{Ma}$. C: Detrital zircons from $800 \mathrm{Ma}$ to $3000 \mathrm{Ma}$.

Figure 31. Sample 3113, collected from cuttings and composed mainly of quartz, shale.

Figure 32. PDP (black line) and KDE (blue) from sample 3113. The $X$ axis is age (Ma), and the $Y$ axis is number of grains. A: Detrital zircons from $0 \mathrm{Ma}$ to $3000 \mathrm{Ma}$. B: Detrital zircons from $0 \mathrm{Ma}$ to $800 \mathrm{Ma}$. C: Detrital zircons from $800 \mathrm{Ma}$ to $3000 \mathrm{Ma}$.

Figure 33. Sample 619. Collected from cuttings, and composed mainly of barite, quartz, shale and sandstone.

Figure 34. PDP (black line) and KDE (blue). The $X$ axis is age (Ma), and the $Y$ axis is number of grains. A: Detrital zircons from 0 to $3000 \mathrm{Ma}$. B: Detrital zircons from 0 Ma to $800 \mathrm{Ma}$. C: Detrital zircons from 800 Ma to 3000 Ma.

Figure 35. Sample 3137 Sample was collected from cuttings, and consists mainly of red sandstone and shale, as well as barite.

Figure 36. PDP (black line) and KDE (blue) from sample 3137. The $X$ axis is age (Ma), and the $Y$ axis is number of grains. A: Detrital zircons from 0 Ma to $3000 \mathrm{Ma}$. B: Detrital zircons from 0 Ma to $800 \mathrm{Ma}$. C:

Detrital zircons from $800 \mathrm{Ma}$ to $3000 \mathrm{Ma}$.

Figure 37. Sample 442. Sample was collected from cuttings/rock chips, and consists mainly of coarse grained sandstone and shale.

Figure 38. PDP (black line) and KDE (blue) from sample 442. The $X$ axis is age (Ma), and the $Y$ axis is number of grains A: Detrital zircons from 0 to $3000 \mathrm{Ma}$. B: Detrital zircons from $0 \mathrm{Ma}$ to $800 \mathrm{Ma}$. C: Detrital zircons from $800 \mathrm{Ma}$ to $3000 \mathrm{Ma}$.

Figure 39. Sample 1854. Sample was collected from cuttings/rock chips, and consists of coarse grained sandstone and shale.

Figure 40. PDP (black line) and KDE (blue) from sample 1854. The $X$ axis is age (Ma), and the $Y$ axis is number of grains A: Detrital zircons from $0 \mathrm{Ma}$ to $3000 \mathrm{Ma}$. B: Detrital zircons from 0 Ma to $800 \mathrm{Ma}$. C:

Detrital zircons from $800 \mathrm{Ma}$ to $3000 \mathrm{Ma}$.

Figure 41. Cumulative PDP (black line) and KDE (blue) for all SGR samples. Major peaks include 297 to $480 \mathrm{Ma}, 509 \mathrm{Ma} 672 \mathrm{Ma}$, and 982 to $1184 \mathrm{Ma}$.

Figure 42. Normalized Probability Plot for the SGR.

Figure 43. Major Age peaks for the SGR. Major age groups of peaks include 200-500 Ma, 525-725 Ma, 750-1100 Ma, 1200-1500 Ma, and 2275-2600 Ma.

Figure 44. Metamorphic zircons for the SGR. Metamorphic zircons were identified based on a U/Th ratio greater than 5. 
Figure 45. Major metamorphic peaks for the SGR. Major groupings are between 200-600, and 900-1400 Ma).

Figure 46. Normalized Probability Plot for the SGR and SFB.

Figure 47. Cumulative Density Plot. The majority of the samples are greater than $90 \%$ younger than $\sim 700$ Ma, aside from sample 2012.

Figure 48. CDP for the SGR. The largest sample differences are present around 500-600 Ma, $900 \mathrm{Ma}$, and 1500-2200 Ma.

Figure 49. QFL plot of the Wood River and proximal clastics in the SFB. Samples fall predominantly in the subarkose range, although sample 12542 is Lithic Arkose. 70

Figure 50. Collected from core. A: ODP-1. Mylonitized quartz veins in a diabase intrusion. B: ODP-1. Quartz veins showing two directions of growth. C: ODP-2. Quartz grain with zircons inclusions. D: ODP2. Calcite veins showing two stage growth: First episode of micritic calcite, second stage blocky. E: ODP2. Igneous fragment surrounded by blocky calcite cement F: ODP-2. Perthite fragment surrounded by calcite cement.

Figure 51. Collected from cuttings. A: 10566. Detrital quartz fragments. B. 10566. Quartz and feldspar (?) fragments. Well sorted, low porosity. C: 10566 . Microcline surrounded by quartz. D: 778 . Detrital quartz fragments. E: 778. Detrital quartz and feldspar (?). F: 778. Detrital quartz and microcline.

Figure 52. Collected from cuttings. A: 12542. Mix of feldspar (stained red) and detrital quartz fragments. B: 12542. Perthite with two stages of growth: plagioclase inner core, with mixed plagioclase/orthoclase outer rim. C: 12542: Perthite fragment with mixed plagioclase and orthoclase. D: 3578-1. Shale lithic fragment in small (<.05 mm) quartz fragments. E: 3578-1: Metamorphic fragment of quartzite (?) surrounded by quartz grains. F: 3578-1. Compacted and sutured quartz grains. Central quartz grain appears to have second episode of quartz growth.

Figure 53. Collected from cuttings. A: 442-1. Shale with layers of fine-medium grain quartz that appear to coarsen up or down (up direction unknown). B: 442-2. Detrital quartz fragments with shell fragments. C: 108. Quartz (?) fragment in fine grained shale. D: 619. Calcite (?) veins in fine grained shale. E: 3137. Volcaniclastic quartz feldspars, and micas, as well as opaques. F: 3137 . Calcite cement.

Figure 54. Electronic log from the type well 788. Clastic and basement tops are picked. Above the clastic surface is a transition to the limestone/dolomite interval of the Wood River.

Figure 55. Map of the top of clastic surface in the SFB. The depth of the surface increases toward the south.

Figure 56. Isochore map calculated for the basal clastic succession. Clastic succession thicknens toward the north.

Figure 57. V-shale map calculated for the lower clastic succession in the SFB. Clastic v-shale generally increases to the south.

Figure 58. Terrane map of North America. Modified from Dickinson and Gehrels (2009). 
Figure 59. Potential Tectonic Peri-Gondwanan Terranes along the eastern margin of Laurentia. From Bowman and Weislogel, (2015).

Figure 60. Potential source map for the SGR and SFB of Florida. The SGR is marked above. The SFB rests above the South Florida Extrusive volcanics. Modified from Dallmeyer, (1987) Heatherington et al. (1997); Mueller et al. (2014)

Figure 61. Cumulative plot and interpreted terranes for the SFB.

Figure 62. Percentage of each terrane population present in the SFB. The SFB is dominated by a Gondwanan/Peri-Gondwanan Peak (525-680 Ma).

Figure 63. Percentage of each terrane present in the SGR. The SGR contains a mix of Gondwanan and Laurentian Terranes.

Figure 64. Percentage of each terrane present in the SGR. The SGR contains a mix of Gondwanan and Laurentian Terranes.

Figure 65. Late Carboniferous provenance model for the Santa Rosa Formation in Western Yucatan. Florida and West Africa served as a dominant source of sediment for the Santa Rosa. From Weber et al. (2006). F-Florida, Y-Yucatan, SM-Southern Maya Block, R-Rokelides, Cho-Chortis block, BR-Brasiliano, Chis-Chiapas Massif, CA-Colombian Andes, BA-Bassarides-Mauritanides, Ac-Acatlan Complex, D-

Figure 66. QtFL ternary diagram for the SFB. The majority of the samples are dominated by quartz and feldspar, interpreted as cratonic interior. Perthites are common in select samples. The Wood River was likely derived from Paleozoic cover of the Suwannee Terrane, and the Osceola Granite. Diagram modified from (Dickinson and Suczek, 1979).

Figure 67. Relative proportion of terrane sourcing for each sample from the SGR. 102

Figure 68. Provenance of the Norphlet Formation in the EGOM from previous studies. The Norphlet is dominated by Cratonic Interior, Transitional Crust, and Recycled Orogen sources. 104

Figure 69. CDP for the 8 major groupings identified in this study. Major groupings are present around $530 \mathrm{Ma}$ and $1000 \mathrm{Ma}$.

Figure 70. Composite Normalized PDP for the Mesozoic EGOM. Results from this study, Lovell (2010), and Lisi (2013).

Figure 71. Provenance model for the Norphlet, Haynesville, Wood River, and Newark in the EGOM from this study. Gondwana, Laurentia, and mixed sources are identified. The 8 groupings identified are listed in Figure 71.

Figure 72. Proposed location of the Suwanne suture, based on work from this study, Lovell, (2010), Lisi (2013), Jessee and Weislogel, (2014) and Heatherington and Mueller et al. (2014).

Figure 73. Paleo-transport model for Laurentia during the Triassic Jurassic. The Appalachian shed sediment that was transported to the Gulf of Mexico, and to the Jurassic ergs systems of the western US. Appalachian sediment was also transported to the Inner Cretaceous Seaway, and southern Canada. Peri-Gondwanan terranes were also a major source of sediment into the western EGOM and the Jurassic 
erg systems. Sediment from Yucatan sourced the San Cayetano Formation, and possibly the South Florida Basin. Modified from Weislogel et al. (2015). Navajo zircon work from Dickinson and Gehrels, (2009), San Cayetano from Rojas-Agramonte (2008).

Figure 74. Propsed chronostratigraphic Correlation between the SGR, EGOM, and SFB. Correlation is based on Formation ages and detrital zircon fresults. SGR ages from Schlische et al. (2003); Chowns and Williams. (1983). EGOM ages from Moy and Traverse, (1986); Goldhammer, (2001); Mancini et al.

(1985); Hammes et al. (2001); Cohen et al. (2013). SFB ages from Pollastro et al. (2001); Heatherington and Mueller, (2003); Cohen et al. (2013). Sea floor spreading from Hudec et al. (2013). CAMP ages from Hames et al. (2000). 


\section{LIST OF TABLES}

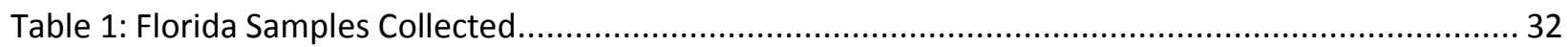

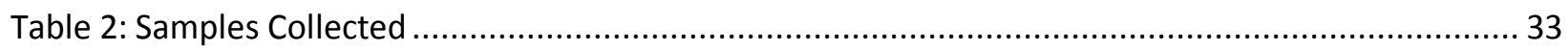

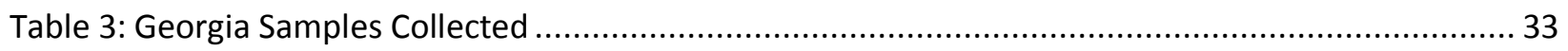

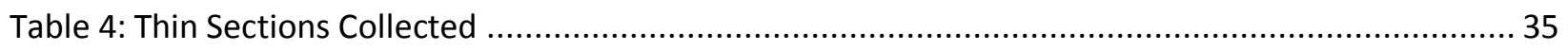

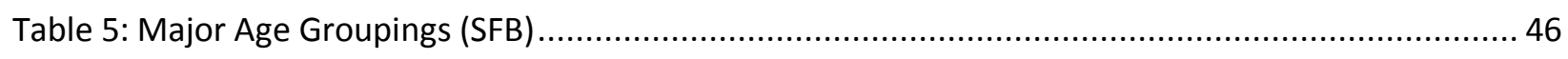

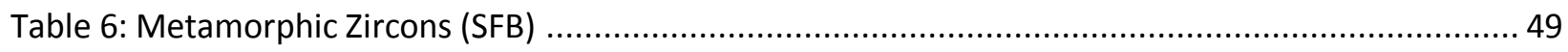

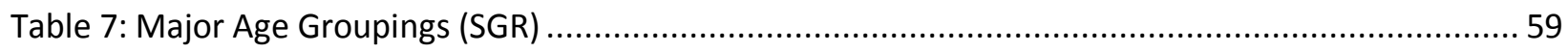

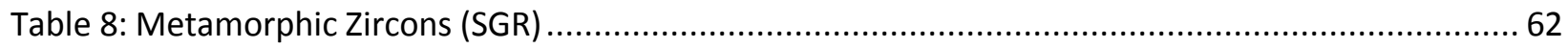

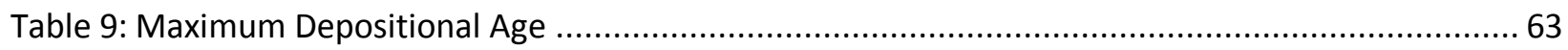

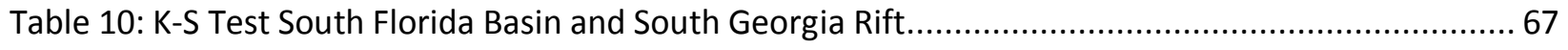

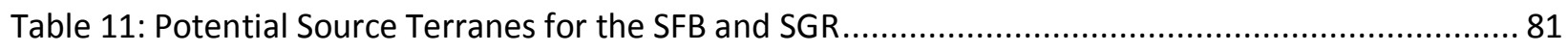

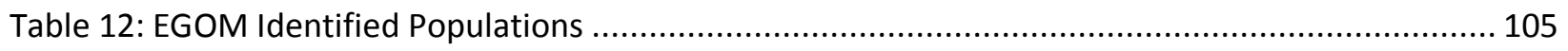

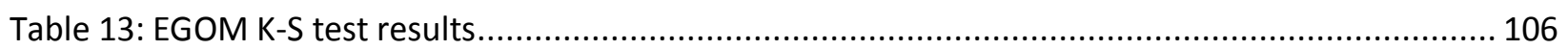




\section{Chapter 1: Introduction}

\section{Introduction of Research Area}

Mesozoic rocks within the eastern Gulf of Mexico (EGOM) have received considerable attention since the 1960's for hydrocarbon exploration (Mancini et al., 1985). Offshore drilling in federal waters of the EGOM has been fairly limited and is currently under moratorium due to the GOM Energy Security Act (2006), which will be in effect until at least 2022; however drilling still continues in state waters of southern Alabama, and on land in the Florida panhandle, and southern Florida (BOEM, 2015). Recent discoveries in the Jurassic Norphlet Formation and Smackover Formation offshore of Alabama and the potential for future discoveries in the offshore western Florida has fueled interest in exploration of these resources (e.g, Rojas, 2015). The extent of the Jurassic Norphlet Formation in southern Alabama/northern offshore Florida has been fairly well delineated, although its regional extent into southern offshore Florida is debated (Mancini et al., 1990; Hunt, 2012). Hydrocarbon discoveries in other Mesozoic units including the Smackover, Haynesville and Cotton Valley formations (e.g, Presley and Reed, 1984; Mancini et al., 2004; Hammes et al., 2011) have spurred interest in the region; however other units such as the Eagle Mills, the basal Wood River Formation of the South Florida Basin (SFB; Fig. 1) and Newark Supergroup of the South Georgia Rift (SGR; Fig. 1) remain relatively unexplored.

Along the Gulf of Mexico margin, a series of Triassic grabens lie buried underneath younger Cenozoic coastal plain deposits. These early Mesozoic units can provide insight into early plate kinematics of EGOM opening and potential source terranes available to supply sediment into the EGOM. In addition, early Mesozoic rift basins that developed during the breakup of Pangea may have provided sediment sources as well as pathways for sediment influx 
to the Norphlet Formation eolian erg and other Jurassic units of the Florida panhandle and offshore DeSoto Canyon (MacRae and Watkins, 1995; Weislogel et al., 2015).

In the EGOM region, these early Mesozoic syn-rift sediments are buried, but scattered wells penetrate the syn-rift section in the South Georiga rift and associated Talahassee graben and in the South Florida basin. The South Georgia Rift (SGR) system formed as a failed rift during the opening of the Atlantic, along the ancient collisional zone of the Suwannee suture (Mueller et al., 2014). From observations of seismic profiles, the SGR consists of several halfgrabens bound by curved listric faults (MacRae and Watkins, 1993; Akintunde et al., 2013; Clendenin, 2013; Heffner, 2013). Wells into the half-grabens penetrate thick packages of Middle Triassic-Lower Jurassic (?) fluvial and lacustrine syn-rift redbeds referred to as the Newark Supergroup (e.g., Chowns and Williams, 1983; Schlische et al., 2003). These units are considered similar in age and lithology to the Newark Supergroup deposits preserved in other extensional basins along the eastern margin of the U.S (Chowns and Williams, 1983; Rine, 2014). The Tallahassee graben is located in northwest Florida and is likely contiguous with the SGR, and SGR-related extensional basins may extend into the Apalachicola Embayment (MacRae and Watkins, 1993; Hunter, 2014). Previous drilling in the SGR have detected minor gas and oil shows, but no commercial drilling has taken place (Coleman et al., 2015).

Along the western margin of Florida, a series of paleotopographic highs, including the Middle Ground Arch and Sarasota Arch, and lows, such as the Apalachiacola embayment (Hunter, 2014) and Tampa embayment (Wilson, 2012), have been imaged using seismic reflection data, however the origin of these is controversial. The southernmost of these paleotopographic lows is the South Florida basin (SFB; Gregg, 2014). The SFB is interpreted as a continental rift basin that formed during the initial breakup of Pangea (Pollastro et al., 2001); 
however, it mainly contains thick carbonate sequences deposited throughout the Cenozoic and Cretaceous, indicating Florida was a stable, carbonate platform during much of its history.

At the base of these carbonates, a thin unit of Upper Triassic/Jurrasic (?) redbed sandstone and shale known as the Wood River Formation rests above basement and Triassic volcanic rocks. A horizion identified through seismic imaging of the offshore West Florida Basin may also contain basal sands that may be similar in age (Fig. 1., Gregg, 2014); these also lie beneath thick carbonate sequences (Applegate et al., 1981). The basal section of the Wood River Formation likely records early syn-rift sedimentation in the SFB. 


\section{Sample Locations}

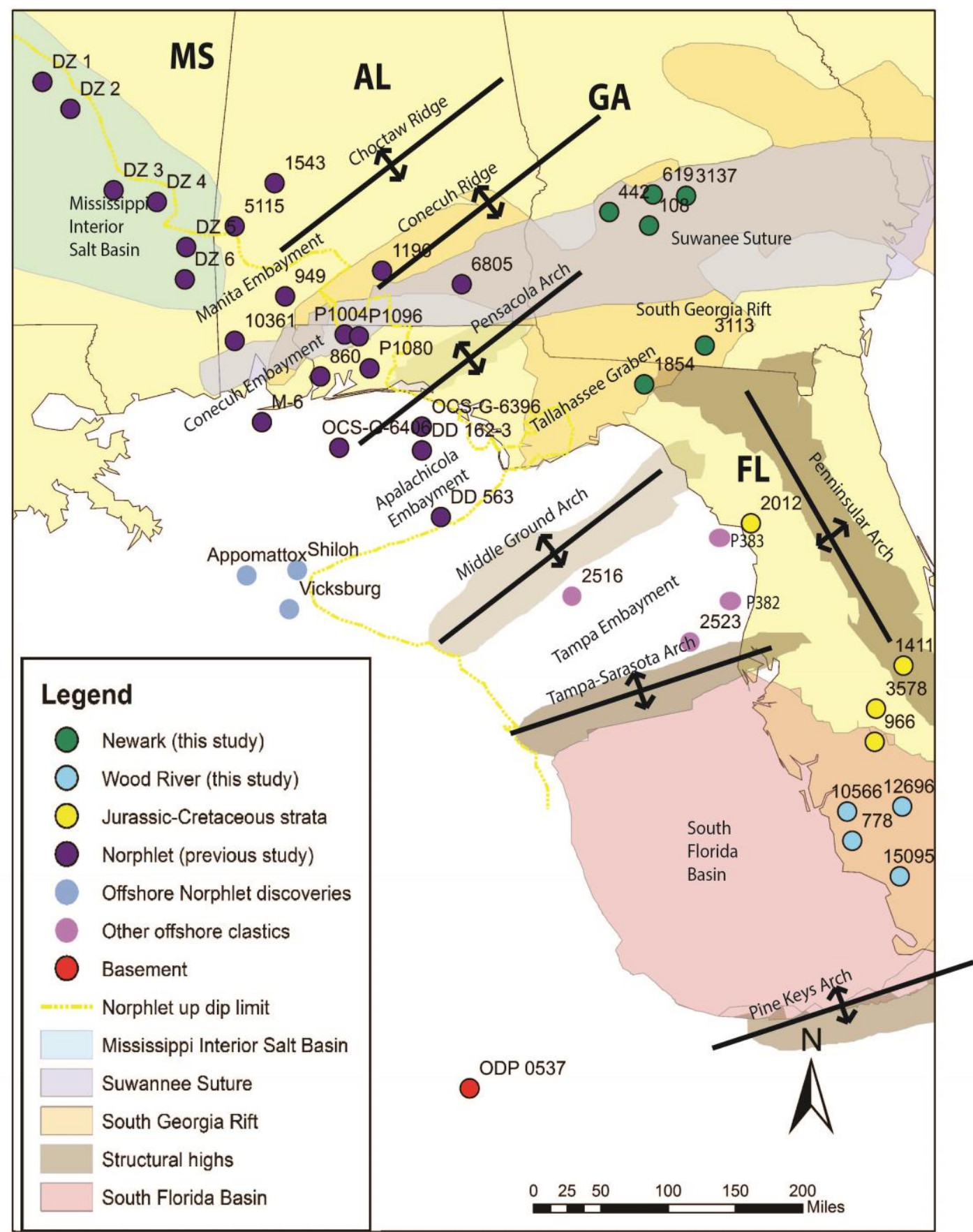

Figure 1. Location of the SFB and SGR in the EGOM. Local structural highs and lows are also identified. Samples collected for this study are listed as Basement, Jurassic-Cretaceous, and Wood River. Previous samples collected from the Norphlet and other offshore clastics are also displayed from Lovell (2010) and Lisi (2013). Structures from Neathery and Thomas (1975); Christenson, (1990); Pollastro et al. (2001). Location of the SGR from Chowns and Williams (1983); Suwannee Suture from Mueller et al. (2014). 


\section{Project Goals}

Identifying the provenance of Triassic/Jurassic clastic rocks in the SGR, Tallahassee

Graben and SFB can identify sediment source terranes available to supply sediment to the EGOM basin during initial rifting, and could test potential sediment transport pathways hypothesized for the Norphlet in the EGOM (e.g., Lovell and Weislogel, 2010; Weislogel et al.,

Middle Jurassic (175-155 Ma)

Continental crust

Continental shelf/Transitional crust

Marine evaporites

Non marine/Shallow marine

clastics

$\overbrace{\text { North }}^{N}$

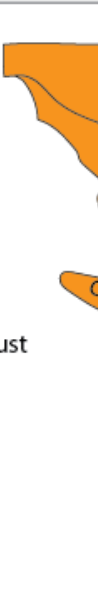

2015). This thesis reports provenance analysis of

Triassic/Jurassic basal clastic rocks in the SGR and SFB using detrital zircon $\mathrm{U}-\mathrm{Pb}$ geochronology and other methods in order to
Figure 2. Reconstruction of the Gulf of Mexico, with initial separation of Yucatan from Laurentia, and early initiation of the Caribbean during the Middle Jurassic (170 Ma). Modified from Iturralde-Vinet (2003) and Hine (2013). identify the origin of sediment influx into the early EGOM. Zircon grains are proven to be

excellent provenance tools, due to their ability to be transported long distances without significant physical or chemical weathering (Gehrels, 2014). Although zircon typically crystallizes from felsic magmas, they can also crystallize from mafic magmas if zircon saturation is attained (e.g, Hoskin and Schaltegger, 2003). Zircons contain little initial Pb, so Pb levels measured in zircon are modeled as the result of decay from $\mathrm{U}$ to $\mathrm{Pb}$, which permits calculation of a radiometric crystallization age. Comparing the ages of detrital zircons with age of zircon from 
igneous or metamorphic rocks of potential source terranes allows for provenance determination and reconstruction of sediment transport pathways.

The Triassic-early Jurassic arrangement of microplates and their relationship to larger plates in the EGOM region during early Mesozoic evolution is poorly constrained, and a variety of tectonic terranes, including Laurentia and associated Precambrian-Paleozoic orogenic belts, South America, West Africa, and small microplates such as Yucatan, may have shed sediment into the SFB (Fig. 2). Zircon age signatures of these potential source terranes vary due to their disparate tectonic evolution. Therefore, comparison of source area to the zircon age signatures of basal deposits of the Jurassic Wood River Formation, and Triassic syn-rift deposits can be used to constrain the distribution of terrane(s) that served as sediment sources. Thin-section point counting supplements $\mathrm{U}-\mathrm{Pb}$ detrital zircon provenance analysis to identify tectonic setting of the source area (Dickinson and Suczek, 1979). Together, detrital zircon ages and sandstone modal composition data can be used to characterize sediment provenance of lower Mesozoic units in the EGOM in order to test and expand previous Late Jurassic EGOM paleo-geographic models, and refine inferred sediment pathways.

\section{Research Problem Significance}

Results of this project will address: 1) the paleogeography of the Early EGOM, 2) provide information regarding the distribution of Jurassic sedimentary reservoirs in the EGOM, 3) help to delineate the Suwannee suture, a major tectonic boundary in the EGOM region, and 3) constrain maximum depositional age of basal units in the EGOM. Reconstructing the origin and distribution of early Mesozoic EGOM deposits is problematic due to the significant tectonic, paleogeographic reorganization and burial that has taken place during subsequent late Mesozoic and Cenozoic evolution of the GOM. Until the Early Cretaceous, drainage systems into Gulf of 
Mexico were primarily transporting sediment derived from Appalachian sources. By the Paleocene, tributary systems feeding the Gulf of Mexico extended throughout the American southwest and northwest of North America and Canada (Galloway et al., 2011; Blum and Pecha, 2014). Provenance signatures among lower Mesozoic EGOM units can be used to assess paleodrainage catchment configuration and potential connections between onshore sources and offshore lower Mesozoic deposits.

Ultimately, comparing provenance of Triassic-Jurassic syrift deposits with provenance of the Norphlet Formation erg in the northern EGOM as determined through previous work will provide constraints on potential for lower Mesozoic reservoir units across the EGOM (Mancini et al., 1985; Lovell, 2010; Hunt, 2012; Lisi, 2013; Weislogel et al., 2015). Previous exploration success with the Norphlet deep-water play in the EGOM supports the potential for future offshore discoveries. Early exploration attempts in the Norphlet Formation around the offshore DeSoto Canyon lease block were largely unsuccessful, largely due to low porosity (Godo, 2006). The first major discovery was made by Shell at the Appomattox well in 2010, with 550 mmboe (Shell Fact Sheet, 2014). Shell discoveries in the Mississippi Canyon Block include the Appomattox, Vicksburg, Rydberg, and most recently the Fort Sumter (World Oil, 2016), indicating the full extent of the play has not been delineated.

The potential for reservoirs is currently untested, but drainage configuration hypothesized by Weislogel et al., (2015) predicts a sediment feeder system routed through the Tallahassee Graben and/or Tampa Embayment into the EGOM. The SGR and Tallahassee Graben have been interpreted to extend into the EGOM offshore near the Apalachicola Embayment and the DeSoto Canyon area (McRae and Watkins, 1996), and Wilson (2012) identified possible Norphletequivalent deposits in the Tampa embayment; these sediments may have originated from a 
transport system in the Tallahassee Graben (Figure 1). The extent of the Norphlet reservoir facies south of the Sarasota Arch is not well constrained, but seismic analysis by Gregg (2014) identified possible Norphlet equivalent deposits in the West Florida Basin. Establishing input through Florida rift systems would increase the probability for presence of reservoir units in this part of the EGOM.

The exact trace of the Suwannee suture which connects Florida (Gondwana) to North America (Laurentia) is currently unknown (Mueller et al., 2014). The Suwannee Suture has been interpreted to parallel the SGR and at least partially coincide with the Brunswick Magmatic Anomaly (BME), a magnetic low that parallels the SGR and extends into the offshore Atlantic and southern Alabama (Chowns and Williams; 1983; Williams and Hatcher, 1983; Hatcher et al., 2010). However, wells north of the BME tag basement that resembles rocks of the Suwannee terrane, which may indicate the BME is a result of overthrusting of Suwanee-related rocks onto Paleozoic rocks of the Laurentian lower plate (Hatcher et al., 2010), or it may reflect periGondwanan terranes that had accreted to Laurentia prior to Alleghenian orogenesis (e.g., Horton et al., 1989; McBride et al., 2005; Hatcher, 2010). Since the Suwanee suture separates Gondwanan from peri-Gondwanand and Appalachian orogenic rocks, zircon ages of syn-rift deposits can be used to constrain the location of the Suwannee suture, with Laurentia-derived zircons expected near the Laurentia side of the suture, and Gondwana influenced sources near the Suwannee side of the suture. This will permit an assessment of the influence the suture zone exerted on structural and sedimentation patterns produced from Triassic rifting. Results from this project will test previous tectonic models for the configuration of the Suwannee suture identifying variations in detrital zircon signatures across the basin. 


\section{Chapter 2: Background}

\section{Tectonic Setting and Evolution of the Mesozoic EGOM}
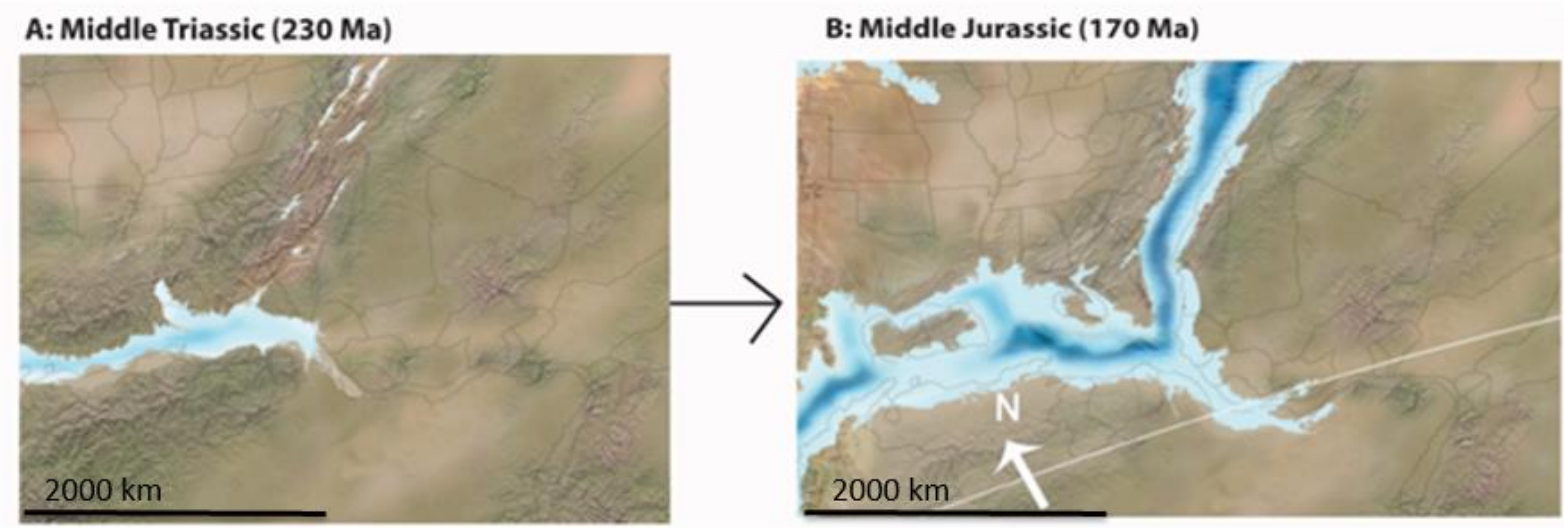

Figure 3. Paleogeography in the Gulf of Mexico from the Triassic (230 Ma) and the Jurassic $(170 \mathrm{Ma})$. Modified from Blakey (2016).

The Gulf of Mexico is generally interpreted as a rift basin that formed during the breakup of Pangea starting sometime around 204 Ma (Fig. 3; Dobson and Buffler, 1997). An alternative hypothesis by Stern and Dickinson (2010) suggests that the GOM formed from as a backarc basin related to eastward subduction in western South America. Rifting of Pangea occurred along zones of continental weakness, including sutures where previous terranes had accreted and ancient rifts from the breakup of Rodinia (Salvador, 1987; Hatcher, 2010; Mueller et al., 2014). Tholeiitic basalts found in the Tallahassee graben of northern Florida/South Georgia Rift basins initially formed during opening of the Atlantic ocean as part of the Central Atlantic Magmatic Province (CAMP; e.g, Austin, 1990; Hames et al., 2000; Fillon, 2007), which includes volcanism throughout eastern North America, western Africa, and eastern South American (Austin et al., 1990; Marzoli et al., 1999; Heatherington and Mueller, 2003). Basalt ${ }^{40} \mathrm{Ar} /{ }^{39} \mathrm{Ar}$ ages around 200 
Ma indicate the age of the main phase of rifting in the EGOM (Hames et al., 2000). Basalt and rhyolite in the South Florida Basin and offshore western Florida range in age from 175 to 199 Ma, consistent with a Central Atlantic Magmatic Province (CAMP) association, and may indicate another phase of younger volcanism (Dallmeyer, 1983; Heatherington and Mueller, 2003). Extension along the eastern margin of the developing North American continent created a series of basins that infilled with fluvial and lacustrine strata of the Newark Supergroup (e.g., Chowns and Williams, 1983; Smoot, 1991). Many of these basins from Nova Scotia to North Carolina are exposed at the surface (Fig. 5; Withjack et al., 1998); however, the basins continue southward in the subsurface and include the South Georgia Rift (SGR) basin system.

The SGR is a buried Triassic-Jurassic graben rift basin system along the southeastern Atlantic margin of the U.S. that has been infilled with Mesozoic sediment and volcanic rocks (Chowns and Williams, 1983). The Newark Supergroup rocks are proven to include hydrocarbon source rocks, although previous exploration attempts have not proved commercial (Milici et al., 2011; Coleman et al., 2015). The general orientation of SGR sub-basins is NE-SW relative to the N-S orientation of other rift basins along the U.S. Atlantic margin, likely due to structural weaknesses of the NE-SW oriented Suwannee suture (Chowns and Williams, 1983; Klitgord et al., 1988; Mueller et al., 2014). Rifting along the SGR axis failed and continued to the east until seafloor spreading intiated. The region remained a topographic depression, and during periods of sea level rise in the Cretaceous and Cenozoic, the SGR area was submerged by seawater, forming the Georgia Seaway (also known as the South Georgia Trough, or Georgia Channel System; Warzeski et al., 1996; Hine, 2009). The SGR was originally proposed as a series of separate rift basins within a single larger rift basin by Daniels et al. (1983), which is supported by further seismic and aeromagnetic studies (e.g., Kiltgord et al., 1988; Sartin and See, 
1997; Heffner, 2013). In his study of the SGR structural development, Heffner (2013) identified several sub-basins separated by transfer faults within the SGR, including the Albany Basin and the Valdosta Basin, as well as the Riddleville/Dunbarton Basin and Kibbee Basin (Fig. 4). Of these, the Albany Basin contains significantly thicker strata (upwards of $~ 3990$ meters thick) than other sub-basins, based on seismic interpretation (Heffner, 2013). Maximum basin fill for the SGR approaches 4270 meters in Georgia (Satin and See, 1997).

Evidence of basin inversion is present in many of the rift basins of the eastern U.S., and

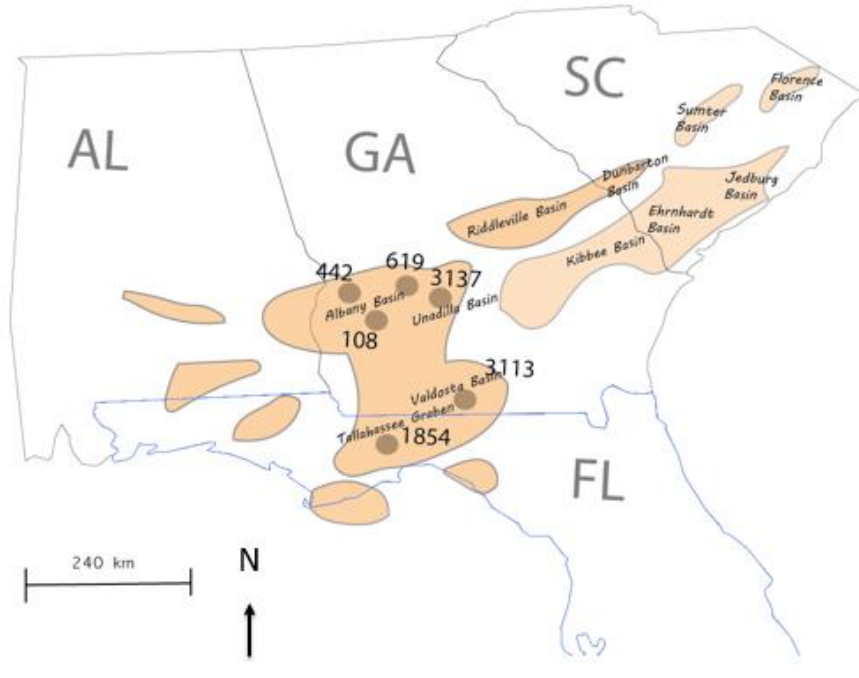

Figure 4. Location of the various SGR sub-basins, modified by Heffner (2013). SGR sample locations from this study are shown. may have occurred in the SGR (Withjack

et al., 1998; Schlische and Withjack, 2003)

Rine et al. (2014) reconstructed the burial history of the Newark Supergroup in South Carolina from core. A maximum burial depth of $\sim 3500$ meters was attained for the interval sampled (2600-6200 ft), followed by deposition of Jurassic strata. Subsequent basin inversion during the Mesozoic around 200 Ma resulted in at least 2800 meters of uplift based on $\delta^{18} \mathrm{O}$ of calcite cement (Rine et al., 2014). Inversion may have resulted in significant erosion of strata from exposed rift basins. Uplift ended sometime before deposition of the Cenozoic cover strata (Schlische and Withjack, 2002; Rine et al., 2014).

Along the northern margin of the Gulf of Mexico, a similar series of NE-SW oriented rift basins separated by paleo-highs of thicker, less attenuated crust developed during early Mesozoic 
time as the Yucatan Block separated from North America and migrated southward, (Fig. 6; MacRae and Watkins, 1993; Dobson and Buffler, 1997; Wilson, 2011). Paleo-highs in the vicinity of Florida include the Pensacola Arch, South Platform/Middle Ground Arch, and Sarasota Arch, and the intervening paleo-lows include the Apalachicola Embayment, DeSoto Canyon Salt Basin, Tampa Embayment, and South Florida Basin (Dobson and Buffler, 1997; Wilson, 2012). These structures parallel the ancient collisional structures of the Alleghany orogen, so the rift structure orientations may be related to lithosphere heterogeneity inherited from earlier collision events.

During the initial phase of rifting, the Yucatan Block rifted off southern Laurentia and begun to rotate into its current position off South America (Pindell and Kennan, 2009). Previous workers had speculated that another continental block, the Florida-Bahamas Block, rifted off the southern margin of Laurentia and migrated toward the southeast along transform faults along the western margin of the Suwannee Terrane, before attaching to the southern end of the terrane, resulting in a series of tilted fault block basins in southern Florida (Klitgord et al., 1988). However, the existence of the Florida-Bahama block remains speculative, and recent seismic data show no evidence for transform faulting along the western margin of the Suwannee terrane (Wilson, 2011). A seafloor spreading center developed in the Gulf of Mexico that further separated Laurentia from South America beginning in the Middle Jurassic (Fig. 5; Salvador, 1987; Hudec et al., 2013; Boschman et al., 2014). As sea level rose through the Cretaceous and Cenozoic, carbonate platforms accumulated to form the Florida escarpment whereas the northern EGOM coast accumulated a mix of carbonate and clastic deposits (Mancini et al., 1985; Pollastro et al., 2001). 

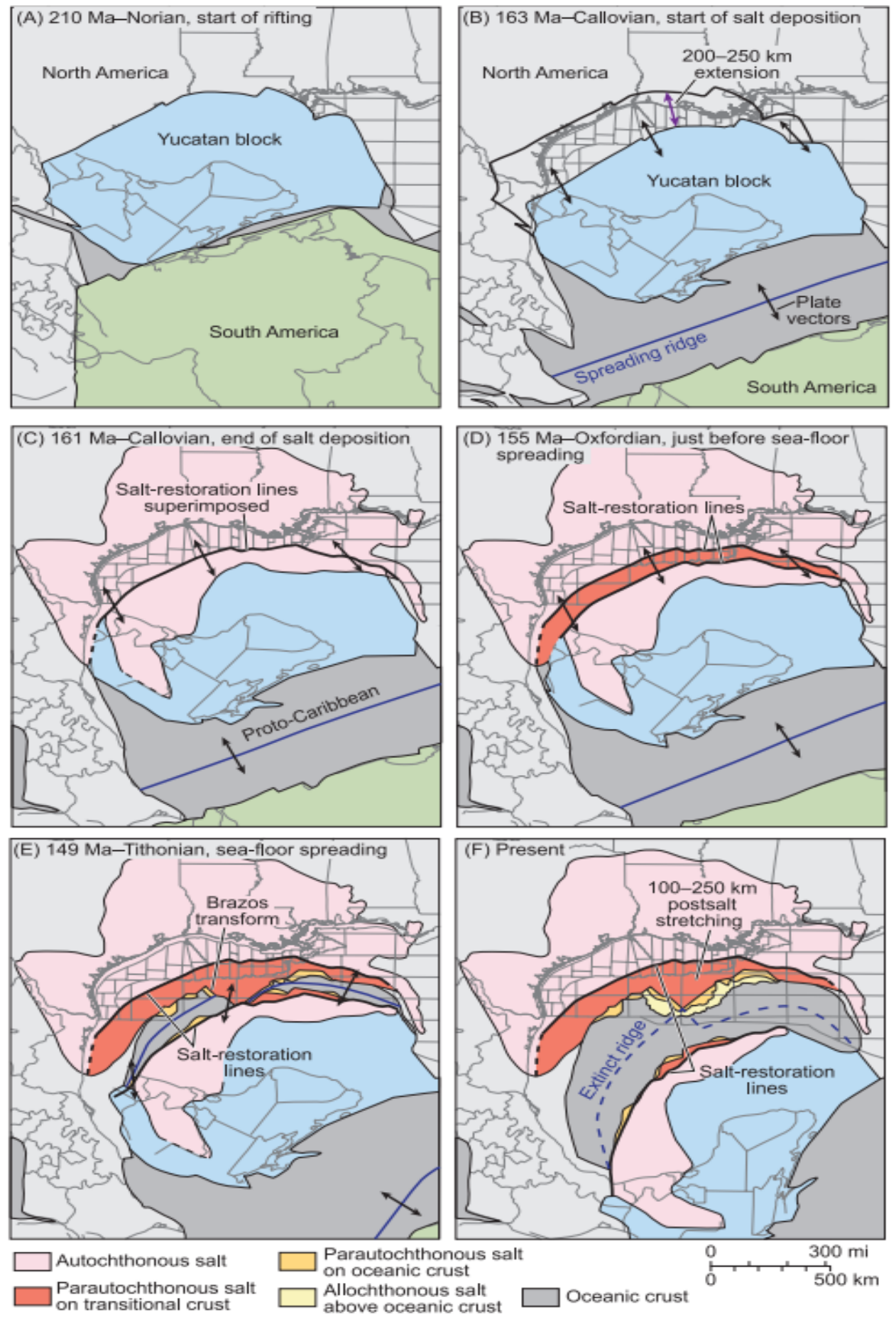

Figure 5. Model for rifting and seafloor spreading and rifting from Hudec et al. (2013). A: $210 \mathrm{Ma}$, initial rifting of Yucatan from Laurentia. B: $163 \mathrm{Ma}$, initial deposition of Louann salt in the GOM following rifting. C: End of salt deposition south of Texas. D: $155 \mathrm{Ma}$, continued extension of crust and salt deposits, E: $149 \mathrm{Ma}$, seafloor spreading begins. F: Modern extent of the GOM, salt, and ocean crust. 


\section{Basement rocks: Paleozoic orogens and Gondwanan Suwannee Terrane}

Basement rocks along the eastern margin of Laurentia consist of accreted terranes and synorogenic rocks that developed from Proterozoic to Paleozoic time. The Grenville orogen (900-1300 Ma) extends from the eastern margin of Canada and North America, and formed during the initial assembly of Rodinia (Bream et al., 2004; Rivers, 2008; McLelland et al., 2010). Throughout the Paleozoic, a series of tectonic terranes accreted onto the eastern margin of Laurentia, known as the Appalachian orogenic events. These include the Taconic (430-490 Ma), Acadian/Neoacadian (330-420 Ma), and Alleghanian (265-330 Ma) orogenic events (Hatcher, 1987; Miller et al., 2000; Moecher et al., 2004; Sinha et al., 2012; Steltenpohl et al., 2013). A series of Peri-Gondwanan Terranes, including the Charleston, Savannah River, Milledgeville, Uchee, and Carolina Terranes accreted onto Laurentia through the Paleozoic (525-680 Ma), and now forms the basement of most of eastern South Carolina and eastern Georgia (Wortman et al., 2000; Coler et al., 2000; Hatcher, 2010; Mueller et al., 2014). Peri-Gondwanan Terrane accretion culminated with the collision of Gondwana with Laurentia during the Alleghanian orogeny; a remnant of Gondwana known as the Suwannee terrane remains attached to North America along the Suwannee Suture and comprises the basement of modern-day Florida (Mueller et al., 2014).

After accreting onto North America, some models postulate the northern portion of the Suwannee Terrane was translocated into its current position by transform movement along the Suwannee Suture during opening of the GOM (Mueller et al., 2014). The exact location of the Suwanee Suture in the subsurface is unknown, and has been the subject of numerous studies (e.g., Mueller et al., 2014; Parker, 2014); however, currently it is thought to be stretch from offshore South Carolina across southern Georgia and into southern Alabama, at least partially coincidental with the Brunswick Magnetic Anomaly and/or the trend of the SGR (Chowns and 
Williams, 1983; Williams and Hatcher, 1983; Hatcher et al., 2010; Steltenpohl et al., 2013).

Current seismic refraction studies in southern Georgia will better constrain the exact location of the Suwannee suture (e.g., Shillington et al., 2014).

Suwannee terrane basement includes igneous, metamorphic and sedimentary provinces. Igneous and metamorphic provinces associated with the Suwannee Terrane consists of the Osceola Granite (552 Ma), the St. Lucie Metamorphic Complex (530 Ma), and rift related North Florida Volcanics ( 199 Ma) and South Florida Volcanics (175 - 199 Ma; Dallmeyer et al., 1987; Dallmeyer et al., 1989; Mueller et al., 1994; Heatherington and Mueller, 1997; Heatherington and Mueller, 2003). Paleozoic sedimentary rocks found throughout the Florida subsurface range in age from Ordovician to Devonian (Applin, 1951; Chowns and Williams, 1983) and yield 525-680 Ma and 2,000-2,250 Ma detrital zircon ages, which is consistent with Pan-African Brasiliano sources and Eburnean/Trans-Amazonian orogenies (Mueller et al., 1994; Mueller et al., 2014). The lack of any zircon with ages corresponding to Laurentian sources suggests that the pre-Mesozoic Suwannee Terrane consists of African and South American sedimentary cover over Pan-African basement (Mueller et al., 2014). A possible correlation between Suwannee Paleozoic sedimentary cover has been proposed to sandstones with similar lithologies to the Bove Basin in West Africa, however recent detrital zircon data from the Bove basin have a prominent 1200-1300 Ma signature that is absent from the Suwannee Basin (Dallmeyer et al., 1987; Mueller et al., 2016). 


\section{Generalized Mesozoic Stratigraphy of the Northeastern Gulf of Mexico}

The Triassic Eagle Mills Formation sits unconformably on Paleozoic basement and formed during initial syn-rift clastic sedimentation in the EGOM (Fig 6; Mancini et al., 1985).

Although the

formation is poorly

Eastern Gulf Of Mexico Stratigraphty

studied, a Carnian

(Late Triassic) age for

the Eagle Mills

Formation is assigned

based on

palynomorphs, (Moy

and Traverse, 1986).

During early

extension and seafloor

spreading, the GOM

experienced

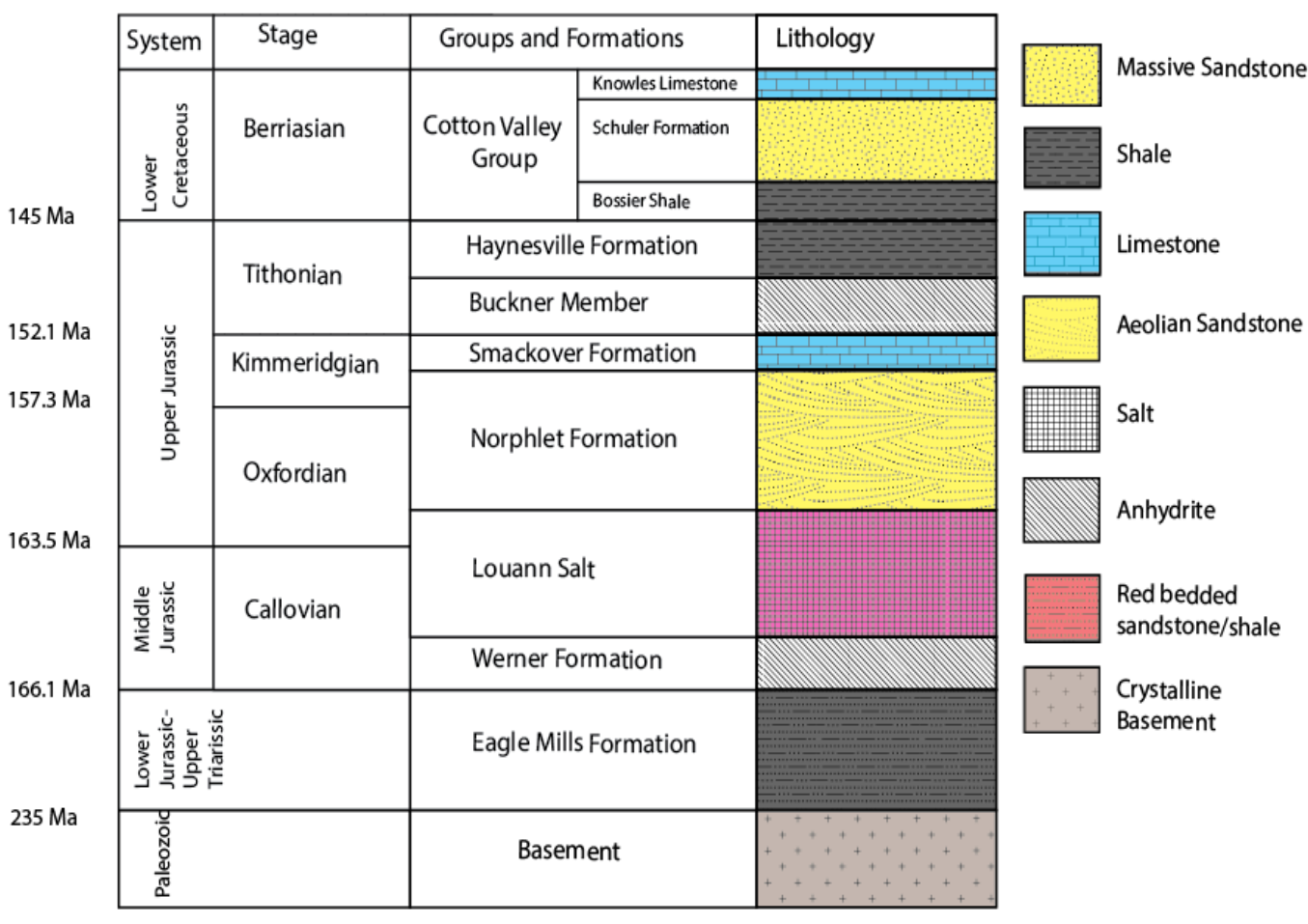

occasional flooding by

Figure 6. Stratigraphy of the EGOM. Modified from Mancini et al. (1985). Ages from Goldhammer et al., (2001); Cohen et al. (2013).

seawater; periodic

evaporation resulted in the deposition of the overlying Werner Formation and Louann Salt (Fig.

7; Dobson and Buffler, 1997; Hudec et al., 2013). Deposition of Louann Salt began in the

Callovian (163 Ma), and continued throughout continental rifting until seafloor spreading began

around $155 \mathrm{Ma}$ (Hudec et al., 2013). Thick deposits of the Louann Salt accumulated across much

of the early GOM, including the central onshore and offshore Gulf of Mexico and select regions in eastern Mexico. 
Greater evaporate accumulation occurred in areas of higher crustal attenuation and subsidence (Tew et al., 1991; Dobson and Buffler, 1997). Thickest accumulations of lower Jurassic evaporites in the EGOM formed in the Mississippi Interior salt basin; these evaporites thin southeastward toward the Apalachicola basin until they pinch out in the southern EGOM, and so are completely absent from the South Florida Basin and West Florida Basin (Applegate et al., 1981; Wilson, 2011; Hudec et al., 2013; Gregg, 2014). Hudec et al. (2013) hypothesized that salt deposition was restricted to zones of crust that had been hyperextended or that had evolved into transitional crust, indicating that seafloor spreading in the southern EGOM pre-dated salt deposition. As the basin began to subside following rifting, a thick sequence of JurassicCretaceous post-rift sediment was deposited on top of the Louann Salt, beginning with the Upper Jurassic Norphlet (Dobson and Buffler, 1997).

The Upper Jurassic Norphlet Formation clastic deposits formed in an arid environment by alluvial fan, wadi, eolian, and fluvial systems (Mancini et al., 1985). In Alabama, where the Norphlet Formation is best characterized, alluvial fan conglomerate facies were deposited immediately adjacent to highlands of the remnant Alleghany orogen, and wadi and fluvial systems deposited red bed sandstone and shale in the more distal regions (Mancini et al., 1985; Dobson and Buffler, 1997; Hunt, 2012). Further from the Alleghany orogen, sediment was deposited in an eolian erg, which includes dune, interdune/wadi, and playa lake environments that formed well sorted, quartz-rich, thin- to thick-bedded sandstones (Mancini et al., 1985; Gutierrez and Ewing, 2015). The upper section of the Norphlet in southern and offshore Alabama is composed of a quartz-rich, well sorted sandstone known as the Denkman Member, described by Mancini et al. (1984). Sedimentary structures in the Denkman Member range from massive sandstone to lower cross-bedded sandstone. The lower section grades into a red cross- 
bedded sandstone, which constitutes the dominant Norphlet lithology in the EGOM region. The red beds coarsen upward into cross-bedded eolian sandstone throughout southern Alabama. The Norphlet Formation grades upward into a carbonate-rich sandstone and eventually into the marine carbonate units of the Smackover Formation (Mancini et al., 1990). The Norphlet Formation contact with the overlying Smackover Formation ranges from sharp to gradational. Lower Smackover Formation facies that is gradational with the Norphlet is known as the Brown Dense, and consists of organic-rich, subtidal mudstone (Claypool and Mancini, 1989). The Brown Dense is interpreted to have sourced hydrocarbons found in Norphlet and other clastic reservoirs in the EGOM.

South Florida Basin Mesozoic Stratigraphy

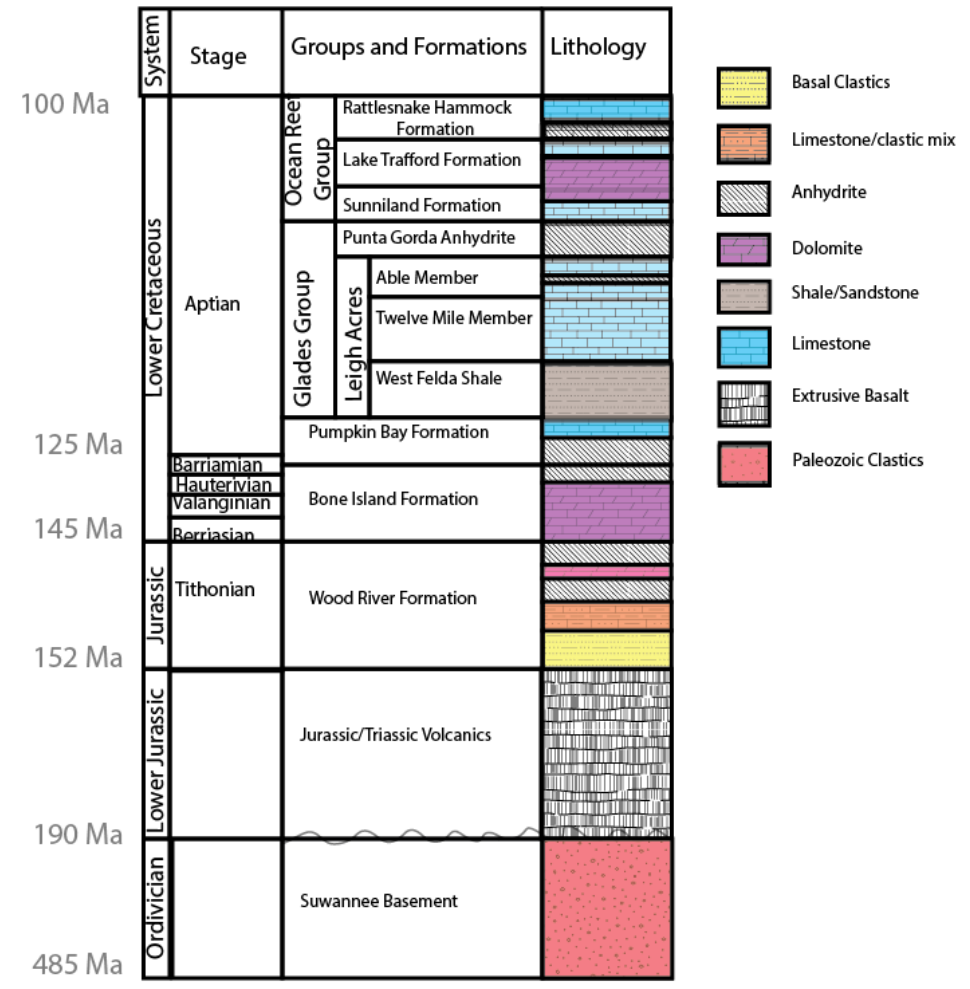

\section{Mesozoic Stratigraphy of the} South Florida basin

Mesozoic rocks of the SFB include Triassic-Jurassic volcanic rocks, thin Triassic-Jurassic basal clastic rocks, and thick Cretaceous carbonate rocks (Fig. 7 and 8; Pollastro et al. 2001). The Wood River Formation forms the basal Figure 7. Stratigraphy of the South Florida Basin. Modified from Pollastro et al. (2001).

sedimentary deposit of the SFB and was first described by Applegate et al. (1981). The Wood River Formation rests below the Bone Island Formation, and above 
Mesozoic volcanic rocks and Paleozoic sedimentary rocks of the Suwannee terrane.

Hydrocarbon exploration and drilling in southern Florida has concentrated mainly on the Lower Cretaceous dolomite and limestone of the Sunniland Formation (Pollastro et al., 2001). Only 3-4 wells have penetrated through the Cretaceous section into the underlying Jurassic Wood River Formation (Applegate et al., 1981), although at least 7 other wells penetrate into basal clastics that are likely related to Wood River Formation deposits. The Wood River Formation in the type well (P-778) contains red, fine- to coarse-grained sandstone and shale, as well as anhydrite and brown microcrystalline dolomite in the upper 36.5 meters (120 feet). Lithologic characterization is based primarily from cutting interpretations and well logs, which reveal the sandstone is composed mainly of quartz and feldspar. Potential depositional environments proposed for the basal clastic Wood River Formation are based on well log signatures, and include marginal marine deltaic, fluvial, and fluvial-lacustrine depositional settings (Pollastro et al., 2001). Although no direct age information exists for the Wood River Formation basal clastic unit, the rhyolite porphyry that underlies the Wood River Formation in the type well has been dated at 189 Ma using Rb-Sr whole rock geochemistry (Applegate et al., 1981; Tapping, 1994) and biostratigraphy from the overlying Bone Island Formation indicate an age of $138 \mathrm{Ma}$ (Applegate et al., 1981); thus, the basal clastic unit of the Wood River was deposited between 138-189 Ma (Pliensbachian-Neocomian). Tapping, 1994 speculated a late Triassic-Early Jurassic age for the Wood River based on the age of similar clastic units in the northern EGOM. Mesozoic volcanic rocks from other wells in the South Florida Basin have been analyzed using $\mathrm{Rb}-\mathrm{Sr}$ and $\mathrm{K}-\mathrm{Ar}$ geochronology, yielding ages between 175 to 199 Ma for basalts, and 165 to $189 \mathrm{Ma}$ for rhyolites (Heatherington and Mueller, 2003). 


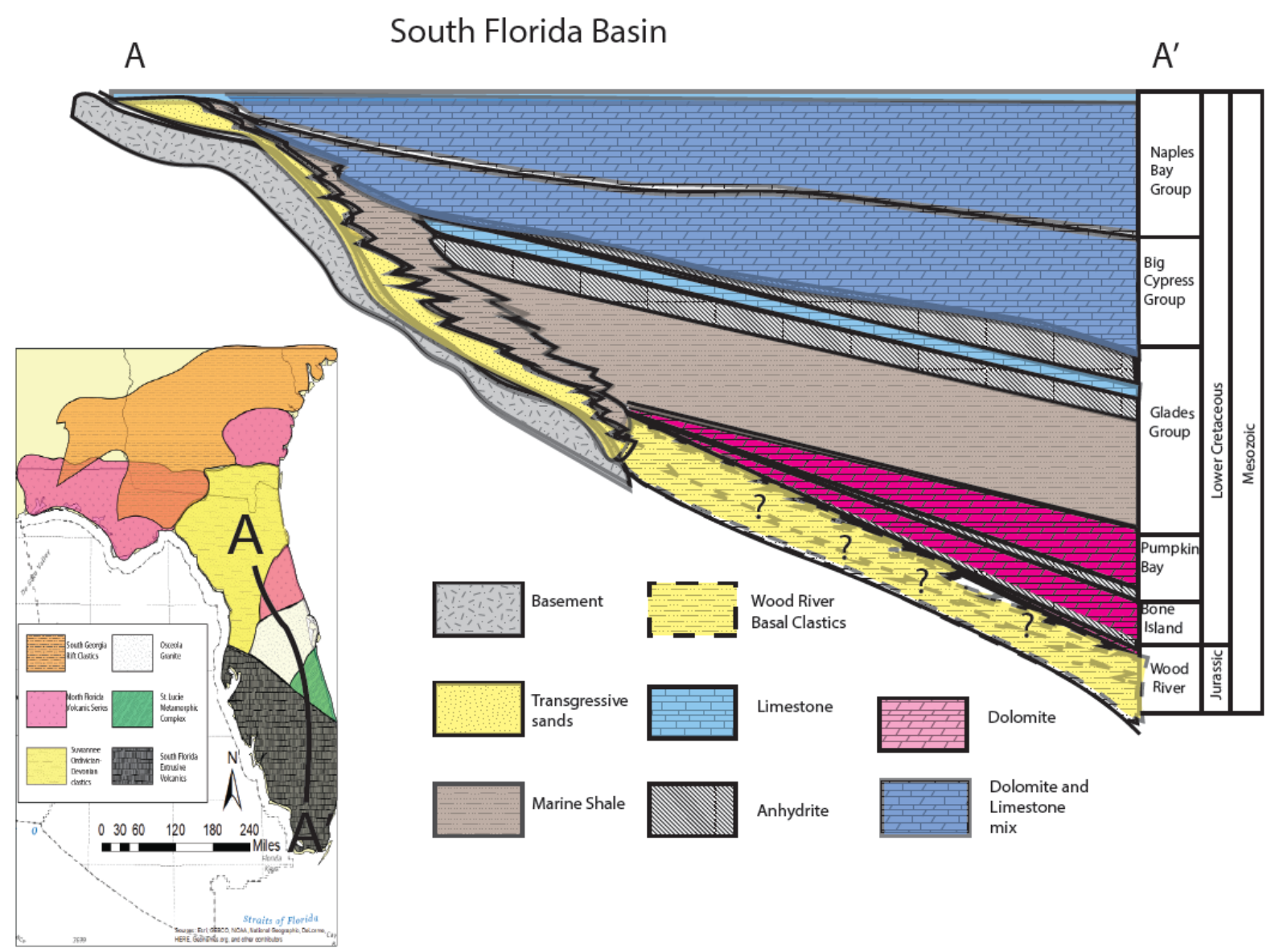

Figure 8. Cross-section of the Lower Cretaceous South Florida Basin. The South Florida Basin is dominated by thick Cenozoic and Cretaceous carbonates. A thin, Triassic/Jurassic clastic layer rests above interpreted basedment. Modified from Pollastro et al. (2001).

\section{Basement and Mesozoic Stratigraphy of the South Georgia Rift}

Syn-rift and post-rift strata in the SGR are referred to as the Upper Triassic (?) Newark Supergroup, which includes sedimentary rocks, felsic and mafic extrusive rocks and diabase intrusions (Chowns and Williams, 1983). Unlike the exposed Triassic rift basins in the eastern U.S, which bear vertebrate and invertebrate fossil assemblages (e.g., Fowell et al., 1994), the age of the SGR basin-fill is poorly constrained due to lack of fossil evidence. Mesozoic strata of the 
SGR consists of at least 3,500 meters of Triassic (?) red bedded sandstone and shale (Chowns and Williams, 1983). Strata generally dip toward the northeast, implying syn-rift deposition during motion along basin-bounding faults forming the northeast basin margin. Core no longer exists for the SGR in Georgia, although some core has been recovered in South Carolina. Core recovered from South Carolina was interpreted to mainly consist of lacustrine and fluvial facies, based on lithofacies and fossils (Rine, 2014). Electrical log data suggests minor fining upward sections and cross-bedding are present. Chowns and Williams (1983) interpreted that the mainly arkosic sandstone (60\%), along with subordinate abundances of red shales and siltstones (40\%) were deposited in a continental fluvial setting, with other alternate possibilities including alluvial fan, eolian dunes, playa lakes, and alluvial fans that fringe the basin margins. Other studies have supported a lacustrine origin (e.g., Ziegler, 1983; Rine, 2014).

The northern SGR rests on and is flanked by basement rocks formed during the Grenville orogeny and Appalachian orogenic events, with main lithologies including granites, schist, and gneiss, and Paleozoic sedimentary rocks (Chowns and Williams, 1983). In the segment of the SGR that likely extends south of the Suwannee suture, the SGR rests on and is flanked by Suwannee terrane rocks composed of quartzose sandstone and red to black shale. Accordingly, two distinct petrofacies are identified for SGR deposits based on composition (Chowns and Williams, 1983). In the northern SGR, sandstones are primarily a mix of arkosic/subarkosic and lithic-arkosic, with a dominance of metamorphic lithic fragments indicating derivation from rocks of the Appalachian metamorphic piedmont (Chowns and Williams, 1983). The southern SGR contains mainly arkosic/subarkosic and litharenites bearing sandstone and volcanic fragments, which are interpreted to have been derived from the Paleozoic sedimentary rocks of the Suwannee Terrane and North Florida Volcanics (Chowns and Williams, 1983). 
Diabase dikes and tholeiitic basalt sills are common in the lower Mesozoic section of the eastern SGR. Clastic rocks surrounding the intrusions exhibit slight contact metamorphism, mainly zeolite and hornfell facies (Chowns and Williams, 1983). Mafic volcanic rocks in the SGR likely related to the Central Atlantic Magmatic Province (CAMP) locally cap the red bed section (Heffner et al., 2012). Isotopic $\mathrm{Ar}^{40} / \mathrm{Ar}^{39}$ dating of north-trending diabase from South Carolina yielded an age of $\sim 200 \mathrm{Ma}$ (Hames et al., 2000), similar to other CAMP volcanic rocks (e.g., 200 Ma ages reported from volcanic rocks of the Fundy Basin in southeastern Canada; Hodych and Dunning, 1992). Age of volcanism in the SGR may be slightly younger than CAMP magmatism to the north, as rifting is thought to have progressively developed southward (Withjack et al., 1998). In addition, volcanic deposits penetrated in southern Georgia interpreted as rift-related include granites, volcanic tuffs, and rhyolites, with granites U-Pb zircon dated at $159 \mathrm{Ma}$ (Heatherington et al., 1999). The volcanic rocks are capped by white and red coarse grained siltstone interpreted as post-rift deposits (Chowns and Williams, 1983). These deposits are labeled as the Cretaceous Cotton Valley Formation, which is also present in the EGOM

\section{Previous Work}

Detrital zircon U-Pb geochronology from previous work by Lovell (2010), Lisi (2013) and Jessee and Weislogel (2014) identified provenance for eolian facies of the Jurassic Norphlet Formation in the EGOM. Dipmeter interpretation by Hunt (2012) determined a net eolian transport direction of south to southeast towards Florida within the Norphlet dune fields in Alabama, which substantially reworked sediments entering the EGOM sub-basins. Mixing between sub-basins was relatively minor. Synthesis of these results reveal that the Norphlet Formation in the southern Alabama onshore is sourced primarily from Laurentian sources, while Gondwanan input increases for the Norphlet Formation toward the southeast in the Pensacola 
Arch, Apalachicola Embayment, and offshore Destin Dome lease block (Fig. 9; Lovell and Weislogel, 2010; Weislogel et al., 2015). Furthermore, results from this study will be used to constrain maximum depositional age (e.g., Dickinson and Gehrels, 2009) of basal units in the EGOM. Basal clastic units in the SFB have been poorly studied, aside from their initial description by Applin and Applin (1965) and Applegate et al. (1981). The age of the Wood River Formation is based on age constraints from whole rock Rb-Sr age of 189 Ma for lower rhyolites, and $138 \mathrm{Ma}$, based on biostratigraphy of overlying carbonate units. The age of Newark Supergroup strata in the SGR is also poorly constrained, and Chowns and Williams (1983) speculated an Upper Jurassic age based on the overlying Upper Jurassic Cotton Valley Formation, the presence of Proterozoic/Paleozoic rock fragments, and basalt intrusions similar to other eastern Laurentian rift-related intrusions. Results from this study will further constrain depositional age based on the maximum depositionl age determined from detrital zircons in SFB and SGR syn-rift deposits. 


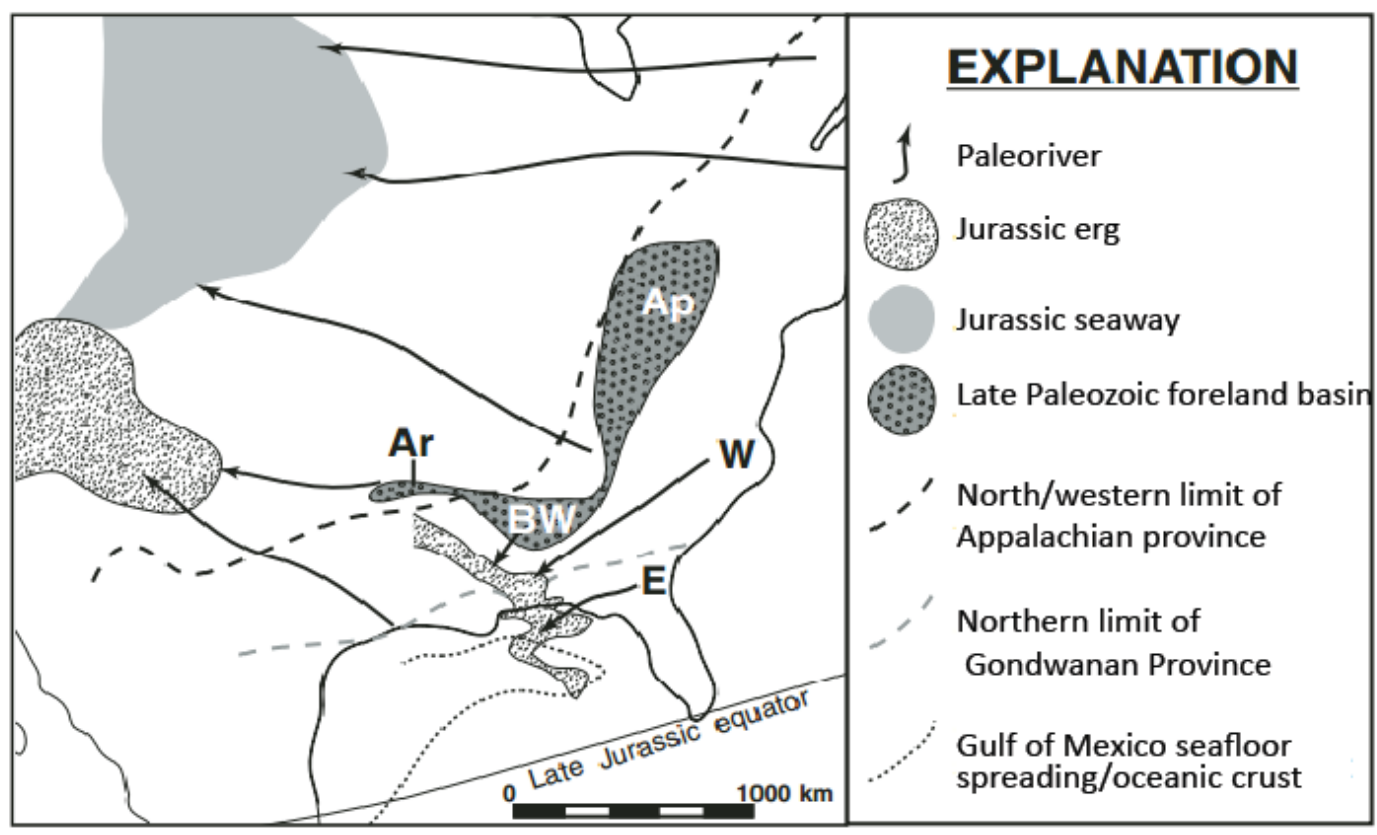

Figure 9. Potential provenance model for the Norphlet Formation in the EGOM. Arrows indicate sediment transport directions for the Norphlet in the EGOM. Two dominant source systems were identified for the Norphlet, a W-western system and the Eeastern system. In addition, sediment may also have been derived from the Ar-Arkoma Basin, BW-Black Warriror Basin, and ApAppalachian Basin. From Weislogel et al. (2015). 


\section{Chapter 3: Methods}

\section{Sample Collection}

Provenance analysis was conducted on a total of 17 samples collected from cuttings and cores. Nine samples were collected from the Florida Geological Survey in March 2015 (Table 1). Sampling targeted the basal sandstone and shale of the Wood River Formation and the basal sandstones found along the southern portion of the Peninsular arch. One additional sample was also collected from syn-rift deposits of the Tallahassee Graben in northern Florida. Due to sampling restrictions, cuttings samples were collected in 3-6 meters (10-20 foot) intervals, with total ranges between 30-120 meters (100-400 feet) thick. Sample size from each 3 meter (10 foot) interval was limited to at most 3.00 grams; sample size ranged from 20-120 grams for each well. Zircon recovery for samples 1411,1655 , and 966 was poor and samples were not dated. Detrital zircon samples were collected from a total of five wells, four which penetrated the basal clastic Wood River $(15095,10566,778,3578)$, and one which likely penetrated the LowerUpper Cretaceous transgressive sandstones/or Paleozoic clastics (2012) (Figure 1).

Three additional samples from offshore southern Florida were acquired from the International Ocean Discovery Program (IODP) Gulf Coast Repository (GCR), at Texas A\&M University in College Station, Texas (Table 2). The samples were collected during Ocean Drilling Project Leg 77, from holes 537 and 538, which penetrated a succession of sandstone and conglomerate, overlying Paleozoic basement. Basement samples collected for this study consist mainly of metamorphosed phylite and igneous intrusions. Sample sizes were less than 100 grams, and of the three samples, only the lower Paleozoic metamorphic basement sample from hole 537 at $200 \mathrm{~m}$ depth yielded zircon grains; this is labeled sample ODP in this study. Samples 
that did not contain zircon were collected from the Creatceous sandy carbonate from hole 537 ( 180 m depth; Unit IV), and the diabase intrusion from hole 538 ( 270 $\mathrm{m} \mathrm{depth})$.

Samples of Triassic Newark Supergroup deposits were collected from cuttings from 5 wells in the SGR from the Georgia Environmental Protection Division core and cutting repository in Rutledge, Georgia in July of 2015 during a visit (Table 3). Samples were manually picked, with approximately 15-20 grams was typically collected every 3 to 6 meters (10-20 foot intervals) until 300-400 grams was collected. Samples of Newark Formation were collected from wells 108, 619, 3137, 3113, and 442 in the SGR. Thin-sections were prepared from cuttings/rock chips collected from wells 442, 108, 619, and 3137. Diabase intrusions are present in several of the wells $(3113,3137,442)$, but previous cutting logs from Chowns and Williams, (1983) were used to avoid the sections.

\section{Detrital Zircon Geochronology}

Cuttings were prepared for zircon analysis following the methods proposed by Gehrels et al. (2014). Separation involved crushing using a disk mill, followed by hydrodynamic separation using a Gemini table if significant sample was present (> 300 grams). Hydraulic separation was performed only on samples of cuttings from Georgia; Florida and IODP samples were too small for hydraulic separation on the Gemini table. After crushing, magnetic separation was performed using a Frantz magnetic separator, followed by liquid separation using lithium polytungstate (LPT) and methlylene iodide (MEI). Samples were soaked in nitric acid to dissolve any pyrite that was present. After separation, the final sample consisted mainly of zircon and barite. Samples were mounted on tape, and non-zircon was manually removed. The zircon was mounted 
using epoxy on circular mounts, polished, and imaged using reflected and transmitted light for zircon dating.

$\mathrm{U}-\mathrm{Pb}$ analysis was completed at the University of South Carolina Center for Elemental Mass Spectrometry in September and December of 2015. Analysis involved laser ablation with a PhotonMachine Analyte G2 1943 nm (deep ultraviolet) ArF exciplex laser to determine ${ }^{206} \mathrm{~Pb} /{ }^{238} \mathrm{U},{ }^{207} \mathrm{~Pb} /{ }^{235} \mathrm{U}$, and ${ }^{235} \mathrm{U}$ to ${ }^{207} \mathrm{~Pb}$ ratios. Spots on sample grains were analyzed using a Photon Machine Analysis using a Thermo Element II ICP-MS. Sample grains were randomly selected from the mount in groups of 5, followed by measurements from each standard. Two standards spots were analyzed, including one from a primary standard grain SL2 Shri Lanka zircon (563.2 \pm 4.8 Ma; Gehrels et al., 2008), and one from a secondary standard 91500 (1065 \pm 0.3 Ma; Wiedenbeck et al., 1995). Analysis standards are used to correct for instrumental offset, and down-hole fractionation. Analysis included an initial $10 \mathrm{~s}$ delay to record background noise, including ${ }^{202} \mathrm{Hg},{ }^{206} \mathrm{~Pb},{ }^{207} \mathrm{~Pb},{ }^{208} \mathrm{~Pb},{ }^{232} \mathrm{Th}$, and ${ }^{238} \mathrm{U}$. Unknown and standard zircon grains were ablated by a $10-\mathrm{Hz}$ laser for 28 seconds, followed by $15 \mathrm{~s}$ of chamber washout. Before running a spot on an unknown sample, three spot analyses were taken on both zircon standards to calibrate the Element II. Both standards were analyzed once between every five unknowns. Between 46125 spots were recorded from single grains for each sample if enough zircons were present.

Data was reduced by Dave Barbeau at the University of South Carolina using the applet $\mathrm{U}-\mathrm{Pb}$ Geochronology 3 data reduction scheme (DRS) of the Iolite software package within IgorPro (Paton et al., 2010). Initial data reduction removes anomalous data points, which included non-zircon/zoned zircons. Ratios of ${ }^{238} \mathrm{U} /{ }^{206} \mathrm{~Pb},{ }^{235} \mathrm{U} /{ }^{207} \mathrm{~Pb},{ }^{232} \mathrm{Th} /{ }^{208} \mathrm{~Pb}$, and ${ }^{207} \mathrm{~Pb} /{ }^{206} \mathrm{~Pb}$ were calculated using the measured counts. Following processing, all grains with 2 standard deviations or greater were removed from the dataset. A concordance filter was applied to all 
grains older than $500 \mathrm{Ma}$ to correct for led loss/gain. Analyses with $<70 \%$ concordance, or $>105 \%$ concordance were removed. Unknowns analyzed with SL2 were selected over the 915 standard due to greater reproducability.

Probability Density Plots (PDP) and Kernal Density Estimates (KDE) were constructed using DensityPlotter (Vermeesch, 2012). A PDP uses the ages and associated error estimates to generate a probability density of the age distribution. In contrast, the KDE assumes Gaussian distribution of ages and associated uncertainty for age sources and creates a smoothed distribution representing the probability of contribution of the age from a source (Vermeesch, 2012). Age distributions were compared with ages known from surrounding tectonic terranes to test for potential sediment sources. U-Th plots were also constructed to identify any metamorphic populations that may have been present to further constrain sediment source. Any zircons with a U/Th ratio over 5 were identified as metamorphic zircons (e.g, Hoskin and Schaltegger, 2003).

Cumulative Density Estimates Plots (CDP) and the K-S test were used to determine if multiple samples may have shared similar sources. The CDP plots age versus the relative probability of an age. It displays the same data as a Probability Density Plot, but individual sample distributions can be plotted together in order to identify changes in probably between age populations within samples.

The K-S is a hypothesis test, which can be used to determine if two samples were derived from the same parent population. The test produces a probability value $(\mathrm{P})$, and $\alpha$ value (level of significance). A Confidence Interval of $95 \%$ and an $\alpha$ value of $5 \%$ were used for this study. $\alpha$ corresponds to 5\% uncertainity in the confidence of the hypothesis test. If the P-value exceeds 
0.05 , then there is $95 \%$ confidence that the two samples are not statistically different and may be derived from the same source. If the P-value is less than 0.05 , then the samples are statistically different, and are likely derived from different sources. The K-S test can be highly biased by degree to which the sample distribution is characterized, particularly samples with diverse zircon ages. The K-S test can also be biased by differences in sample size. Although the majority of the samples from this study had relatively similar sample sizes, some sample sizes were very small, such as sample 10566 which only recovered around 46 zircons. The CDP and K-S test were also used to compare samples between the SFB and the SGR with Norphlet samples from Lisi (2013) and Lovell (2010) to determine if the Norphlet may have shared a similar source with either basin, and if either basin could be a potential sediment pathway into the EGOM.

\section{Thin-Section Petrography}

Thin-Section Petrography was completed to understand variations in lithology and source for the syn-rift clastics. Standard size thin sections $(27 \times 46 \mathrm{~mm})$ were ordered from Spetrum Petrographics Inc and Texas Petrographic Services Inc. Thin sections were impegregnated with a blue epoxy, and stained with Barium Chloride and Rhodizonate for Feldspar. Observations were made using a Nikon Eclipse LV100 microscope using transmitted and reflected light, as well as polarized light. Objectives used include 2X, 4X, 10X, and 20X. A Nikon camera was used for photomicrographs. NIS-Elements software was used to take photos and create figures.

Thin-section point-counting was completed on 12 samples from the SFB to determine tectonic setting (Table 4) using the digitial SteppingState with PetrogLite software. Thin-sections were point-counted for detrital grain composition using the Gazzi-Dickinson method (Dickinson and Suczek, 1979), which identifies and classifies sandstone composition by tectonic source 
based on the abundance of detrital framework grains, such as quartz (monocrystalline or polycrystalline), feldspars (orthoclase and plagioclase), and lithics (metamorphic, igneous, or sedimentary). Identification is based on the composition of individual grains, including quartz, lithics, and feldspar. Point-counting included at least 400 points per sample to limit error of 5\% at 2 standard deviations (Van Der Plas and Tobi, 1965). Compositions were then plotted on a ternary diagram to determine tectonic setting. Thin-sections from the SGR and select SFB samples were too fine-grained for point-counting, but could be used for petrographical observations. In addition to point counting, observations were also recorded on porosity, grain size, sorting, rounding, and other notable features. Overall, 22 thin-sections from the SFB and SGR were examined for this study. Point Counting was used to supplement U-Pb dating to determine provenance. 


\begin{tabular}{|c|c|c|c|c|c|c|c|c|c|c|c|}
\hline $\begin{array}{l}\text { FGS Samples: } \\
\text { Sample name }\end{array}$ & Permit \# & County/State & Well Name & Lat/Long & Sample Type & Lithology & Formation & Depth (ft) & $\begin{array}{l}\text { Operator of } \\
\text { Well }\end{array}$ & Basement & $\begin{array}{l}\text { Zircons } \\
\text { recovered? }\end{array}$ \\
\hline 2012 & 105 & Levy, FL & $\begin{array}{l}\text { Humble Oil and } \\
\text { Refining Co } 1\end{array}$ & $\begin{array}{l}29^{\circ} 4^{\prime} \\
47.99^{\prime \prime} \mathrm{N} / \\
82^{\circ} 37^{\prime} 14^{\prime \prime} \\
\mathrm{W}\end{array}$ & Cuttings & $\begin{array}{l}\text { red } \\
\text { sandstone/shale }\end{array}$ & $\begin{array}{l}\text { Paleozoic/Lower } \\
\text { Mesozoic clastics }\end{array}$ & $4000-4370$ & Humble Oil & Serpentinite & Yes \\
\hline 3578 & 225 & Highlands, FL & $\begin{array}{l}\text { Continental } \\
\text { Carleton }\end{array}$ & $\begin{array}{l}27^{\circ} 9^{\prime} 34^{\prime \prime} \\
\mathrm{N} / 81^{\circ} 32^{\prime} \\
9^{\prime \prime} \mathrm{W}\end{array}$ & Cuttings & $\begin{array}{l}\text { dolomite, shale, } \\
\text { uncommon } \\
\text { sandstone }\end{array}$ & $\begin{array}{l}\text { Wood River/lower } \\
\text { Cretaceous }\end{array}$ & $\begin{array}{l}12130- \\
12550\end{array}$ & Continental Oil & $\begin{array}{l}\text { Metamorphic } \\
\text { shale/Basalt }\end{array}$ & Yes \\
\hline 10566 & 407 & Lee, FL & $\begin{array}{l}\text { Humble oil Lehigh } \\
\text { Acres }\end{array}$ & $\begin{array}{l}26^{\circ} 33^{\prime} 38^{\prime \prime} \\
\mathrm{N} / 81^{\circ} 35^{\prime} \\
5^{\prime \prime} \mathrm{W}\end{array}$ & Cuttings & $\begin{array}{l}\text { red } \\
\text { sandstone/shale/ } \\
\text { dolomite }\end{array}$ & Wood River & $1550-15660$ & Penrod Drilling & Quartz diabase & Yes \\
\hline 15095 & unknown & Collier, FL & $\begin{array}{l}\text { Exxon Corp } \\
\text { Sunniland Field }\end{array}$ & $\begin{array}{l}26^{\circ} 17^{\prime} 29^{\prime \prime} \\
\mathrm{N} / 81^{\circ} 20^{\prime} \\
51^{\prime \prime} \mathrm{W}\end{array}$ & $\begin{array}{l}\text { Cuttings/core } \\
\text { chips }\end{array}$ & red sandstone & Wood River & $\begin{array}{l}16802- \\
16949\end{array}$ & Exxon & $\begin{array}{l}\text { Rhyolite } \\
\text { Porphyry }\end{array}$ & Yes \\
\hline 1854 & 95 & Jefferson, FL & E.P Larsh \#1 & $\begin{array}{l}30^{\circ} 20^{\prime} \\
15.648^{\prime \prime} \mathrm{N} / \\
81^{\circ} 58^{\prime} 296^{\prime \prime} \\
\mathrm{W}\end{array}$ & Cuttings & sandstone & Basal sandstone & $7280-7610$ & $\begin{array}{l}\text { Coastal } \\
\text { Petroleum } \\
\text { Company }\end{array}$ & Did not penetrate & Yes \\
\hline 778 & 778 & Collier, FL & Collier Co. \#12-2 & $\begin{array}{l}25^{\circ} 57^{\prime} 31^{\prime \prime} \\
\mathrm{N} / 81^{\circ} 33^{\prime} \\
49^{\prime \prime} \mathrm{W}\end{array}$ & Cuttings & sandstone & Wood River & $\begin{array}{l}18300- \\
18640\end{array}$ & $\begin{array}{l}\text { Hodges Well } \\
\text { Drilling }\end{array}$ & Did not penetrate & Yes \\
\hline 1411 & 31 & Osceola, FL & $\begin{array}{l}\text { W.P Hayman- } \\
\text { Apoxsee }\end{array}$ & $\begin{array}{l}27^{\circ} 47^{\prime} \\
25.74^{\prime \prime} / 80^{\circ} \\
58^{\prime} 43^{\prime \prime}\end{array}$ & Cuttings & $\begin{array}{l}\text { sandy dolomite, } \\
\text { red } \\
\text { sandstone/shale }\end{array}$ & $\begin{array}{l}\text { Wood River/lower } \\
\text { Cretaceous section }\end{array}$ & $8540-8775$ & $\begin{array}{l}\text { Loffland } \\
\text { Brothers Co }\end{array}$ & Rhyolite & $\begin{array}{l}\text { Insufficient } \\
\text { for Dating }\end{array}$ \\
\hline 1655 & 62 & Hardee, FL & B.T. Keen No. 1 & $\begin{array}{l}27^{\circ} 24^{\prime} \\
47.03^{\prime \prime} / 81^{\circ} \\
59^{\prime} 3.5^{\prime \prime}\end{array}$ & Cuttings & $\begin{array}{l}\text { dolomite, shale, } \\
\text { and sandstone }\end{array}$ & Wood River & $\begin{array}{l}11450- \\
11800\end{array}$ & Lottland & Basalt & $\begin{array}{l}\text { Insufficient } \\
\text { for Dating }\end{array}$ \\
\hline 966 & 225 & Highlands, FL & C.C. Carlton Est. 1 & $\begin{array}{l}27^{\circ} 7^{\prime} 46^{\prime \prime} / \\
81^{\circ} 24^{\prime} 16^{\prime \prime}\end{array}$ & Cuttings & $\begin{array}{l}\text { dolomite, some } \\
\text { shale/sandstone }\end{array}$ & $\begin{array}{l}\text { Wood River/Creataceous } \\
\text { clastics }\end{array}$ & $\begin{array}{l}12040- \\
12250\end{array}$ & Humble Oil & Quartzite & No \\
\hline
\end{tabular}




\begin{tabular}{|c|c|c|c|c|c|c|c|c|c|}
\hline $\begin{array}{l}\text { EPD Samples: Sample } \\
\text { Name/ GGS Number }\end{array}$ & Well Name & $\begin{array}{l}\text { County } \\
\text { /State }\end{array}$ & & $\begin{array}{l}\text { Sample } \\
\text { type }\end{array}$ & Lithology & Formation & Depth (ft) & $\begin{array}{l}\text { Operat of } \\
\text { Well }\end{array}$ & $\begin{array}{l}\text { Zircon } \\
\text { Recovered? }\end{array}$ \\
\hline 52 & $\begin{array}{l}\text { Brunswick } \\
\text { Peninsular } \\
\text { Corp \#1 }\end{array}$ & $\begin{array}{l}\text { Wayne, } \\
\text { GA }\end{array}$ & $\begin{array}{l}3123^{\prime} 28^{\prime \prime} / \\
-8148^{\prime} 22^{\prime \prime}\end{array}$ & Cuttings & $\begin{array}{l}\text { Arkosic } \\
\text { sandstone }\end{array}$ & Newark Group & $4560-4630$ & $\begin{array}{l}\text { California Oil } \\
\text { Co }\end{array}$ & Yes, but not dated \\
\hline 108 & Cecil Pate \#1 & $\begin{array}{l}\text { Crisp, } \\
\text { GA }\end{array}$ & $\begin{array}{l}3149^{\prime} 35^{\prime \prime /} \\
-8346^{\prime} 10^{\prime \prime}\end{array}$ & Cuttings & Sandstone/shale & Newark Group & $4820-5010$ & Kerr McGee Oil & Yes \\
\hline 442 & $\begin{array}{l}\text { Walter F } \\
\text { Stevens \#1 }\end{array}$ & $\begin{array}{l}\text { Sumter, } \\
\text { GA }\end{array}$ & $\begin{array}{l}3201^{\prime} 05^{\prime \prime /} \\
-8418^{\prime} 30^{\prime \prime}\end{array}$ & Cuttings & $\begin{array}{l}\text { Arkosic } \\
\text { sandstone and } \\
\text { red shale }\end{array}$ & Newark Group & $3980-4520$ & Flinn Austin Co & Yes \\
\hline 619 & $\begin{array}{l}\text { H E Walton } \\
\# 1\end{array}$ & $\begin{array}{l}\text { Dooly, } \\
\text { GA }\end{array}$ & $\begin{array}{l}3202^{\prime} 29^{\prime \prime} / \\
-8339^{\prime} 00^{\prime \prime}\end{array}$ & Cuttings & $\begin{array}{l}\text { Arkosic } \\
\text { sandstone }\end{array}$ & Newark Group & $3512-3750$ & $\begin{array}{l}\text { Georgia Florida } \\
\text { Drilling Co }\end{array}$ & Yes \\
\hline 3113 & $\begin{array}{l}\text { J. T. Stalvey } \\
\# 1\end{array}$ & $\begin{array}{l}\text { Lownde, } \\
\text { GA }\end{array}$ & $\begin{array}{l}3259^{\prime} 259^{\prime \prime} / \\
-8315^{\prime} 08^{\prime \prime}\end{array}$ & Cuttings & $\begin{array}{l}\text { Arkosic } \\
\text { sandstone, red } \\
\text { and black shale, } \\
\text { and diabase }\end{array}$ & Newark Group & $8130-8510$ & Hunt Oil Co. & Yes \\
\hline 3137 & Griffiths \#1 & $\begin{array}{l}\text { Pulaski, } \\
\text { GA }\end{array}$ & $\begin{array}{l}3219^{\prime} 32 " / \\
-8315^{\prime} 08^{\prime \prime}\end{array}$ & Cuttings & $\begin{array}{l}\text { Red sand and } \\
\text { shale }\end{array}$ & Newark Group & $5320-5827$ & $\begin{array}{l}\text { Atlanta Gas Co, } \\
\text { S }\end{array}$ & Yes \\
\hline 3154 & Key\#1 & $\begin{array}{l}\text { Worth, } \\
\text { GA }\end{array}$ & unknown & Cuttings & Red sandy shale & Newark Group & $5465-5675$ & So. Inv. & Yes, but not dated \\
\hline
\end{tabular}

Table 2. Samples collected from the Georgia Geological Survey.

\begin{tabular}{|l|l|l|l|l|l|l|}
\hline $\begin{array}{l}\text { ODP Sample: Sample } \\
\text { Name }\end{array}$ & Expedition & Site & Lat/Long & Depth interval & $\begin{array}{l}\text { Lithology } \\
\text { Recovered } \\
\text { ? }\end{array}$ \\
\hline ODP & 77 & 0537 & $\begin{array}{l}23^{\circ} 56.010 \mathrm{~N} / \\
85^{\circ} 27.618 \mathrm{~W}\end{array}$ & $22-24.5$ & Phylite & Yes \\
\hline ODP-2 & 77 & 0537 & $\begin{array}{l}23^{\circ} 56.010 \mathrm{~N} / \\
85^{\circ} 27.618 \mathrm{~W}\end{array}$ & $95-110$ & $\begin{array}{l}\text { Carbonate } \\
\text { cement with } \\
\text { quartz and } \\
\text { perthite grains }\end{array}$ & No \\
\hline ODP-3 & 77 & 0538 & $\begin{array}{l}23^{\circ} 50.98^{\prime} \mathrm{N} ; \\
85^{\circ} 10.26^{\prime} \mathrm{W}\end{array}$ & $54.57-57$ & diabase & No \\
\hline
\end{tabular}

Table 3. ODP samples collected for this study. 


\section{Logs}

Wireline well logs for 15 wells provided by the Florida Geological Survey were evaluated to identify and map the extent of the Wood River Formation/Peninsular arch basal sands in the subsurface. Paper logs were uploaded, georeferenced, and digitized in Petra. Logs included gamma ray, caliper, spontaneous potential, resistivity, neutron and density porosity. Logs were digitized in Petra, and tops were created based on driller's logs and electronic log curves. A map of the basal clastic tops and basement was created to identify the general orientation in the South Florida Basin. An isochore map was created to determine thickness variations. V-shale was calculated from gamma ray to identify shale content, and lateral variations in lithology. Regions of low v-shale may correlate to more proximal parts of the basin, while higher v-shale may correlate to more distal. 


\begin{tabular}{|c|c|c|c|c|c|c|c|c|}
\hline Sample \# & Well Name & $\begin{array}{l}\text { County/ } \\
\text { State }\end{array}$ & Lat/Long & Sample Type & Lithology & Formation & Depth (ft) & $\begin{array}{l}\text { Point } \\
\text { Count? }\end{array}$ \\
\hline \multicolumn{9}{|l|}{$\underline{\underline{S F B}}$} \\
\hline $778-1$ & $\begin{array}{l}\text { Collier Co } \\
\# 12-2\end{array}$ & Collier, FL & $\begin{array}{l}25^{\circ} 57^{\prime} 31^{\prime \prime} \\
\mathrm{N} / 81^{\circ} 33^{\prime} \\
49^{\prime \prime} \mathrm{W}\end{array}$ & $\begin{array}{l}\text { cuttings/core } \\
\text { chips }\end{array}$ & Sandstone/shale/limestone & Wood River & $\begin{array}{l}17800- \\
17900\end{array}$ & No \\
\hline $778-2$ & $\begin{array}{l}\text { Collier Co } \\
\# 12-2\end{array}$ & Collier, FL & $\begin{array}{l}25^{\circ} 57^{\prime} 31^{\prime \prime} \\
\mathrm{N} / 81^{\circ} 33^{\prime} \\
49^{\prime \prime} \mathrm{W}\end{array}$ & $\begin{array}{l}\text { Cuttings/core } \\
\text { chips }\end{array}$ & sandstone & Wood River & $\begin{array}{l}18400- \\
18410\end{array}$ & Yes \\
\hline ODP-1 & Site 0537 & $\begin{array}{l}\text { Offshore } \\
\text { EGOM }\end{array}$ & $\begin{array}{l}23^{\circ} 56.01^{\prime} \mathrm{N} / \\
85^{\circ} 27.62^{\prime} \mathrm{W}\end{array}$ & core & Sandstone/carbonate & unnamed & $\begin{array}{l}\text { Interval } \\
95-110\end{array}$ & No \\
\hline ODP-2 & Site 0538. & $\begin{array}{l}\text { Offshore } \\
\text { EGOM }\end{array}$ & $\begin{array}{l}23^{\circ} 50.98^{\prime} \mathrm{N} ; \\
85^{\circ} 10.26^{\prime} \mathrm{W}\end{array}$ & core & diabase & unnamed & $\begin{array}{l}\text { Interval } \\
54.57-57\end{array}$ & No \\
\hline 10566 & $\begin{array}{l}\text { Humble } \\
\text { Oil Lehigh } \\
\text { Acres }\end{array}$ & Lee, FL & $\begin{array}{l}27^{\circ} 9^{\prime} 34^{\prime \prime} \mathrm{N} / \\
81^{\circ} 32^{\prime} 9^{\prime \prime} \mathrm{W}\end{array}$ & cuttings & sandstone & Wood River & 15708 & Yes \\
\hline 1411 & $\begin{array}{l}\text { Jean } \\
\text { McArthur } \\
\text { Davis 9-3 }\end{array}$ & $\begin{array}{l}\text { Okeechobee, } \\
\text { FL }\end{array}$ & $\begin{array}{l}27^{\circ} 26^{\prime} \\
17.063^{\prime \prime} \\
\text { N/ } 80^{\circ} 50^{\prime} \\
17.07^{\prime \prime} \mathrm{W}\end{array}$ & cuttings & sandstone & $\begin{array}{l}\text { Wood River } \\
\text { (?)/proximal } \\
\text { clastics }\end{array}$ & 10560 & Yes \\
\hline 1655 & $\begin{array}{l}\text { B.T Keen } \\
\text { ET UX\#1 }\end{array}$ & Hardee, FL & $\begin{array}{l}27^{\circ} 24^{\prime} \\
47.03^{\prime \prime} \mathrm{N} / 81^{\circ} \\
59^{\prime} 3.5^{\prime \prime}\end{array}$ & cuttings & sandstone & $\begin{array}{l}\text { Wood River } \\
\text { (?)/proximal } \\
\text { clastics }\end{array}$ & 11930 & No \\
\hline $2012-1$ & $\begin{array}{l}\text { Humble } \\
\text { Oil and } \\
\text { Refining } \\
\text { Co \#1 } \\
\end{array}$ & Levy, FL & $\begin{array}{l}29^{\circ} 4^{\prime} \\
48.0684^{\prime \prime} \mathrm{N} / \\
82^{\circ} 37^{\prime} \\
14.1168 \mathrm{~W} \\
\end{array}$ & cuttings & sandstone & $\begin{array}{l}\text { Wood River } \\
(?) / \text { proximal } \\
\text { clastics }\end{array}$ & $\begin{array}{l}4305- \\
4310\end{array}$ & Yes \\
\hline $2012-2$ & $\begin{array}{l}\text { Humble } \\
\text { Oil and } \\
\text { Refining } \\
\text { Co \#1 }\end{array}$ & Levy, FL & $\begin{array}{l}29^{\circ} 4^{\prime} \\
48.0684^{\prime \prime} \mathrm{N} / \\
82^{\circ} 37^{\prime} \\
14.1168 \mathrm{~W}\end{array}$ & cuttings & sandstone & $\begin{array}{l}\text { Wood River } \\
\text { (?)/proximal } \\
\text { clastics }\end{array}$ & $\begin{array}{l}4384- \\
4391\end{array}$ & Yes \\
\hline $3578-1$ & $\begin{array}{l}\text { Continental } \\
\text { Coil } \\
\text { Company } \\
\text { well }\end{array}$ & $\begin{array}{l}\text { Highlands, } \\
\text { FL }\end{array}$ & $\begin{array}{l}27^{\circ} 9^{\prime} 34^{\prime \prime} \mathrm{N} / \\
81^{\circ} 32^{\prime} 9^{\prime \prime} \mathrm{W}\end{array}$ & cuttings & shale & $\begin{array}{l}\text { Wood River } \\
\text { (?)/proximal } \\
\text { clastics }\end{array}$ & $\begin{array}{l}12550- \\
12554\end{array}$ & No \\
\hline $3578-2$ & $\begin{array}{l}\text { Continental } \\
\text { Coil } \\
\text { Company } \\
\text { well } \\
\end{array}$ & $\begin{array}{l}\text { Highlands, } \\
\text { FL }\end{array}$ & $\begin{array}{l}27^{\circ} 9^{\prime} 34^{\prime \prime} \mathrm{N} / \\
81^{\circ} 32^{\prime} 9^{\prime \prime} \mathrm{W}\end{array}$ & cuttings & sandstone & $\begin{array}{l}\text { Wood River } \\
(?) / \text { proximal } \\
\text { clastics }\end{array}$ & $\begin{array}{l}12600- \\
12614\end{array}$ & Yes \\
\hline $3578-3$ & $\begin{array}{l}\text { Continental } \\
\text { Coil } \\
\text { Company } \\
\text { well }\end{array}$ & $\begin{array}{l}\text { Highlands, } \\
\text { FL }\end{array}$ & $\begin{array}{l}27^{\circ} 9^{\prime} 34^{\prime \prime} \mathrm{N} / \\
81^{\circ} 32^{\prime} 9^{\prime \prime} \mathrm{W}\end{array}$ & cuttings & sandstone & $\begin{array}{l}\text { Wood River } \\
\text { (?)/proximal } \\
\text { clastics }\end{array}$ & $\begin{array}{l}12614- \\
12629\end{array}$ & Yes \\
\hline $966-1$ & $\begin{array}{l}\text { Carlton } \\
\text { Est. \#1 }\end{array}$ & $\begin{array}{l}\text { Highlands, } \\
\text { FL }\end{array}$ & $\begin{array}{l}27^{\circ} 9^{\prime} 34^{\prime \prime} \mathrm{N} / \\
81^{\circ} 32^{\prime} 9^{\prime \prime} \mathrm{W}\end{array}$ & cuttings & sandstone & $\begin{array}{l}\text { Wood River } \\
(?) / \text { proximal } \\
\text { clastics } \\
\end{array}$ & 12256 & Yes \\
\hline $966-2$ & $\begin{array}{l}\text { Carlton } \\
\text { Est. \#1 }\end{array}$ & $\begin{array}{l}\text { Highlands, } \\
\text { FL }\end{array}$ & $\begin{array}{l}27^{\circ} 9^{\prime} 34^{\prime \prime} \mathrm{N} / \\
81^{\circ} 32^{\prime} 9^{\prime \prime} \mathrm{W}\end{array}$ & cuttings & sandstone & $\begin{array}{l}\text { Wood River } \\
\text { (?)/proximal } \\
\text { clastics }\end{array}$ & 12288 & Yes \\
\hline $966-3$ & $\begin{array}{l}\text { Carlton } \\
\text { Est. \#1 }\end{array}$ & $\begin{array}{l}\text { Highlands, } \\
\text { FL }\end{array}$ & $\begin{array}{l}27^{\circ} 9^{\prime} 34^{\prime \prime} \mathrm{N} / \\
81^{\circ} 32^{\prime} 9^{\prime \prime} \mathrm{W}\end{array}$ & cuttings & sandstone & $\begin{array}{l}\text { Wood River } \\
\text { (?)/proximal } \\
\text { clastics }\end{array}$ & $\begin{array}{l}12235- \\
12240\end{array}$ & Yes \\
\hline $966-4$ & $\begin{array}{l}\text { Carlton } \\
\text { Est. \#1 }\end{array}$ & $\begin{array}{l}\text { Highlands, } \\
\text { FL }\end{array}$ & $\begin{array}{l}27^{\circ} 9^{\prime} 34^{\prime \prime} \mathrm{N} / \\
81^{\circ} 32^{\prime} 9^{\prime \prime} \mathrm{W}\end{array}$ & cuttings & sandstone & $\begin{array}{l}\text { Wood River } \\
\text { (?)/proximal } \\
\text { clastics }\end{array}$ & $\begin{array}{l}12246- \\
12249\end{array}$ & Yes \\
\hline \multicolumn{9}{|l|}{ SGR } \\
\hline$\overline{442-1}$ & $\begin{array}{l}\text { Walther F } \\
\text { Stevens \#1 }\end{array}$ & Sumter, GA & $\begin{array}{l}32^{\circ} 01^{\prime} 05^{\prime \prime} \\
\mathrm{N} / 84^{\circ} 13^{\prime} \\
30^{\prime \prime} \mathrm{W}\end{array}$ & cuttings & shale & Newark & $\begin{array}{l}4120- \\
4130\end{array}$ & No \\
\hline $442-2$ & $\begin{array}{l}\text { Walther F } \\
\text { Stevens \#1 }\end{array}$ & Sumter, GA & $\begin{array}{l}32^{\circ} 01^{\prime} 05^{\prime \prime} \\
\mathrm{N} / 84^{\circ} 13^{\prime} \\
30^{\prime \prime} \mathrm{W}\end{array}$ & cuttings & shale & Newark & $\begin{array}{l}4440- \\
4450\end{array}$ & No \\
\hline 108 & $\begin{array}{l}\text { Cecil Plate } \\
\# 1\end{array}$ & Grisp, GA & $\begin{array}{l}31^{\circ} 49^{\prime} 35^{\prime \prime} \\
\mathrm{N} / 83^{\circ} 46^{\prime} \\
10^{\prime \prime} \mathrm{W}\end{array}$ & cuttings & sandy shale & Newark & $\begin{array}{l}4860- \\
4870\end{array}$ & No \\
\hline 619 & $\begin{array}{l}\text { H. E } \\
\text { Walton \#1 }\end{array}$ & Dooly, GA & $\begin{array}{l}32^{\circ} 02^{\prime} 30^{\prime \prime} \\
\mathrm{N} / 83^{\circ} 39^{\prime} \\
00^{\prime \prime} \mathrm{W}\end{array}$ & cuttings & sandy shale & Newark & $\begin{array}{l}3770- \\
3780\end{array}$ & No \\
\hline $3137-1$ & Griffiths \#1 & Pulaski, GA & $\begin{array}{l}32^{\circ} 19^{\prime} 32^{\prime \prime} \\
\mathrm{N} / 83^{\circ} 32^{\prime} \\
27^{\prime \prime} \mathrm{W}\end{array}$ & cuttings & shale & Newark & $\begin{array}{l}5630- \\
5640\end{array}$ & No \\
\hline $3137-2$ & Griffiths \#1 & Pulaski, GA & $\begin{array}{l}32^{\circ} 19^{\prime} 32^{\prime \prime} \\
\mathrm{N} / 83^{\circ} 32^{\prime} \\
27^{\prime \prime} \mathrm{W}\end{array}$ & cuttings & Sandy shale & Newark & $\begin{array}{l}5440- \\
5450\end{array}$ & No \\
\hline
\end{tabular}

Table 4. Thin sections collected for this study. 


\section{Chapter 4: RESULTS}

\section{Florida Detrital Zircon Ages}

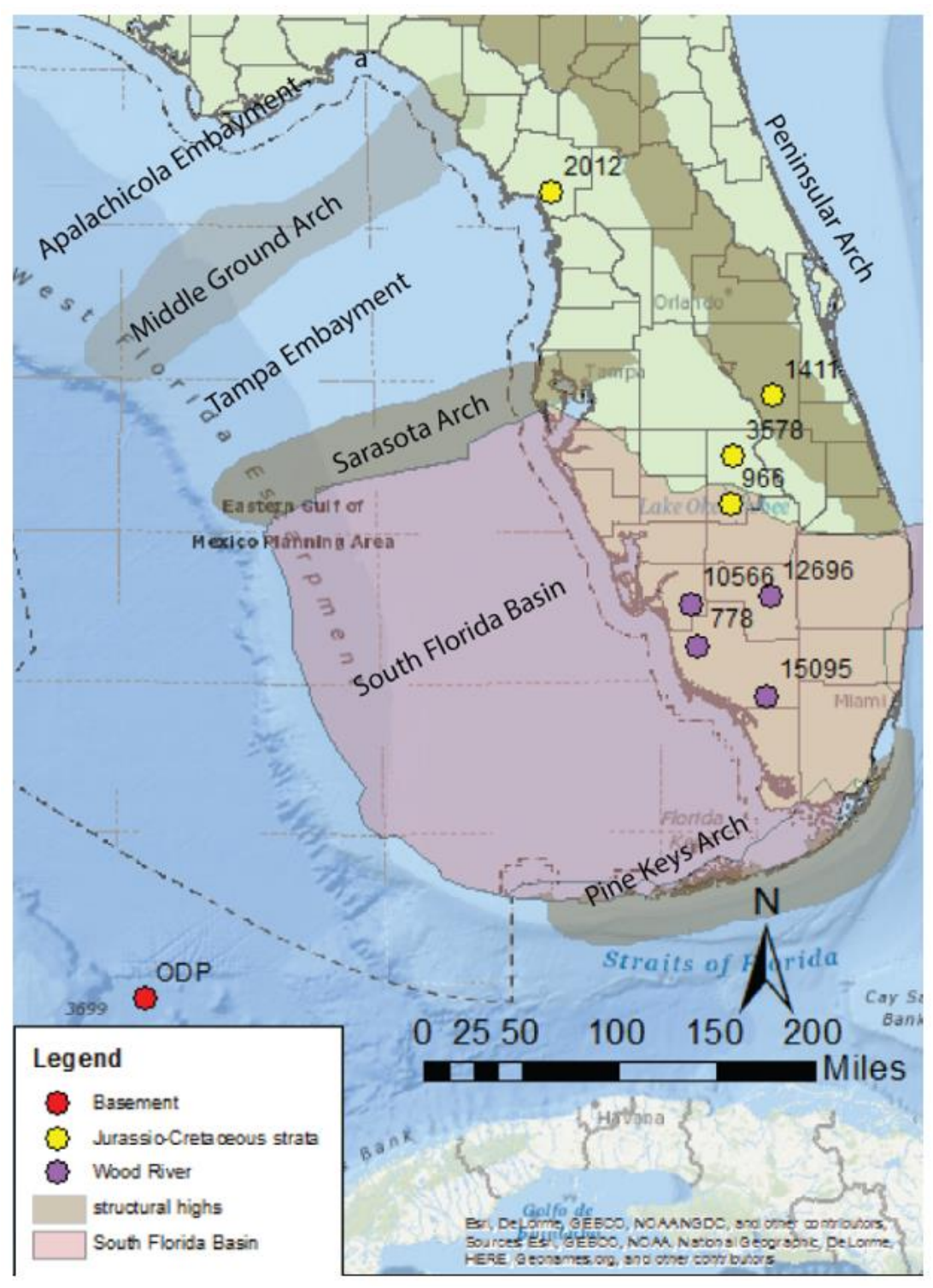

Figure 10. Location of samples collected from Florida. Samples 778, 10566, and 15095 are from the Wood River Formation. Sample 3578 likely represents correlative facies of the Wood River Formation, or Jurassic-Creatceous transgressive strata. Sample 2012 was collected from central Florida strata of Paleozoic or Jurassic/Cretaceous origin. ODP was collected from offshore basement. No zircons were recovered from 12696, 966, or 1411.
A map of samples collected

from South Florida is displayed in

Figure 10. Zircon grains were recovered from samples 15095,778 , 10566, 3578, 2012, and ODP. Samples 15095, 778, and 10566 were collected from Wood River Formation clastic deposits, initially described in Applegate et al. (1981). Lithology of sample 3578 was similar to the Wood River Formation, and is likely a continuation of the Wood River, or Upper Jurassic/Lower Creatceous Transgressive clastic deposits. Sample 2012 is from central Florida Suwannee Basin, and is likely Paleozoic or Triassic/Jurassic in age (Mueller, et al., 1994; Boote and Knapp, 2016). Sample ODP was collected from the

offshore IODP hole 537, and was collected from phylite basement rocks. 


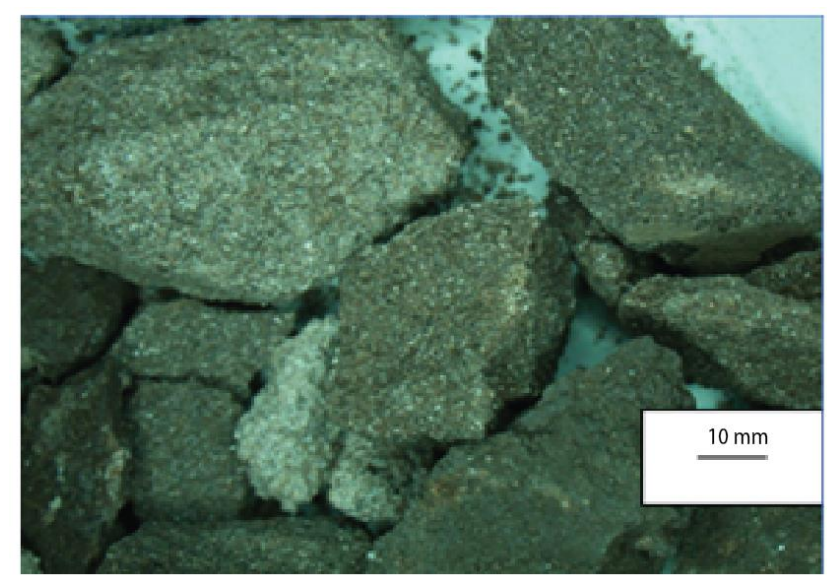

Figure 11. Sample 15095, collected from rock chips, is composed of coarse-grained sandstone and limestone.
Sample 15095 was collected from well 15095 from Collier county Florida from cuttings and core chips $(16,802-16,949$ feet $)$, for a total sample mass of $\sim 56$ grams. The section consists primarily of coarse-grained red sandstone (Fig. 11).

In all, 108 zircons were analyzed; of the 108, 17 analyses were removed due to high uncertainty and/or discordance (Fig. 12). Zircon ages range from $194+/-$ $4 \mathrm{Ma}$ to 2,321 +/- $215 \mathrm{Ma}$. There is one main population of ages between 419 to $694 \mathrm{Ma}(90.5 \%$ of all ages) with a peak at $486 \mathrm{Ma}$.

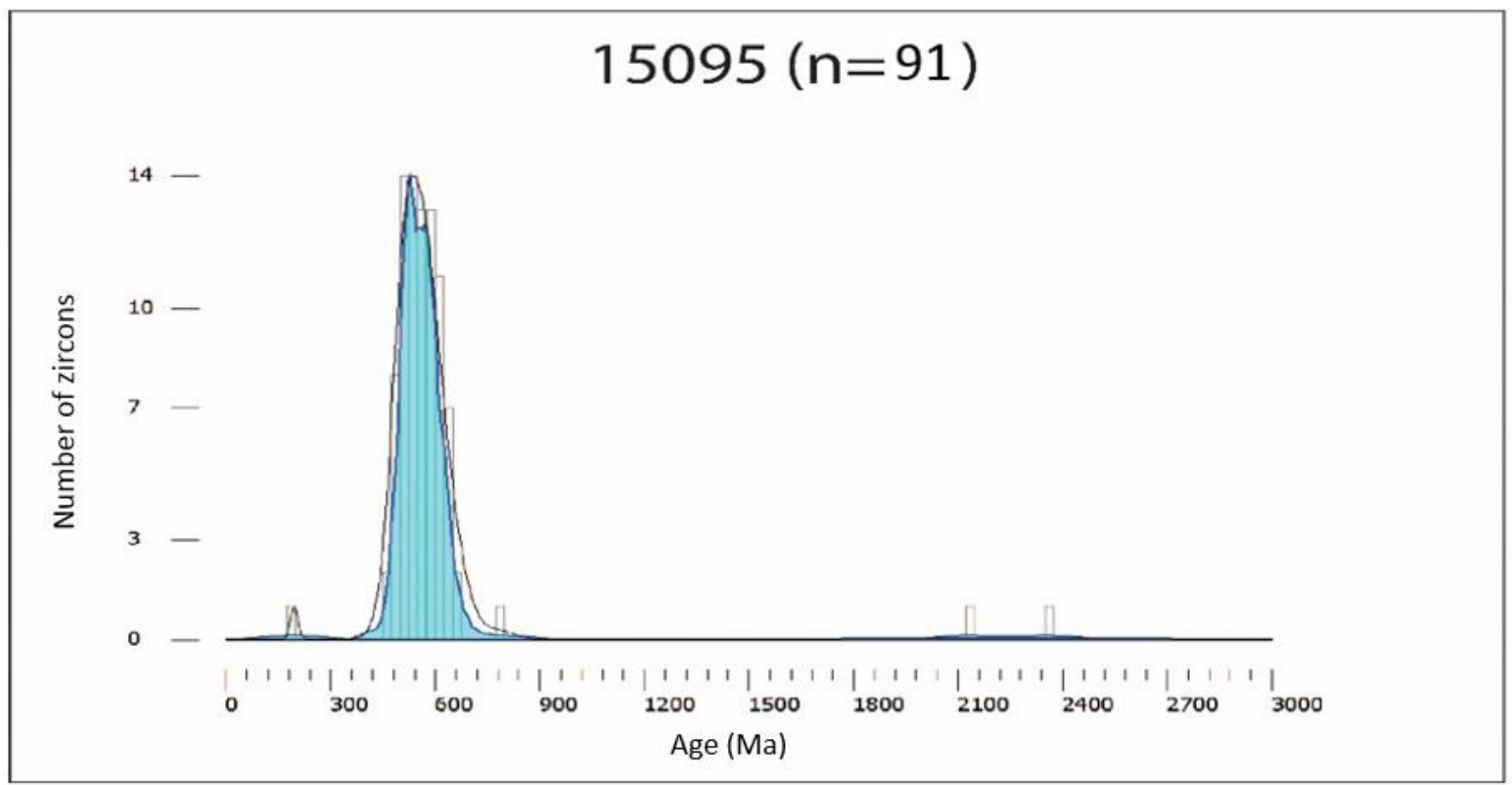

Figure 12. PDP (black line) and KDE (blue) from sample 15095. The $X$ axis is age (Ma), and the $Y$ axis is number of grains. 
Sample 3578 Wood River/ Triassic-mid Cretaceous proximal sandstone?

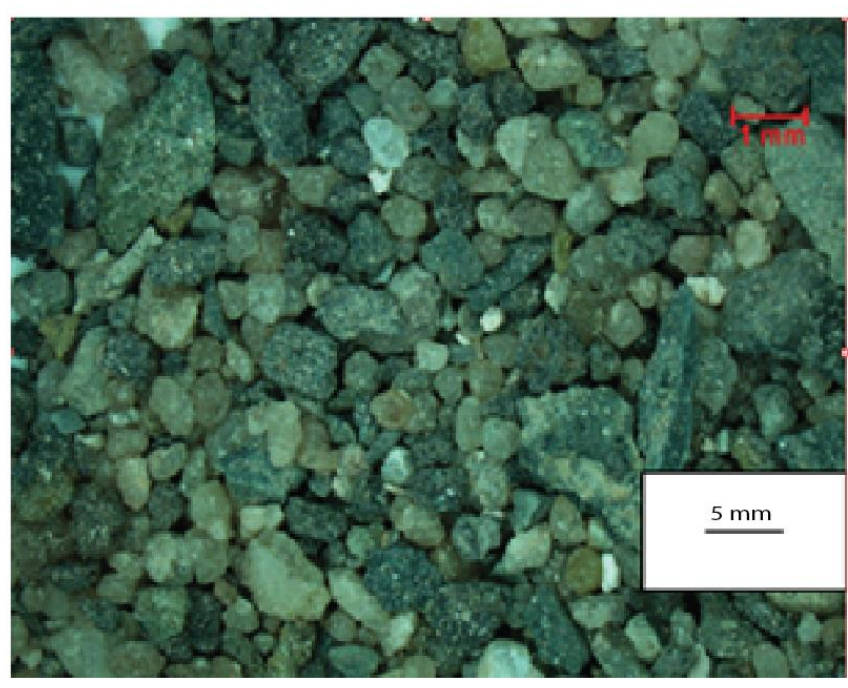

Figure 13. Sample 3578, collected from cuttings, is composed of coarse grained sand, limestone and shale.
Sample 3578 was collected from well 3578 (permit 225) from Highlands county, Florida. The sample was collected from cuttings between 12,130-12,550 feet, yielding $\sim 86$ grams. The base of the section consists primarily of sandstone, which grades vertically into shale, and dolomite/limestone (Fig. 13).

In all, 119 zircons were analyzed.

Eleven were removed due to high uncertainty and/or discordance (Fig. 14). Ages range from $426 \mathrm{Ma}+/-11 \mathrm{Ma}$ to 2,732 +/- 40.5 Ma. Most ages range from 510 to $718 \mathrm{Ma}(92 \%)$. The major peak of this population is $580 \mathrm{Ma}$.

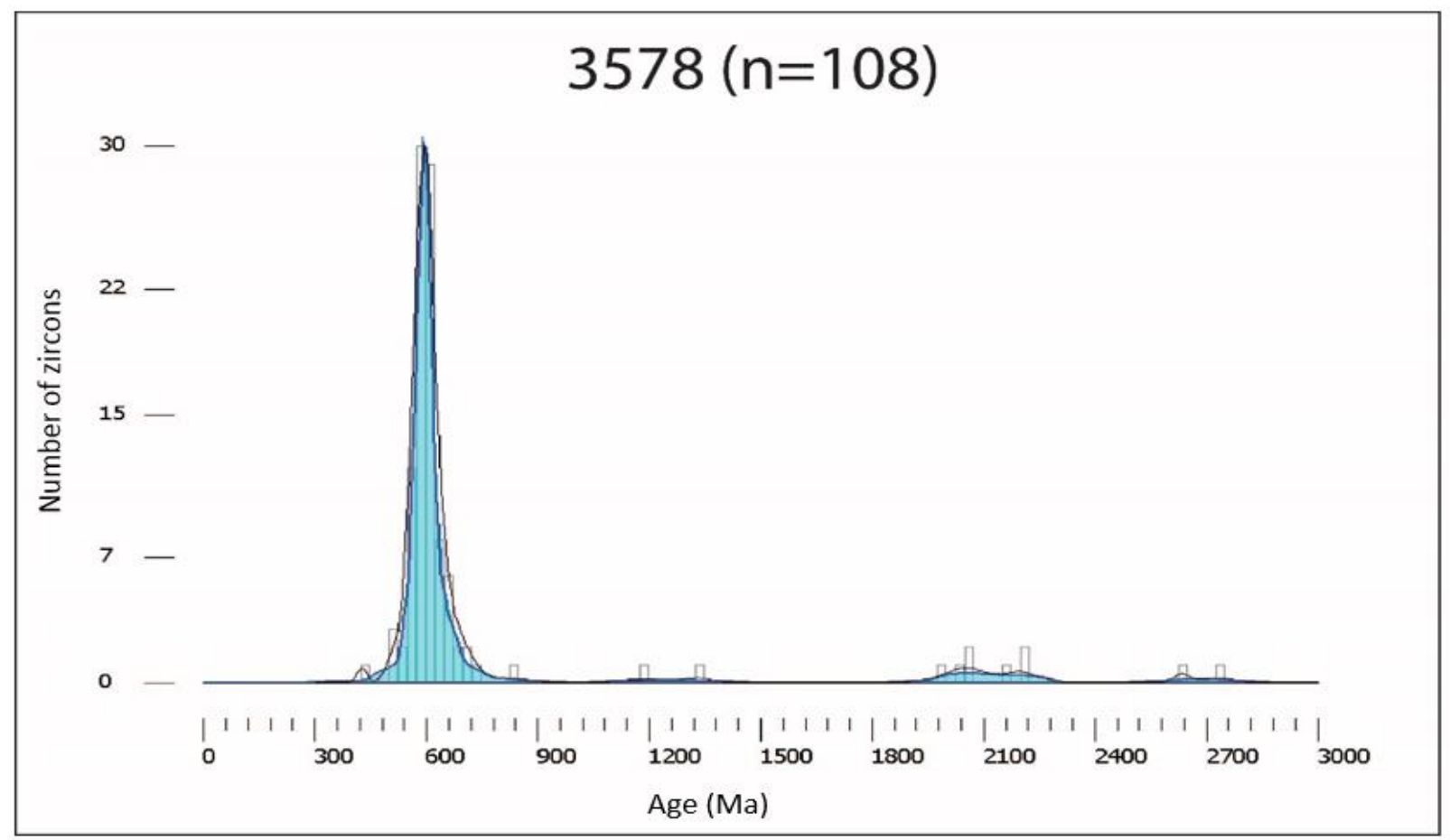

Figure 14. PDP (black line) and KDE (blue) from sample 3578. The $X$ axis is age (Ma), and the $Y$ axis is number of grains. 
Sample 778 Triassic-Jurassic (?) Wood River

Sample 778 was collected from Well 12838 (permit number 778) from Collier county, Florida.

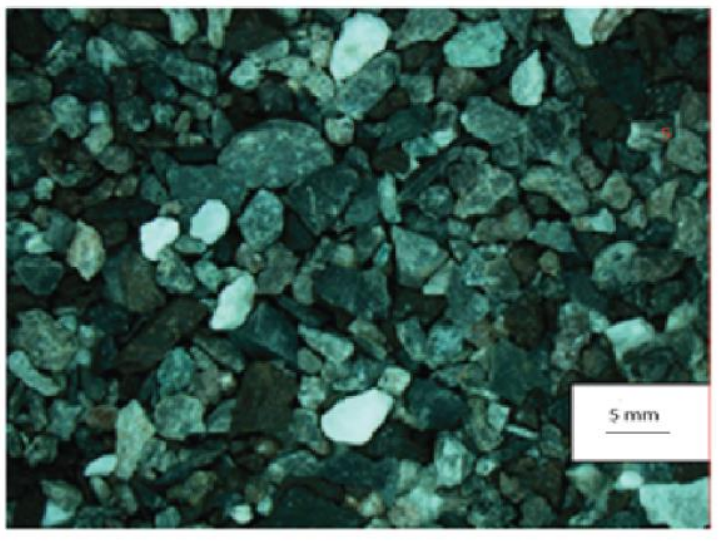

Figure 15. Sample 778, collected from cuttings, is composed of mixed anhydrite, sand, and limestone.
Sample size was $\sim 45$ grams of cuttings collected from 18,300-18,640 feet depth. The sample consists predominantly of red, medum-grained sandstone and coarse siltstone. The upper section grades upward into a limestone/dolomite (Fig. 15).

In all, 112 ages were analyzed. Three ages were removed due to high uncertainty and/or high discordance. Ages range from $201+/-4 \mathrm{Ma}$, to

2,673 +/- $16 \mathrm{Ma}$. Most ages range from 450 to $650 \mathrm{Ma}(79 \%)$ with a major age peak at $550 \mathrm{Ma}$, and a much smaller population ranges from 2,000-2,200 Ma (7.3\%) with a peak at 2,016 Ma (Fig. 16).

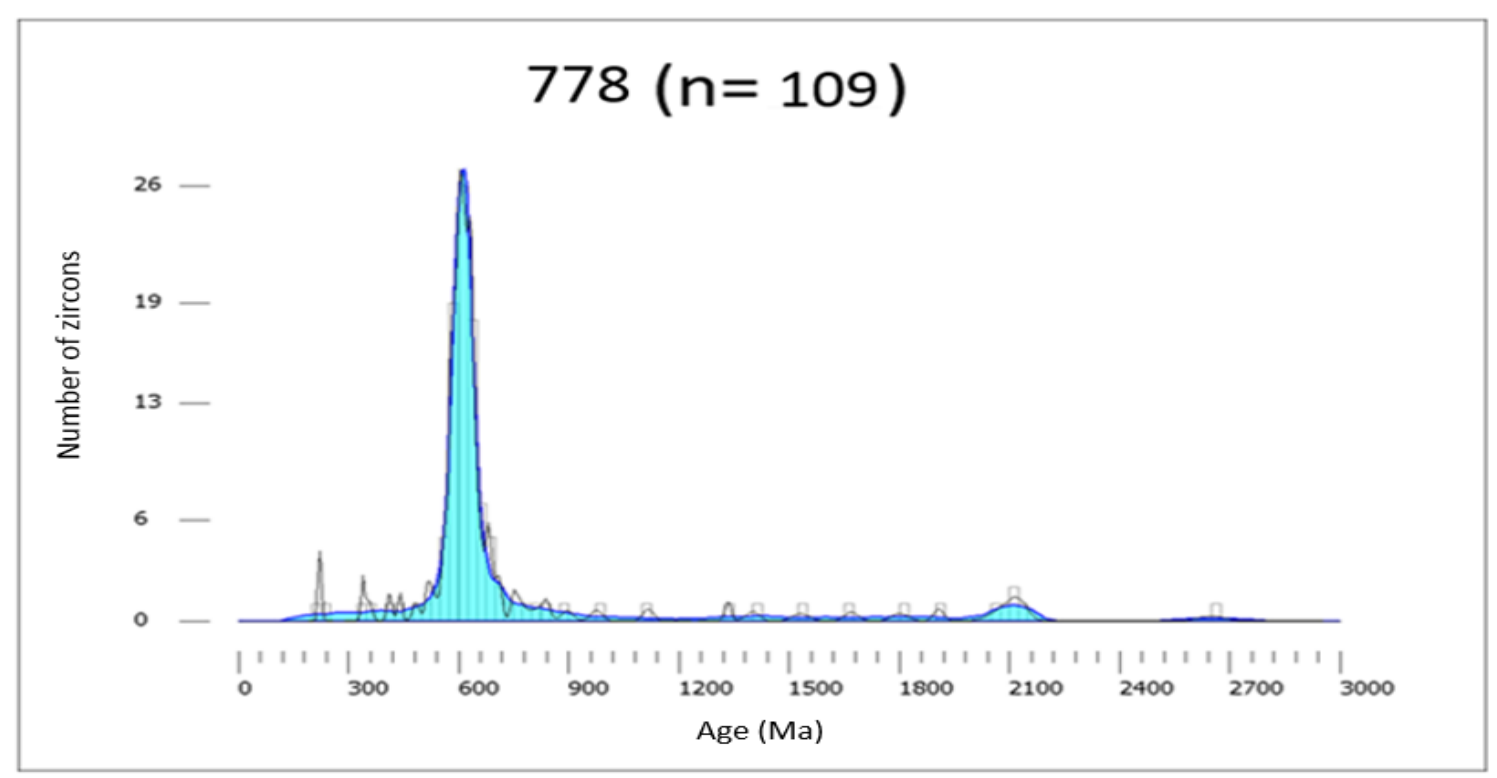

Figure 16. PDP (black line) and KDE (blue) from sample 3578. The $X$ axis is age (Ma), and the Y axis is number of grains. 
Sample 15066- Triassic-Jurassic (?) Wood River

Sample 15066 was collected from well 10566 (permit number 407) from Lee county, Florida. It was sampled from cuttings from 15,547-15,660 feet from the Wood River Formation ( 68

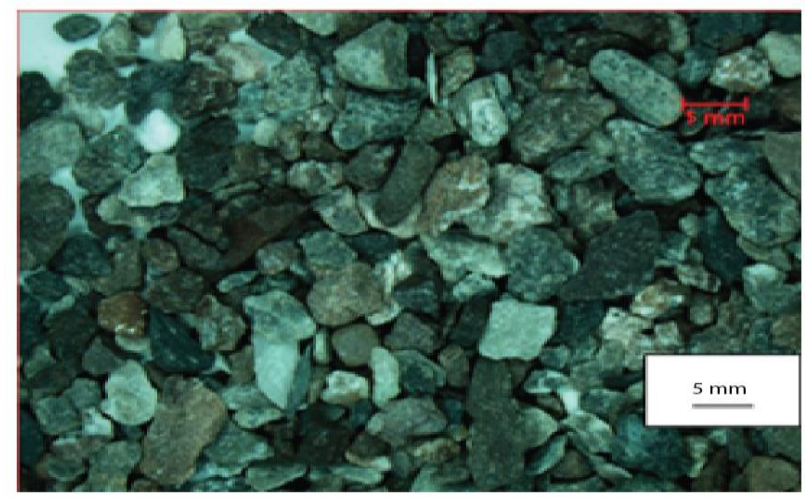

grams), which consists predominantly of red, coarse-grained sandstone and shale, which grades up into a limestone/dolomite and anhydrite (Fig. 17). In all, 46 zircons were analyzed. Three were removed for discordance and high uncertainty (Fig. 18). An age of $108 \mathrm{Ma}$ with low uncertainty (+/- 2

Figure 17. Sample 10566, collected from cuttings, is composed mainly of coarse grained shale, sandstone, and limestone.
Ma) was considered questionable based on its young age and the age of the Wood River formation, but uncertainty was low. One major age populations range from 494 to $673 \mathrm{Ma}(79 \%)$, with two peaks at

$538 \mathrm{Ma}$ and $588 \mathrm{Ma}$. Ages range from $227+/-4$ to 2,266 +/-18 Ma.

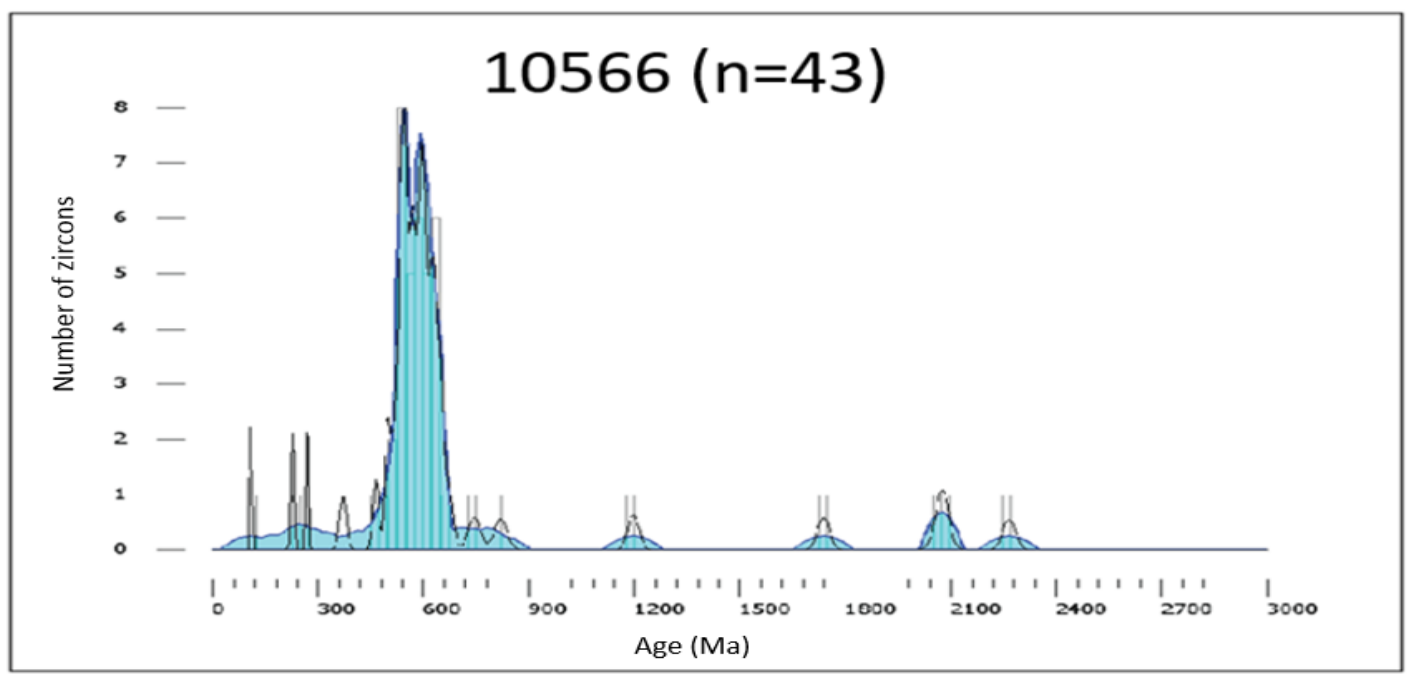

Figure 18. PDP (black line) and KDE (blue) from sample 10566. The $X$ axis is age (Ma) and the $Y$ axis is the number of grains. 
Sample 2012- Triassic-Cretaceous (?)/Paleozoic Clastic deposits

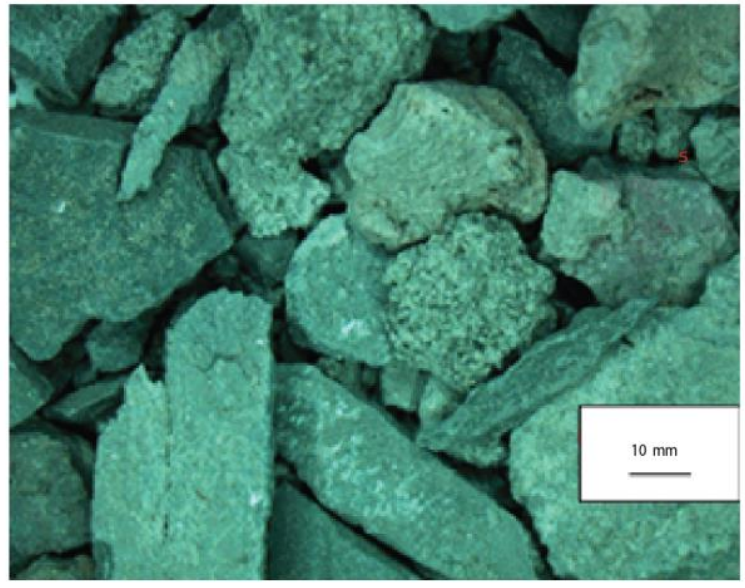

Figure 19. Sample 2012, collected from cuttings, is composed mainly of coarse grained shale and sandstone.

Sample 2012 was collected from Well 2012, Permit Number 105 from Levy county Florida within the Suwannee Basin. The sample was collected from cuttings from 4,230-4,370 feet for a total of $\sim 120$ grams. The cuttings consist primarily of coarsegrained sandstone and siltstone (Fig. 19).

In all, 106 grains were analyzed from Sample 2012. Two grains were eliminated due to high discordance. The distribution shows two age populations. The largest ranges from 465-718 Ma (52\%), with peaks at $310 \mathrm{Ma}$ and $544 \mathrm{Ma}$. A smaller population ranges from 2,000-2,306 Ma (17\%) and shows a peak at 2,132 Ma.

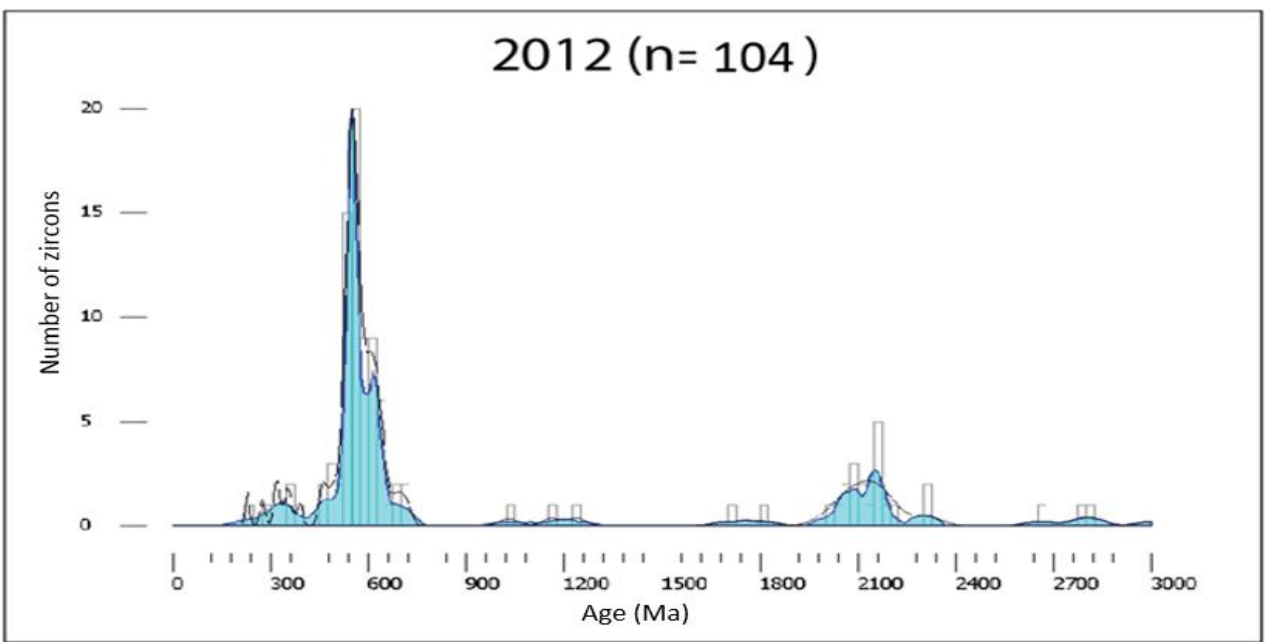

Figure 20. PDP (black line) and KDE (blue) from sample 2012. The $X$ axis is age (Ma), and the $Y$ axis is number of grains. 
Sample ODP-Offshore south Florida phylite.

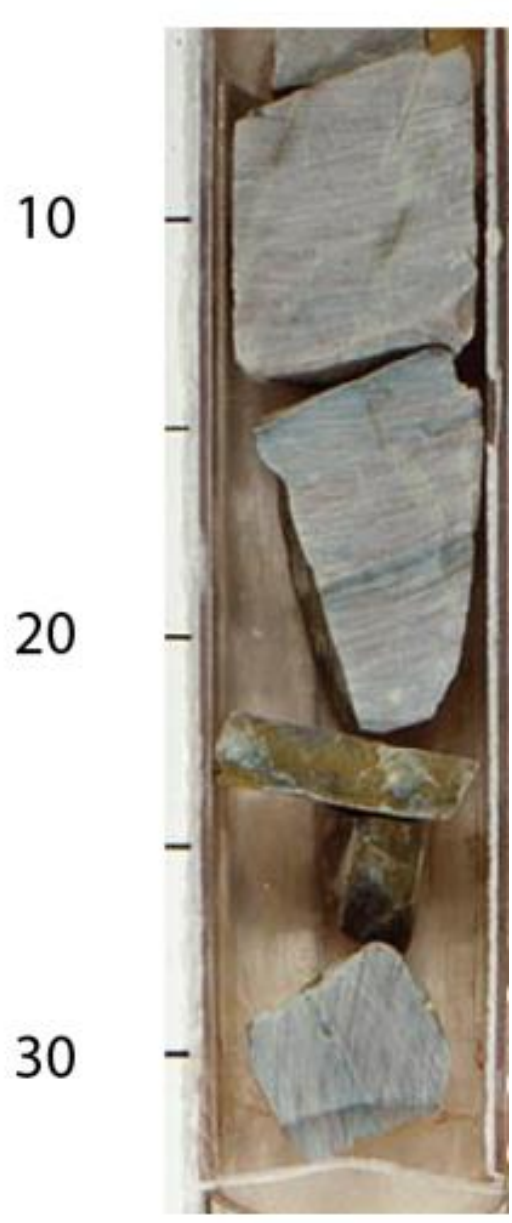
was collected, but no zircons were recovered. The arkosic

ODP was collected from ODP Expedition 77, site 0537, core 16, interval 54.57-57 (Fig. 21). Core interpretations were presented in the Deep Sea Drilling Initial Reports Volume 77 (Buffler et al., 1984). The sample was collected from around 210.5 meters below the seafloor on a small knoll north of the Campeche Escarpment. The knoll is interpreted as an uplifted titled fault block, based on seismic interpretation. The core penetrates through a thick succession of nanofossil ooze (Pliocene to Aptian), a Cretaceous oolitic limestone (Valanginian-Berriasian), Dolomite and arkosic sandstone (Berriasian), nonmarine (?) arkosic sandstone (Berriasian) and phylite (Cambrian). A sample from the Cretaceous arkosic sandstone section
Figure 21. Sample collected from interval 22-24.5, composed of phylite. Core photo provided from ODP online database. sandstone deposits are interpreted as a transgressive sequence that grades from clastic alluvial sandstones

to nearshore marine sandstones, to dolomite. The sandstone overlies pre-Mesozoic crystalline basin, which consists of foliated, metamorphic phylite (Fig. 22). Zircon grains were recovered from the metamorphic phylite section.

In all, 99 grains were analyzed. One age was eliminated for discordance. All ages range from 473 +/- 14 Ma to 756 +/- $23 \mathrm{Ma}$ (Fig. 22) and form one peak at $~ 590 \mathrm{Ma}$. 


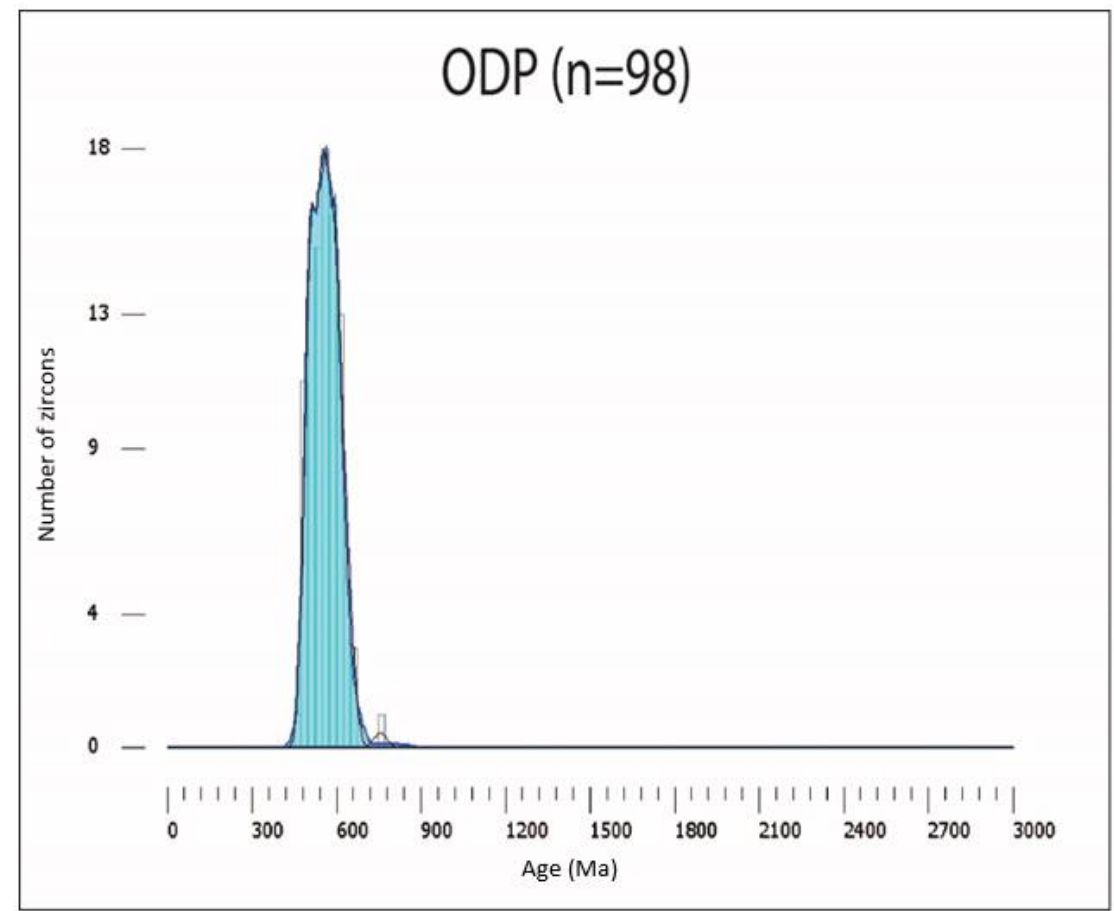

Figure 22. PDP (black line) and KDE (blue) for sample ODP. The $x$ axis is age (Ma) and the $Y$ axis is number of grains.

\section{South Florida Detrital Zircon Composite}

A composite PDP plot of only detrital zircon ages from South Florida sedimentary samples shows two main age populations (Fig. 23). A 400-700 Ma (81\% of sample) population with a prominant peak at $605 \mathrm{Ma}$, and a smaller 2,000-2,200 Ma population (6.8\% of sample) with a peak around 2,080 Ma. A plot comparing distributions of individual South Florida samples shows the samples all share one predominant age population between 580-610 Ma for all samples (Fig. 24; Fig. 25). Common age peaks were identified if they were present in four or more samples. Populations common to multiple South Florida samples include 180-450 Ma, 500620 Ma, 630-900 Ma, 1,900-2,300 Ma (Fig. 26). 


\section{SFB all samples $(n=535)$}

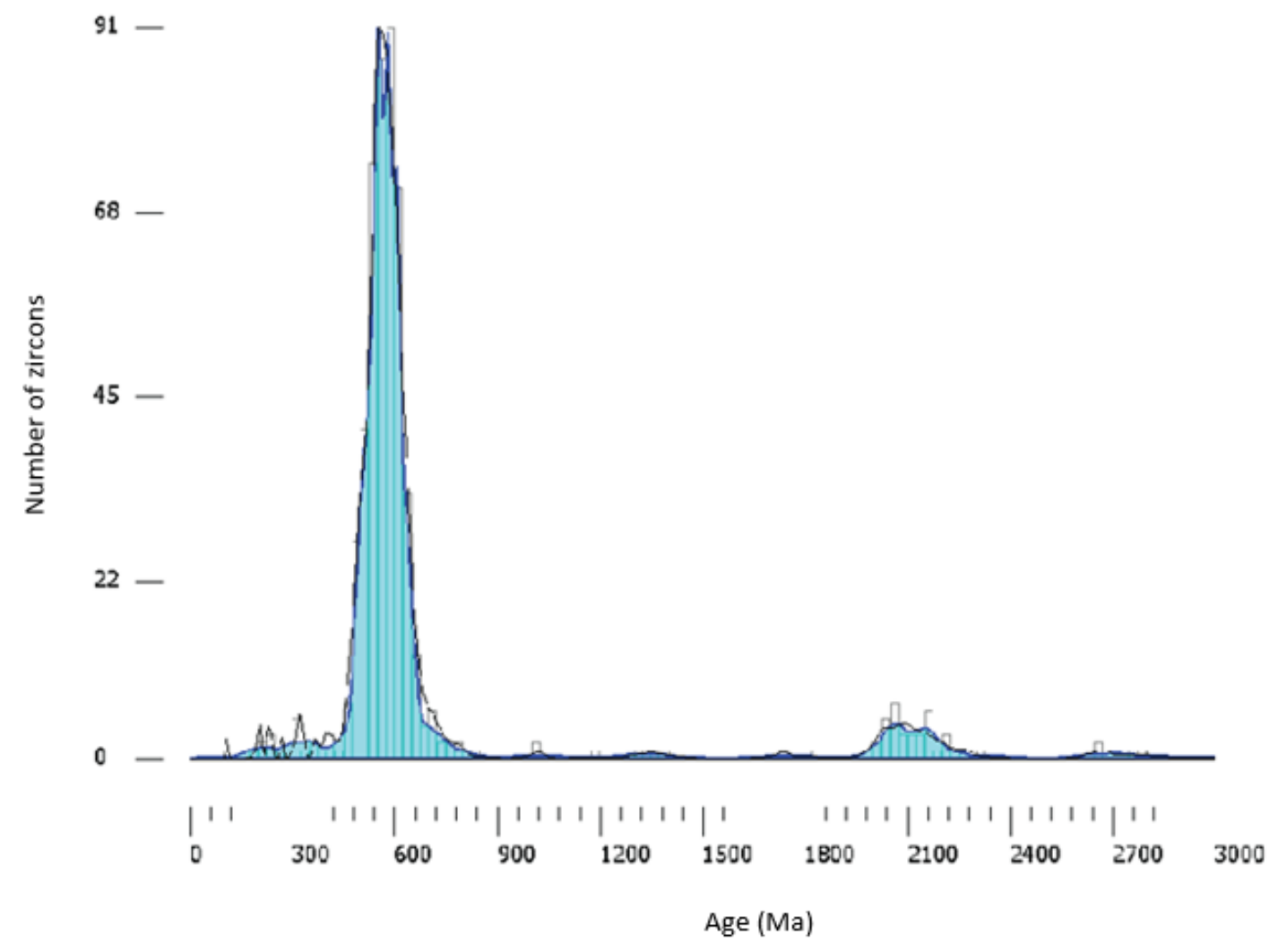

Figure 23. Cumulative PDP and KDE for the SFB (excluding sample ODP). The $X$ axis is age (Ma), and the $Y$ axis is number of grains. The two predominant peaks are $605 \mathrm{Ma}$ and $2080 \mathrm{Ma}$. 


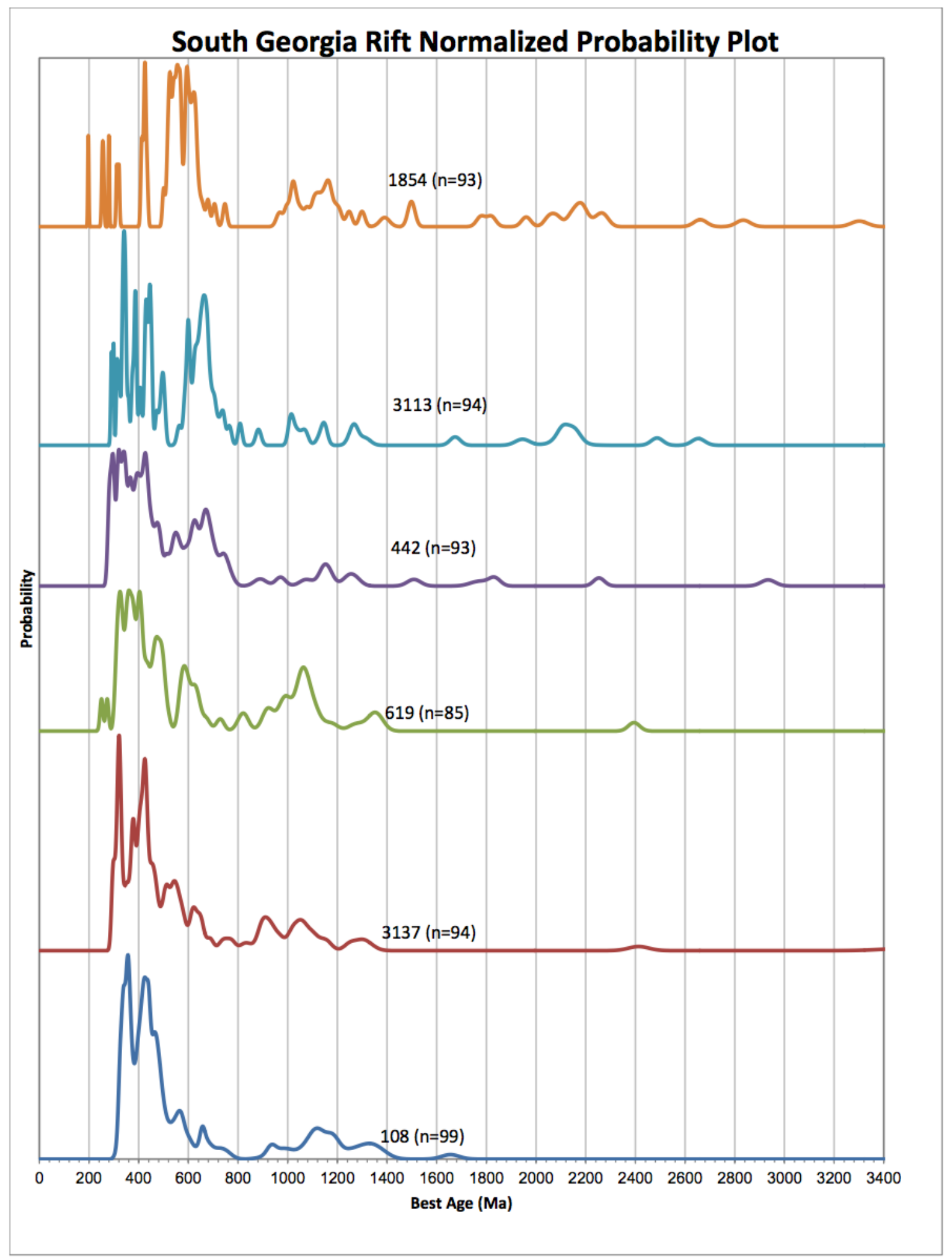

Figure 24. Normalized Probability Plot for all samples in South Florida, from north to south. Largest populations range from 580 -610 Ma for all samples. 


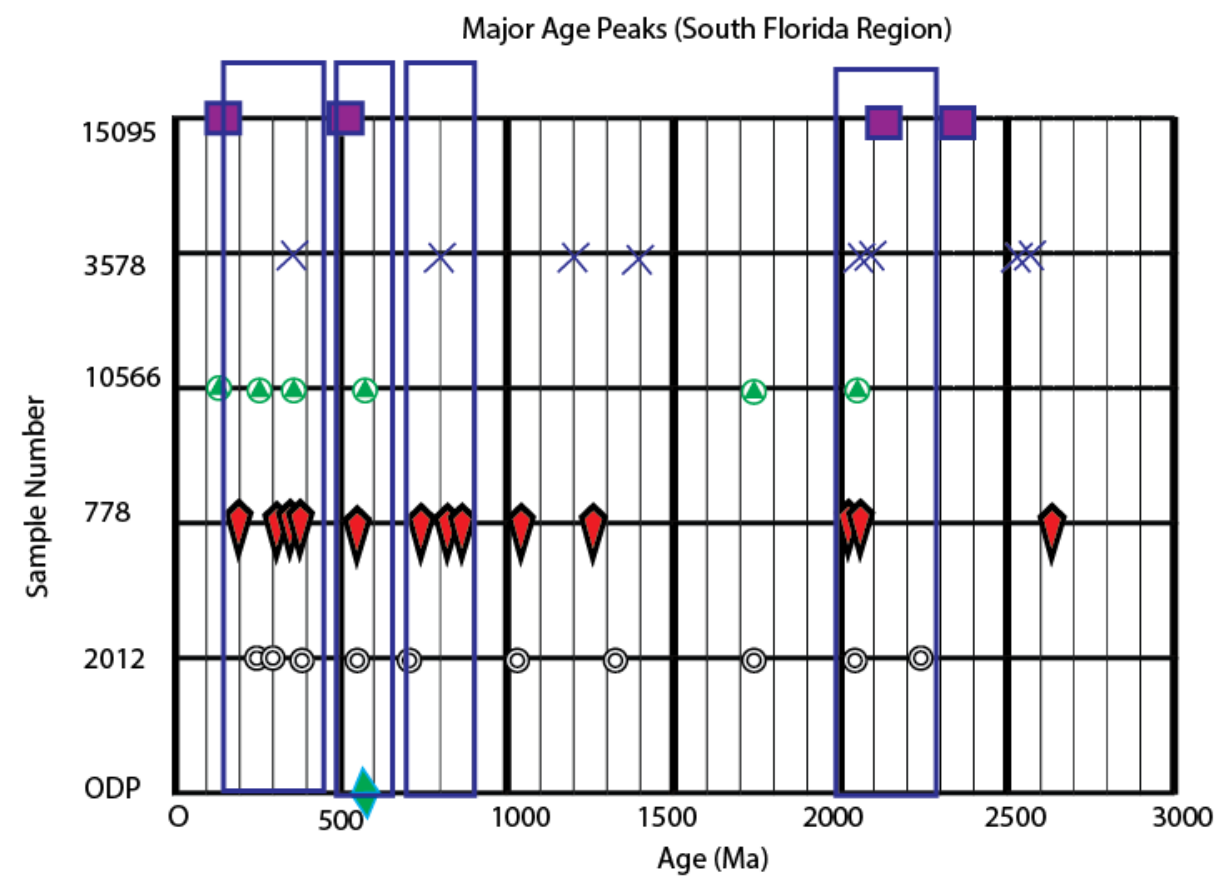

Figure 25. All age peaks for the SFB samples. Boxes correspond to major age populations. Major populations are 240-450 Ma, 500-650 Ma, 700-900 Ma and 2000-2300 Ma.

Major Age Groupings

\begin{tabular}{|c|c|}
\hline Major Age Groups (South Florida) & Sample \\
\hline $250-450 \mathrm{Ma}$ & $\begin{array}{l}15095 \text { (0 zircons } 0 \%), 3578 \text { ( } 1 \text { zircon, } 0.9 \%) \text {, } \\
10566 \text { ( } 2 \text { zircons, } 4.5 \%), 778 \text { (4 zircons, } \\
3.6 \%), 2012(7 \text { zircons, } 8.0 \%), \text { ODP }(0 \\
\text { zircons, } 0 \%)\end{array}$ \\
\hline $500-650 \mathrm{Ma}$ & $\begin{array}{l}15095 \text { ( } 72 \text { zircons, } 81.8 \%), 3578 \text { (89 zircons, } \\
80.1 \%), 10566 \text { (32 zircons, } 72.8 \%), 778(80 \\
\text { zircons, } 71.4 \%), 2012(47 \text { zircons, } 53.4 \%) \text {, } \\
\text { ODP ( } 82 \text { zircons, } 77.6 \%)\end{array}$ \\
\hline $700-900 \mathrm{Ma}$ & $\begin{array}{l}15095 \text { (0 zircons, } 0 \%), 3578 \text { (4 zircons, } \\
3.6 \%), 10566 \text { (0 zircons, } 0 \%), 778 \text { (6 zircons, } \\
5.4 \%), 2012 \text { ( } 2 \text { zircons, } 2.2 \%) \text {, ODP ( } 1 \text { zircon, } \\
1.02 \%)\end{array}$ \\
\hline $2000-2300 \mathrm{Ma}$ & $\begin{array}{l}15095 \text { (1 zircon, } 1.1 \%), 3578 \text { (6 zircons, } \\
5.4 \%), 10566 \text { ( } 3 \text { zircons, } 6.8 \%), 778(8 \\
\text { zircons, } 7.1 \%), 2012(16 \text { zircons, } 18.2 \%) \text {, } \\
\text { ODP (0 zircons, } 0 \%)\end{array}$ \\
\hline
\end{tabular}

Table 5. Table of major age groups within the SFB samples, and their relative proportions. 
Evaluation of U/Th ratios in South Florida zircon reveals two age populations of metamorphic zircon were present in all samples: 400-800 Ma, and 1900-2300 Ma (Fig. 26). The percentage of these populations represented in each sample are listed in Figure 27 and Table 6. A population of 400-800 Ma metamorphic zircon is the most abundant within the SFB.

Metamorphic zircon make up $28 \%$ of sample 3578 , and $>10 \%$ of samples $15095,10566,778$, and 2012. A less abundant population of 1900-2300 Ma zircon grains form up to $6.9 \%$ of a single sample (sample 2012). 

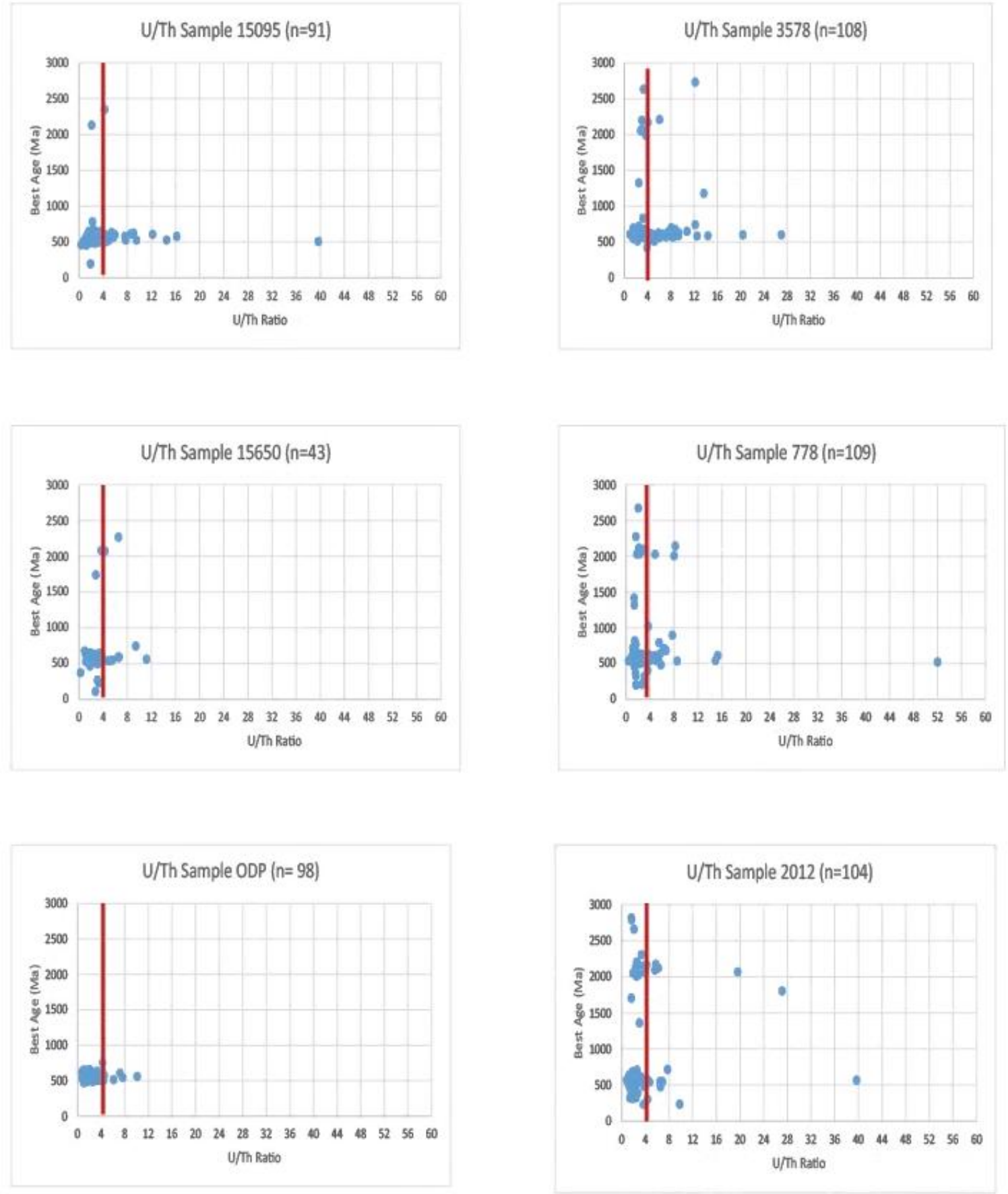

Figure 26. Ages and U/Th ratios for detrital zircon of the South Florida samples. Metamorphic zircons were identified based on a U/Th ratio greater than 5 . 


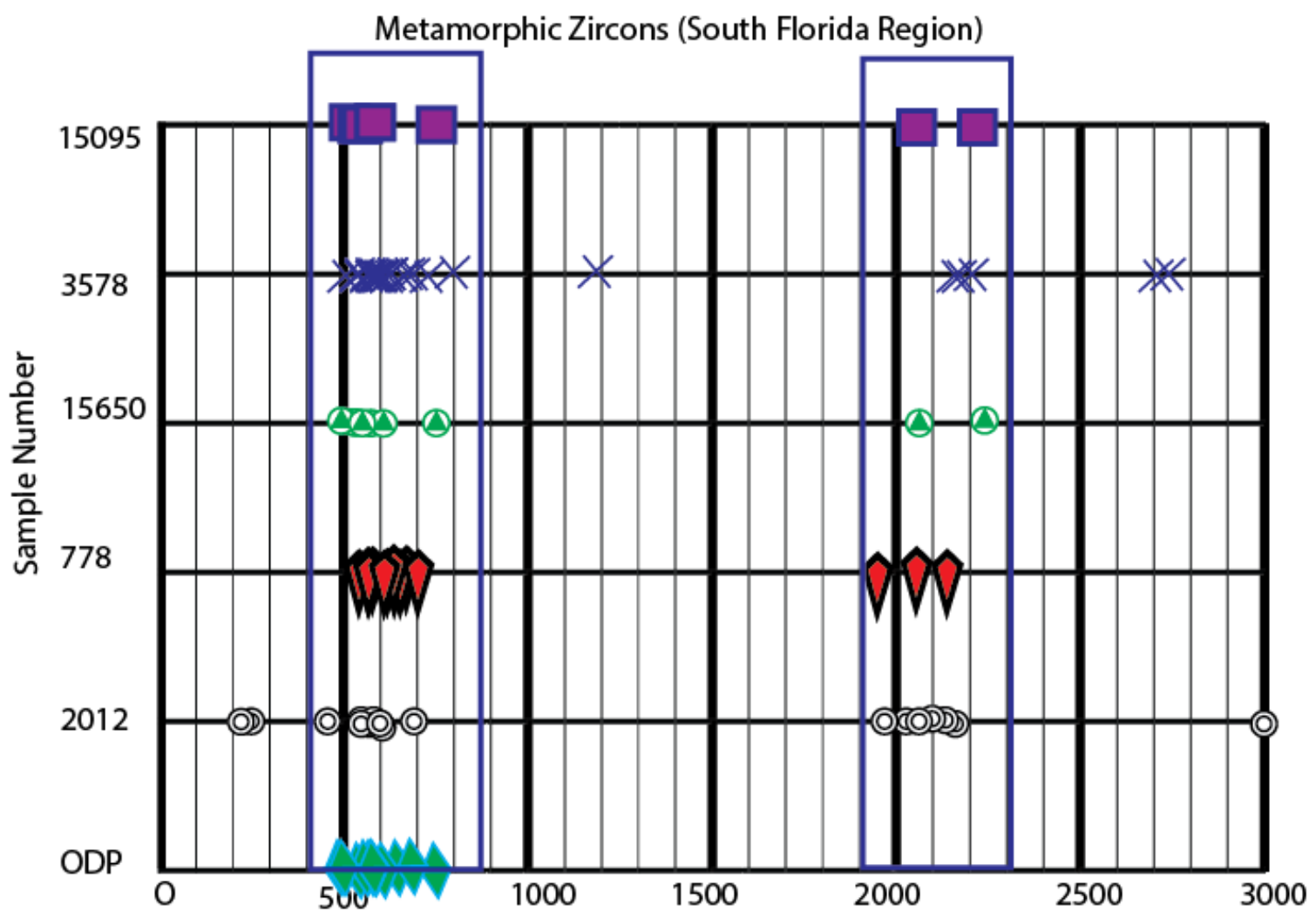

Age (Ma)

Figure 27. Major age peaks for metamorphic detrital zircon in the SFB (blue boxes). Two groupings are present between $400-800$ Ma and 1900-2300 Ma.

\section{Metamoprhic Zircons}

\begin{tabular}{|l|l|}
\hline $\begin{array}{l}\text { Age Group Metamorphic Zircons (South } \\
\text { Florida) }\end{array}$ & Sample \\
\hline $400-800 \mathrm{Ma}$ & $15095(14$ zircons, 15.9\%), 3578 (31 zircons, \\
& $\begin{array}{l}28 \%) 10566(5 \text { zircons, 11.4\%), 778 (13 } \\
\text { zircons, 12\%), 2012 (4 zircons, 10.2\%) ODP } \\
(4 \text { zircons, 4.0\% })\end{array}$ \\
\hline $1900-2300 \mathrm{Ma}$ & $\begin{array}{l}15095(0 \text { zircons, 0\%), 3578 (2 zircons, } \\
1.8 \%), 10566(1 \text { zircons, 2.2\%), 778 (2 } \\
\text { zircons, 1.8\%), 2012 (5 zircons, (6.9\%), ODP } \\
(0 \text { zircons, 0\%) }\end{array}$ \\
\hline
\end{tabular}

Table 6. Major metamorphic zircon age groupings identified in the SFB. Major age groups present are 400-800 Ma and 1900$2300 \mathrm{Ma}$. 


\section{South Georgia Rift Detrital Zircon Ages}

A map of samples collected from Southern Georgia is displayed in Figure 28. Samples

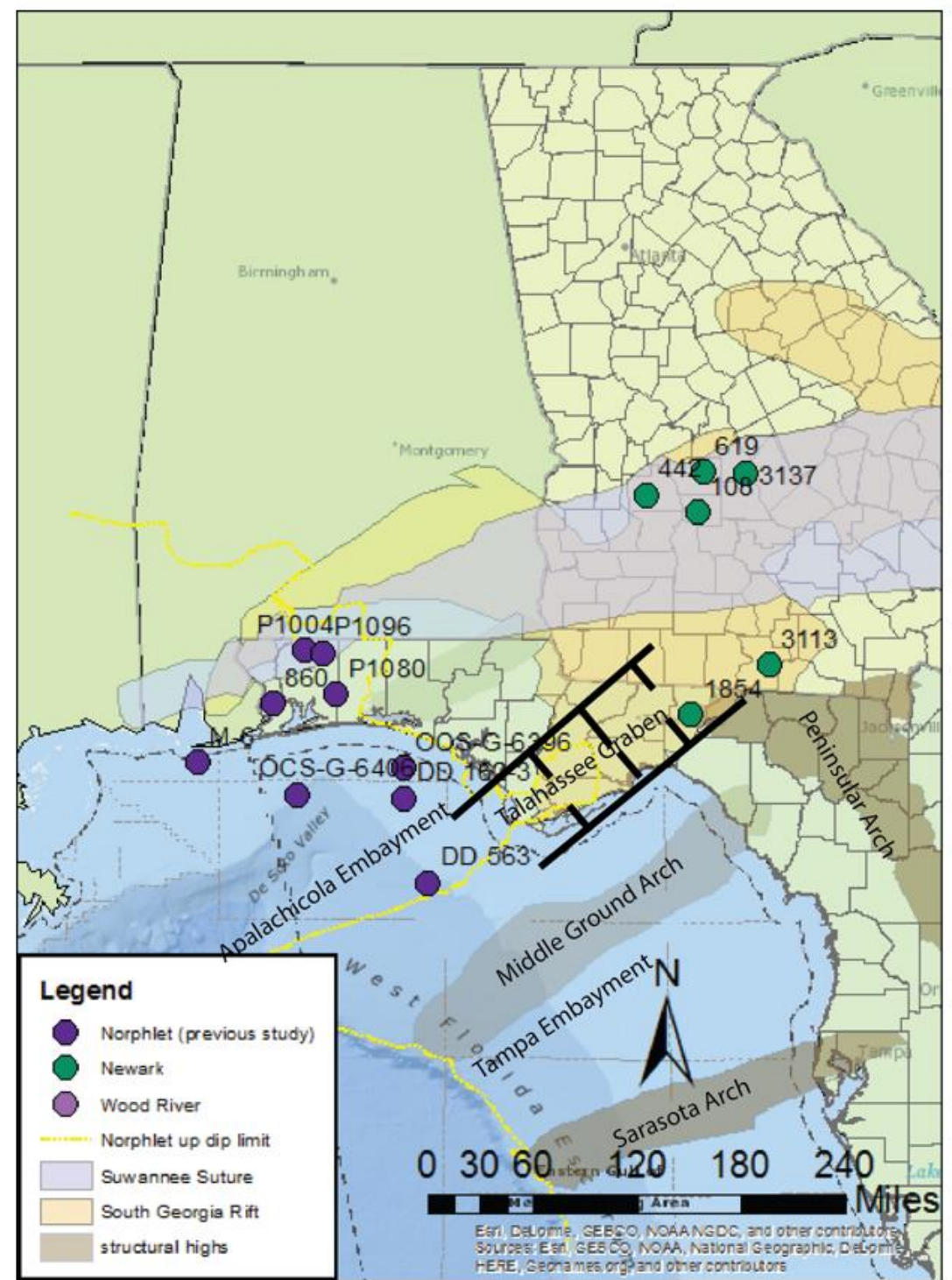

collected for detrital

zircon analysis from

the South Georgia

rift include samples

442, 619, 108, 3137,

and 3113. Sample

1854 was collected

from the Florida

Geological Survey

from the

Tallahassee Graben,

which is likely an

extension of the

South Georgia Rift.

Figure 28. Location of the Newark deposits (green) sampled in this study. Other samples localites are Norphlet samples (blue) from Lovell (2010) and Lisi (2013). 
Sample 108 was collected from well GGS number 108, Crisp county, Georgia form 4820-5010 feet from drill cuttings for a total sample mass

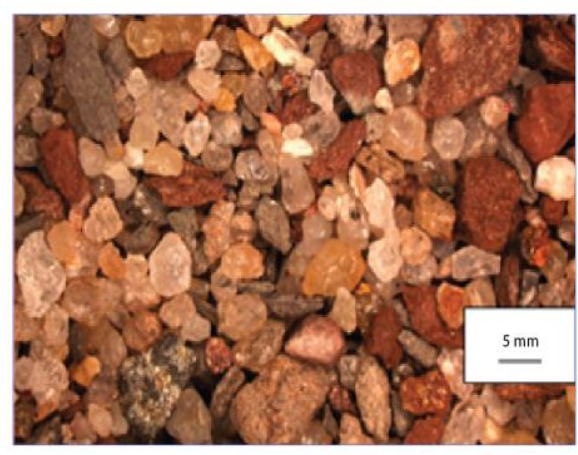
of $\sim 340$ grams. The lithology consists primarily of coarsegrained sandstone (Fig. 29).

In all, 121 zircons were dated for this study. Twenty two ages were eliminated that had high uncertainty and/or high discordance. Ages range from $323+/-7$ Ma to 1655

Figure 29. Sample 108, collected from cuttings, composed +/- $35 \mathrm{Ma}$ (Fig. 30). Age populations are mainly of quartz, barite, and sandstone fragments. 307 to $376 \mathrm{Ma}(24 \%), 384$ to $507 \mathrm{Ma}(40 \%)$, 530 to $590 \mathrm{Ma}$ (7.5\%), 630 to $680 \mathrm{Ma}(6.5 \%)$, and $900 \mathrm{Ma}$ to $1400 \mathrm{Ma}(26 \%)$. Major peak within these populations occur at are $329 \mathrm{Ma}, 440 \mathrm{Ma}, 554 \mathrm{Ma}, 667 \mathrm{Ma}, 1110 \mathrm{Ma}$, and $1640 \mathrm{Ma}$.

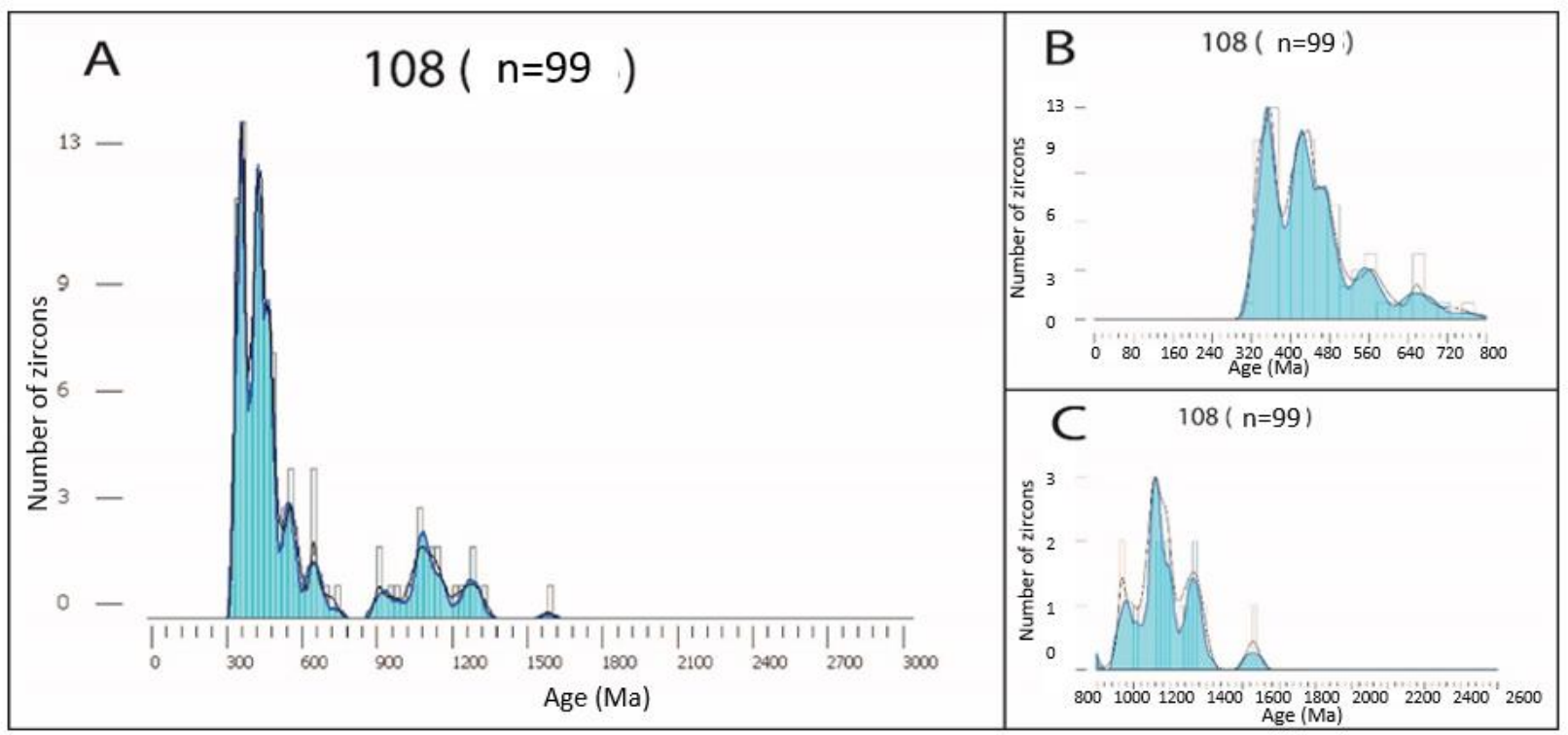

Figure 30. PDP (black line) and KDE (blue) from sample 108. The $X$ axis is age (Ma), and the $Y$ axis is number of grains. A: Detrital zircons from 0 to $3000 \mathrm{Ma}$. B: Detrital zircons from 0 to $800 \mathrm{Ma}$. C: Detrital zircons from 800 to $3000 \mathrm{Ma}$. 
Sample 3113 was collected from well GGS number 3113, Lowndes county, Georgia. The

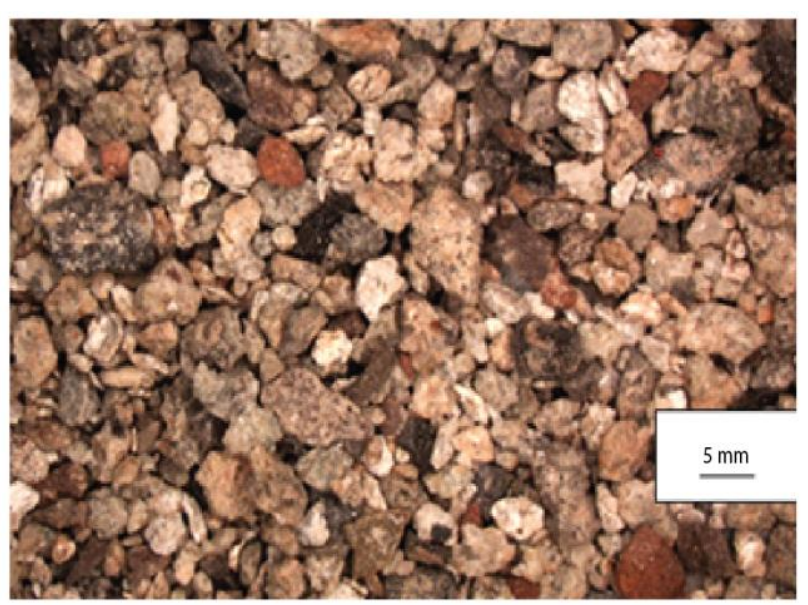

sample was collected from $8130-8510$ feet

from cuttings yielding a sample mass of $\sim 420$

grams. Lithology varies from medium-grained

sandstone to red and black shale (Fig. 31).

In all, 98 zircons were analyzed, of

which 4 were removed due to discordance.

Figure 31. Sample 3113, collected from cuttings and composed mainly of quartz, shale.

Ages range from $291+/-3$ Ma to 2653

+/- $17 \mathrm{Ma}$ (Fig. 32). Age populations

include 290 to $450 \mathrm{Ma}(40.4 \%), 560$ to $741 \mathrm{Ma}(30.9 \%), 989$ to $1296 \mathrm{Ma}$ (10.6\%), and 2006 to

$2158 \mathrm{Ma}(8.5 \%)$. Peak ages are $290 \mathrm{Ma}, 339 \mathrm{Ma}, 444 \mathrm{Ma}, 600 \mathrm{Ma}$, and $656 \mathrm{Ma}$.

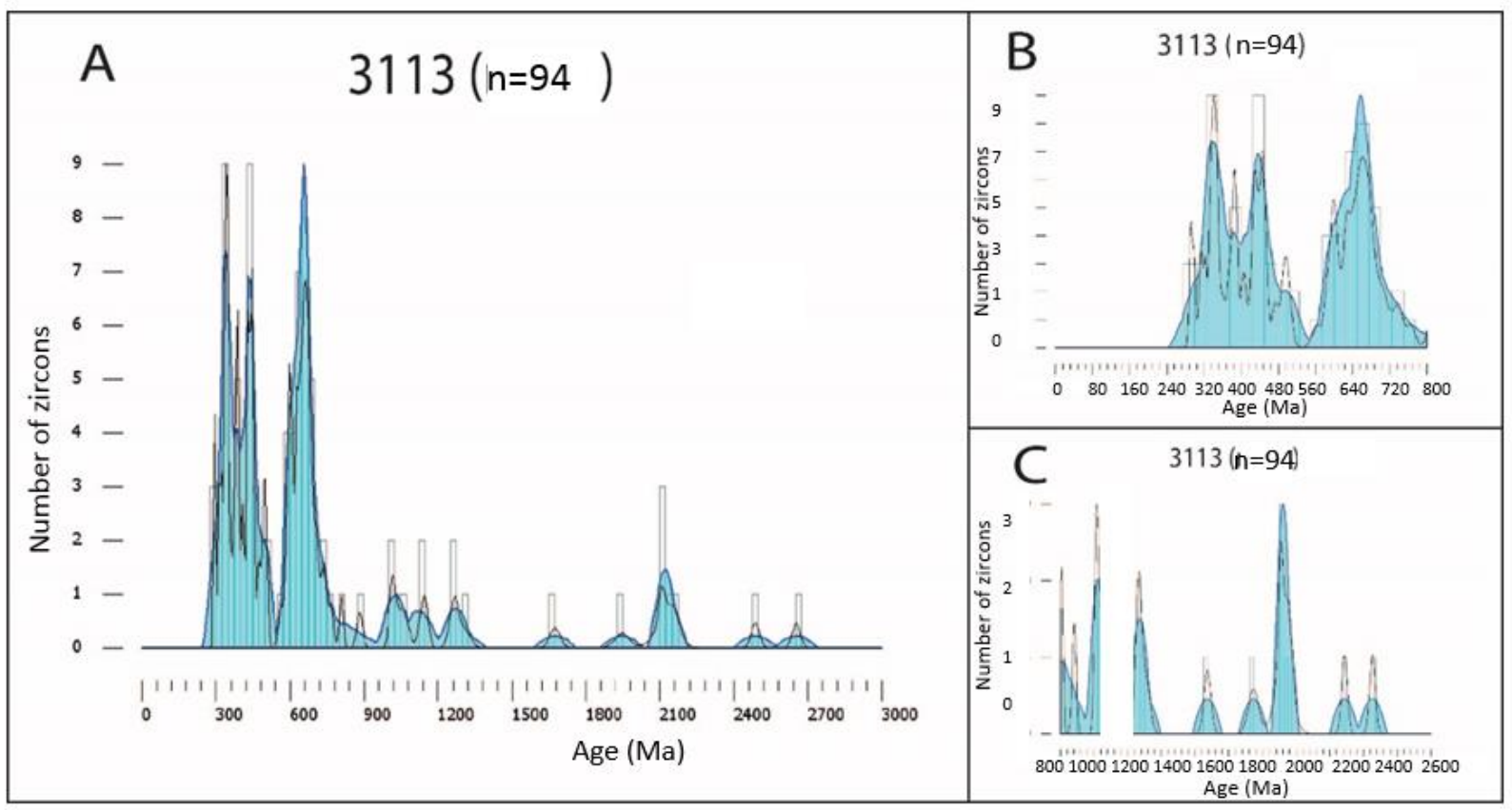

Figure 32. PDP (black line) and KDE (blue) from sample 3113. The $X$ axis is age (Ma), and the $Y$ axis is number of grains. A: Detrital zircons from 0 to $3000 \mathrm{Ma}$. B: Detrital zircons from 0 to $800 \mathrm{Ma}$. C: Detrital zircons from 800 to $3000 \mathrm{Ma}$. 
Sample 619 was sampled from well GGS

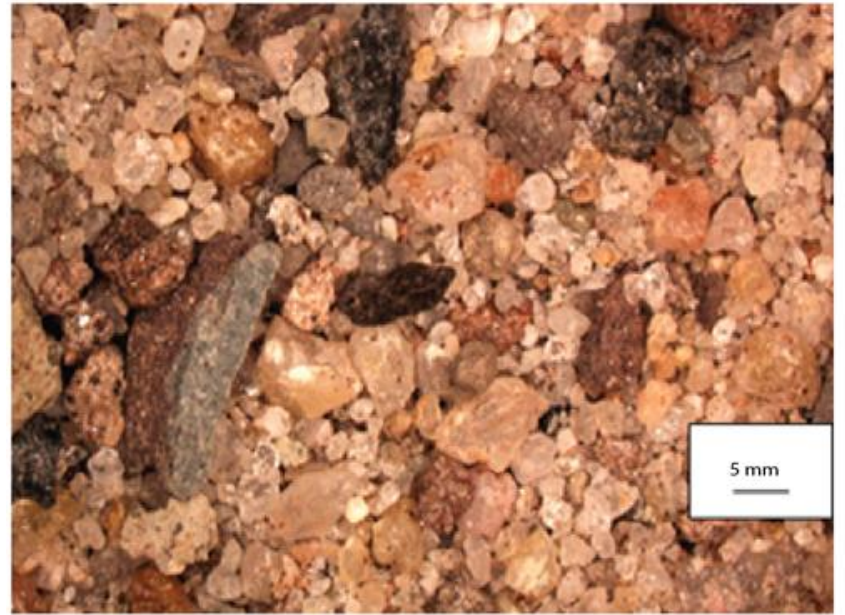

Figure 33. Sample 619. Collected from cuttings, and composed mainly of barite, quartz, shale and sandstone. number 619, Dooly county, Georgia. The sample was collected from 3,510-3,750 feet depth from cuttings for a total sample mass of $\sim 330$ grams. Lithology is composed almost entirely of quartz sandstone (Fig. $33)$.

In total, 90 zircons were analyzed during this

study. Five were not included due to high uncertainty and/or discordance. Ages range from 250 +/- 7 to 2394 +/- $17 \mathrm{Ma}$ (Fig. 34). Major age populations include 310 to $421 \mathrm{Ma}$ (34.1\%), 456 to $503 \mathrm{Ma}(14.1 \%), 550$ to $670 \mathrm{Ma}(13.0 \%$, and 900 to $11370 \mathrm{Ma}(25.9 \%)$. Age peaks are $322 \mathrm{Ma}, 372 \mathrm{Ma}, 406 \mathrm{Ma}, 487 \mathrm{Ma}, 592 \mathrm{Ma}$, and $1058 \mathrm{Ma}$.

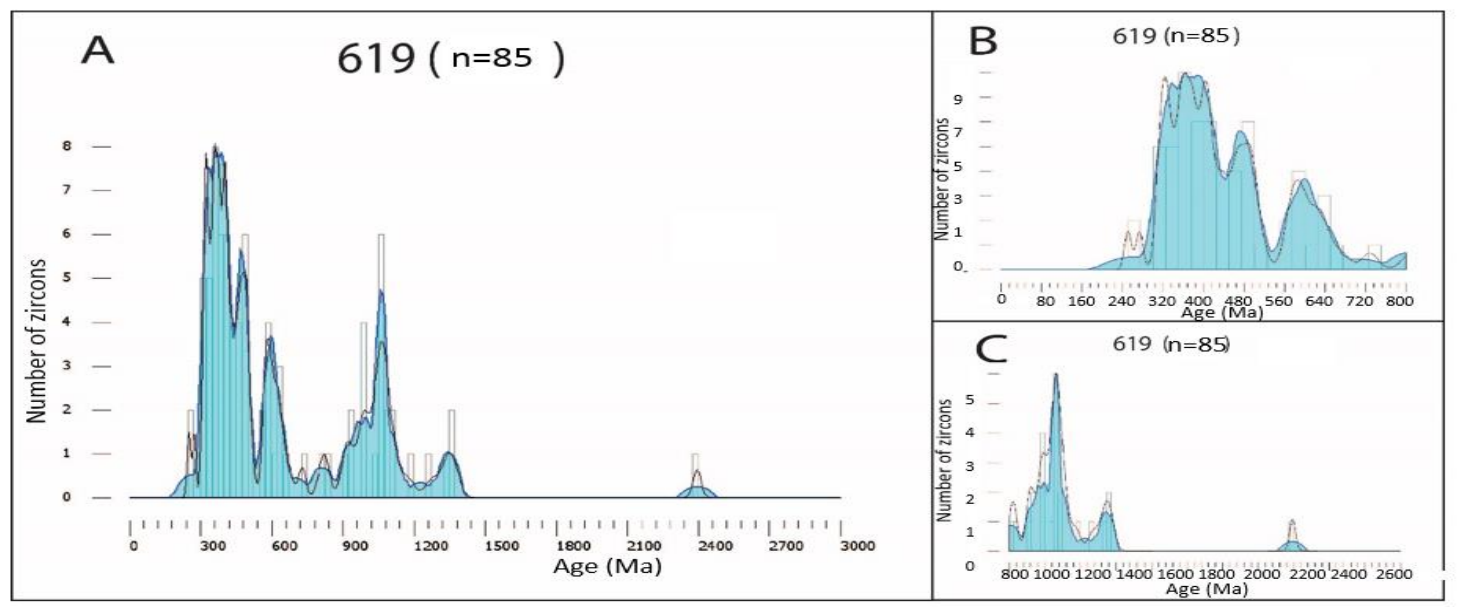

Figure 34. PDP (black line) and KDE (blue). The $X$ axis is age (Ma), and the $Y$ axis is number of grains. A: Detrital zircons from 0 to 3000 Ma. B: Detrital zircons from 0 to 800 Ma. C: Detrital zircons from 800 to 3000 Ma. 


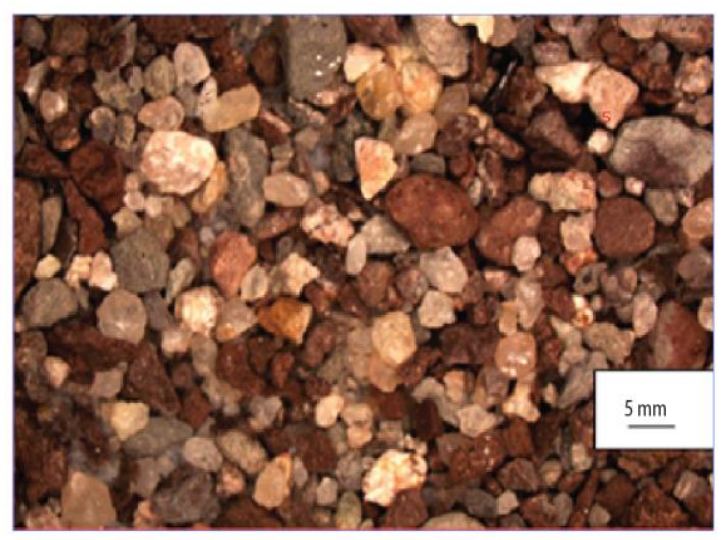

Sample 3137- South Georgia Rift Newark Group

Sample 3137 was sampled from Pulaski county,

Georgia. Sample was collected from 5320-5827 feet from cutting totaling $\sim 400$ grams. Lithology consists mostly of interbedded, medium-grained sandstone, siltstone, and shale (Fig. 35). Diabase intrusions were also common, but

Figure 35. Sample 3137 Sample was collected from cuttings, and consists mainly of red sandstone and shale, as well as barite. were not sampled in this study.

In total, 98 zircons were dated.

Four were removed for high uncertainty and/or discordance. Age ranges from 294 +/- 6 to 3470 +/- 90 Ma (Fig. 36). Major age populations include 290 to $340 \mathrm{Ma}(17 \%), 370$ to $440 \mathrm{Ma}$ (26.6\%), 490 to to $640 \mathrm{Ma}(20 \%), 870$ to $940 \mathrm{Ma}(5.3 \%)$, and 1000 to $1400 \mathrm{Ma}(11.8 \%)$. Age peaks at $320 \mathrm{Ma}, 442 \mathrm{Ma}, 533 \mathrm{Ma}, 632 \mathrm{Ma}, 902 \mathrm{Ma}$, and $1044 \mathrm{Ma}$.

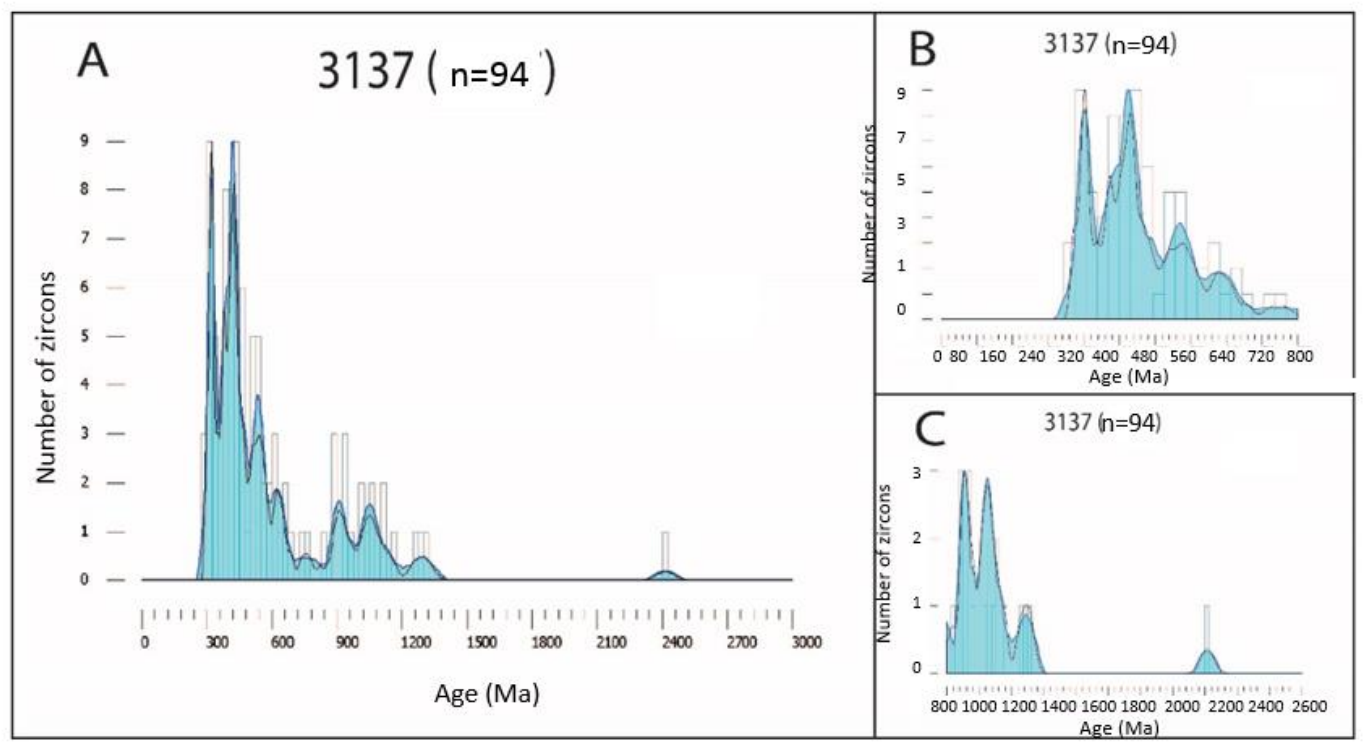

Figure 36. PDP (black line) and KDE (blue) from sample 3137. The $X$ axis is age (Ma), and the $Y$ axis is number of grains. A: Detrital zircons from 0 to $3000 \mathrm{Ma}$. B: Detrital zircons from 0 to $800 \mathrm{Ma}$. C: Detrital zircons from 800 to $3000 \mathrm{Ma}$. 
Sample 442-South Georgia Rift Newark Group

Sample 442 was collected from well GGS number 442, Sumter country, Georgia. Sample

was collected from 3980-4520 feet from cuttings and

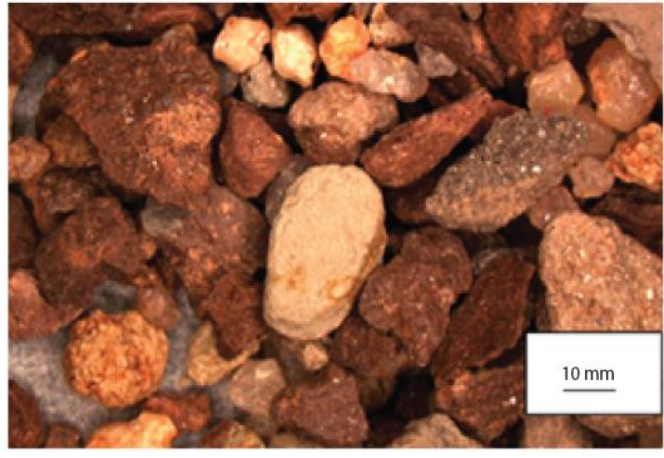

Figure 37. Sample 442. Sample was collected from cuttings/rock chips, and consists mainly of coarse grained sandstone and shale. totaled $~ 500$ grams. Lithology consists mainly of coarse-

grained red shale and coarse grained sandstone (Fig. 37).

In total, 105 zircons were analyzed for this study.

Twelve were removed due to high uncertainty and/or

discordance. Ages range from $279+/-6$ to 2934

+/- 28 Ma (Fig. 38). Major age clusters include

248-300 Ma (8.6\%), 310 to $340 \mathrm{Ma}(13.9 \%), 380$ to $450 \mathrm{Ma}(31.2 \%)$, and 598 to $750 \mathrm{Ma}$

(21.5\%). Age peaks are observed at $248 \mathrm{Ma}, 345 \mathrm{Ma}, 430 \mathrm{Ma}, 549 \mathrm{Ma}, 633 \mathrm{Ma}$, and $671 \mathrm{Ma}$.

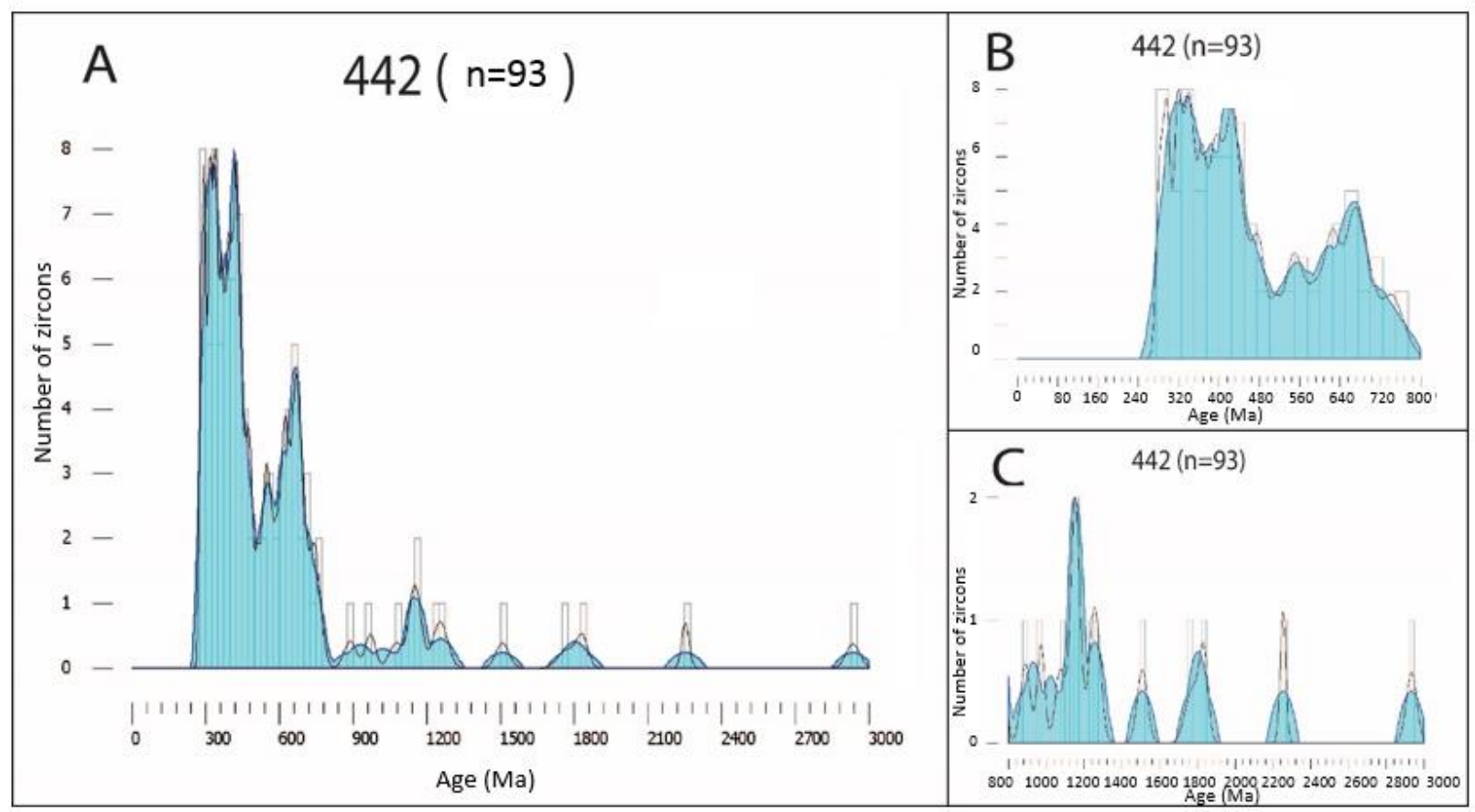

Figure 38. PDP (black line) and KDE (blue) from sample 442. The $X$ axis is age (Ma), and the $Y$ axis is number of grains $A$ : Detrital zircons from 0 to $3000 \mathrm{Ma}$. B: Detrital zircons from 0 to $800 \mathrm{Ma}$. C: Detrital zircons from 800 to $3000 \mathrm{Ma}$. 
Sample 1854- Tallahassee Graben Rift Newark (?) rift clastics

Sample 1854 was collected from the well 1854 (permit number 95) from Jefferson

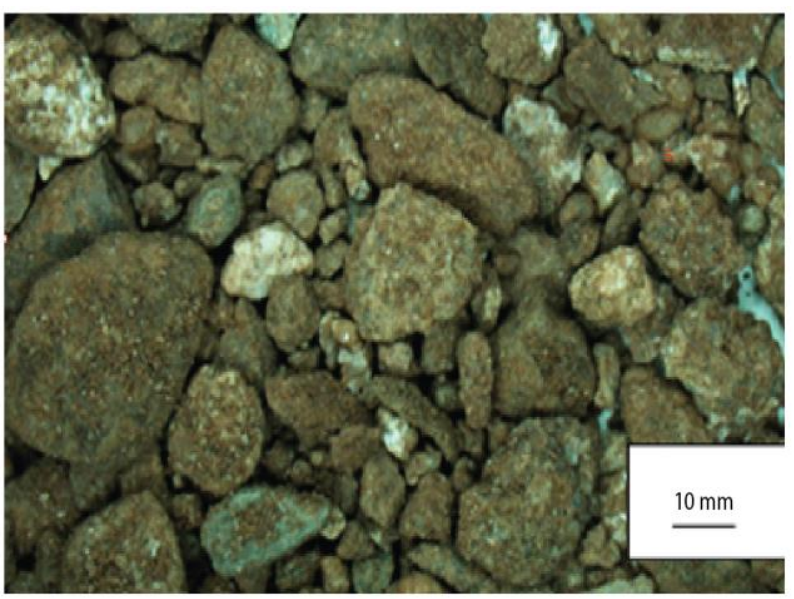

county, Florida. Sample was collected from 7280-7610

feet from cuttings and amassed $\sim 68$ grams. Lithology

consists mainly of interbedded red fine-grained (?)

sandstone and shale (Fig. 39).

Ninety four zircons were analyzed. One zircon

was excluded due to high uncertainty. Ages range from

$197+/-3$ to 3295 +/- 28 Ma (Fig. 40).

Figure 39. Sample 1854. Sample was collected from cuttings/rock chips, and consists of coarse grained sandstone and shale.

Major age populations include 390 Ma to

$440 \mathrm{Ma}(7.5 \%) 490 \mathrm{Ma}$ to $650 \mathrm{Ma}, 955$ to $1061 \mathrm{Ma}(45 \%) 1000 \mathrm{Ma}$ to $1240 \mathrm{Ma}(18 \%)$ and 2000

Ma to $2270 \mathrm{Ma}(8.6 \%$; Fig.40) Major age peaks include $424 \mathrm{Ma}, 566 \mathrm{Ma}, 596 \mathrm{Ma}, 1024 \mathrm{Ma}$, and $1161 \mathrm{Ma}$

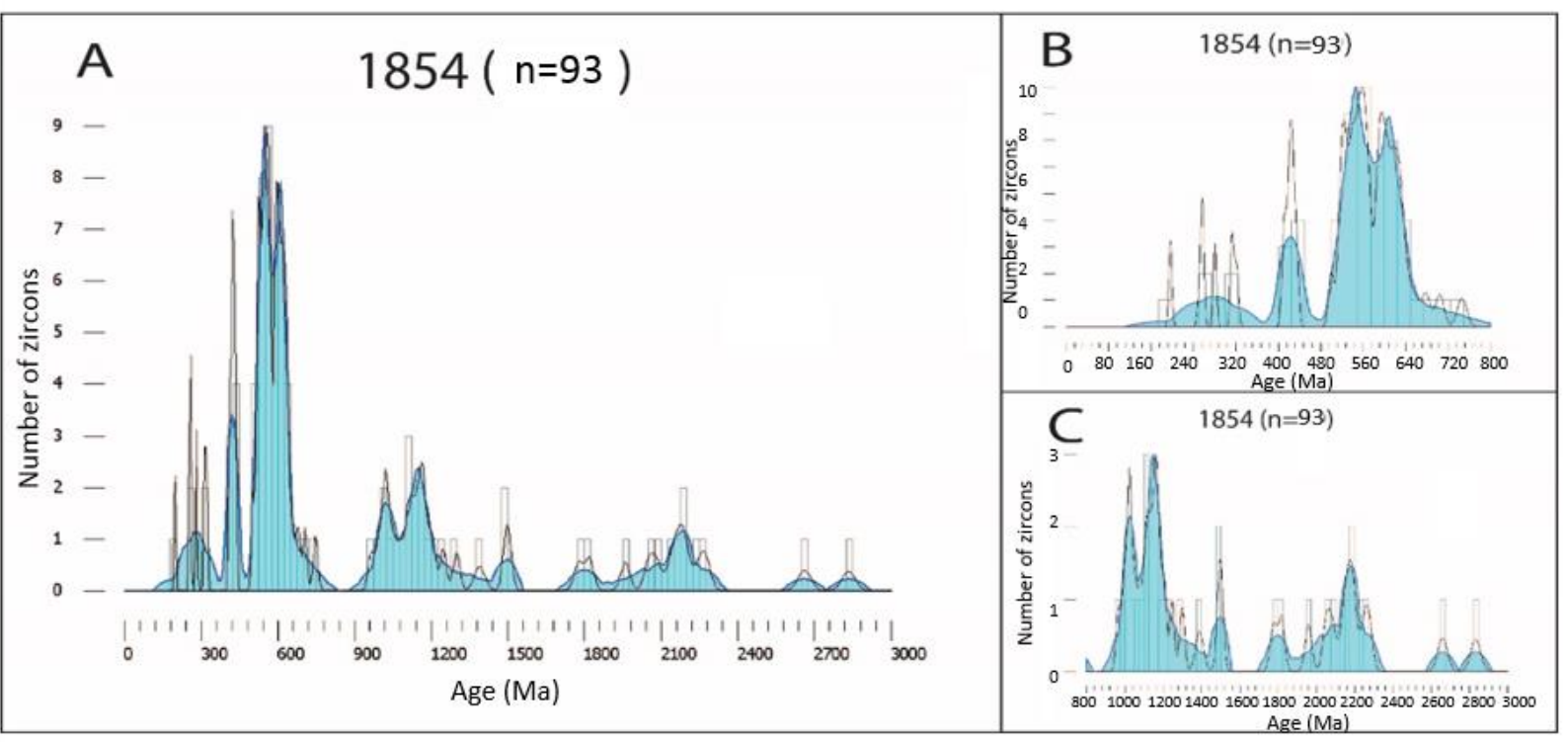

Figure 40. PDP (black line) and KDE (blue) from sample 1854. The $X$ axis is age (Ma), and the $Y$ axis is number of grains A: Detrital zircons from 0 to $3000 \mathrm{Ma}$. B: Detrital zircons from 0 to $800 \mathrm{Ma}$. C: Detrital zircons from 800 to $3000 \mathrm{Ma}$. 


\section{South Georgia Rift Composite}

A composite probability density plot of detrital zircon ages from all SGR samples shows three major popultations are present. Comparision of individual sample normalized probability plots show these ages form populations in all SGR detrital zircon age signatures: 300 to $480 \mathrm{Ma}$ (41.3\%), 500 to $672 \mathrm{Ma}(24.9 \%)$, and 1300 to $1500 \mathrm{Ma}$ (17.0\%; Figures 41 and 42). Major age peaks are listed in Figure 43 and Table 7. Samples 3137, 1854, 619, 108, and 442 have large peaks between 200-500 Ma, ranging from $13.3 \%$ to $62.9 \%$ for all samples. Samples 442, 3137, 619, and 108 all have a large population from 525 -725 Ma that makes up $12.8 \%$ to $33.0 \%$ of each sample. Samples 1854, 3137, 619, 108 all have populations between 750-1100 Ma that make up $6.7-20.9 \%$ of all samples.

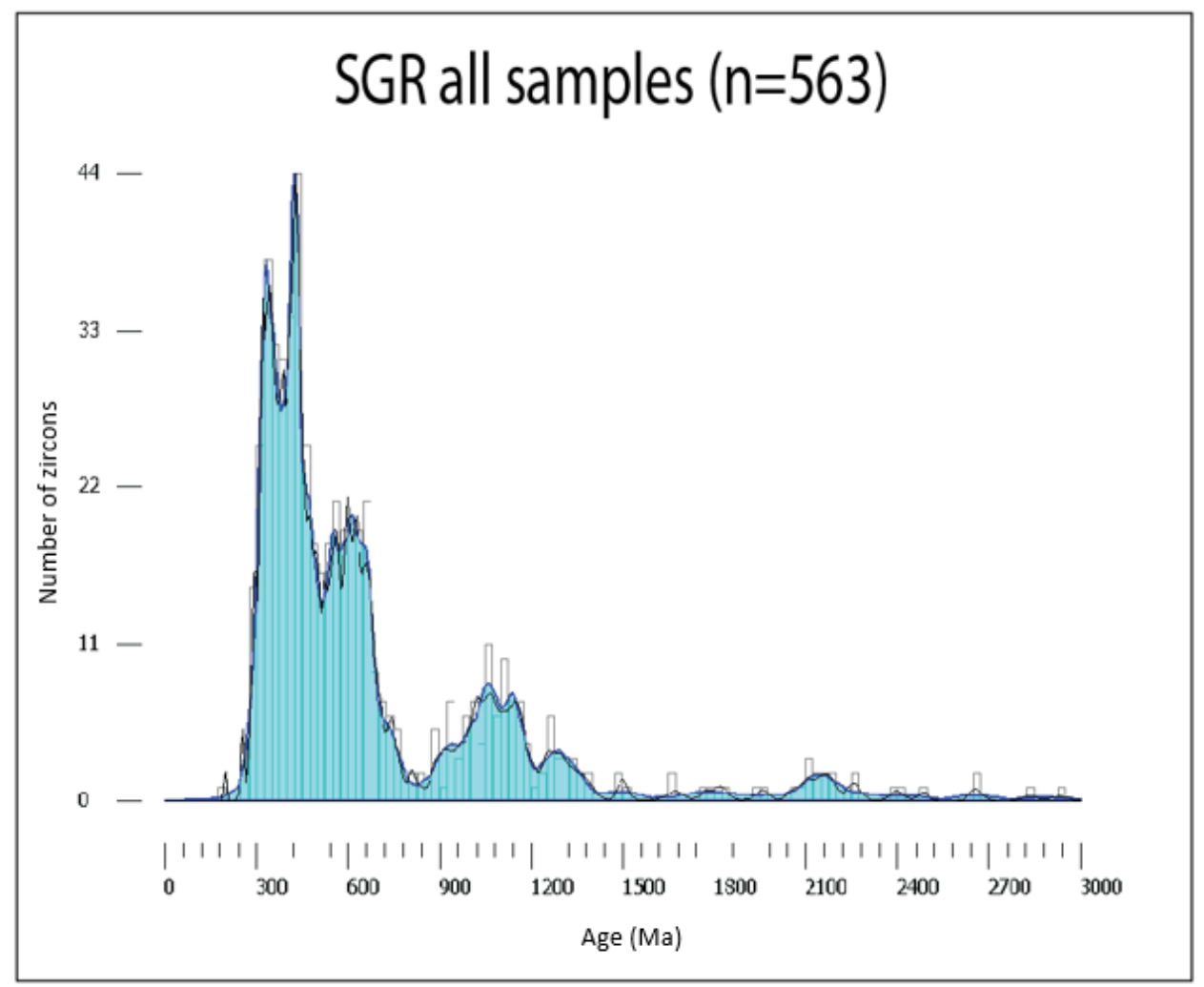

Figure 41. Cumulative PDP (black line) and KDE (blue) for all SGR samples. Major peaks include 297 to $480 \mathrm{Ma}, 509 \mathrm{Ma} 672 \mathrm{Ma}$, and 982 to $1184 \mathrm{Ma}$. 


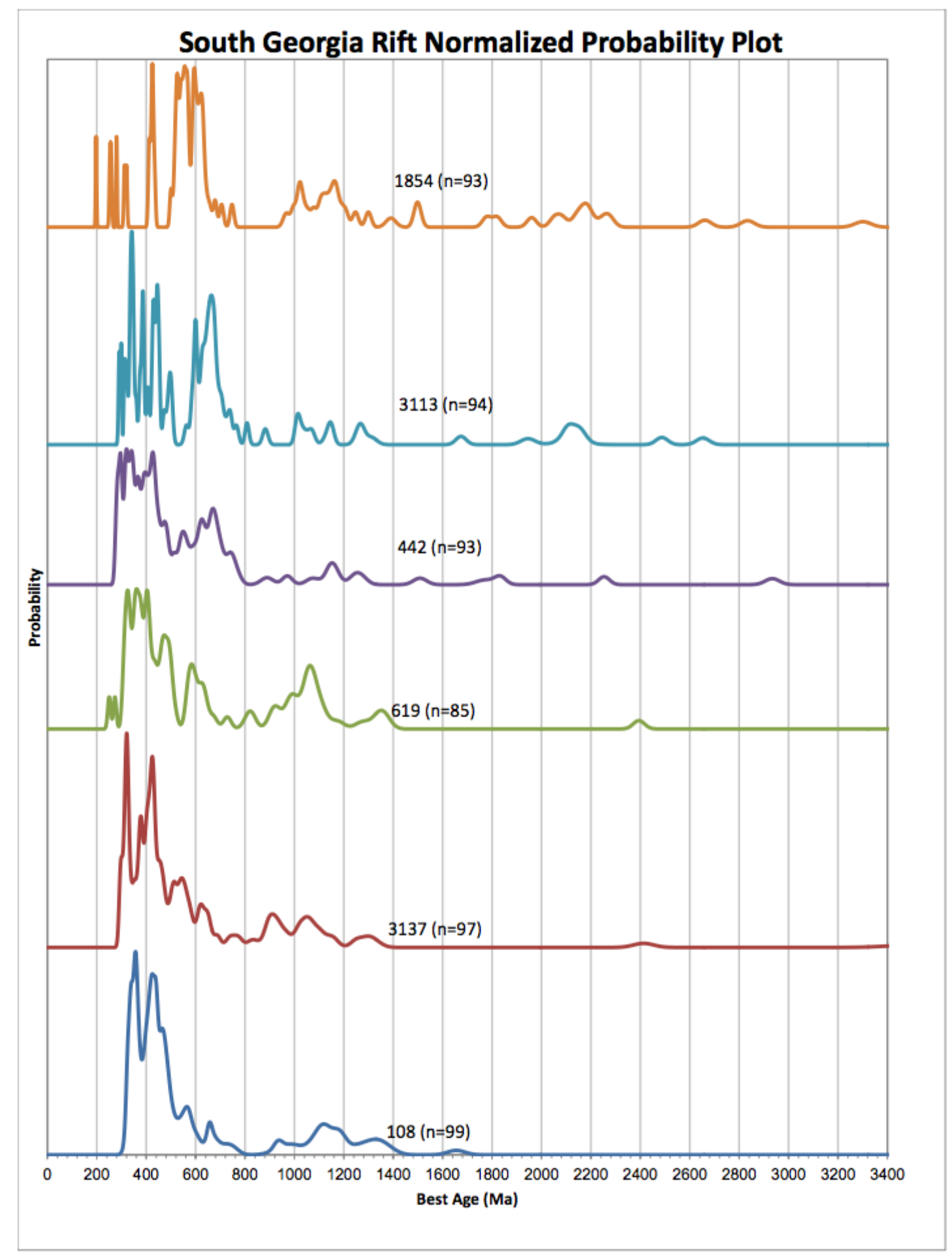

Figure 42. Normalized Probability Plot for the SGR. 


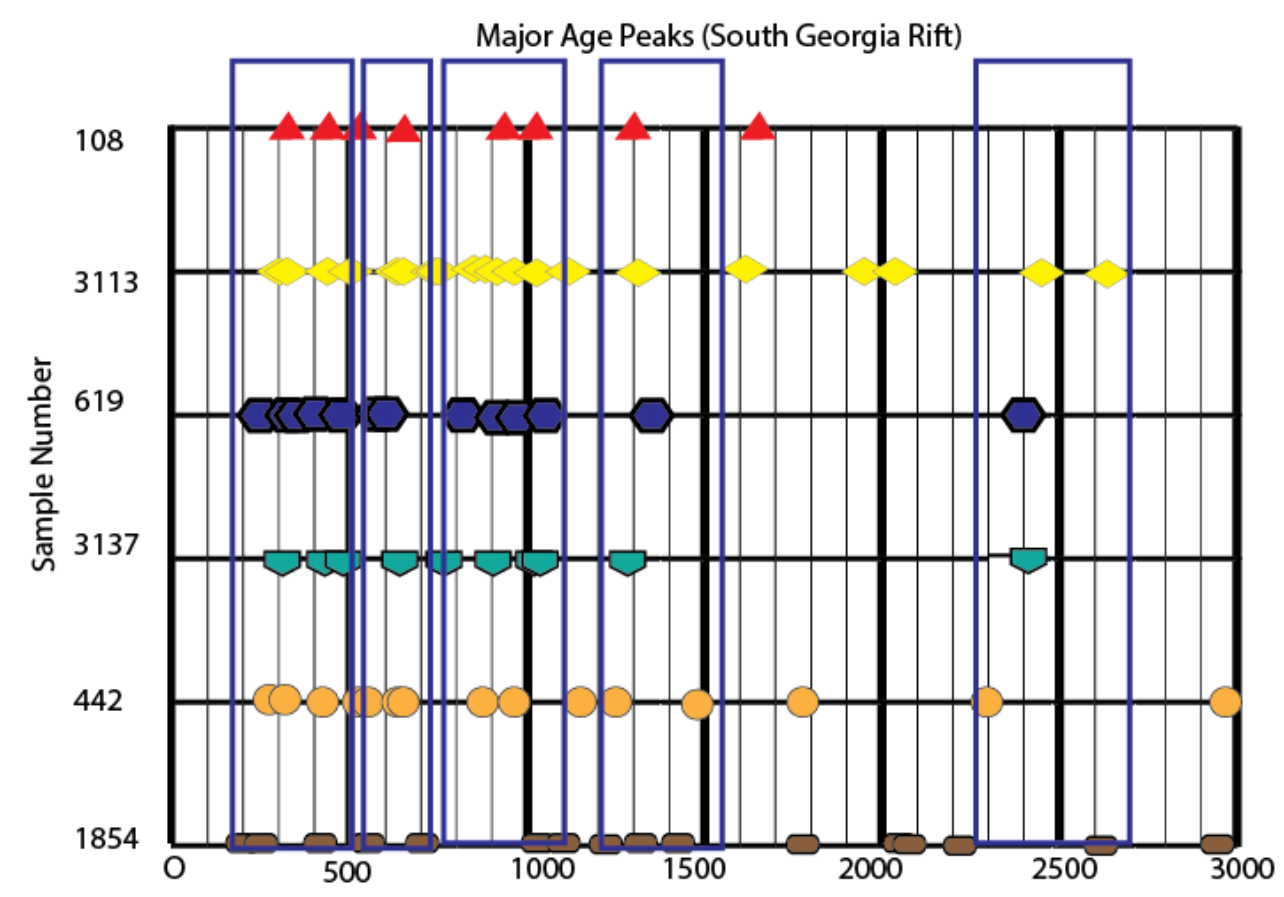

Age (Ma)

Figure 43. Major Age peaks for the SGR. Major age groups of peaks include 200-500 Ma, 525-725 Ma, 750-1100 Ma, 1200-1500 $\mathrm{Ma}$, and 2275-2600 Ma.

\section{Age Groupings (South Georgia)}

\begin{tabular}{|c|c|}
\hline Major Age Groupings (South Georgia) & Sample \\
\hline $200-500 \mathrm{Ma}$ & $\begin{array}{l}108 \text { (66 zircons, } 62.9 \%), 3113(39 \text { zircons, } 41.5 \%), 619(47 \\
\text { grains, } 54.7 \%), 3137 \text { (54 zircons, } 57.4 \%), 442(51 \text { zircons, } \\
54.8 \%), 1854(12 \text { zircons, } 13.3 \%)\end{array}$ \\
\hline $525-725 \mathrm{Ma}$ & $\begin{array}{l}108(14 \text { zircons, } 13.3 \%), 3113(31 \text { zircons, } 33.0 \%), 619(11 \\
\text { grains, } 12.8 \%), 3137(14 \text { zircons, } 14.9 \%), 442(24 \text { zircons, } \\
25.8 \%), 1854 \text { ( } 37 \text { zircons, } 41.4 \%)\end{array}$ \\
\hline 750-1100 Ma & $\begin{array}{l}108(7 \text { zircons, } 6.7 \%), 3113 \text { (7 zircons, } 7.4 \%), 619(18 \\
\text { zircons, } 20.9 \%), 3137(13 \text { zircons, } 13.8 \%), 442(5 \text { zircons, } \\
5.4 \%), 1854(7 \text { zircons, } 7.8 \%)\end{array}$ \\
\hline $1200-1550 \mathrm{Ma}$ & $\begin{array}{l}108(6 \text { zircons, } 5.7 \%), 3113(3 \text { zircons, } 3.2 \%), 619(4 \\
\text { zircons, } 4.7 \%), 3137(3 \text { zircons, } 3.2 \%), 442(3 \text { zircons, } \\
3.2 \%), 1854(6 \text { zircons, } 6.7 \%)\end{array}$ \\
\hline 2275-2600 Ma & $\begin{array}{l}108(0 \text { zircons, } 0 \%), 3113(1 \text { zircon, } 1.1 \%), 619(1 \text { zircon, } \\
1.2 \%), 3137(1 \text { zircon, } 1.06 \%), 442(1 \text { zircon, } 1.1 \%), 1854 \\
(1 \text { zircon, } 1.1 \%)\end{array}$ \\
\hline
\end{tabular}

Table 7. Major age groupings for the South Georgia Rift. 
Metamorphic zircon age populations were identified for the SGR samples based on a U/Th ratio greater than 5 (Fig. 44). Two age populations of metamorphic zircons were identified and are evident in all samples (Fig. 45; Table 8). Metamorphic zircon groupings range from 200-600 $\mathrm{Ma}$, and 900-1450 Ma. The percent of metamorphic zircons per sample ranges from $10.6 \%$ in sample 3113 to $35 \%$ in sample 108.

\section{Maximum Depositional Age}

The maximum depositional age for each sample is listed in Table 9. Maximum depositional age is based on the youngest grain from each sample (Dickinson and Gehrels, 2009). Typically, the maximum depositional age is based on the age of the youngest peak. Mesozoic zircon are very rare in these samples, so the youngest grain present was used to to determine max age instead. Maximum depositional ages for South Florida Basin range from 195$426 \mathrm{Ma}$ and from 197-235 Ma for the South Georgia basin. Maximum depositional ages in south Florida are consistent with ages reported for igneous intrusions in Florida, indicating that the Wood River Formation was likely deposited no later than 195-235 Ma. Maximum depositional ages for the South Georgia Rift are not considered indicative of depositional age, as only one grain was younger then $190 \mathrm{Ma}$, and the rest were greater than $300 \mathrm{Ma}$. This is considerably

older than the expected age of syn-rift deposition. Mesozoic magmatic activity likely did not contribute to the deposition in the South Georgia Rift during deposition of the Newark Supergroup, suggesting magmatic activity postdated deposition. 

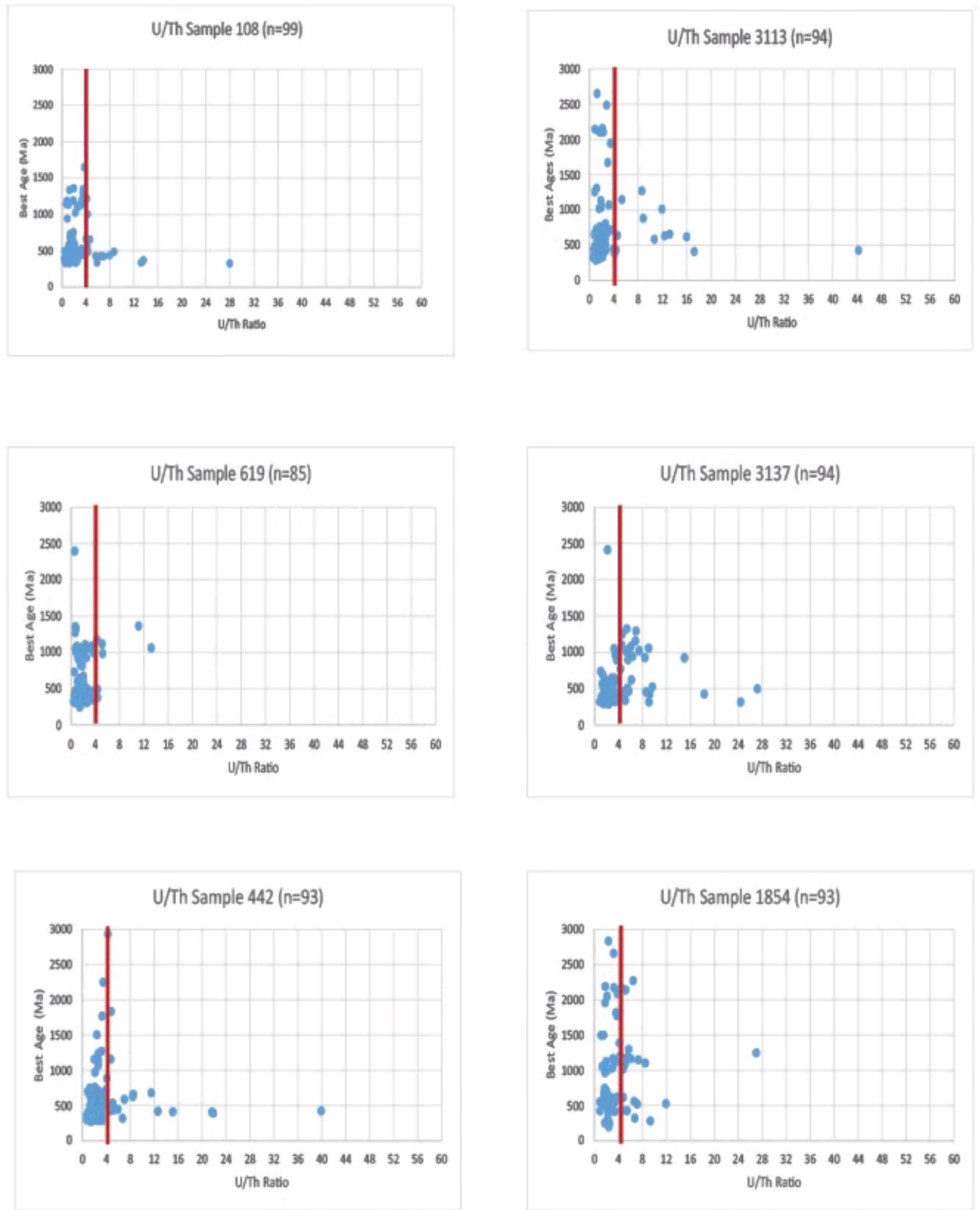

Table 44. Metamorphic zircons for the SGR. Metamorphic zircons were identified based on a U/Th ratio greater than 5 . 


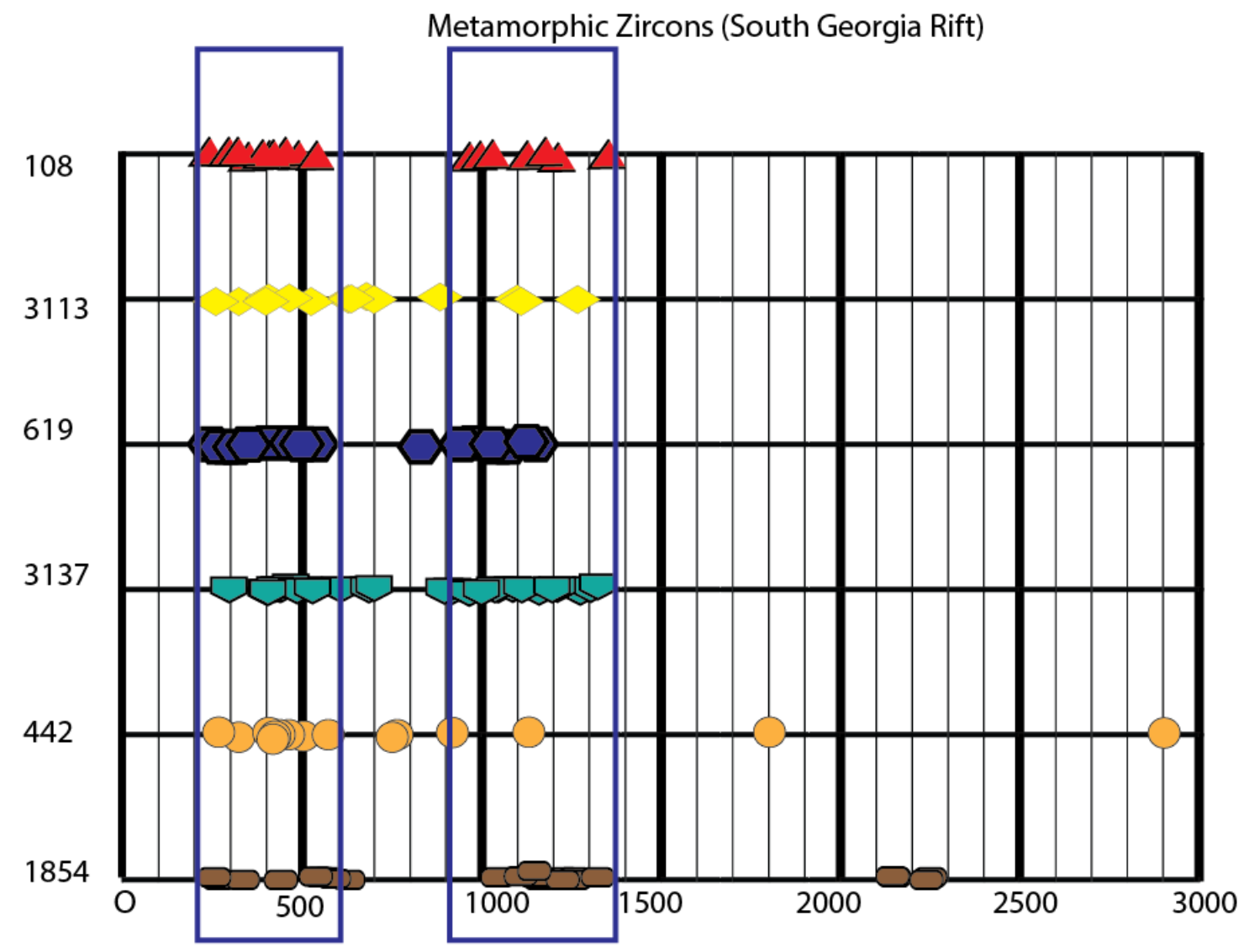

Figure 45. Major metamorphic peaks for the SGR. Major groupings are between 200-600 Ma, and 900-1400 Ma.

\begin{tabular}{|l|l|}
\hline $\begin{array}{l}\text { Age Group Metamorphic Zircons (South } \\
\text { Georgia) }\end{array}$ & Sample \\
\hline $200-600 \mathrm{Ma}$ & $108(30$ zircons, 28.6\%), 3113 (7 zircons, \\
& $7.5 \%), 619(12$ zircons, 14.0\%), 3137 $(15$ \\
& zircons, 16.0\%), 442 $(10$ zircons, $10.8 \%)$, \\
& $1854(7$ zircons, $7.8 \%)$ \\
\hline $900-1400 \mathrm{Ma}$ & $108(7$ zircons, 6.7\%), 3113 (3 zircons, 3.1\%), \\
& $619(4$ zircons, 4.7\%), 3137 (10 zircons, \\
& $10.7 \%), 442(2$ zircon $(1$ zircon, $1.1 \%), 1854$ \\
& $(8.9 \%)$ \\
\hline
\end{tabular}

Table 8. Major metamorphic zircon age groupings identified in the SFB. Major age groups present are 200-600 Ma, and 900$1400 \mathrm{Ma}$. 


\section{Age Group Metamorphic Zircons (South Georgia)}

\begin{tabular}{|l|l|l|l|l|l|l|l|l|l|l|l|l|l|}
\hline SFB & & & & & & & & SGR & & & & & \\
\hline$\#$ & ODP & $\mathbf{2 0 1 2}$ & $\mathbf{7 7 8}$ & $\mathbf{1 0 5 6 6}$ & $\mathbf{3 5 7 8}$ & $\mathbf{1 5 0 9 5}$ & & $\mathbf{1 8 5 4}$ & $\mathbf{4 4 2}$ & $\mathbf{3 1 3 7}$ & $\mathbf{6 1 9}$ & $\mathbf{3 1 1 3}$ & $\mathbf{1 0 8}$ \\
\hline $\begin{array}{l}\text { Youngest } \\
\text { grain } \\
\text { (Ma) }\end{array}$ & 473 & 234 & 201 & 112,240 & 426 & 195 & & 197 & 279 & 294 & 250 & 291 & 323 \\
\hline
\end{tabular}

Table 9: Maximum Depositional Age for basal clastics in the SFB and SGR.

\section{Correlation between Basins}

Comparison of normalized probability density plots for all samples included in this study reveal major age groupings include 300 to $500 \mathrm{Ma}$ in all of the SGR samples, as well as small peaks in 2012, 778, and 10566 of South Forlida (Fig. 46). Another common cluster is 500 to $700 \mathrm{Ma}$, which is present in all of the SFB samples, and peaks in samples 3113, 619, and 1854 from the SGR. SGR samples contain diverse age distributions, while samples from SFB typically show only one cluster of ages from 500 to 700 Ma. Cumulative density plots (CDP) were constructed to compare major age probabilities between samples. The greater the similarity between the curves, the more likely it is that those samples share a source.

A CDP plot for the SFB is displayed in Figure 47. For the majority of samples, $90 \%$ of the grains are younger than $\sim 700 \mathrm{Ma}$, aside from sample 2012. A CDP for the SGR is displayed in Figure 48. Major probability differences are noticeable around 500, 900, and 2000 Ma. Sample 1854 has significantly less grains around 500 and 900 Ma than the rest of the samples. 


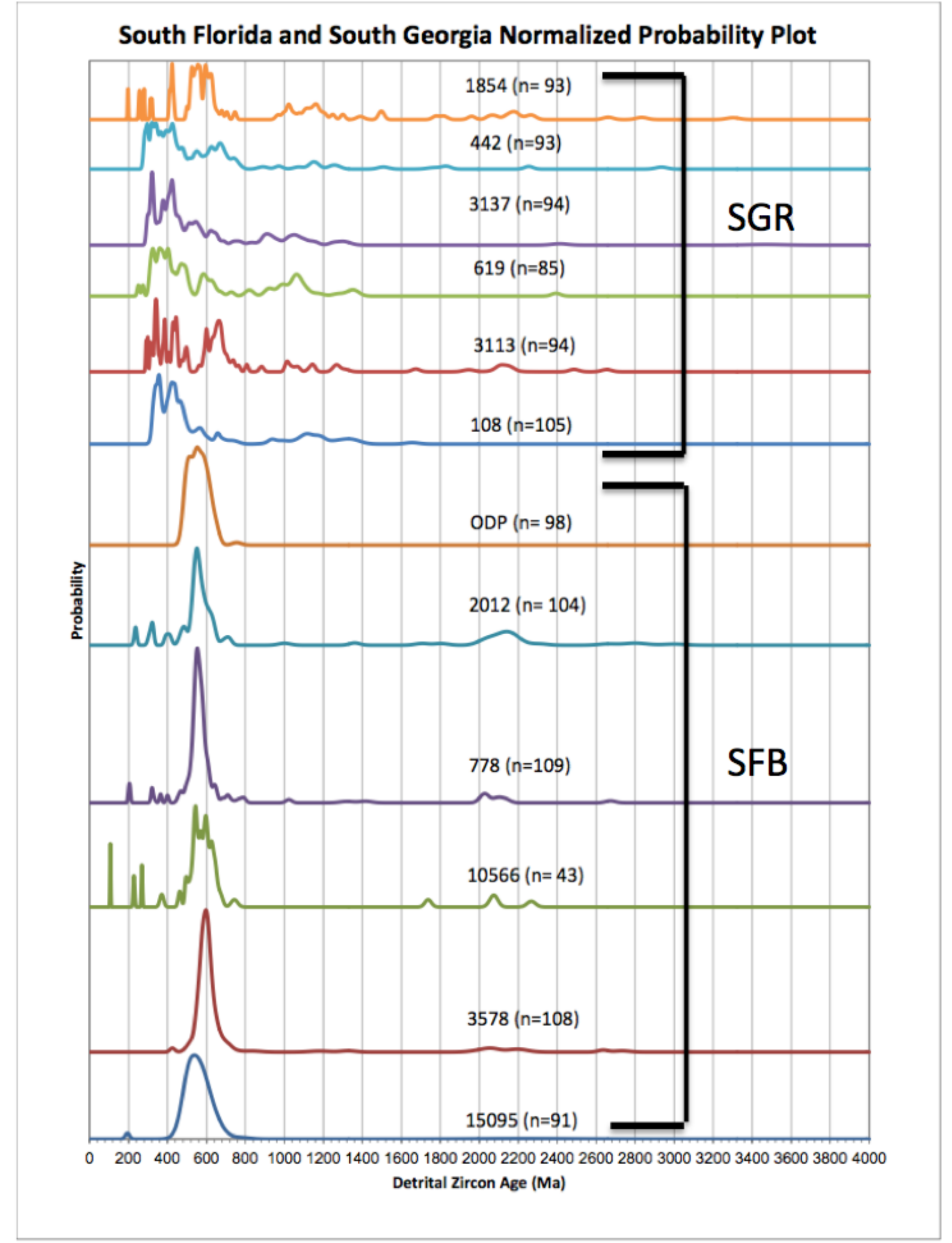

Figure 46. Normalized Probability Plot for the SGR and SFB. 
A CDP plot for the SFB is displayed in Figure 47. For the majority of samples, $90 \%$ of the grains are younger than $\sim 700 \mathrm{Ma}$, aside from sample 2012. A CDP for the SGR is displayed in Figure 48. Major probability differences are noticeable around 500, 900, and $2000 \mathrm{Ma}$.

Sample 1854 has significantly less grains around 500 and 900 Ma than the rest of the samples.

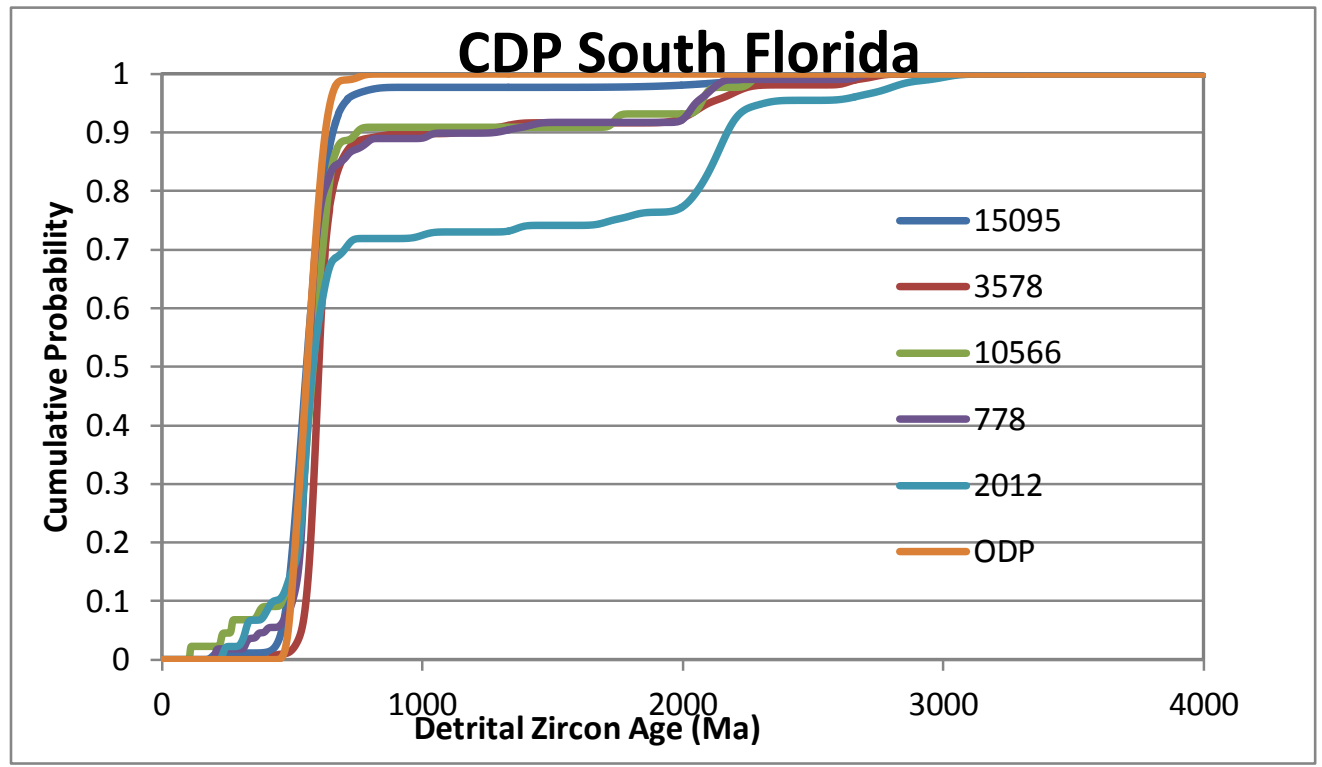

Figure 47. Cumulative density plot for SFB samples. 90\% of the samples are younger than 700 Ma, aside from sample 2012.

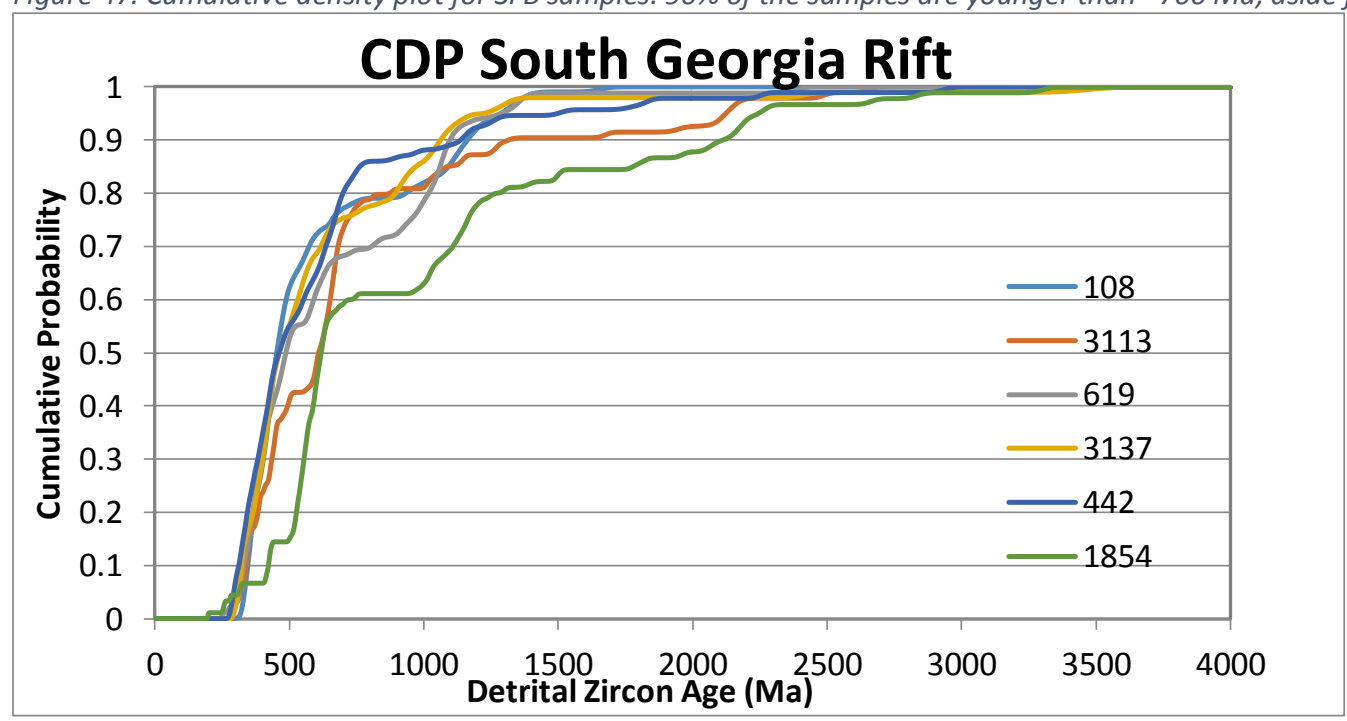

Figure 48. Cumulative density plot for the SGR. The largest differences are the abundances of the 500-600 Ma, 900 Ma, and 1500-2200 Ma age populations. 
The Kolmogorov-Smirnoff test (i.e., K-S test) is used to identify if two populations share a similar source based on population distribution. The test is based on the CDP plots constructed in Figure 46, 47, and 48. The K-S test calculates the probability difference between two samples $\left(\mathrm{D}_{\text {obs }}\right)$ and generates a probability $(\mathrm{P})$ value that reflects the probability that the samples came from the same source. If $\mathrm{P}$ is low, then the two samples being compared likely did not come from the same source. For the purpose of this study, a cut off $\mathrm{P}$ value of 0.05 is used. If the $\mathrm{P}$ value is higher than .05 , then there is $95 \%$ confidence that the samples are statistically similar. The $\mathrm{D}_{\mathrm{obs}}$ and $\mathrm{P}$ value can be highly dependent on the number of grains sampled, especially for samples with a diversity of ages; number of ages acquired for each sample should be roughly the same in order for the K-S test to be accurate.

The K-S test results for the SGR samples and SFB samples is displayed in Table 10. Within the SGR, samples are fairly statistically similar, aside from samples 3113 and 1854. Sample 3113 is statistically likely to be derived from a different source than the most other SGR samples, but shares similarities with samples 619 and likely 442. Sample 1854 is statistically different from all samples in the SGR, implying it does not share a similar source. Sample 619 and 442 are statistically similar to all of the samples, implying they share a source. Samples 108 and 3137 also are similar to all samples, aside from 3113 and 3137 respecively.

Samples from the SFB are not as statistically similar to each other, aside from sample 10566. Sample 10566 is statistically similar to all samples except 3578 . Sample 778 is similar to all samples, except 3578. Sample 15095 is statistically similar to all populations except for 3578 and 2012. With the exception of 2012 and 3578, zircon ages from sample ODP is similar to all other SFB samples, suggesting these rocks or similar rocks supplied sediment to the SFB. Sample 2012 is different from all samples, aside from 778 and 10566. Sample 3578 is 
statistically different from other samples in the basin. Based on the K-S test, samples from SFB and SGR are not statistically similar, aside from sample 2012 and 1854.

\section{$\underline{\text { K-S Test South Florida Basin and South Georgia Rift }}$}

\begin{tabular}{|c|c|c|c|c|c|c|c|c|c|c|c|c|}
\hline & \multicolumn{12}{|c|}{ K-S P-values using error in the CDF } \\
\hline & 108 & 3113 & 619 & 3137 & 442 & 1854 & 10566 & 3578 & ODP 77 & 778 & 2012 & 15095 \\
\hline 108 & & 0.002 & 0.443 & 0.805 & 0.543 & 0.000 & 0.000 & 0.000 & 0.000 & 0.000 & 0.000 & 0.000 \\
\hline 3113 & 0.002 & & 0.290 & 0.013 & 0.074 & 0.003 & 0.023 & 0.000 & 0.000 & 0.000 & 0.003 & 0.000 \\
\hline 619 & 0.443 & 0.290 & & 0.846 & 0.192 & 0.000 & 0.000 & 0.000 & 0.000 & 0.000 & 0.000 & 0.000 \\
\hline 3137 & 0.805 & 0.013 & 0.846 & & 0.882 & 0.000 & 0.000 & 0.000 & 0.000 & 0.000 & 0.000 & 0.000 \\
\hline 442 & 0.543 & 0.074 & 0.192 & 0.882 & & 0.000 & 0.000 & 0.000 & 0.000 & 0.000 & 0.000 & 0.000 \\
\hline 1854 & 0.000 & 0.003 & 0.000 & 0.000 & 0.000 & & 0.011 & 0.001 & 0.000 & 0.000 & 0.393 & 0.000 \\
\hline 10566 & 0.000 & 0.023 & 0.000 & 0.000 & 0.000 & 0.011 & & 0.030 & 0.396 & 0.447 & 0.270 & 0.597 \\
\hline 3578 & 0.000 & 0.000 & 0.000 & 0.000 & 0.000 & 0.001 & 0.030 & & 0.000 & 0.000 & 0.001 & 0.000 \\
\hline ODP & & & & & & & & & & & & \\
\hline 77 & 0.000 & 0.000 & 0.000 & 0.000 & 0.000 & 0.000 & 0.396 & 0.000 & & 0.273 & 0.001 & 0.980 \\
\hline 778 & 0.000 & 0.000 & 0.000 & 0.000 & 0.000 & 0.000 & 0.447 & 0.000 & 0.273 & & 0.082 & 0.146 \\
\hline 2012 & 0.000 & 0.003 & 0.000 & 0.000 & 0.000 & 0.393 & 0.270 & 0.001 & 0.001 & 0.082 & & 0.007 \\
\hline 15095 & 0.000 & 0.000 & 0.000 & 0.000 & 0.000 & 0.000 & 0.597 & 0.000 & 0.980 & 0.146 & 0.007 & \\
\hline
\end{tabular}

Table 10. K-S test for the SGR and SFB. A P value above .05 is considered statistically similar. SGR samples (108, 3113, 619, 3137, 442 , and 1854 are overall statistically different than samples from the SFB (10566, 3578, ODP, 778, 2012, and 15095).

\section{Thin Sections: South Florida}

The table of thin section data from southern Florida is displayed in Table 4. Sample descriptions are provided below. Coarse-grained samples were point counted and described using the Folk (1980) classification scheme (Figure 49). 
$\underline{\text { ODP }}$

Although no zircons were obtained from the clastic interval of the IODP wells 0537 and 0538, two thin-sections were made. No thin-sections were made for the basement section due to the small sample size, and to increase the chances of getting a usable number of zircons for dating.

Sample 1 was collected from ODP Site 0537 from Core 13R, section 1, and interval 95110 (Figure 50). It is interpreted as arkosic conglomeratic sandstone with calcite cement. Grains consist primarily of quartz and perthite fragments. Quartz grains are large (up to $1 \mathrm{~mm}$ ), and well rounded. Inclusions are present in several quartz grains. Calcite cements are sparry with a second phase of blocky calcite cement.

Sample 2 was collected from ODP site 77 hole 0538A, 034R, 01W, interval 54.57-57 (Figure 50). It is interpreted as mudstone from Deep Sea Drilling report (Dallmeyer, 1984). It consists of fine-grained mudstone with plagioclase, biotite, chlorite clays, and opaque minerals. Quartz veins are common, and occur at three different angles. En echelon fractures may be present. Ribbons of quartz are interpreted as interstitial quartz, which formed during low grade metamorphism (Buffler et al., 1984).

\section{$\underline{\text { South Florida Basin Clastics }}$}

Overall, SFB samples are 50-100\% quartz, 5-50\% feldspar, and typically less than 5\% lithics, except for sample 1411. Samples plot in the quartz arenite, subarkose region on a ternary diagram. Samples consist primarily of angular-subrounded monocrystalline quartz fragments. Feldspar fragments are predominantly plagioclase, and twinning is common (Figure 52). Minor twinned feldspar and orthoclase/microcline is also present. Lithic fragments are extremely uncommon, although some igneous fragments are present. Perthite is common in sample 1411, 
and present in samples 10566 (Figure 53). Perthtite is composed of a plagioclase core, with a mixed plagiocoase/orthoclase rim. Grain-size is fairly unimodal .4 mm, and ranges from .2-.5 $\mathrm{mm}$. Samples are typically sub-angular to angular (Figure 52). Porosity is highest in sample 1411 (>20\%), but typically ranges from 5\%-10\%. Compacted quartz is present, as well as overgrowth quartz cement. There is some secondary feldspar due to feldspar dissolution. Sedimentary structures cannot be recognized from the cuttings alone.

Other samples from wells 1655, 778, and 3578 are composed mainly of mudstone, and were not point-counted. Cutting samples were also not point-counted. These samples were very fine-grained mudstone. Muscovite fragments are common in shale fragments in fine-grained samples (3578). Sample 1655 cuttings consist of mixed shale and limestone clasts. Carbonate clasts consist of calcite cement with ooid fragments.

\section{$\underline{\text { South Georgia Rift }}$}

The table of thin-sections from the South Georgia Rift is displayed in Table 2. All samples from the South Georgia Rift collected were too fine-grained for point counting, but observations were recorded on grain-size and composition. The majority of the samples are composed of muscovite and feldspar fragments. Sample 442 and 3137 is composed primarily of quartz, as well as secondary muscovite and feldspar (Figure 54). Beds are fairly well laminated, with alternating layers of mud and small quartz fragments. Quartz grains range from 0.05-0.1 $\mathrm{mm}$ (Figure 54), and are very angular. The grain-size of quartz increases from layer to layer and although the up direction is not known, the sequence likely represents a coarsening or fining upward sequence (442-1). Fossils include bivalves, and chambered gastropods present in sample 442. Calcite fragments are present in some samples, and calcite fracture-fill is also present in sample 619. Opaque minerals, micas, and feldspars are present in sample 3137 (Figure 54). 


\section{South Florida}

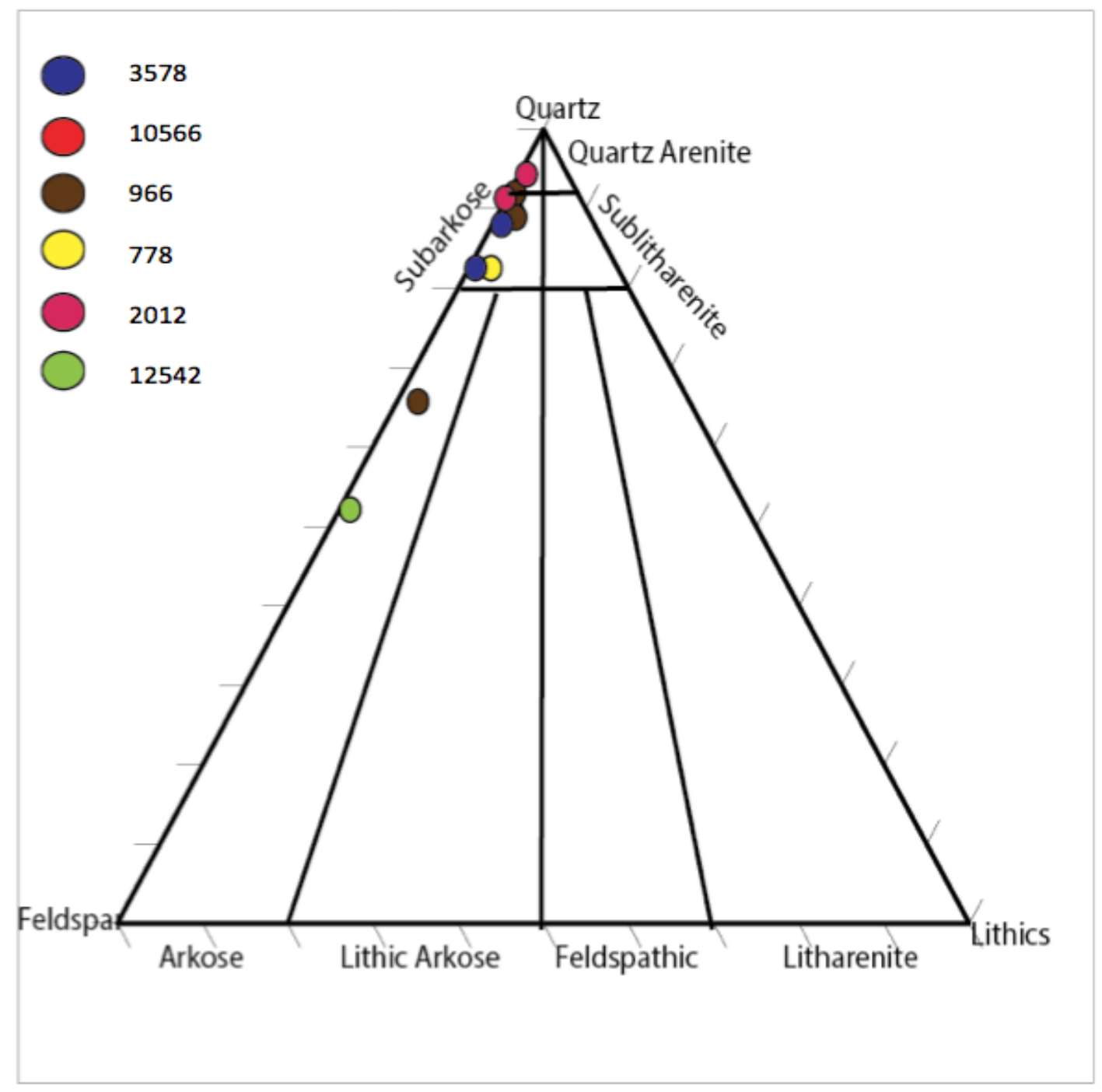

Figure 49. QFL plot of the Wood River and proximal clastics in the SFB. Samples fall predominantly in the subarkose range, although sample 12542 is lithic arkose. 


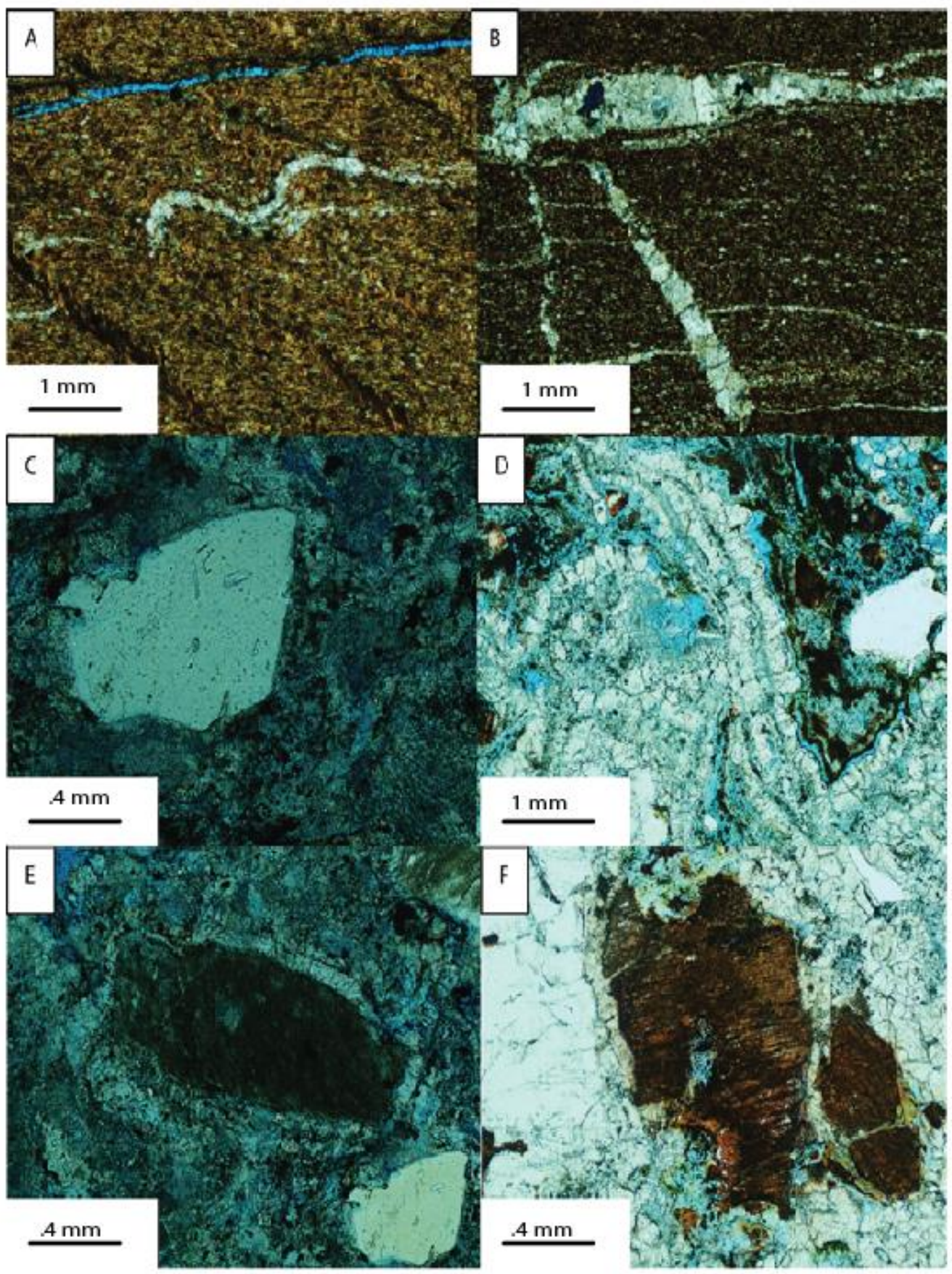

Figure 50. Thin sections. Thin sections from core. A: ODP-1. Folded quartz veins in mudstone. B: ODP-1. Calcite veins showing two directions of growth. C: ODP-2. Quartz grain with inclusions. D: ODP-2. Calcite veins showing two stage growth: First episode of micritic calcite, second stage blocky. E: ODP-2. Perthite fragment surrounded by blocky calcite cement F: ODP2. Perthite fragment surrounded by calcite cement. 


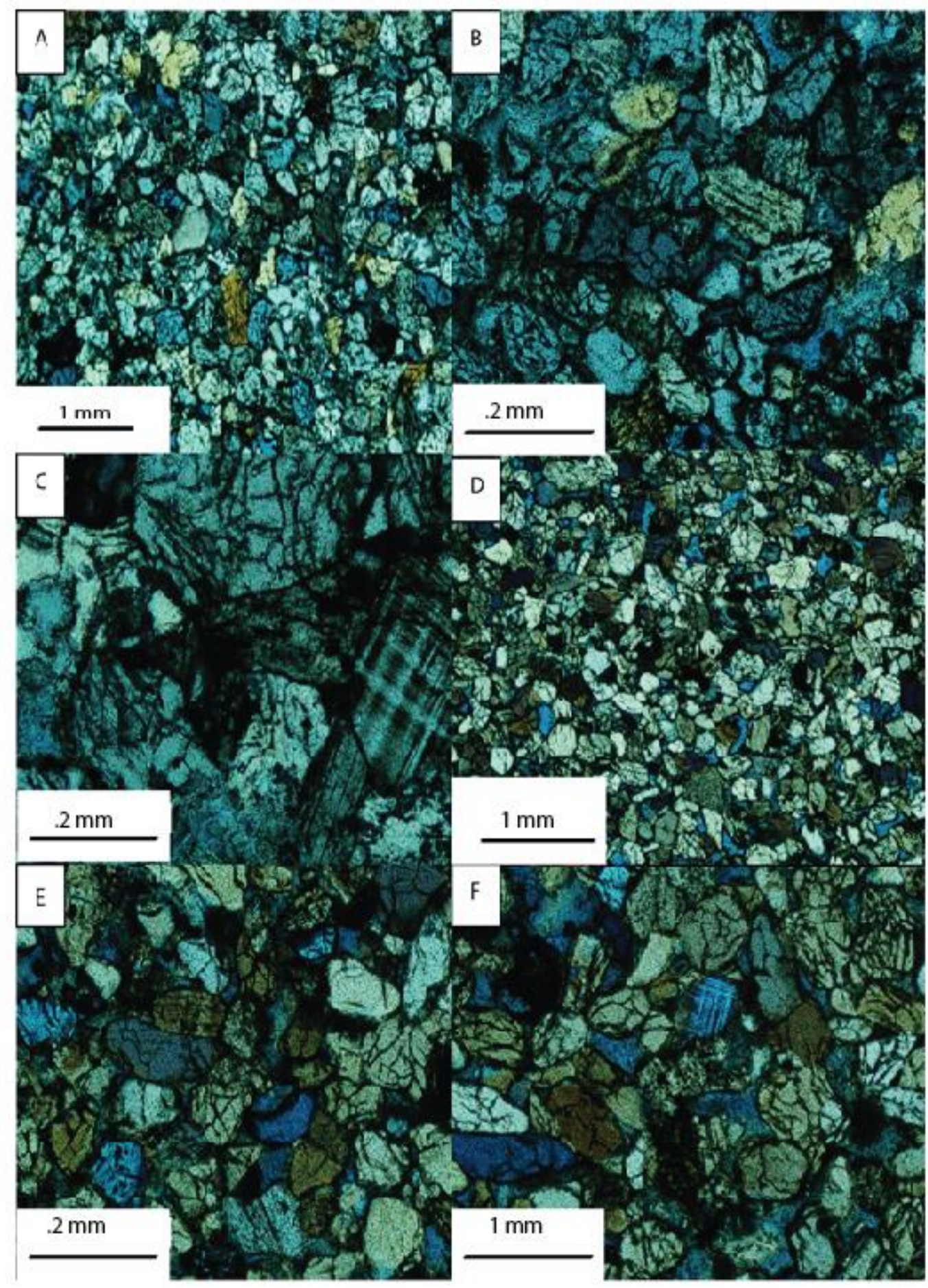

Figure 51. Thin sections. A: 10566 (from cuttings). Detrital quartz fragments. B. 10566. Quartz and feldspar (?) fragments. Well sorted, low porosity. C: 10566. Microcline surrounded by quartz. D: 778 (from cuttings). Detrital quartz fragments. E: 778. Detrital quartz and feldspar (?). F: 778. Detrital quartz and microcline. 


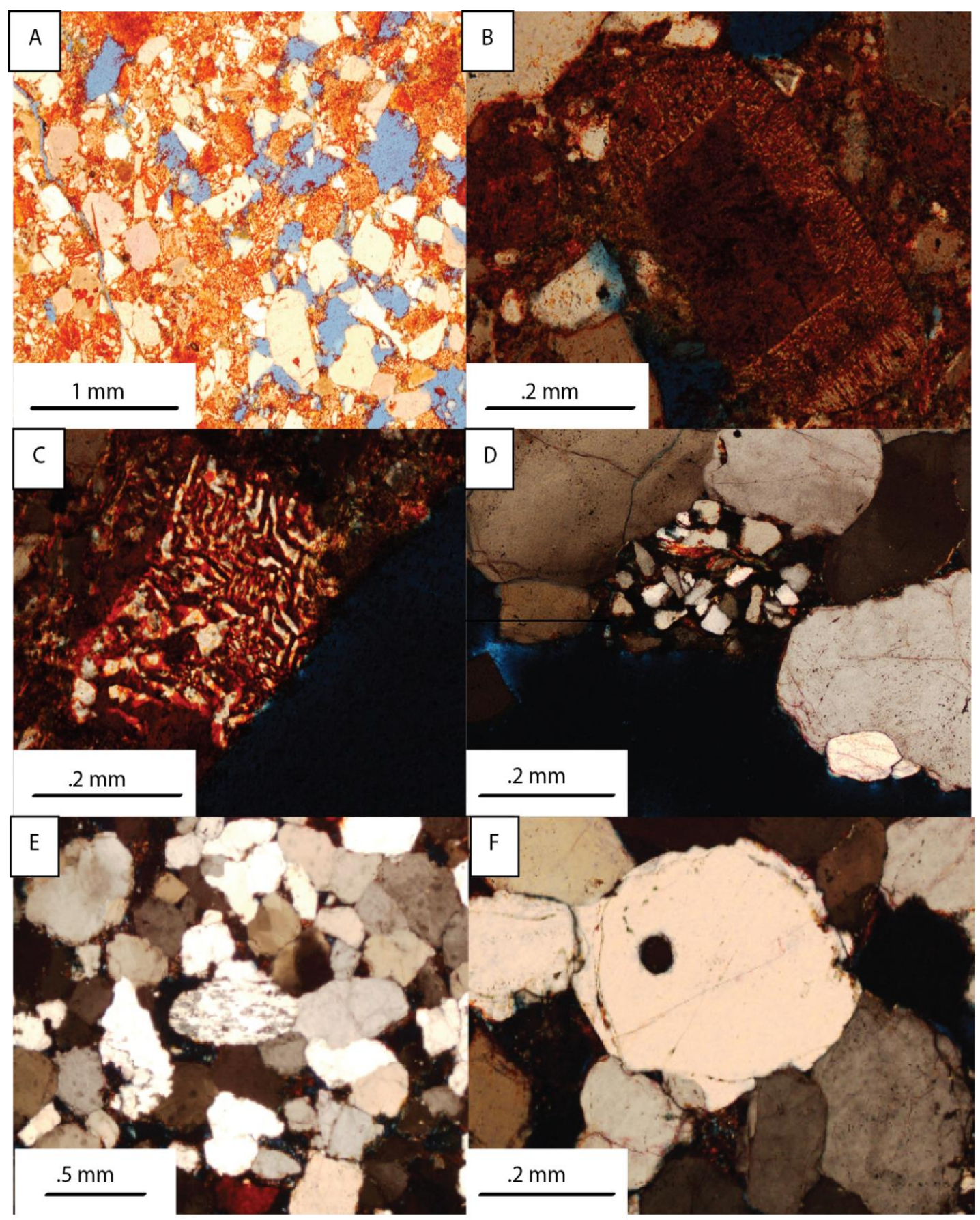

Figure 52. Thin sections. A: 12542 (from cuttings). Mix of feldspar (stained red) and detrital quartz fragments. B: 12542. Perthite with two stages of growth: plagioclase inner core, with mixed plagioclaselorthoclase outer rim. C: 12542: Perthite fragment with mixed plagioclase and orthoclase. D: 3578-1 (from sidewall core). Siltstone lithic fragment. E: 3578-1: Metamorphic fragment of quartzite (?) surrounded by quartz grains. F: 3578-1. Rounded quartz grain with vacuole and quartz overgrowth cement. 


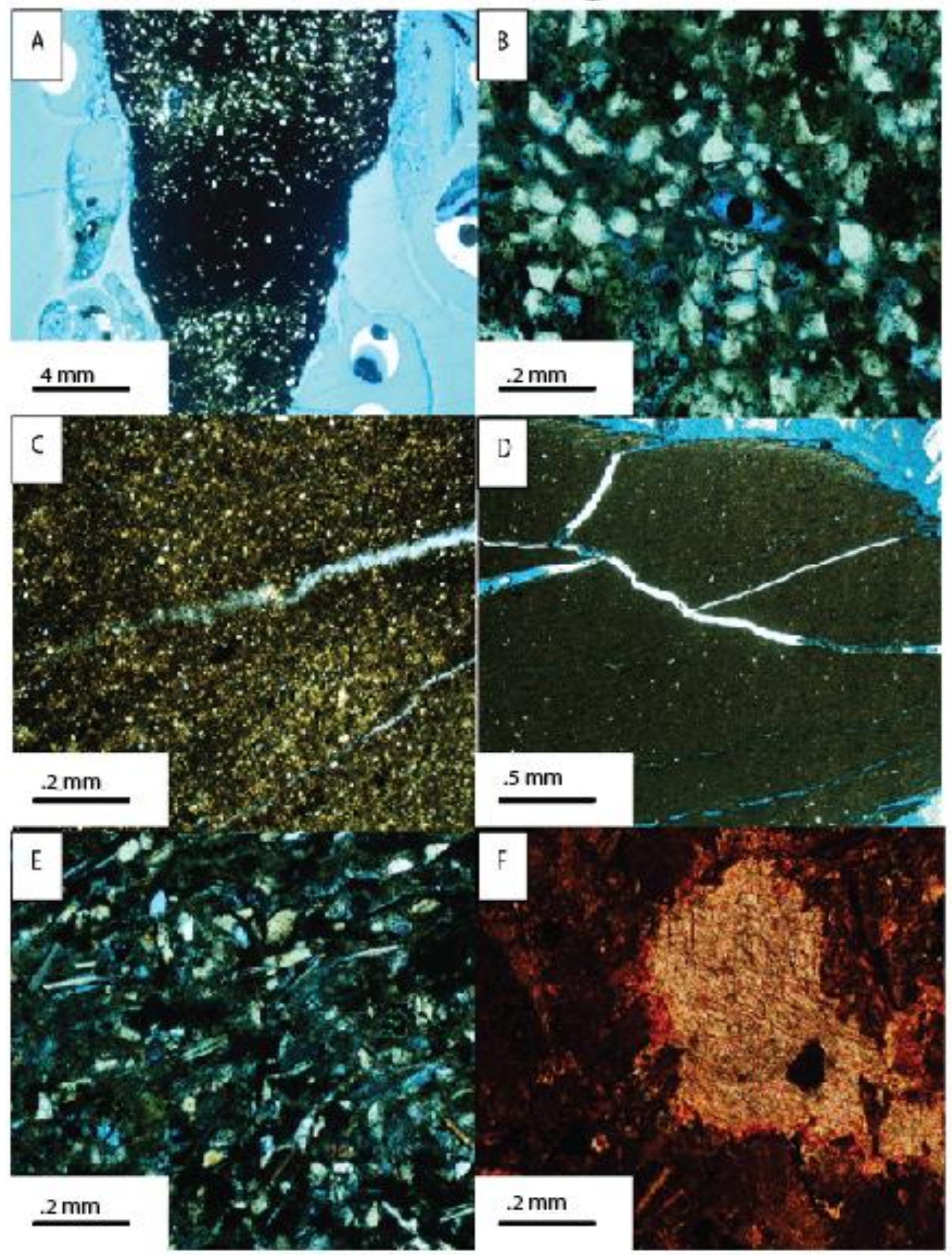

Figure 53. Thin sections. A: 442-1 (from cuttings). Shale with layers of fine-medium grain quartz that appear to coarsen up or down (up direction unknown). B: 442-2. Detrital quartz fragments with shell fragments. C: 108 (from cuttings). Quartz (?) fragment in fine grained shale. D: 619. Calcite (?) veins in fine grained shale. E: 3137 (from cuttings). Volcaniclastic quartz, feldspars, and micas, as well as opaques. F: 3137. Calcite cement. 


\section{Log Analysis}

0

\section{8}

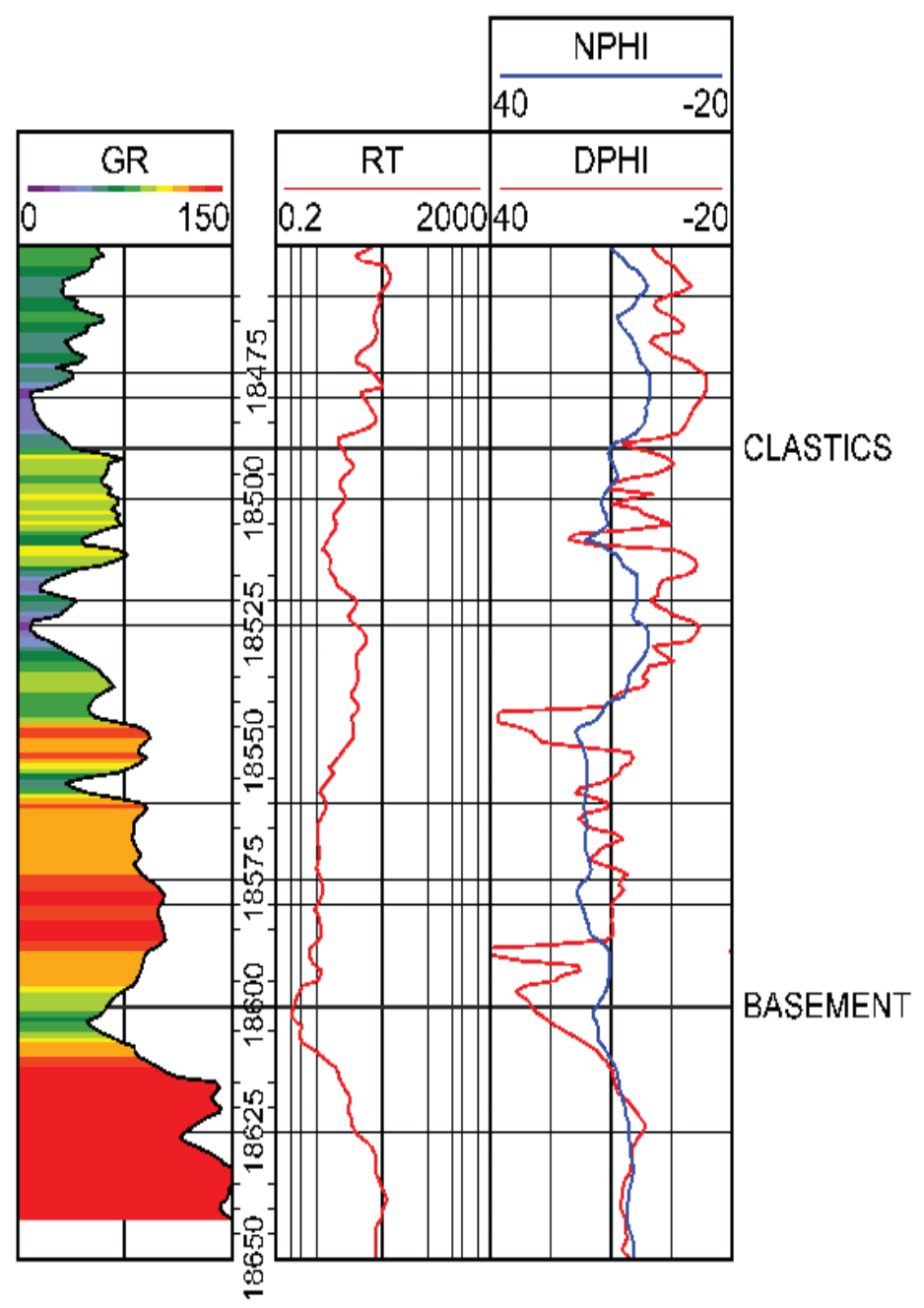

Figure 54. Electronic log from the type well 788. Clastic and basement tops are picked. Above the clastic surface is a transition to the limestone/dolomite interval of the Wood River.
Fifteen wells that tag clastics

and/or basement from the South Florida

Basin were evaluated to identify the depth

and thickness of the basal clastic deposits.

Logs were downloaded from the Florida

Occulus portal, and logs were digitized in

Petra. Few wells are present in South

Florida, and only a handful tag the basal

section and/or basement, making

correlation difficult. Log signatures for the

type well for the Wood River Formation is

displayed in Figure 54. Tops were picked

based on drillers log picks, mud logs, SP,

Gamma, Neutron/Density Porosity, and

Resistivity.

Lithologies were identified for the

basal shale, basal sandstone and overlying

carbonate/dolomite. The basal shale typically at the bottom of the Wood River Formation is is identified based on high SP and gamma ray signatures (100-150). Basal shale facies generally rest on basement of varying lithologies. The basal shale typically coarsens upward into a sandstone. 
The basal section of the Wood River Formation contains a basal sandstone unit.

Sandstone facies were identified based on low gamma and SP log signatures. Serrations in the SP and gamma ray curve indicate immature sandstone composition. Some curve signatures indicate large-scale fining and coarsening-upward sequences. Bell shaped curves are also present, as the facies shifts upward from shale, to sandstone, to dolomite.

The majority of the Wood River Formation consists of thick Dolomite with interbedded limestone layers, which is present in all wells. The base of the dolomite/limestone facies grades from a basal sandstone/shale to a dolomite, marked by a gradual increase in the SP and resistivity curves. Limestone and dolomite were difficult to identify from gamma ray logs but typically have a higher SP and resistivity values than sandstone with decreased porosity. Anhydrite layers are also common, which were identified based on a sharp spike in the SP and the resistivity.

Based on the picked tops, a depth to the clastic rock interval of the Wood Formation surface was constructed (Figure 56). A depth to basement map was not considered reliable, as very few wells penetrate into basement. The depth to the surface increases towards the south, indicating the SFB is likely deepest in the southern tip of Florida. An isochore map was constructed for the few wells that penetrate the entire section (Figure 57). Thickness appears to increase towards the northern part of the basin, but complete penetration through the Wood River is poor. $\mathrm{V}$-shale was calculated for the samples to identify changes in lithology (Figure 58). 


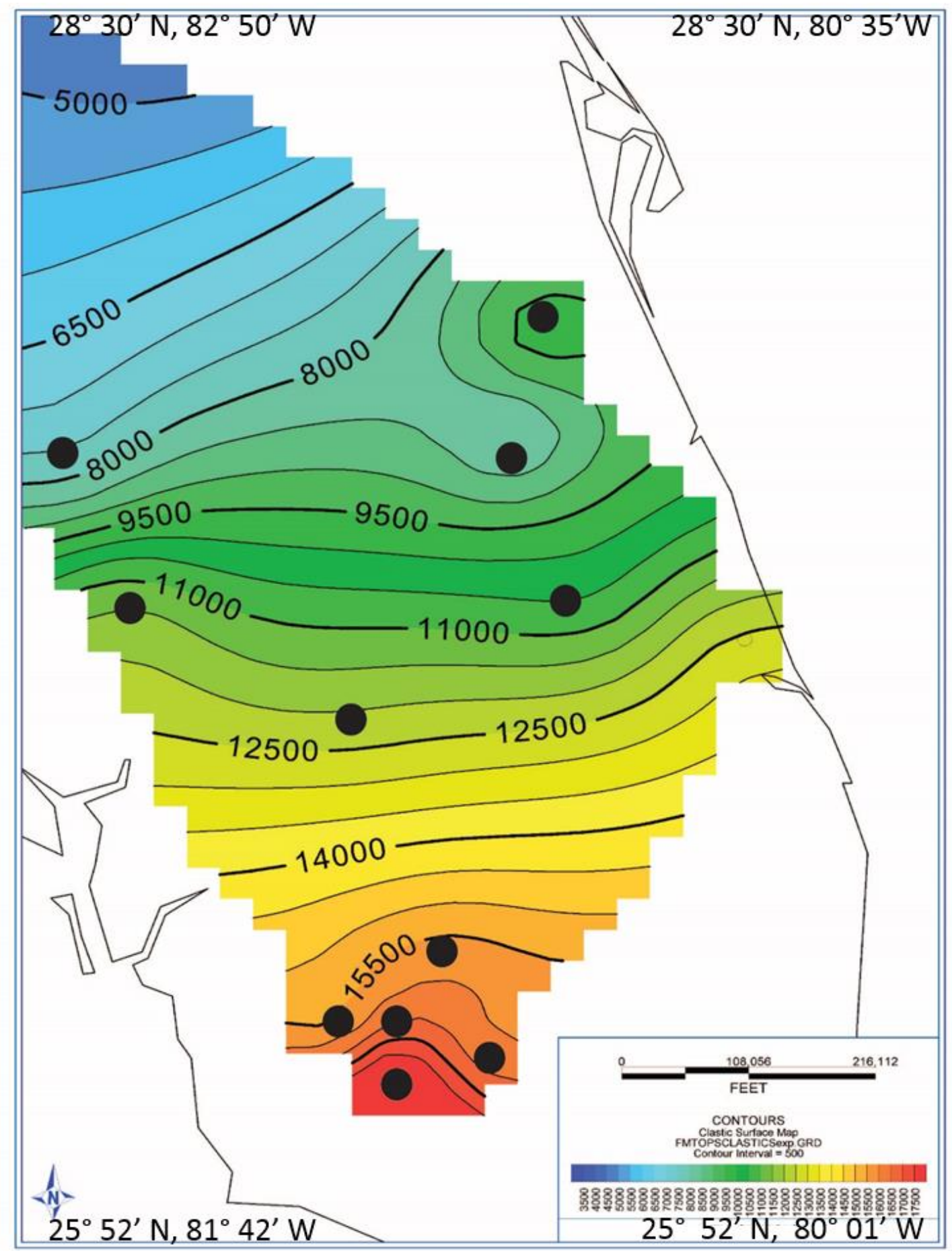

Figure 55. Map of the top of clastic surface in the SFB. The depth of the surface increases toward the south. 


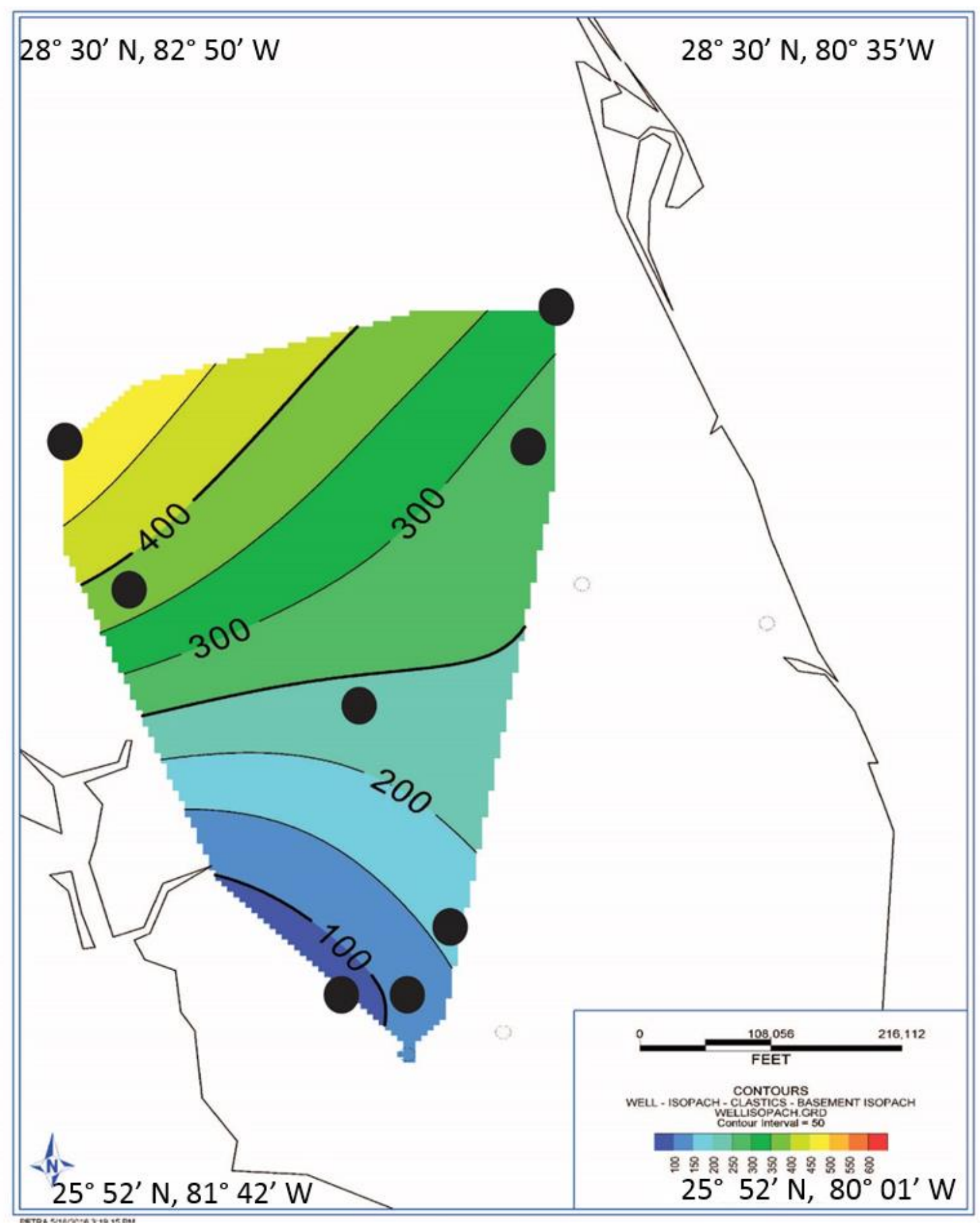

Figure 56. Isochore map calculated for the basal clastic succession. Clastic succession thickens toward the north. 


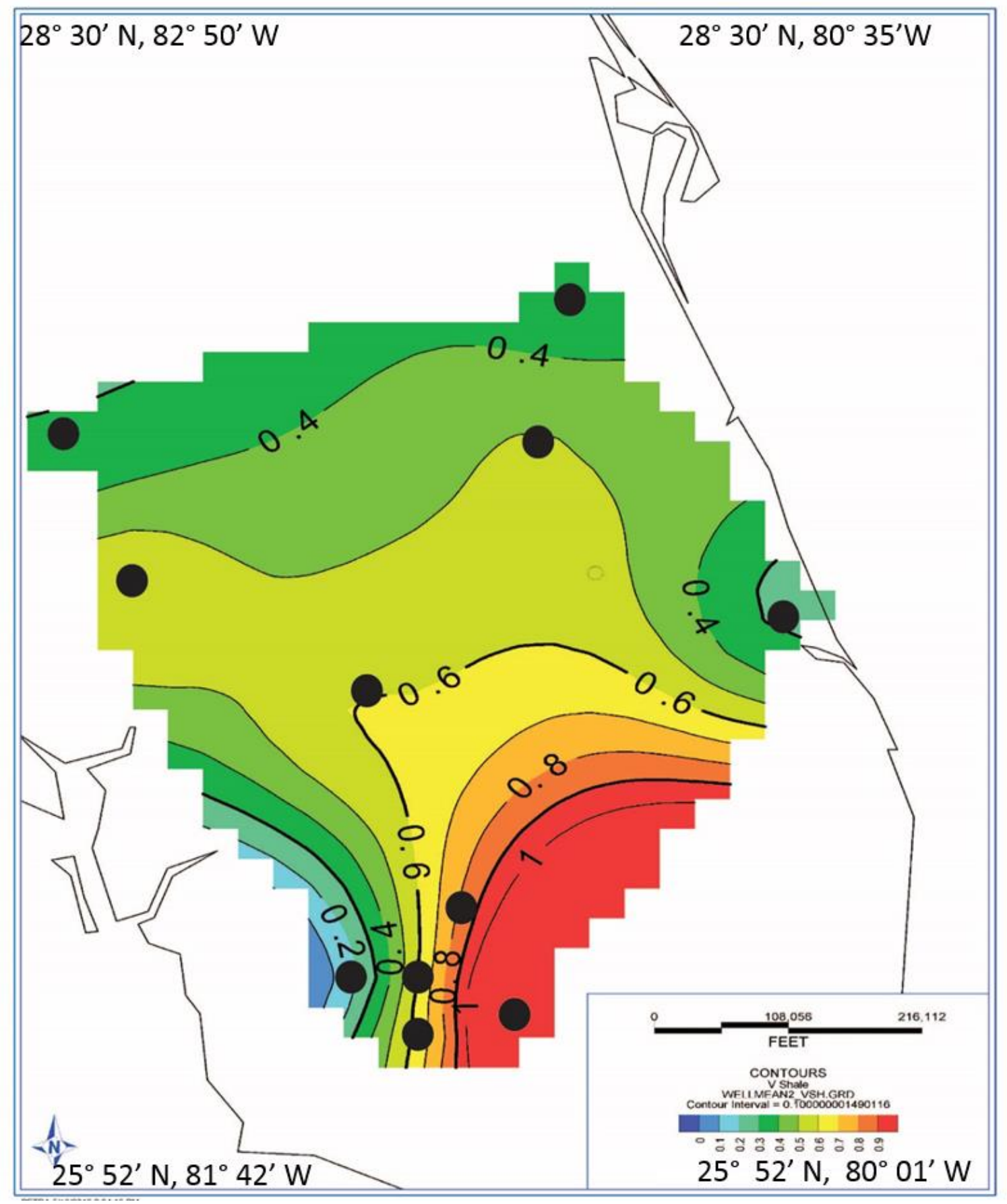

Figure 57. V-shale map calculated for the lower clastic succession in the SFB. Clastic v-shale generally increases to the south. 


\section{Chapter 5: Discussion}

\section{Potential Source Terranes}

Locations of potential source terranes of North America are displayed in Figure 58 and zircon forming tectonic events are listed in Table 11, and are discussed below.

\section{Tectonic Terranes of North America}

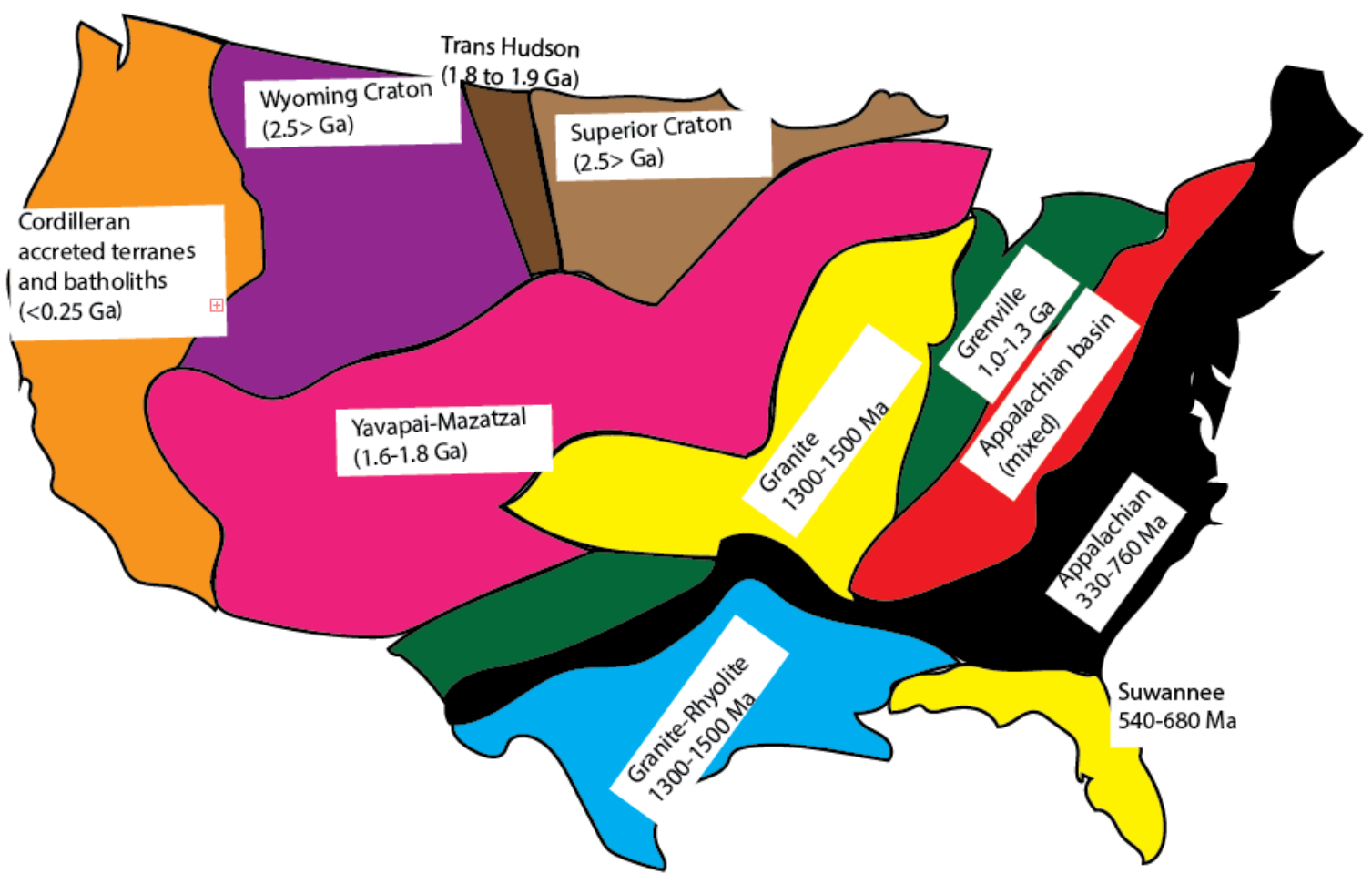

Figure 58. Terrane map of North America. Modified from Dickinson and Gehrels (2009). 
Potential Sources for the SFB and SGR

\begin{tabular}{|l|l|l|}
\hline Age (Ma) & Potential Sources & Sources \\
\hline $150-250$ & Pangea Rifting & $\begin{array}{l}\text { Mueller et al. (1994); Heatherington et } \\
\text { al. (1999); Applegate et al. (1981); } \\
\text { Hames et al. (2000) }\end{array}$ \\
\hline $265-330$ & Alleghenian & $\begin{array}{l}\text { Miller et al. (2006); Heatherington et } \\
\text { al. (2010); Schwartz et al. (2011) }\end{array}$ \\
\hline $350-420$ & Acadian Orogeny & Miller et al. (2000); Sinha et al. (2012) \\
\hline $430-490$ & Taconic Orogeny \& Cherokee orogeny & $\begin{array}{l}\text { Miller et al. (2000)., Moecher et al. } \\
\text { (2004)., Sinha et al. (2012) }\end{array}$ \\
\hline $525-680$ & $\begin{array}{l}\text { Gondwanan (Pan-African and } \\
\text { Brasiliano) }\end{array}$ & $\begin{array}{l}\text { Dallmeyer and Villeneuve, (1987); } \\
\text { Dallmeyer, (1989), Delor et al. (2002) }\end{array}$ \\
\hline $\begin{array}{l}\text { 430-460, 525-680, 700, 900-1300, } \\
1900-2200\end{array}$ & Peri-Gondwanan Terranes & $\begin{array}{l}\text { Bream et al. (2004); Carter et al., } \\
\text { (2006); Merschat and Hatcher, (2007); } \\
\text { Hatcher, (2010); Merschat et al. (2010); } \\
\text { Steltenpohl, (2010) }\end{array}$ \\
\hline $700-760$ & & $\begin{array}{l}\text { Goldberg et al. (1986); Fetter and } \\
\text { Goldberg, (1995); Aleinikoff et al. } \\
\text { (1995) }\end{array}$ \\
\hline $900-1300$ & Mount Roger & Rivers, (2008); Park et al. (2010) \\
\hline $1900-2200$ & & Mueller et al. (2014) \\
\hline
\end{tabular}

Table 11. Potential sources and associated zircon ages that may have sourced the SFB and SGR.

\section{Laurentia and associated sources}

Grenville orogen: The Grenville orogen is present throughout the eastern United States, west of the Appalachians and east of the Superior Province (Figure 58). It extends into South America south of the Amazonian Craton where it is referred to as the Sunsas Belt (Tohver et al., 2016). The Grenville orogen formed during the collision of the Amazonia continent with Laurentia and the assembly of Rodinia between 900-1300 Ma (Keppie and Ortega-Gutierrez, 1999). The Grenville is divided into three units by Rivers et al. (1989), including the Parautochthonous Belt, which consists of deformed Superior Province foreland basin strata and was metamorphosed around 980-1700 Ma, an Allochtonous Polycyclic Belt, with thrusting around (1050 M) and an older Allochthonous Monocyclic Belt with rocks younger than $1350 \mathrm{Ma}$ (Rivers et al., 1989; McLelland et al., 2010). 
A fragment of the eastern Laurentian margin composed of Grenville crustal rocks and overlying strata were involved in younger orogenic events and ultimately formed the Blue Ridge. The Blue Ridge was uplifted during the Alleghanian orogeny when Gondwana collided with Laurentia (Merschat et al., 2010). The Blue Ridge extends from northern Alabama/Georgia to central Maryland. The Blue

Map of Sample Locations and Triassic Basins of the Eastern United States

Ridge is composed

predominantly of granites,

limestones, and metamorphosed

volcanic and sedimentary

metasedimentary rocks, as well as

Paleozoic felsic and mafic

volcanic rocks (Hatcher et al.,

1989; Merschat et al., 2010).

Several terranes compose the

Blue Ridge including the

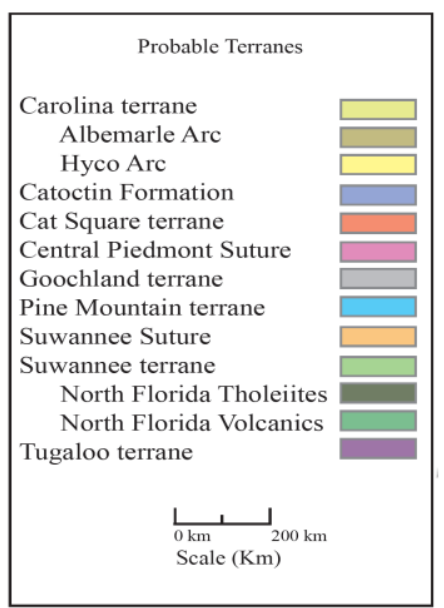

Figure 59. Potential Tectonic Peri-Gondwanan Terranes along the eastern margin of Laurentia. From Bowman and Weislogel. (2015).

Tugaloo, Cartoogechaya,

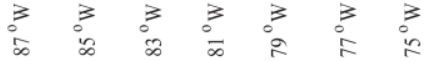

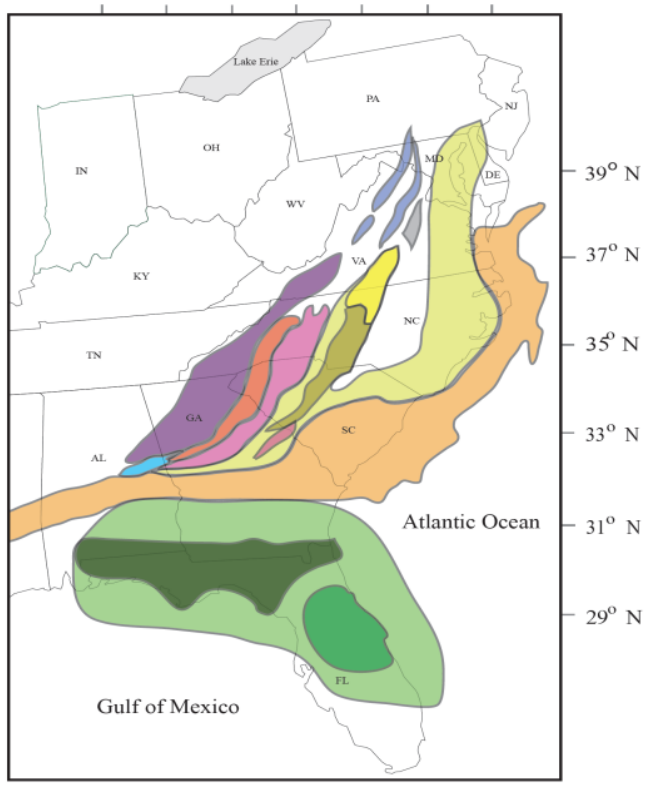

Cowrock and Cat Square (Fig. 59; Hatcher et al., 2007). These terranes consist primarily of metamorphosed sandstone, schist, gneiss and quartzite along with Neoproterozoic to Cambrian volcanic rocks that formed during the breakup of Rodinia (700-760 Ma; Hatcher et al., 2005; Burton and Southworth, 2010). Zircon ages in Blue Ridge units are dominated by $1100 \mathrm{Ma}$ zircons, as well as smaller abundances of $2900 \mathrm{Ma}, 2700 \mathrm{Ma}, 1900-2200 \mathrm{Ma}, 1300-1500 \mathrm{Ma}$, $1100 \mathrm{Ma}$, and 600-700 Ma zircon ages (Merschat et al., 2010; Hatcher, 2010). The southernmost 
known exposure of Grenville associated rocks is found in the Pine Mountain window. The Pine Mountain window is located in north-central Georgia, and is bound by the Charleston Terrane/Carolina Superterrane to the north and the Suwannee Terrane to the south (Fig. 59; Steltenpohl et al., 2010). The rocks exposed in the Pine Mountain window are interpreted to consist primarily of Grenville (1100 Ma) aged gneiss, and granite charnockites (Odom and Fullagar, 1973; Odom et al., 1984; Steltenpohl et al., 2010).

Rodinia Rifting: Evidence of Neoproterozoic rifting of Rodinia consists of a series of dike swarms present throughout the middle and eastern United States (Burton and Southworth, 2010). Dike swarm ages range from 700-760 Ma (Goldberg et al., 1986; Fetter and Goldberg, 1995; Aleinikoff et al., 1995; Ownby et al., 2004; Burton and Southworth, 2010). Zircon crystals formed from this rifting event are found as detrital grains reworked in early Paleozoic rocks contained in the eastern Blue Ridge.

Taconic Orogen: The Taconic orogen formed by subduction and volcanic arc development along the Laurentian margin between 430 and 490 Ma (Miller et al., 2000; Moecher et al., 2004, Sinha et al., 2012; Hatcher, 2010). The Taconic orogen extends in to the southern Appalachians, mainly represented by granitoid plutons of the Eastern Blue Ridge that contain 460 Ma zircon (Miller et al., 2006; Hatcher et al., 2007). A few Ordovician plutons (430 to 465 Ma) that intrude into Taconic structures have been identified throughout northern Georgia and South Carolina (Wilson et al., 2001; Miller et al., 2006).

Acadian Orogeny: Plutonism and structure associated with the Acadian orogeny is not prevalent in the southern Appalachians aside from a suite of plutonic rocks in the Carolina Terrane, which have been dated between 415-421 Ma (Samson and Secor, 2000; Hatcher et al., 2005; Hibbard et al; 2010). Along the eastern margin of US, Acadian subduction-related 
plutonism occurred between 350-420 Ma; these Acadian plutons are common in the central and northern Appalachian regions, but are rare in the southeastern US (Miller et al., 2000; Sinha et al., 2012). Granites associated with the Acadian Orogeny are present in the Salisbury plutonic suite of Georgia and North and South Carolina (Butler and Fullagar, 1978; McSween et al., 1991). A Famennian metamorphic event in the southern Appalachians also occurred between 375-345 Ma (Merschat et al; 2005).

Alleghenian: The Alleghany orogen formed from subduction and terrane accretion along the eastern margin of Laurentia from 265-330 Ma (Miller et al., 2006; Schwartz et al., 2011). The Alleghanian orogeny is associated with the closing of the Theic ocean, and oblique collision along the eastern margin of Laurentia, with southward suture propagation from 340-380 Ma (Fullagar and Butler, 1979; Speer et al., 1994; Coler et al., 1994; Wortman et al., 2000; Hatcher, 2010; Mueller et al., 2014). In the southeastern US, Alleghany orogenesis culminated with collision of the Suwannee Terrane (Hatcher, 2010; Mueller et al., 2014). Magmatism of the Alleghenian orogeny is rare in the southern US, and is expressed as granitoids both in eastern Laurentia and the northern Suwanee Terrane that range in age from 270-335 Ma (McSween et al., 1991; Speer et al., 1994; Heatherington et al., 2010). These ages are supported by previous $293 \mathrm{Ma}$ age dating determined from ${ }^{40} \mathrm{Ar} /{ }^{39} \mathrm{Ar}$ thermochronology of plutons in southern Alabama near the Suwannee suture (Thomas et al., 1989).

Alleghany Foreland Basin: Alleghanian clastic wedge deposits extend from central Pennsylvania to northern Georgia (Hibbard et al., 2010). Sediment shed into the Appalachian foreland basin were derived mainly from Laurentian sources dominated by Taconic, Acadian, and Alleghanian signatures, as well as other western sources (e.g., Mazatzal, Rhyolite, etc; Becker, 2005; Park, 2010) In particular, Acadian signatures are prevalent in the Alleghanian 
wedge, implying recycling of grains from older orogenic events, likely contained within older clastic wedge units involved in the Alleghany fold-thrust belt. However, small populations of 530-680 Ma detrital zircon were detected in the Acadian wedge, implying that some material originated from the Carolina Terrane or other Peri-Gondwanan Terranes and served as sources of sediment by the Devonian.

\section{Peri-Gondwanan Terranes}

A series of terranes derived from Gondwana were accreted to eastern Laurentia during the early to mid-Paleozoic (Fig. 59; Hatcher, 2010). The Peri-Gondwanan Terranes are dominated by igneous and volcanogenic sedimentary deposits with abundant 550 to $680 \mathrm{Ma}$ zircons (Hatcher, 2010). In addition to Neoproterozoic zircon, some Peri-Gondwanan terranes (e.g., Cat Square Terrane) contain older zircon derived from Laurentia (430 Ma, $460 \mathrm{Ma}$ ), indicating sediment transfer by Laurentian sedimentary systems to these Gondwanan crustal fragments (Merschat et al., 2010).

Cat Square Terrane: The Cat Square Terrane lies to the west of the Carolina Terrane and east of the Blue Ridge. The Cat Square Terrane consists of deep-water sandstones that were accreted along the east of the Tugaloo Terrane due to the westward subduction of the Carolina Terrane, and closing of the Cat Square ocean during the late Paleozoic (Merschat and Hatcher, 2007; Merschat et al., 2010). The Cat Square and Tugaloo Terrane have been metamorphosed into schists and gneiss, with zircon rims within the Cat Square indicate peak metamorphism between 350-360 Ma, coincidental with collision of the Carolina Terrane (Mershat et al., 2010). The Cat Square and Tugaloo received input from the Carolina Terrane and Laurentia, as indicated by zircons ages dominated by 1,100-1,400 Ma ages, but also include $430 \mathrm{Ma}, 500 \mathrm{Ma}$, 
$600 \mathrm{Ma}, 1,900-2,200 \mathrm{Ma}$, and 2,700-2,900 Ma (Bream et al., 2004; Carter et al., 2006; Mershat et al., 2010).

Carolina Superterrane: The Carolina Superterrane consists of a series of Peri-Gondwanan Terranes that accreted onto eastern Laurentia during the Paleozoic (Hatcher, 2010; Mershat et al., 2010; Figure 64) including the Charlotte Terrane, Uchee Terrane, Charleston Terrane and Milledgeville Terrane. Overall, The Carolina Superterrane is dominated by volcanic plutons and mafic-felsic rocks that range from 500-600 Ma (Hibbard et al., 2002). Several arc systems are incorporated into the Carolina Superterrane, including the Hyco Arc (633-612 Ma) and the Albemarle Arc (555-528 Ma) (Hibbard et al., 2013).

The Charlotte Terrane is interpreted to be at least partially related to other PeriGondwanan Terranes, although the aeromagnetic signatures are not congruent (Hatcher et al., 2007). Most of the Charlotte terrain is buried in the subsurface, but the terrane is delineated based on subsurface data. The Charlotte Terrane consists mainly of plutonic rocks, including 530-540 Ma and 570-580 Ma (Butler, 1984; McSween et al., 1991, Hibbard et al., 2002).

The Uchee Terrane lies between Laurentian rocks exposed in the Pine Mountain Window and the Gondwanan Suwannee Terrane (Steltenpohl, 2008). The Uchee Terrane consists of a lower gneiss, metasedimentary rocks, and an upper layer of migmatites and amphibolites (Steltenpohl et al., 2008). The Uchee Terrane is also intruded by plutonic rocks, which are interpreted as Alleghenian (McRae, 1992). These rocks contain 620 to 640 Ma zircon, consistent with an arc terrane origin within the larger Carolina Superterrane (Hibbard et al., 2002). In addition, $300 \mathrm{Ma}$ zircon rims preserve an Alleghanian overprint. No older overprints from the Taconic or Acadian orogenies were dated, indicating that the Uchee Terrane was not connected to North America before the Alleghenian orogeny. Uplift of the Uchee Terrane is estimated to 
have occurred between 276 and $300 \mathrm{Ma}$ based on ${ }^{40} \mathrm{Ar} r^{39} \mathrm{Ar}$ thermochronology, indicating the Uchee Terrane was uplifted during deposition of the Newark Supergroup (Harrison et al., 1985; Mezger and Krogstad, 1997; Steltenpohl et al., 2008).

The Charleston Terrane and Milledgeville Terrane are located to the East of the Uchee Terrane. The Charleston Terrane is completely buried by coastal plain sediments, while the Milledgeville Terrane is exposed at the surface (Hibbard et al., 2002). Both Terranes are poorly constrained and understood, although they are believed to be compossed of rocks metamorphosed during the Alleghanian orogeny. Zircon from a granitoid within the Charleston Terrane yielded a U-Pb age of $600 \mathrm{Ma}$ (Heatherington, 1993).

\section{Gondwana Terranes}

The Suwannee Terrane: Florida basement rocks were initially recognized as exotic to North America by Wilson (1996), and successive studies have supported this interpretation based on geochemical data (e.g., Mueller et al., 2014), and tectonic reconstructions (e.g., Pollock et al., 2012). The exotic crustal fragment that forms the Florida Peninsula is called the Suwannee Terrane, and it accreted onto southeastern Laurentia during the late Paleozoic Alleghanian orogenesis, following the earlier accretion of the Charleston and Carolina terranes (Fig. 60; Hibbard et al., 2002; Mueller et al., 2014). The terrane is thought to have been translocated into its current position by transform movement following accretion (Mueller et al., 2014).

The geology of the Suwannee Terrane is poorly understood, due to the scarcity of wells that penetrate basement rocks. Paleozoic clastic rocks of the Suwanee Terrane are classified as Gondwanan on the basis of fossil assemblages and detrital zircon studies (e.g., Pojeta et al., 1976; Mueller et al., 1994). Zircon signatures are dominated by 515-636 Ma and 1967-2250 Ma 
peaks, which is consistent with Pan-African (525-680 Ma) and Eburnean-Trans Amazonian peaks (1900 Ma-2200 Ma), typical for a Gondwana source (Mueller et al., 1994., Ennih and Liegois., 2008) and similar to the Peri-Gondwanan Terranes (525-680 Ma; Mueller et al., 1994; Heatherington et al., 1999; Heatherington et al., 2003). The lack of any Laurentian signatures suggests that the pre-Mesozoic Suwannee Terrane was not in contact with Laurentia during the Paleozoic (Mueller et al, 2014). 


\section{Basement Geology of Florida}

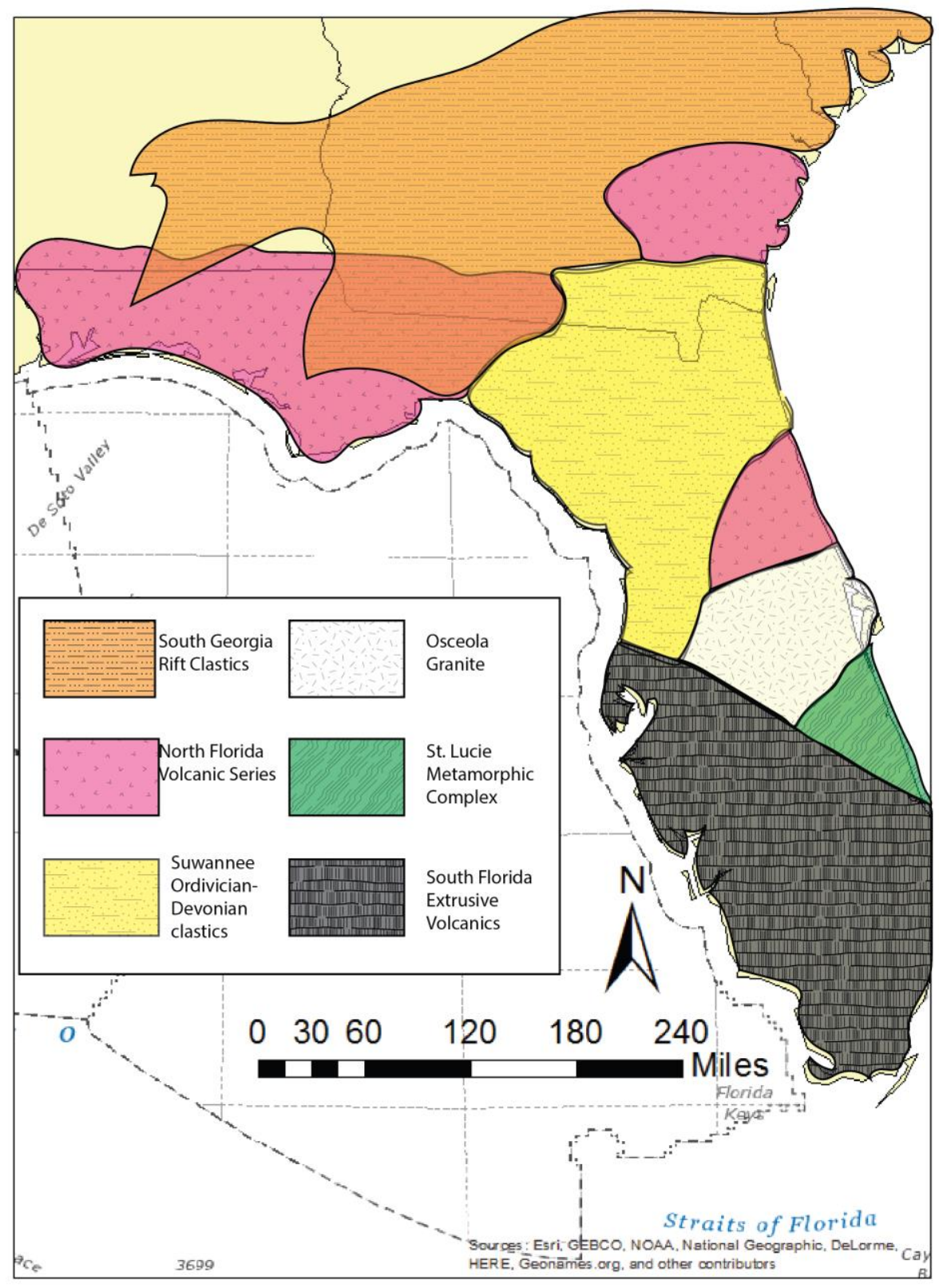

Figure 60. Potential source map for the SGR and SFB of Florida. The SGR is marked above. The SFB rests above the South Florida Extrusive volcanics. Modified from Dallmeyer, (1987) Heatherington et al. (1997); Mueller et al. (2014). 
Volcanic and plutonic rocks are prevalent in the subsurface throughout northern and central Florida (Dallmeyer, 1989; Heatherington and Mueller, 1991; Heatherington and Mueller, 1999). The oldest rocks identified in the Suwannee Terrane is the Osceola Granite Complex (552 Ma; Heatherington and Mueller, 1996), thought to have formed during the Pan-African orogeny in West Africa and the Brasiliano orogeny in South America (Dallmeyer; 1989). The St. Lucie metamorphic complex in central and southern Florida is composed of amphibolite and biotitemuscovite schist (Dallmeyer, 1989), which has been dated at 504-530 Ma by K-Ar and Rb-Sr geochronology (Arthur 1988; Dallmeyer, 1989).

Wiggins Block: The Wiggins Block is a structural arch west of the Suwannee Suture Zone overlain by Jurassic sediment of the Haynesville Formations (Rhodes and Maxwell, 1993; Montgomery, 2000). Cooling ages of 275 to 300 Ma were calculated based on K-Ar dating (Dallmeyer, 1989; Tew et al., 1991) indicate the Wiggins block was uplifted during Alleganian collision and before deposition of Triassic strata in the EGOM (Montgomery, 2000). Lithologies of the Wiggins block include metamorphic schist, quartzite, gneiss, and granite, although the petrology of these rocks is poorly understood since so few wells have penetrated to that depth (Dallmeyer, 1989). Thus, the origin of the Arch is still poorly understood, as is its relation to Laurentia and Gondwana. The Suwannee suture has been interpreted by previous authors to extend north of the Wiggins arch into southern Alabama, and thus the Wiggins arch is likely of Gondwanan origin (Thomas, 2010; Mueller et al., 2014).

Yucatan: The Yucatan peninsula is largely composed of the Maya block (also known as the Yucatan block; Dickinson and Lawton, 2001). Pan-African zircons are fairly common in Yucatan, including the Mississippian Santa Rosa Formation and shocked zircons from the Chicxulub impact crator (Krogh et al., 1993; Weber et al., 2006; Weber et al., 2009). The 
Mississippian to Penyslvanian Santa Rosa Formation contains a mix of Pan-African (560 to 660 Ma), Mesoproterozoic (900 to $1600 \mathrm{Ma}$ ), and Paleozoic ages (318 to $415 \mathrm{Ma}$; Weber et al., 2006; Weber et al., 2009). Mesoproterozoic age likely indicate sourcing from the Oaxacan complex (Weber and Köhler, 1999; Solari et al., 2003), Pan-African ages are likely from Yucatan and Suwannee, and Paleozoic ages are likely derieved from the Maya mountains (Steiner and Walker, 1996).

Northern South America: South America lithosphere is composed largely of cratons, including the Amazonian Craton in northern South America. The Amazonian craton is composed of a series of at least six distinct Archean and Proterozoic tectonic provinces, including Sunsas Belt (950-1280 Ma), the San Ignacio (900-1500 Ma), Rio Negro-Juruena (1550-1780 Ma) Ventuari-Tapajò (1810-1980 Ma), the Maroni-Itacaiunas (2005-2250 Ma), and the Central Amazonian (greater than 2600 Ma; Cordani et al., 2009). The Amazonian Craton is bound to the south by Neoproterozoic and Paleozoic terranes, and to the east by the late Mesozoic-Cenozoic foreland basin sediments and mountains associated with the Andean belt (Cordani et al., 2000). Paleozoic volcanic and metamorphic rocks in South America formed during the Famatinian Orogen (460-480 Ma), which consists of high-grade metamorphic rocks and granites (Salda et al., 1991). The timing of orogenic activity in the Famatinian is comparable to the Taconic in Laurentia, and is a possible extension of the Taconic into South America (Salda et al., 1998). Paleozoic volcanics in South America may have served as a source of sediment during the Triassic/Jurassic into the GOM near Cuba, based on 398 Ma and 452 Ma detrital zircons in the San Cayetano Formation (Rojas-Agramonte et al., 2008).

West Africa: West Africa is an Archean craton bounded to the west by the Pan-African collisional belt, including the Liberian province (2700 Ma; Colver et al., 1991; Ennih and 
Liègeois, 2008). Proterozoic rocks include the Eburnean gneiss, quartzites, and marbles (19002200 Ma; Culver and Hunt., 1991; Schofield and Gillespie, 2007). Pan-African orogeny produced synorogenic magmatic and metamorphic rocks that range in age from 550-650 Ma (Dallmeyer and Villeneuve, 1987).

\section{EGOM Rift Volcanism}

In rift basins throughout Florida/Georgia, tholeiitic basalt, diabase, and tuffs associated with the CAMP volcanism are common. Rift-related volcanism in the Southern Florida Volcanic Series erupted alkali basalts and rhyolites (Heatherington and Mueller, 1991). The basalts of southern Florida are believed to be sourced from a zone of mantle upwelling, which may have driven extension (Heatherington and Mueller, 1991). Basalt ages in southern Florida Rb-Sr range

from 183 to $199 \mathrm{Ma}$, and are similar to the ages of basalts of northern Florida (Heatherington and Mueller, 2003). Diabase from offshore western Florida records a crystallization age at $190 \mathrm{Ma}$ based on Ar-Ar dating (Dallmeyer, 1984). Rhyolite in southern Florida are interpreted to have formed from silica-rich magma, believed to have formed due to intracrustal melting (Heatherington and Mueller, 1991). Rhyolite range in age from 165 to 189 Ma based on Rb-Sr and K-Ar dating (Heatherington and Mueller, 2003).

\section{Provenance Interpretation}

$\underline{\text { South Florida Basin }}$

Age populations for the South Florida Basin are displayed in Figure 61. The offshore ODP sample is excluded from this discussion, as it is not part of the early Mesozoic rift sedimentary sequence. Interpreted source terranes for the detrital zircon ages of samples 778 , 
10566, 3578, 10595, and 2012 samples are displayed in Figure 60 and 61. These SFB samples are dominated by a Gondwanan/Peri-Gondwanan signature (525-680 Ma), which constitutes $76 \%$ of the composite age distribution. Paleoproterozoic zircon grains are interpreted to have originated from Eburnean/Trans-Amazonian terranes; these Paleoproterozoic zircon form the second largest population at $9 \%$ of the composite age distribuion. Grenville-aged zircon grains are not common in the SFB (1\%), nor are Taconic, Acadian and Alleghanian zircon ages (5\%, $\sim 1 \%$ and 2\%, respecitively). Together, these Neoproterozoic and Paleoproterozoic age populations, with a lack of 1,000-1,200 Ma zircon grains, are consistent with sediment derivation from rocks in the Suwanee Terrane. Both of these populations are present in all samples from the South Florida Basin. U/Th ratios consistent with metamorphic zircons around $600 \mathrm{Ma}$, especially common in sample 3578, were likely derived from the St. Lucie Metamorphic Complex in southern Florida. Other potential sources of metamorphic zircon this age include the margin of West Africa and northern South America, which could have sourced the Pan-African and TransAmazonian/Eburnean zircon found in the Suwannee metamorphosed sedimentary rocks. Sourcing from South America is unlikely as this influx would also include Mesoproterozoic ages. The Archean Peak (2\%) likely originated from the West Africa Craton, and recycled zircons from Suwannee metamorphic rocks or Paleozoic clastics. 

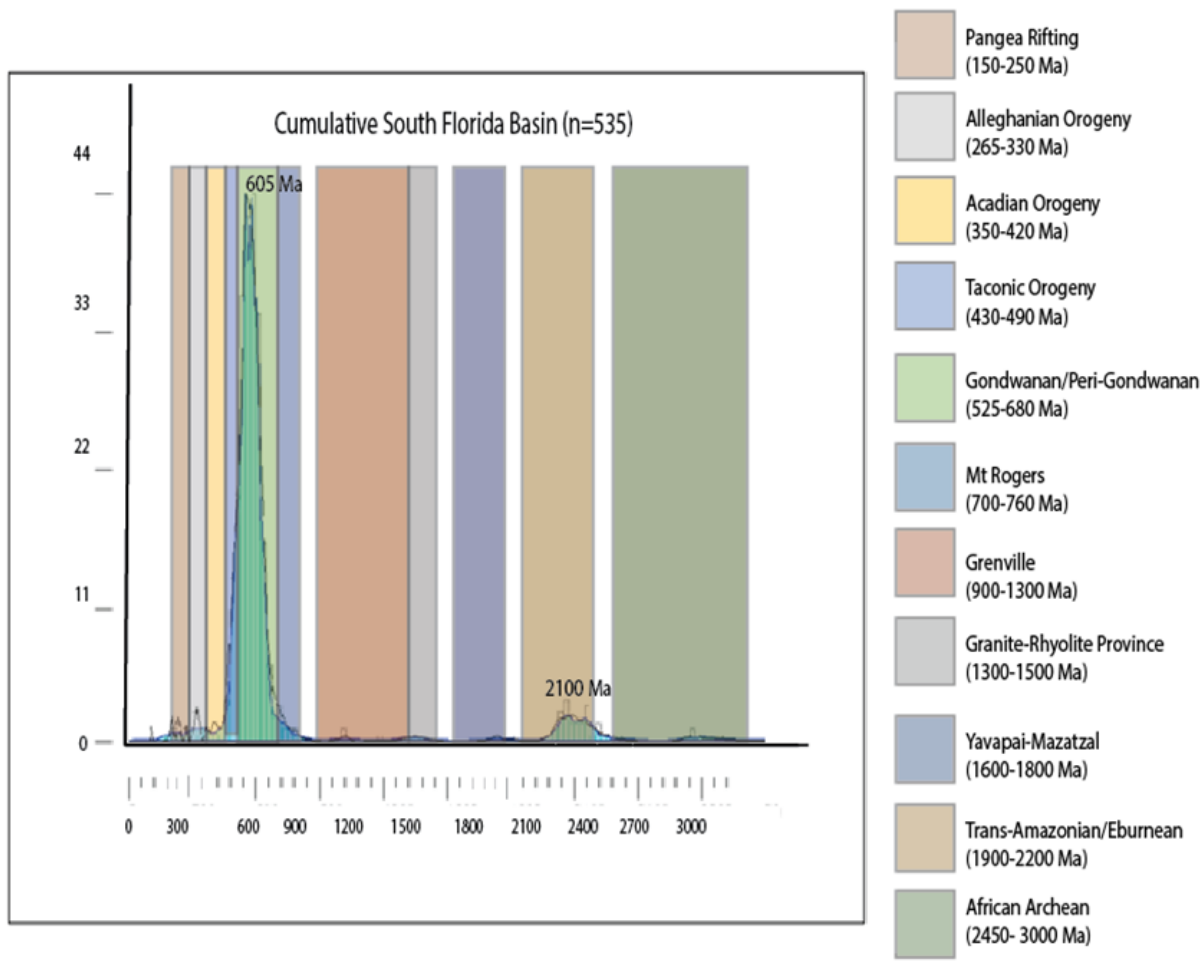

Figure 61. Cumulative plot and interpreted terranes for the SFB.

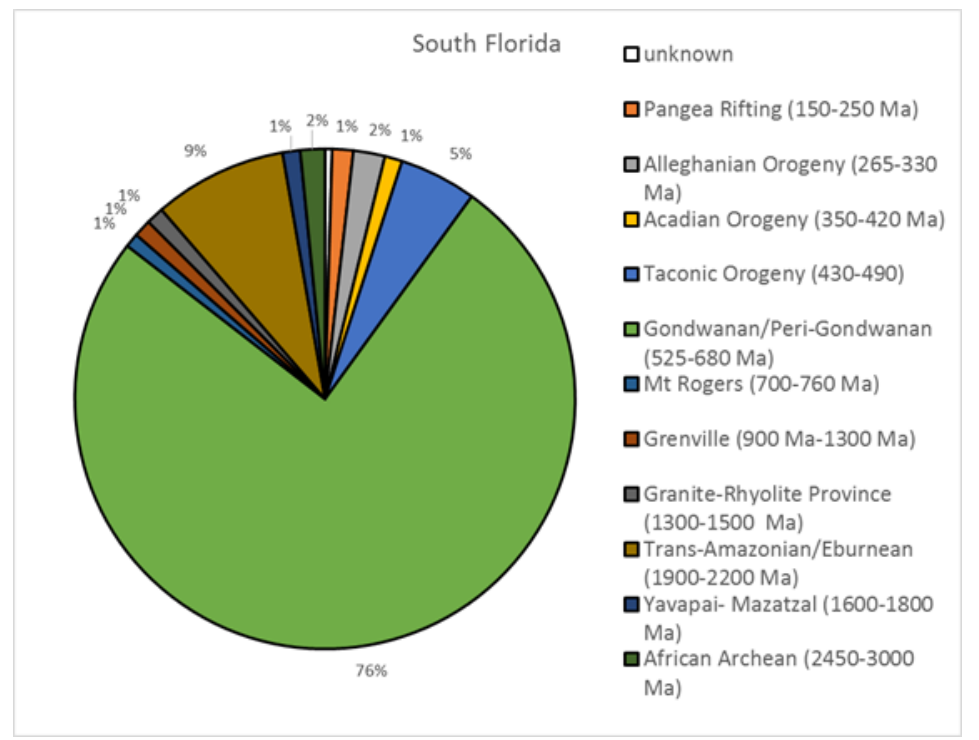

Figure 62. Percentage of each terrane population present in the SFB. The SFB is dominated by a Gondwanan/Peri-Gondwanan Peak (525-680 Ma). 

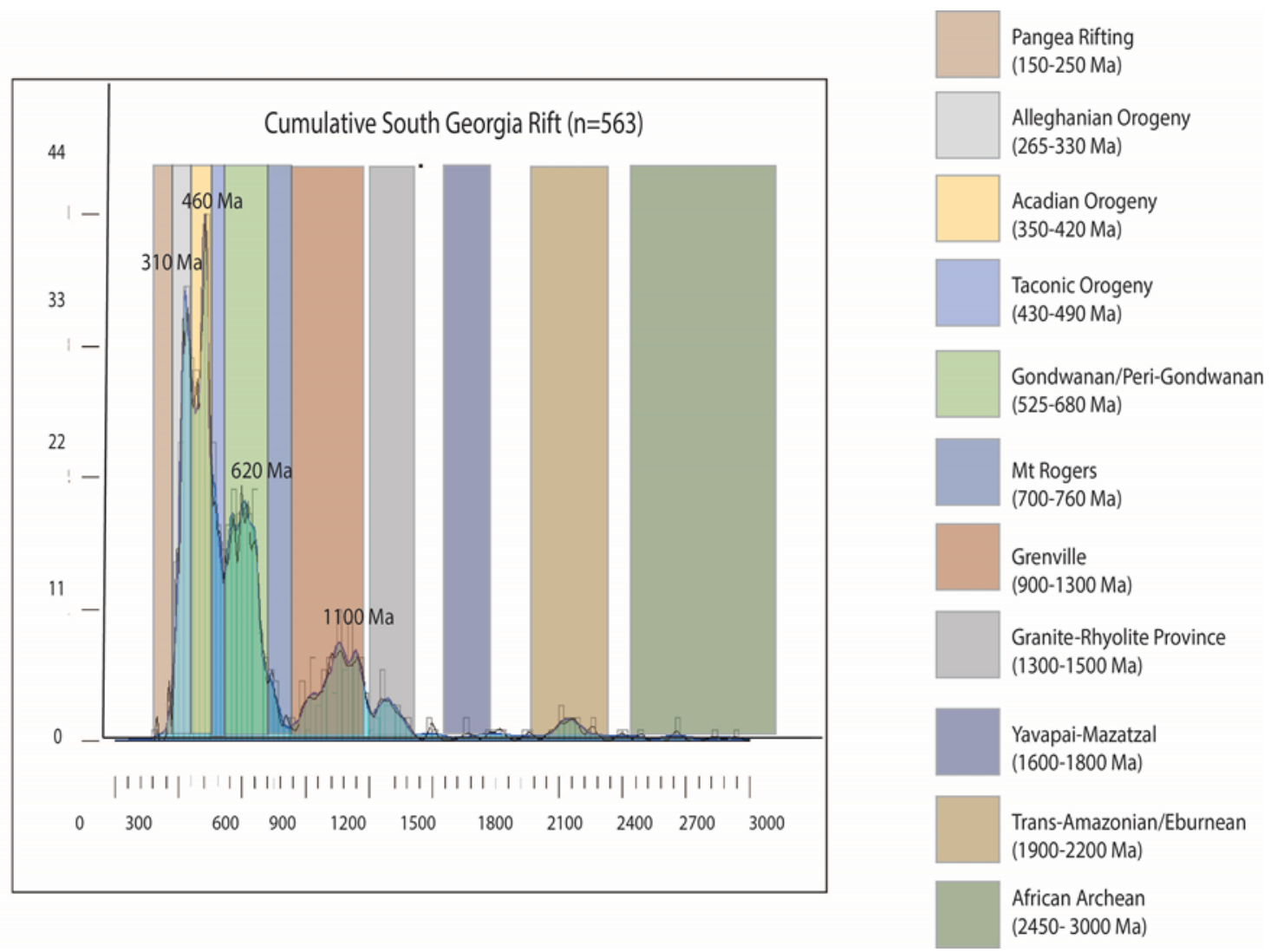

Figure 63. Cumulative plot and interpreted terranes for the SGR.

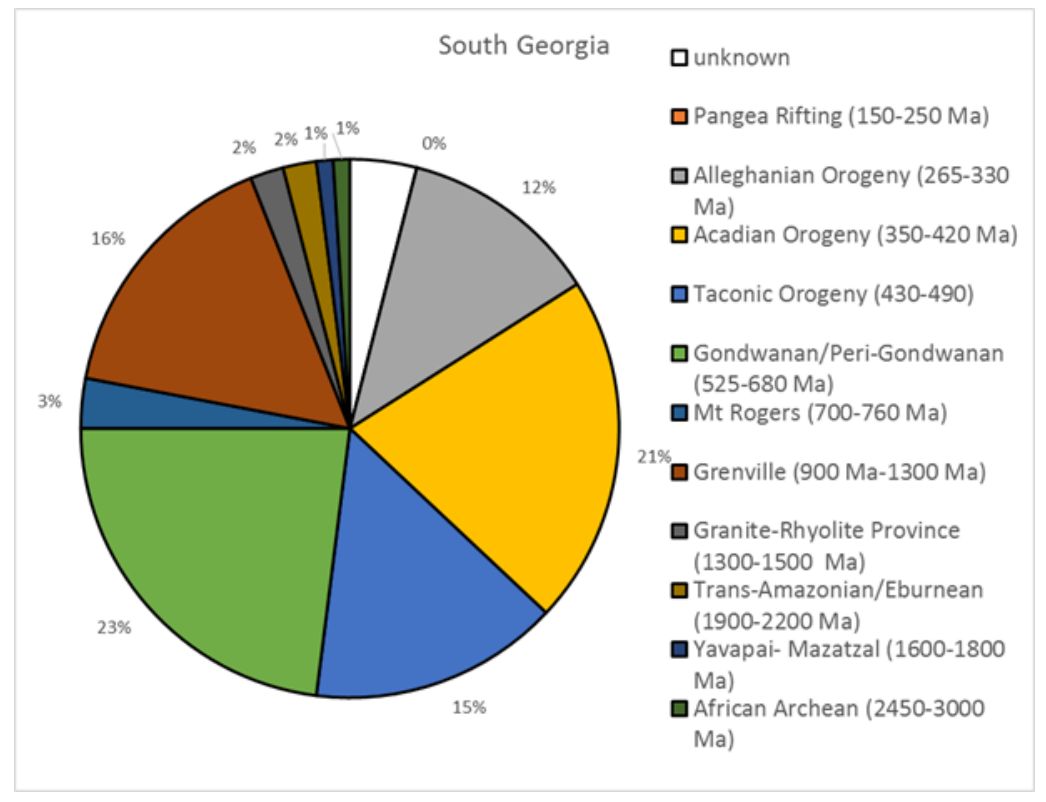

Figure 64. Percentage of each terrane present in the SGR. The SGR contains a mix of Gondwanan and Laurentian Terranes. 
Surprisingly, only a minor peak $(1 \%)$ is consistent with South Florida Volcanics in the SFB associated with rifting of Pangea (150-250 Ma); thus the South Florida Volcanics do not appear to have been major contributors of sediment to the SFB clastics. Maximum depositional age for SFB samples in this study range from 195-237 Ma, which is consistent with South Florida Volcanic age ranges (165 to $199 \mathrm{Ma}$; Heatherington and Mueller, 2003). South Florida ages are slightly younger than the expected 200 Ma CAMP rifting event in Laurentian rift basins, indicating volcanism in the South Florida region may have post-dated rifting in the central Atlantic region. Basalts in southern Florida may have been zircon poor, but silica-rich basalts similar to the basalts in southern Florida can often contain zircon. The South Florida Volcanics may have either been eroded away from uplifted horst blocks, or were deposited in depositional lows and buried by sediment before they could be incised and eroded to serve as a sediment source in the EGOM.

Only $12 \%$ of zircon ages present in the SFB could be associated with Laurentian sources. This includes Alleghenian (265-330 Ma), Acadian (350-420), Taconic (430-490), Mt Rogers (700-760) Grenville (900-1300 Ma), and Yavapai-Mazatzal (1600-1800). None of these ages are prevalent in the Suwannee Terrane. One potential source that may have fed Paleozoic and older detritus is the Maya block, also known as the Yucatan block (Fig. 68). Detrital zircon geochronology of the Upper Carboniferous Santa Rosa Formation from Yucatan by Weber et al. (2006) identified a dominant Pan-African source (540-560 Ma), as well as secondary populations of Paleozoic (421 Ma), Mesoproterozoic (1000-1600 Ma), Paleoproterozoic (1800-2100 Ma) and Archean ages (2600-3100 Ma). Provenance of the Santa Rosa Formation was interpreted to be the Suwannee Terrane and/or West Africa, as well as additional sediments from northern South 
America (Fig. 65). Provenance of the Santa Rosa Formation indicates Late Carboniferous docking of Yucatan with Laurentia during the Alleghenian. Sediment derived from Yucatan was likely shed into the SFB as it was rifted away from the southern margin of Laurentia and West Florida during the opening of the GOM.

Another possible explanation is that at least some sediment derived from southern Laurentia was deposited in the SFB, either by eolian or fluvial input. Similar sediment pathways from the SE Appalachian extended across northern and south Florida during the Oligocene,

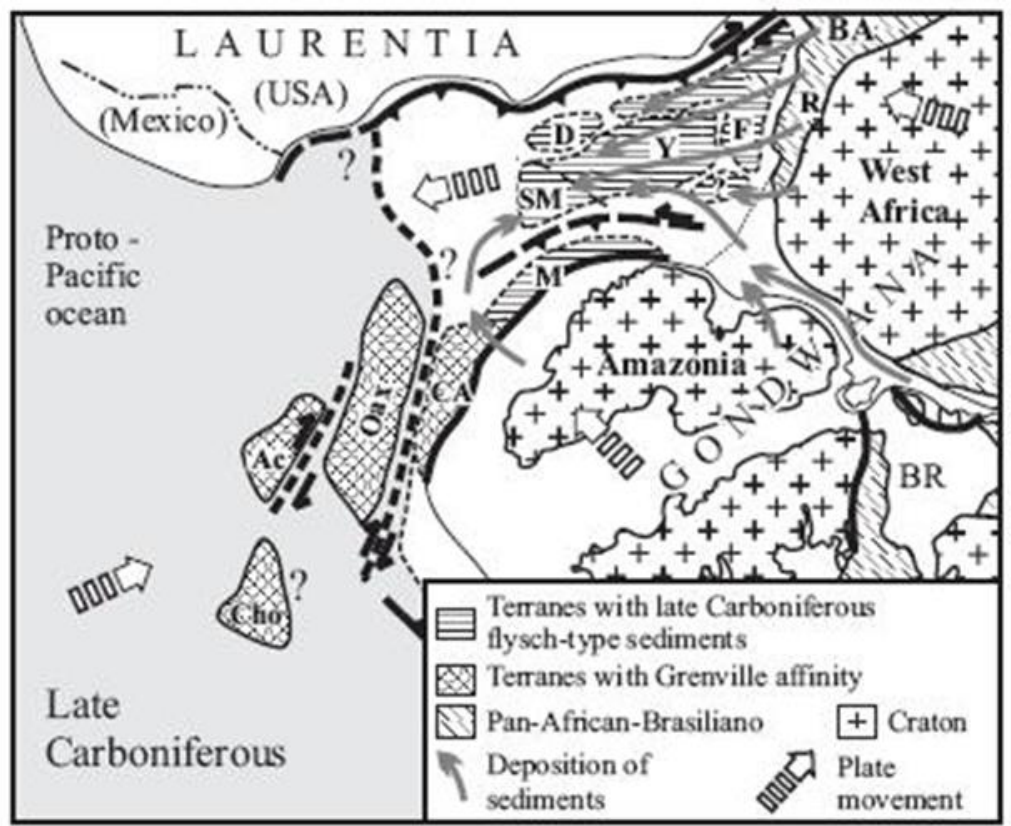

Figure 65. Late Carboniferous provenance model for the Santa Rosa Formation in Western Yucatan. Florida and West Africa served as a dominant source of sediment for the Santa Rosa. From Weber et al. (2006). F-Florida, Y-Yucatan, SM-Southern Maya Block, R-Rokelides, Cho-Chortis block, BR-Brasiliano, ChisChiapas Massif, CA-Colombian Andes, BA-Bassarides-Mauritanides, Ac-Acatlan Complex, D-Delicias Basin, M-Merida Terran. leaving a thin deposit of sand

(Warzeski et al., 1996; Hine, 2009). These deposits are inferred to have been deposited during a marine regression by prograding deltas that extended into southern Florida. This theory is considered controversial, as rivers would have had to flow over topographically high terrain in northern Florida. The Oligocene transport of Appalachian sediment occurred during shoreline regression, which allowed river deltas to prograde into southern Florida. The end of the Triassic was marked by a similar slight fall in sea level (Haq et al., 1987). A similar system of rivers may have existed during the early Mesozoic. 
Interpreted tectonic settings for

the SFB are displayed on the QtFL

diagram in Figure 66. All samples

plotted within the Cratonic

Interior/Transitional Crust source

terrane. Samples are dominated by high

quartz content (50-93\%) and secondary

minor plagioclase, orthoclase,

microcline, and perthite (6-50\%). Lithics

are very uncommon. The cratonic

interior the samples were likely derived

\section{South Florida}

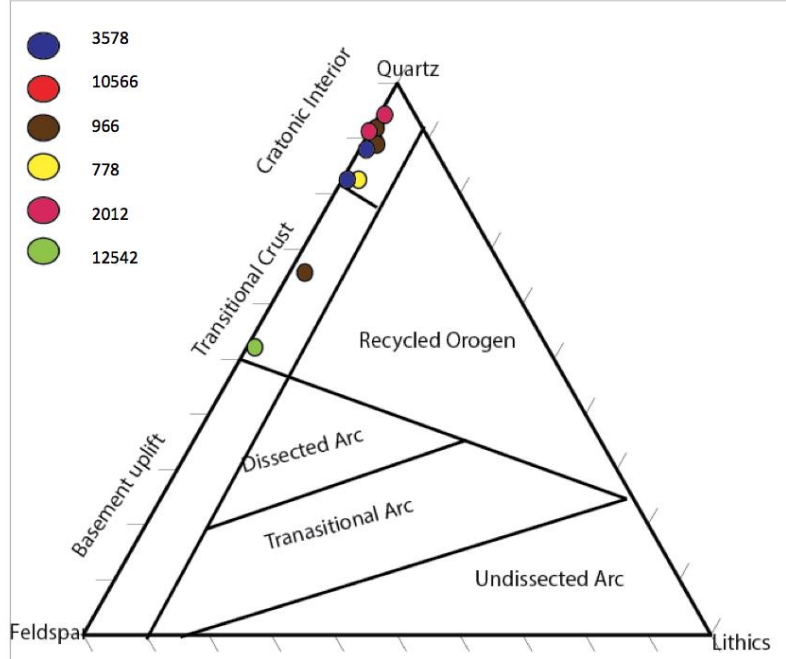

Figure 66. QtFL ternary diagram for the SFB. The majority of the samples are dominated by quartz and feldspar, interpreted as cratonic interior. Perthites are common in select samples. The Wood River was likely derived from Paleozoic cover of the Suwannee Terrane, and the Osceola Granite. Diagram modified from (Dickinson and Suczek, 1979).

from Paleozoic clastics of the Suwannee

Terrane, as well as input from igneous and metamorphic rocks including the Osceola Granite and metamorphic St Lucie Metamorphic complex. Feldspar laths indicate input from volcanic rocks, but the scarcity of volcanic lithic fragments and Mesozoic detrital zircon ages indicate volcanic rocks were not a major source of sand. Sample 1411 is dominated by perthite. Perthite is typically altered very quickly, indicating source area was proximal to the basin. Perthites are common in deeply-exhumed granites, and so perthite grains in SFB sandstones were likely derived from the Osceola Granite of the Suwannee Terrane. Sample 1411 is located far north of other samples in the South Florida Basin, and rests above the Osceola granite, which supports proximal sourcing. 


\section{$\underline{\text { South Georgia Rift }}$}

Population ages for the SGR are displayed in Figure 63 and 67. The SGR contains a mix of Laurentian zircon ages (61\%) and Gondwanan zircon ages (39\%). Acadian-aged zircon form significant populations in the SGR $(\sim 21 \%)$. Around $12 \%$ of zircons from the SGR are consistent with an Alleghenian source. Grenville-aged zircon grains are fairly common in the SGR (16\%). Taconic ages account for $15 \%$ of the SGR samples. Gondwanan/Peri-Gondwanan signatures constitue $23 \%$ of detrital zircon ages from the SGR, considerably less than what was observed in the SFB. Mount Rogers sourcing is not common in the SGR $(\sim 3 \%)$.

The proportion of source terranes differs spatially between SGR samples. Age signatures for each sample are displayed in Figures 61, 63, and 68. All samples contain significant Laurentian zircon age signatures. The SGR samples are dominated by a mix of Alleghenian (265-330 Ma), Acadian (350-420 Ma), Taconic (430-490 Ma), Gondwanan (525-680 Ma), Mt. Rogers (700-760 Ma), Grenville (900-1300 Ma), and Granite Rhyolite (1300-1500 Ma) sources. Together, Laurentian-affinity zircons constitute $73 \%$ of the sample. The Blue Ridge rocks, which extends into northern Georgia, contain abundant Grenville zircons, which may have served as a potential source. Grenville rocks are also exposed in the Pine Mountain Window in western Georgia, although these rocks may not have been exposed during deposition of the Newark (Steltenpohl et al., 2010). The Acadian population is especially abundant (21\%). Acadian orogenic rocks are not abundant in the southern Appalachians. Acadian populations may have been derived from unroofing events that were later sequestered in the SGR. Plutons associated with the Taconic, Acadian, and Alleghenian orogenies are common in the Blue Ridge and multiple Peri-Gondwanan Terranes, including Alleghenian plutons in northern and southern Georgia (Speer et al., 1994). Laurentian age signatures in the South Georgia Rift may have been 
sourced from a combination of long distance transport from Laurentian terranes, recycled zircons from Peri-Gondwanan Terranes, or Appalachian associated plutons. Taconic, Acadian, and Alleghenian-aged zircons are common in the Cat Square Terrane, which may have contributed sediment into the South Georgia Rift. The Alleghanian Clastic Wedge foreland basin sequence in the eastern Appalachians contains Acadian, Taconic, and Alleghanian aged zircons, and likely at least partially sourced the SGR (Thomas et al., 2004).

Gondwanan-derived zircons include Neoproterozoic-early Cambrian ages (525-680 Ma) constituting $23 \%$ of the composite population, and Paleoproterozoic ages (1,900-2,200 Ma) constituting $2 \%$ of the composit population (Fig. 64 and 69). The mix of Laurentian ages indicates transport from the north, and so supports derivation from Peri-Gondwanan Terranes along eastern Laurentia. However, it is possible that these Gondwanan zircon grains originated from the Suwannee Terrane as well. The 1,900-2,200 Ma Trans-Amazonian/Eburnean population is more common in Suwannee rocks than the Peri-Gondwanan Terranes. Because this population in the SGR is minor, it suggests that while it is possible that some sediment was contributed from the Suwannee Terrane, this contribution was less than the influx from northern source areas.

There is great variation in proportion of Laurentian versus Peri-Gondwanan/Gondwanan input across the SGR. Samples 1854 (15\%) and 3113 (35\%) located in the Florida Panhandle and near the Georgia/Florida border, respectively, contain significantly less Appalachian sources than other samples (60\% for 108, 50\% for sample 619, 55\% for 3137, 52\% for 442). Samples 1854 and 3113 are dominated by Neoproterozoic-early Cambrian ages (525-680 Ma). Sample 1854 contains $42 \%$ Neoproterozoic-early Cambrian ages (525-680 Ma) and 5\% Paleoproterozoic (1,900-2,200 Ma) ages, whereas sample 3113 is composed of $25 \%$ Neoproterozoic-early 
Cambrian ages (525-680 Ma), and 3\% Paleoproterozoic (1,900-2,200 Ma) ages. The lack of Appalachian age signatures and presence of Neoproterozoic-early Cambrian ages (525-680 Ma), and 3\% Paleoproterozoic (1,900-2,200 Ma) age, consistent with zircon ages known in the Suwannee Terrane, indicates that both of these samples were likely deposited on the Suwannee side of the Suwannee suture at or near the Suwannee Suture within the Tallahassee Graben. In contrast, samples 3137, 108, and 619 are dominated by Appalachian signatures, and were likely deposited on the Laurentia side of the suture. Neoproterozoic-early Cambrian detrital zircons could reflect Gondwanan Suwannee Terrane input and/or Peri-Gondwanan Terrane input. The occurrence of Neoproterozoic-early Cambrian detrital zircons with Appalachian-aged detrital zircon implies a northern source area that suggests at least partial sourcing from Peri-Gondwanan terranes was likely. Sample 442 contains a relatively large (27\%) Neoproterozoic-early Cambrian age signature along with a significant (52\%) Taconic, Acadian, and Alleghanian age signature, indicating derivation from Appalachian and Gondwanan sources. Grenville peaks (7\%) and Paleoproterzoic peaks (1\%) are both minor compared to other samples. Its location in the northern and western SGR suggests it may have received sediment from both Laurentia and Peri-Gondwanan Terranes.

Sample 3113 from the southern part of the SGR has a large Pan-African zircon age population, but insignificant Appalachian zircon age populations, implying the majority of sediment was derived from the Suwannee Terrane. Only samples 1854 from the Tallahassee Graben and 3113 from the southern SGR contain significant quantities of Paleoproterozoic ages (1,900-2,200 Ma), interpreted to represent a Trans-Amazonian/Eburnean signature. U/Th ratios suggest metamorphic origin for 600 Ma zircon grains, which were likely derived from metamorphic Peri-Gondwanan Terranes or recycled from younger clastic wedges. 
South Georgia Rift Source Terrane Abundance

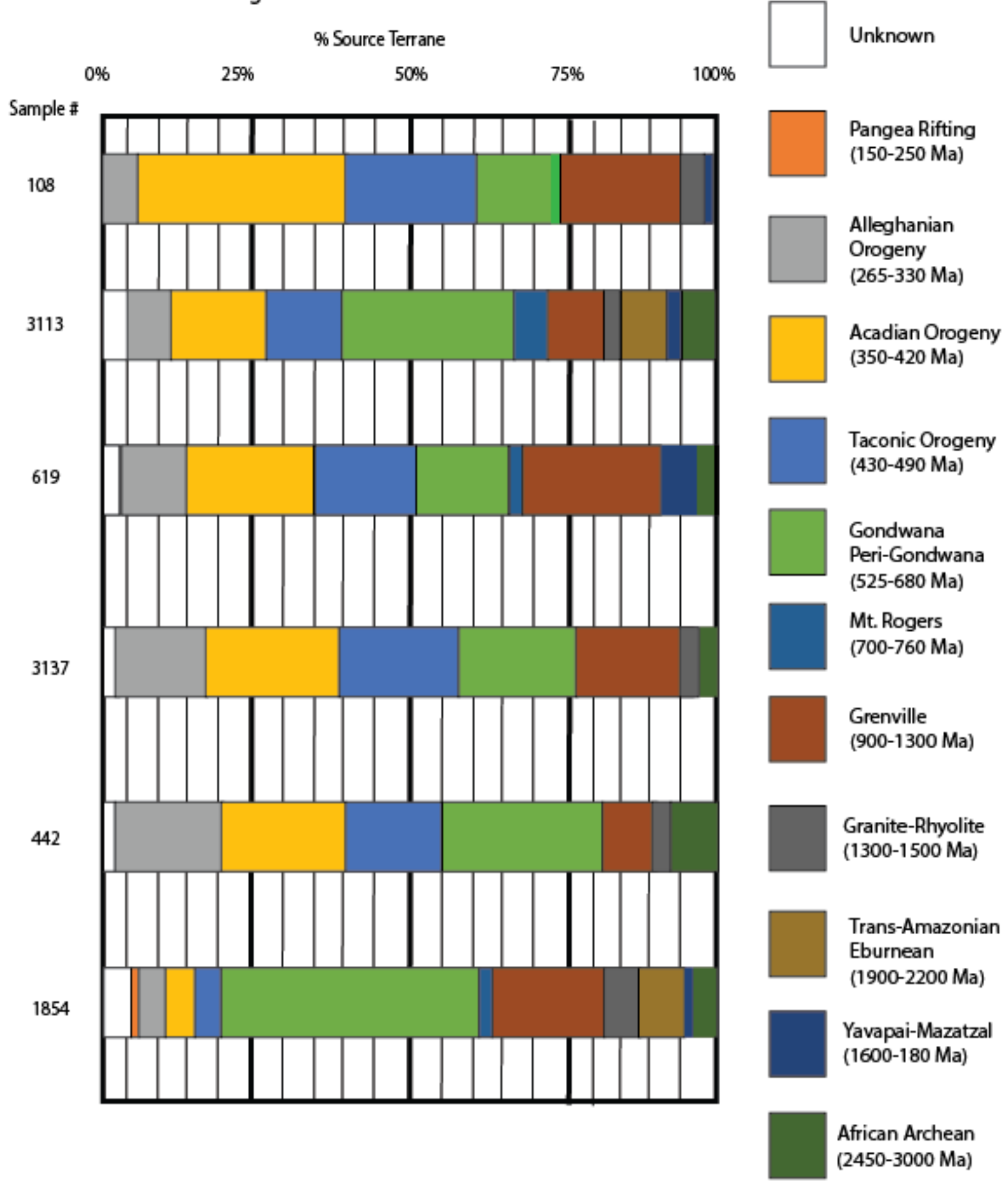

Figure 67. Relative proportion of terrane sourcing for each sample from the SGR. 
No samples from the South Georgia Basin were coarse enough to be point counted, but samples collected for thin sections were dominated by shale, with a mixed feldspar and quartz composition, and very few lithics identified. The high amount of mica fragments is consistent with a metamorphic source lithology, which based on detrital zircon ages indicate metamorphic rocks in Peri-Gondwanan Terranes. Previous point counting interpretions from the SGR in South Carolina from Rine et al. (2014) interpreted a Peri-Gondwanan source based on the abundance of metamorphic minerals and lithic fragments. Based on the U/Th results there are not a significant number of Metamorphic zircon, aside from samples 442, and 3137.

\section{Data Comparison with other Studies}

Thin Section observations are supported by previous provenance studies in the EGOM (Figure 68). Lisi (2013) noticed a similar dominance of quartz in Norphlet samples in the Florida Panhandle and offshore western Florida, which was attributed to input from dominantly Gondwanan clastic and granitic rocks. Norphlet Formation samples from the Destin Dome and Florida Panhandle contain abundant feldspar, interpreted to have been derived from Laurentia metamorphic and igneous sources. Bovay et al. (2014) also noticed a similar trend in early Cretaceous clastic rocks in offshore western Florida, and they proposed that Gondwanan source areas in the Peninsular Arch are dominated by shales interbedded with fine-grained sandstones, while Appalachian source areas contain predominantly fine-grained micaceous sandstone interbedded with shale. They interpreted Gondwanan sources to be primarily from Paleozoic sandstones and Precambrian granites, based on the presence of fine to coarse grained pure sandstones, while the Cretaceous clastic rocks of the Peninsular Arch were sourced primarily from Peri-Gondwanan Terranes and sources exposed in the Appalachian mountains, based on the 
presence of micaceous sandstones. They proposed that the Peninsular Arch of Florida served as a dominant source of sediment into the EGOM, with sediment transportation to the west.

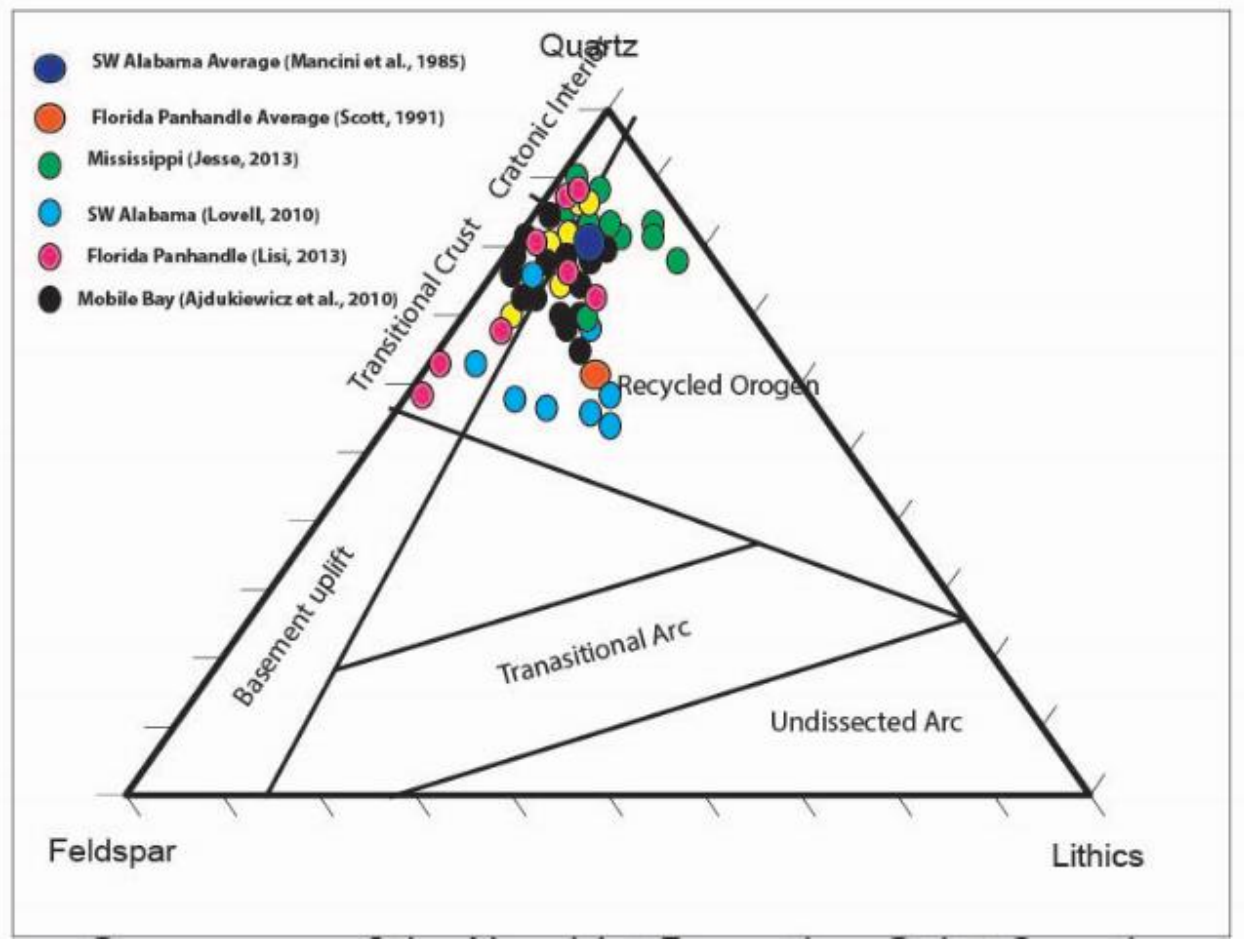

Figure 68. Provenance of the Norphlet Formation in the EGOM from previous studies. The Norphlet is dominated by Cratonic Interior, Transitional Crust, and Recycled Orogen sources.

Zircon data collected during this study was compared to previous studies by Lovell (2010), Lisi (2013), and Jesse and Weislogel (2014) of the Norphlet in the EGOM to determine if the SGR, SF, and Norphlet in the EGOM may share similar sources. If so, this could support the conclusion that both basins may have received sediment routed through similar pathways into the EGOM. Based on similarities between zircon provenance and paleogeographic location, 8 major sample groupings were identified (Table 12). The K-S test was used to identify statistical similarities between the sample groupings (Table 13). A CDP for the 8 groupings is displayed in 
Figure 69. Major differences are noted 530 and 1000 Ma. An overview of the major groupings and the implications of provenance are described below.

\begin{tabular}{|c|c|c|c|c|}
\hline Population & Samples & Major Age Peaks & Sources & Youngest Age \\
\hline West Florida & $\begin{array}{l}382,383,2523 \\
2516,2012\end{array}$ & $559 \mathrm{Ma}, 637 \mathrm{Ma}$ & $\begin{array}{l}\text { Suwannee } \\
\text { Terrane }\end{array}$ & $456 \mathrm{Ma}$ \\
\hline South Florida & $\begin{array}{l}2012,3578, \\
10566,15095, \\
778, \text { ODP }\end{array}$ & $568 \mathrm{Ma}$ & $\begin{array}{l}\text { Suwannee } \\
\text { Terrane }\end{array}$ & 108/105 Ma \\
\hline $\begin{array}{l}\text { Apalachicola } \\
\text { Embayment }\end{array}$ & $\begin{array}{l}6406,6396,1623, \\
563\end{array}$ & $\begin{array}{l}294 \mathrm{Ma}, 430 \mathrm{Ma} \text {, } \\
560 \mathrm{Ma}, 617 \mathrm{Ma}\end{array}$ & $\begin{array}{l}\text { Alleghenian, } \\
\text { Taconic, (peri)- } \\
\text { Gondwanan } \\
\text { Terranes, } \\
\text { Grenville }\end{array}$ & $198 \mathrm{Ma}$ \\
\hline Florida Panhandle & $\begin{array}{l}1004,1096,1196 \\
1193\end{array}$ & $\begin{array}{l}316 \mathrm{Ma}, 392 \mathrm{Ma}, \\
424 \mathrm{Ma}, 583 \mathrm{Ma} \text {, } \\
1064 \mathrm{Ma}\end{array}$ & $\begin{array}{l}\text { Alleghanian, } \\
\text { Acadian, Taconic, } \\
\text { (peri) Gondwanan } \\
\text { Terranes, } \\
\text { Grenville }\end{array}$ & $284 \mathrm{Ma}$ \\
\hline $\begin{array}{l}\text { Southern } \\
\text { Alabama }\end{array}$ & $\begin{array}{l}5115,1036, M-6, \\
949,6805,1552 \\
1543,5115,860 \\
6848\end{array}$ & $\begin{array}{l}298 \mathrm{Ma}, 336 \mathrm{Ma} \text {, } \\
435 \mathrm{Ma}, 561 \mathrm{Ma} \text {, } \\
629 \mathrm{Ma}, 1088 \mathrm{Ma}\end{array}$ & $\begin{array}{l}\text { Acadian, Taconic, } \\
\text { Pan-African, } \\
\text { Grenville }\end{array}$ & $201 \mathrm{Ma}$ \\
\hline $\begin{array}{l}\text { South Georgia Rift } \\
\text { northern subbasin }\end{array}$ & $\begin{array}{l}108,619,3137, \\
442\end{array}$ & $\begin{array}{l}332 \mathrm{Ma}, 432 \mathrm{Ma} \\
584 \mathrm{Ma}, 1077 \mathrm{Ma}\end{array}$ & $\begin{array}{l}\text { Acadian, Taconic, } \\
\text { Pan-African, } \\
\text { Grenville }\end{array}$ & $250 \mathrm{Ma}$ \\
\hline $\begin{array}{l}\text { South Georgia Rift } \\
\text { southern subbasin }\end{array}$ & 1854,3113 & $\begin{array}{l}345 \mathrm{Ma}, 430 \mathrm{Ma} \text {, } \\
569 \mathrm{Ma}, 602 \mathrm{Ma} \text {, } \\
640 \mathrm{Ma}\end{array}$ & $\begin{array}{l}\text { Acadian, Taconic, } \\
\text { Pan-African }\end{array}$ & $197 \mathrm{Ma}$ \\
\hline $\begin{array}{r}\text { Mississippi } \\
\text { Interior Salt Basin }\end{array}$ & $\begin{array}{l}\text { DZ 1, DZ 2, DZ 3, } \\
\text { DZ 4, DZ 5, DZ } 6\end{array}$ & $\begin{array}{l}360 \mathrm{Ma}, 427 \mathrm{Ma}, \\
605 \mathrm{Ma}, 1068 \mathrm{Ma}, \\
1151 \mathrm{Ma}, 1477 \\
\mathrm{Ma}\end{array}$ & $\begin{array}{l}\text { Acadian, Taconic, } \\
\text { Pan-African, } \\
\text { Grenville, Granite- } \\
\text { Rhyolite }\end{array}$ & $204 \mathrm{Ma}$ \\
\hline
\end{tabular}




\begin{tabular}{|c|c|c|c|c|c|c|c|c|}
\hline & $\begin{array}{l}\text { West Florida } \\
4 \text { samples }\end{array}$ & $\begin{array}{l}\text { South Florida } \\
\text { Basin } \\
6 \text { samples }\end{array}$ & $\begin{array}{l}\begin{array}{l}\text { Norphlet } \\
\text { Apalachicola } \\
\text { Embayment }\end{array} \\
4 \text { samples }\end{array}$ & $\begin{array}{l}\text { South Georgia } \\
\text { Rift South } \\
2 \text { samples }\end{array}$ & $\begin{array}{c}\begin{array}{c}\text { Norphlet } \\
\text { Southern } \\
\text { Alabama }\end{array} \\
10 \text { samples }\end{array}$ & $\begin{array}{l}\begin{array}{l}\text { Norphlet } \\
\text { Florida } \\
\text { Panhandle }\end{array} \\
\text { 4 samples }\end{array}$ & $\begin{array}{l}\begin{array}{l}\text { South } \\
\text { Georgia } \\
\text { Rift North }\end{array} \\
4 \text { samples }\end{array}$ & $\begin{array}{l}\text { Mississippi } \\
\text { Interior Salt } \\
\text { Basin } \\
6 \text { samples }\end{array}$ \\
\hline \multirow[t]{8}{*}{ West Florida } & $0 \%$ & $12.5 \%$ & $12.5 \%$ & $4.12 \%$ & $0 \%$ & $8.3 \%$ & $0 \%$ & $0 \%$ \\
\hline & South Florida & $53 \%$ & $20.8 \%$ & $10 \%$ & $0 \%$ & $0 \%$ & $0 \%$ & $0 \%$ \\
\hline & & $\begin{array}{l}\text { Norphlet } \\
\text { Apalachicola } \\
\text { Embayment }\end{array}$ & $83.33 \%$ & $75 \%$ & $8.3 \%$ & $25 \%$ & $6.25 \%$ & $0 \%$ \\
\hline & & & $\begin{array}{l}\text { South Georgia Rift } \\
\text { South }\end{array}$ & $50 \%$ & $0 \%$ & $16.67 \%$ & $20 \%$ & $10 \%$ \\
\hline & & & & $\begin{array}{l}\text { Norphlet } \\
\text { Southern } \\
\text { Alabama }\end{array}$ & $85.67 \%$ & $70.83 \%$ & $0 \%$ & $91.7 \%$ \\
\hline & & & & & $\begin{array}{l}\text { Norphlet Florida } \\
\text { Panhandle }\end{array}$ & $66.67 \%$ & 0 & $69.44 \%$ \\
\hline & & & & & & $\begin{array}{l}\text { South Georgia } \\
\text { Rift North }\end{array}$ & $87.5 \%$ & $0 \%$ \\
\hline & & & & & & & $\begin{array}{l}\text { Norphlet } \\
\text { Mississippi } \\
\text { Interior } \\
\text { Salt Basin }\end{array}$ & $86.67 \%$ \\
\hline
\end{tabular}

Table 13. Major groupings identified, and statistical similarity between each grouping. The percentage indicates how many pairings passed the $K$-S test ( $p>$.05). A higher percentage indicates a higher likelihood for a shared source.

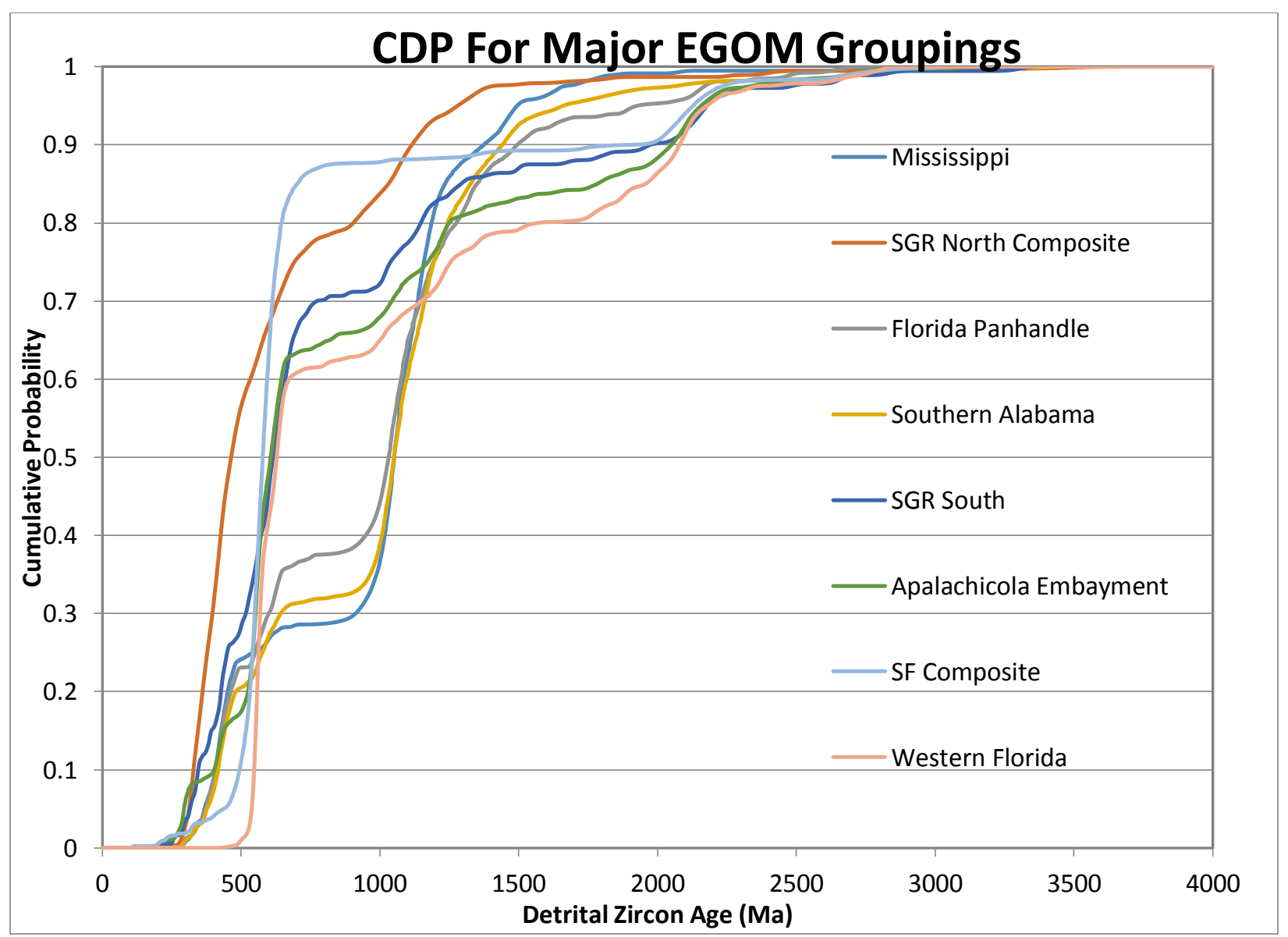

Figure 69. CDP for the 8 major groupings identified in this study. Major groupings are present around $530 \mathrm{Ma}$ and $1000 \mathrm{Ma}$. 


\section{Regional Provenance Model}

Based on the K-S test, Cumulative Probability Density Plots, Normalized Probability Density Plots, and provenance studies in Lovell, (2010), Lisi, (2013) and Jesse and Weislogel (2014), eight major depocenters were identified between samples. A composite CDP of detrital zicron ages for each region is displayed in Figure 69. A Normalized PDP for all groupings is displayed in Figure 71, and a potential provenance model in Figure 72.

\section{Offshore Western Florida (Haynesville)}

Offshore Western Florida samples (382, 383, 2523, 2516) analyzed by Lisi (2013) were collected from interpreted Haynesville and basement samples. Samples were collected from the Tampa Embayment. Samples are derived from primarily Pan-African sources, with two age populations between 580 and 680 Ma. Minor populations from 800-2800 Ma are also common but not prevalent. Rocks of the Suwannee Terrane uplifted in the Peninsular Arch were likely a dominant source for West Florida.

Sample 2012 from this study was collected from central, onshore Florida, from the Suwannee Basin. Sample 2012 rests above diabase 190 Ma (Heatherington and Mueller, 2003). Sample 2012 is interpreted to lie within the Paleozoic clastic fill (Ordivician-Devonian) of the Suwannee Basin (e.g, Heatherington and Mueller, 1994; Boote and Knapp, 2016). Detrital zircon ages of 234 to $324 \mathrm{Ma}$ imply that the clastics are Triassic/Jurassic, and may indicate Triassic syn-rift clastic deposition. Sample 2012 zircon ages are similar to the Tampa Embayment samples from Lisi (2013). 


\section{South Florida Basin (Wood River)}

The SFB provenance results (samples $15095,778,3578,10566$; this study) are from the Wood River Formation, and zircon ages are significantly different from the other age populations in EGOM region, aside from offshore Tampa Embayment samples from Lisi (2013). The SFB is dominated by one population between 480-720 Ma, which is interpreted as predominantly PanAfrican. A population between 2000 to $2200 \mathrm{Ma}$ is also present, which is intepreted as Eburnean/Trans-Amazonian. Provenance for the SFB is intepreted as Suwannee Terrane rocks based on the abundant and overall unimodal Neoproterozoic zircon signatures. Smaller populations around $200 \mathrm{Ma}$ have been attributed to South Florida volcanics, and smaller populations between 300-400 Ma are possibly related to minimal input from Appalachian sources or Yucatan. The ODP sample indicates detrital zircon in the SFB essentially reflect reworking of underlying Suwannee Terrane basement rocks.

Apalachicola Embayment (Norphlet)

The Apalachicola Embayment (563, 162, 6396, 6406; Lovell 2010; Lisi, 2013) is the most similar to the SFB composite. Populations between 520-680 Ma, and 1900-2300 Ma are interpreted as a large Pan-African population and Eburnean population similar to the South Florida Compositie. Appalachian (240-500 Ma) and Grenville (920-1280 Ma) populations are present in the Apalachicola Embayment. The Apalachicola Embayment likely received input from a mix of Suwannee and Laurentian sources, which was initially proposed by Lisi (2013). The Apalachicola Embayment was likely proximal to the Suwannee Suture, similar to samples 1854 and 3113 as proposed earlier. 


\section{Southern South Georgia Rift (Newark)}

The southern SGR samples analyzed (1854, and 3137; this study) have a large population at 500-680 Ma, consistant with Pan-African sources, and sharp populations between 340-460 Ma typical with an Appalachian sources. The southern SGR contains a very small population consistant with an Eburnean/Trans-Amazonian population. The southern SGR was likely derived from Peri-Gondwnan and Suwannee sourcess.

Similarities between offshore Destin Dome Norphlet zircon data from Lovell (2010) and Lisi (2013) with samples from the Talhassee Graben and southern South Georgia Rift supports the interpretation that they may share a similar source. Sample 1854 and 3113 of the SFB are similar to the Apalachicola Embayment composite, as both have a large age peaks associated with Pan-African and Appalachian source, and the composite distributions pass the K-S test. Therefore, it is likely that either: 1) Both western Florida panhandle and Tallahassee Graben/southern portion of the South Georgia Rift share a similar source with the Norphlet in the Apalachicola Embayment and/or, 2) The Tallahassee Graben and southern portion of the South Georgia Rift may have served as a sediment pathway into the EGOM. The Pan-African age population is present all samples throught the Florida EGOM, and was likely sourced from Suwannee Terrane rocks uplifted in the Peninsular Arch.

\section{Southern Alabama Compositie (Norphlet)}

The Southern Alabama Composite (5115, 1552, 1193, 4557, 860, 949, 1196, M6, 162, 10361, 6805; Lovell, 2010 and Lisi, 2013) marks a shift from predominantely Suwanee Terrane age popluations, to predominantly Laurentian age populations, as initially proposed by Lisi (2013). The southern Alabama composite does not have a significant Pan-African (520-680 Ma) 
age population and Eburnean ages are absent. Instead, Appalachian (320-500 Ma) age populations are more abundant than compared to the Apalachicola and SFB composite age distribution. There is a very large population (900-1300 Ma) in southern Alabama, which is attributed to Grenville. In contrast, the SFB and Appalachiacola embayment composite distribution contains very few Grenville-aged zircon. The southern Alabama composite was likely dominated by Laurentian and Peri-Gondwanan sources. The lack of Eburnean/TransAmazonian populations indicates that a Suwannee source was unlikely.

\section{Florida Panhandle (Norphlet)}

The Florida Panhandle composite (1004, 1096, 1080; Lisi, 2013) has large populations attributed to Appalachian (300-500 Ma), Pan-African (540-680 Ma), and Grenville (900-1300 Ma). It also has a small population (2100-2200 Ma) that may be consistant with a Eburnean/Trans-Amazonian population. The Florida Panhandle deposits likely contains sediment from a mix of Laurentian Peri-Gondwanan and Suwannee sources.

\section{Northern South Georgia Rift (Norphlet)}

Northern samples from the SGR $(108,3137,442$, and 619; this study) contain a large population of 320-490 Ma zircon, consistant with Appalachian sources, as well as a second population of 550-680 Ma zircon associated with a Pan-African source. The northern SGR deposits were likely derived from a mix of Laurentian and Peri-Gondwanan/Suwannee Terrane sources. 


\section{Mississippian Interior Salt Basin (Norphlet)}

The Mississippian Interior Salt Basin (MISB) is located in southern Mississippi (samples DZ1, DZ2, DZ3, DZ4, DZ5, DZ6; Jessee and Weislogel, 2014). Samples in the MISB are dominated by Grenville (900-1300 Ma) zircons, as well as Taconic, Acadian, and Alleghanian sources (300-520 Ma). Pan-African Gondwanan zircons are rare in these deposits. 


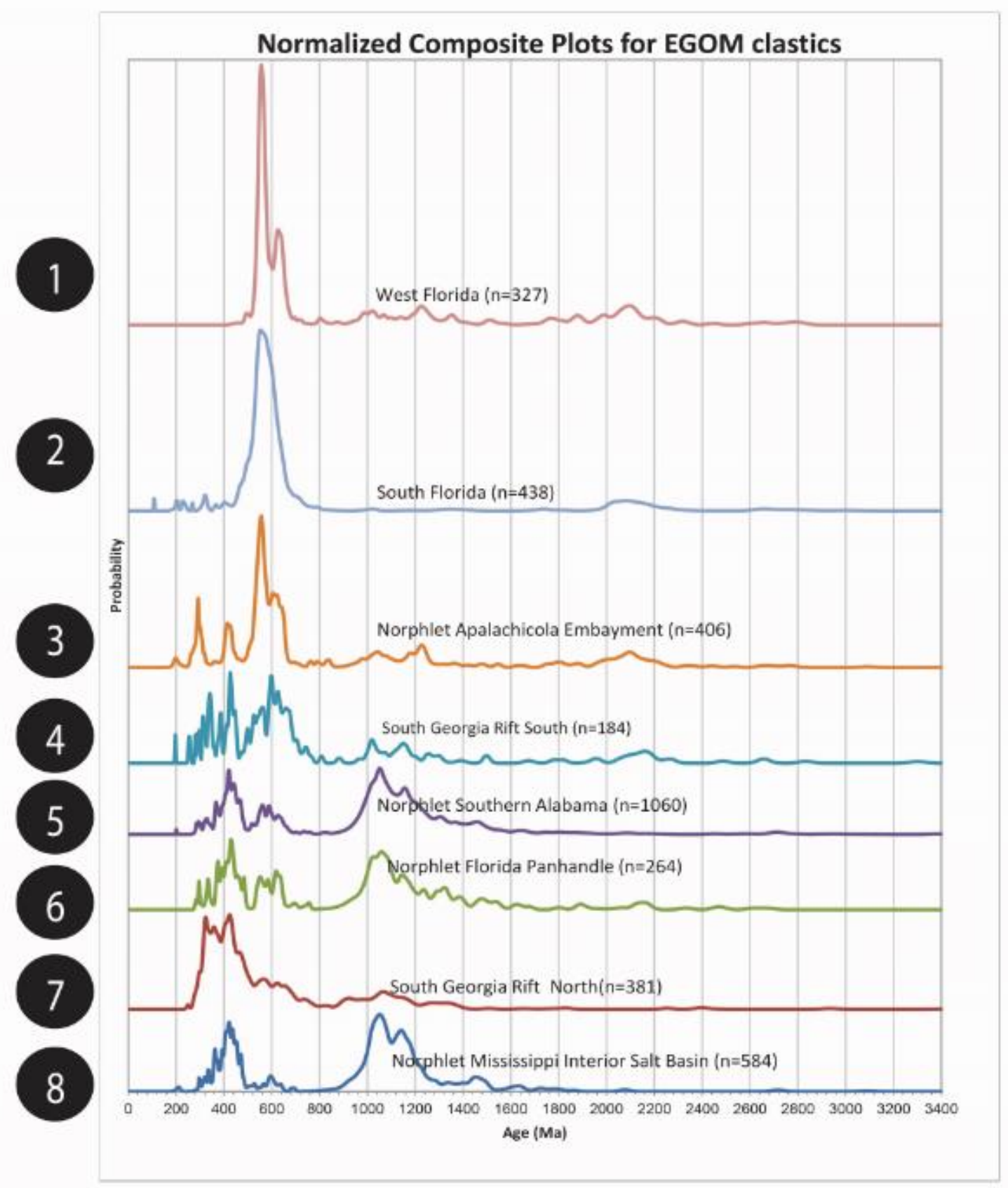

Figure 70. Composite Normalized PDP for the Mesozoic EGOM. Results from this study, Lovell (2010), Lisi (2013) and Jesse and Weislogel (2014). 


\section{Provenance Model}

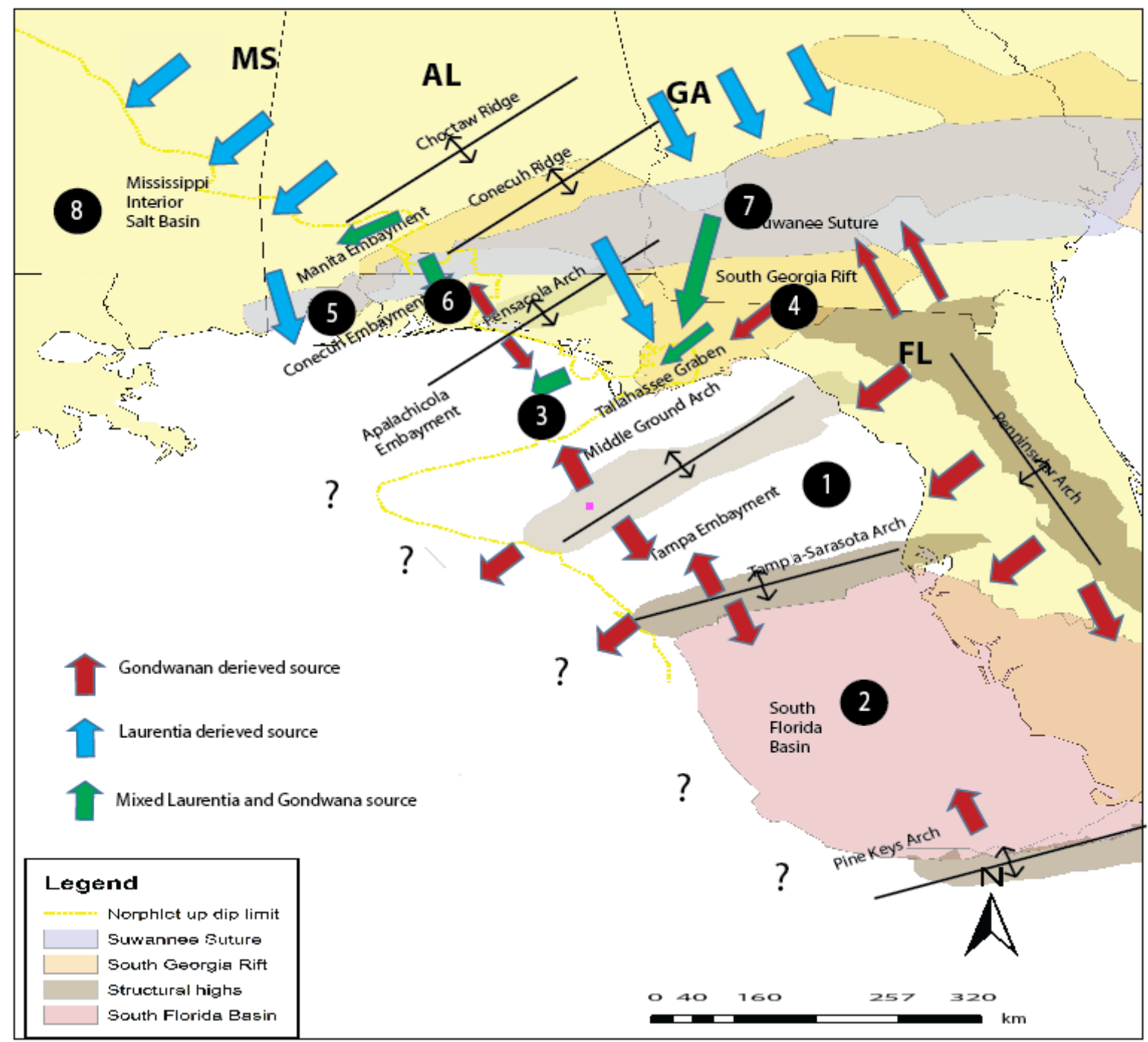

Figure 71. Provenance model for the Norphlet, Haynesville, Wood River, and Newark in the EGOM from this study. Gondwana, Laurentia, and mixed sources are identified. The 8 groupings identified are listed in Figure 71. 


\section{Paleogeographic Interpretations and Regional Provenance Model}

\section{$\underline{\text { South Florida Basin }}$}

Detrital zircon geochronology, thin-sections, and well logs interpretations are used to reconstruct early Mesozoic EGOM paleogeography and provenance. The Peninsular Arch, a structural feature of controversial origin, was likely a paleotopographic high in the middle of Florida throughout the Mesozoic. Sediment shed from this arch was distributed across the SFB and out into the Tampa Embayment, providing texturally immature detritus containing the Neoproterozoic-early Cambrian detrital zircon and quartzose sand along with perthite grains. Provenance studies of Valanginian strata in the northern EGOM indicates that the Peninsular arch was a source of sediment until at least the Lower Cretaceous (Bovay et al., 2014). Small Paleozoic detrital zircon populations in SFB may indicate some sediment was sourced from the Maya block (Yucatan) during rifting.

The lack of diverse populations in the South Florida Basin could provide direct insight into the reservoir potential of the basal clastic section in the South Florida Basin. The absence of other sources could indicate that large-scale, regionally integrated drainage systems were not present in southern Florida during the deposition of the basal clastics. Basal clastic sections appear to be relatively thin (100-300 feet) in southern Florida, although distribution is poorly constrained. The thickness and extent of clastics into offshore South Florida is not publicly available, but provenance results suggesting localized, small-scale drainages in onshore southern Florida indicates limited potential for the development of thick sandstone units. Triassic-late Jurassic rift deposits in northern Cuba contain noticeably different provenance signature than the SFB. Previous U-Pb Detrital Zircon Dating of 19 grains from the San Cayetano Formation by Rojas-Agramonte et al. (2008) contained a mix of signatures, including Paleoproterozoic, 
Mesoproterozoic, Neoproterozoic, Ordovician, and Devonian sources. Only one grain consistent with a Pan-African source was identified (561 Ma). The authors interpreted a source from northern South America based on the dominant Mesoproterozoic signature, as well as a possible sediment sources from southern Laurentia or Yucatan. Previous muscovite ${ }^{40} \mathrm{Ar} /{ }^{39} \mathrm{Ar}$ dating from the San Cayetano Formation from Hutson et al. (1998) identified a predominant Paleozoic population which they inferred to have been derived from the Taconic and Alleghenian orogenies. The results from both of these studies suggest that the SFB and syn-rift clastics of Cuba were not derived from the same source, with increased sediment influx from Yucatan or South America toward the south of the early EGOM region.

\section{$\underline{\text { South Georgia Rift }}$}

The presence of both Laurentian and Gondwanan zircons in the SGR implies mixing from multiple sources. The presence of Peri-Gondwanan, Taconic, Acadian, and Alleghenian zircons in SGR syn-rift deposits supports sediment transport from the north. Grenville zircons support at least some sediment transport to the east from the Grenvillie Terrane. The presence of Acadian and Taconic zircons is unusual for the southern Appalachians. Sources may include the Cat Square or other Peri-Gondwanan Terranes with mixed ages, as well as the Alleghanian clastic wedge, and Acadian plutons present in select Peri-Gondwanan Terrains. Paleozoic zircons are present in greater abundances in SGR samples than previously reported studies of the postrift Upper Jurassic Norphlet Formation from southern Alabama Lovell (2010), and the Florida panhandle (Lisi, 2013). Paleozoic zircons may have been sourced locally from unroofing of Paleozoic-aged rocks to source sediment deposited in the SGR, followed by later erosion of Grenville-aged rocks to source the Jurassic deposition of the Norphlet Formation. 
The abundance of Gondwanan zircons in samples 1854 and 3113 in the Tallahassee graben/Albany subbasin, and lower abundance of Gondwanan zircons in samples 442, 108, 3137, and 619 in the Valdosta sub-basin to the north implies that the Suwannee suture is located somewhere between these two rift basins. Samples 442, 619, 108, and 3137 likely lie along the northern ends of the sub-basin, whereas samples 3113 and 1854 likely lie south of the suture. The presence of Gondwana-aged zircon in the Valdosta sub-basin may indicate that the suture is closer to the northern sub-basin, in the very southern extent of Georgia. The detrital zircon results from this study supports the suture location proposed in Mueller et al., (2014) and Mueller et al. (2014; Fig. 72). Norphlet samples P1004 and P1096 from the Florida panhandle from Lovell (2010), and Lisi (2013) contain a mix of Laurentian and Suwannee derived zircons, implying they may also be located proximal to the suture. 


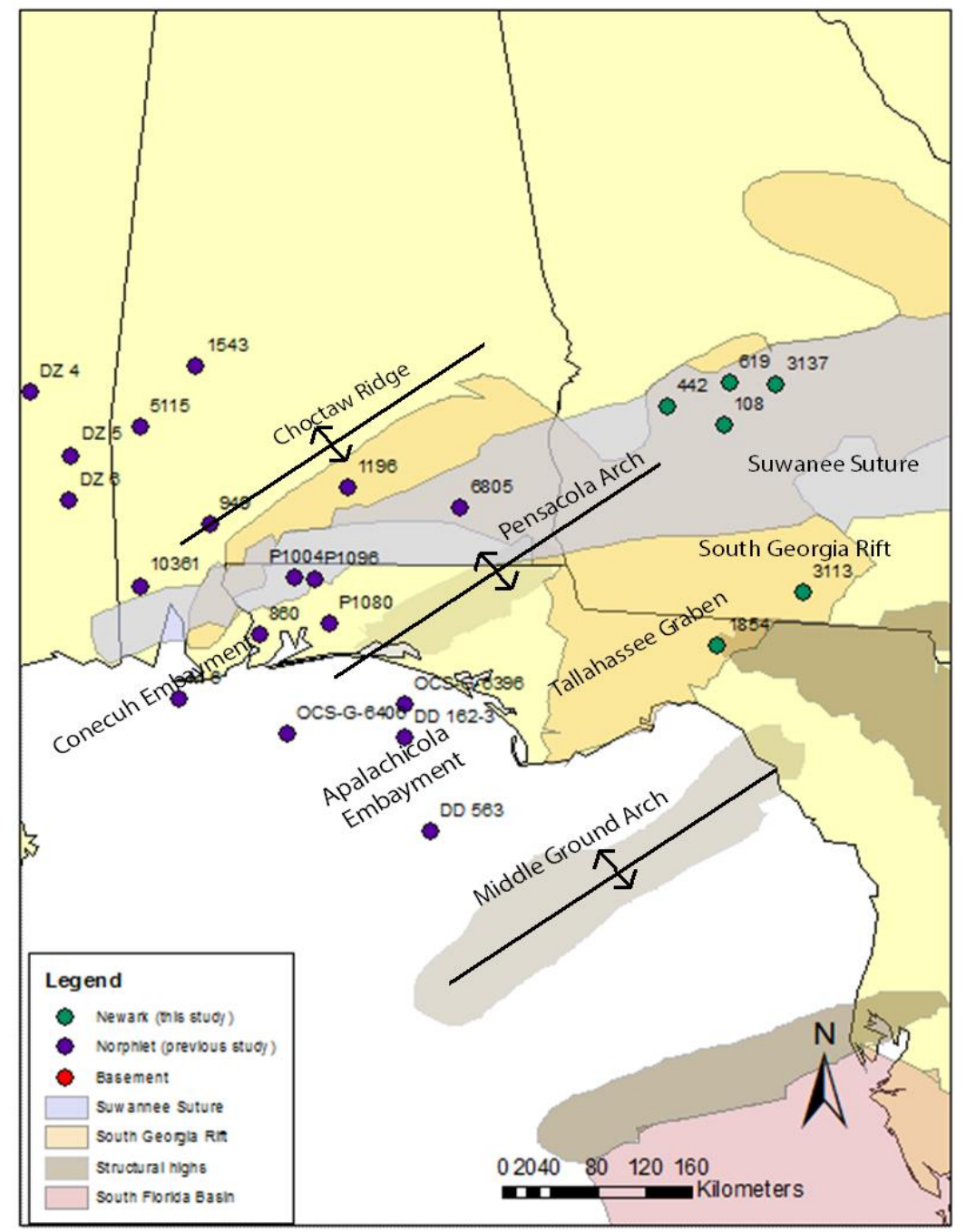

Figure 72. Proposed location of the Suwanne suture, based on work from this study, Lovell, (2010), Lisi (2013), Jesse and Weislogel, (2014) and Heatherington and Mueller, (2014).

\section{$\underline{\text { Triassic/Jurassic Paleo-Transport Systems of Laurentia }}$}

A Paleo-Transport model for drainage systems in Laurentia during the Triassic/Jurassic is displayed in Figure 73. The Appalachians served as a dominant source of sediment throughout North America during the early Mesozoic, and rivers transported sediment as far as the Jurassic dune systems of the west (Navajo, Coconino, etc), where they were then reworked by wind (Rahl et al., 2003; Dickinson and Gehrels, 2009). The Jurassic erg of the western US likely serves as the end of sediment transport from the Appalachians westward. Later, rivers began to drain into 
the opening Gulf of Mexico in the Cretaceous (Blum and Pecha, 2014). Even before the Cretaceous, Appalachian and Peri-Gondwanan rocks also supplied sediment into the EGOM, providing sediment for the Norphlet and other Mesozoic clastics. The Suwannee Terrane served as a major source of sediment for Wood River Formation and also contributed sediment to the younger Norphlet Formation in the northern EGOM, although Suwannee influence decreases towards southern Alabama. Yucatan and South America do not appear to have been major sources of sediment in the northern EGOM during rifting, possibly contributing only minor amounts of detritus into the SFB, and may have at least partially supplied sediment to early Norphlet Formation deposits (Gomes et al., 2016). In contrast, Yucatan likely sourced the San Cayetano Formation in the southern EGOM. 


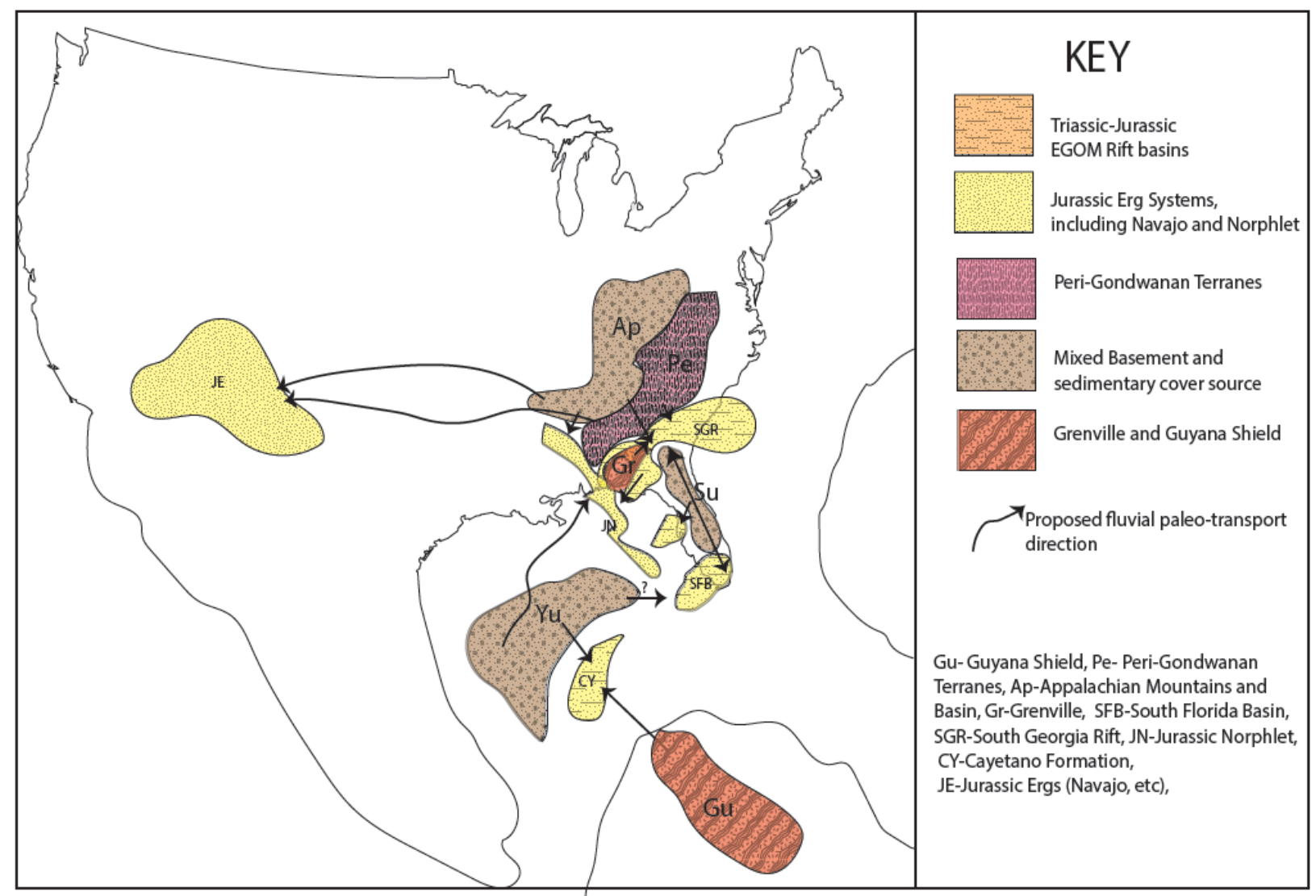

Figure 73. Proposed fluvial paleo-transport model for Laurentia during the Triassic Jurassic. The Appalachian shed sediment that was transported to the Gulf of Mexico, and to the Jurassic ergs systems of the western US. Appalachian sediment was also transported to the Inner Cretaceous Seaway, and southern Canada. Peri-Gondwanan terranes were also a major source of sediment into the western EGOM and the jurassic erg systems. Sediment from Yucatan sourced the San Cayetano Formation, and possibly the South Florida Basin. Modified from Weislogel et al. (2015). Navajo zircon work from Dickinson and Gehrels, (2009), San Cayetano from Rojas-Agramonte et al. (2008).

\section{$\underline{\text { Stratigraphic Correlation }}$}

A proposed stratigraphic correlation for the South Florida Basin, South Georgia Rift, and Eastern Gulf of Mexico is displayed in Figure 74. The minimum age of the Newark Supergroup in the SGR is unknown, but is likely younger than $230 \mathrm{Ma}$, similar to other Newark Rift Basins (Schlische et al., 2003). The minimum age is constrained by post rift diabase and basalt flows related to CAMP volcanism (200 Ma; Schlische et al., 2003). The Newark Supergroup is overlain by interpreted Cretaceous Cotton Valley and other Cretaceous/Cenozoic post-rift strata 
(Chowns and Williams, 1983). A post-rift unconformity separates the Triassic/Jurassic Newark from the Cotton Valley.

The Eagle Mills in the Eastern Gulf of Mexico was likely deposited concurrently with the Newark Supergroup in the South Georgia Rift. An Upper Jurassic, Carnian ( 230 Ma) age is suggested by polynomorphs (Moy and Traverse, 1986). Jurassic/Lower Cretaceous strata overlying the Eagle Mills includes the Werner Anhydrite, Louann Salt, Norphlet, Smackover, Buckner Anhydrite, Cotton Valley. Hosston, and Silgo (Goldhammer, 2001; Mancini et al., 1985; Hammes et al., 2011; Cohen et al., 2013). Sea floor spreading began 160 Ma, following deposition of the Louann Salt (Hudec et al., 2013). Salt deposits are present in northern Yucatan and southern Laurentia, which were initially separated following sea floor spreading. The Norphlet eolian erg was deposited following Louann salt deposition. Based on the age, no correlatable strata are present in the South Georgia Rift. The South Georgia Rift may have partially sourced the Norphlet in the EGOM. Norphlet deposition is followed by anhydrite and carbonate Smackover deposition, which is also not correlated with SGR strata. The Upper Jurassic/Cretaceous Haynesville, Cotton Valley, Hosston, and Silgo likely correlate to Coastal Plain deposits in the SGR ( 125-156 Ma).

The deepest wells in the South Florida Basin have penetrated extrusive rhyolite and basalt, dated 175-199 Ma (Applegate et al., 1981; Heatherington and Mueller, 2003). Very few young zircons were dated from this study, but a Maximum Depositional Age of 195-240 Ma is suggested. The Wood River Formation grades into an upper carbonate interval, with an unknown age. The upper age of the Wood River Formation is constrained by the overlying Bone Island Formation ( 138 Ma; Applegate et al., 1981). The age of the Wood River correlates chronostratigraphically with the Norphlet-Cotton Valley sequence. Salt deposits are also absent 
from the SFB, making correlation difficult. The lower clastic interval was likely deposited concurrently with the Norphlet, while the upper carbonate is correlated with the Smackover. The Lower Cretaceous Pumpkin Bay, Glades Group, and Ocean Reef Group correlate in age to the Cotton Valley, Hosston, Silgo, and other coastal plain deposits in the EGOM and SGR.

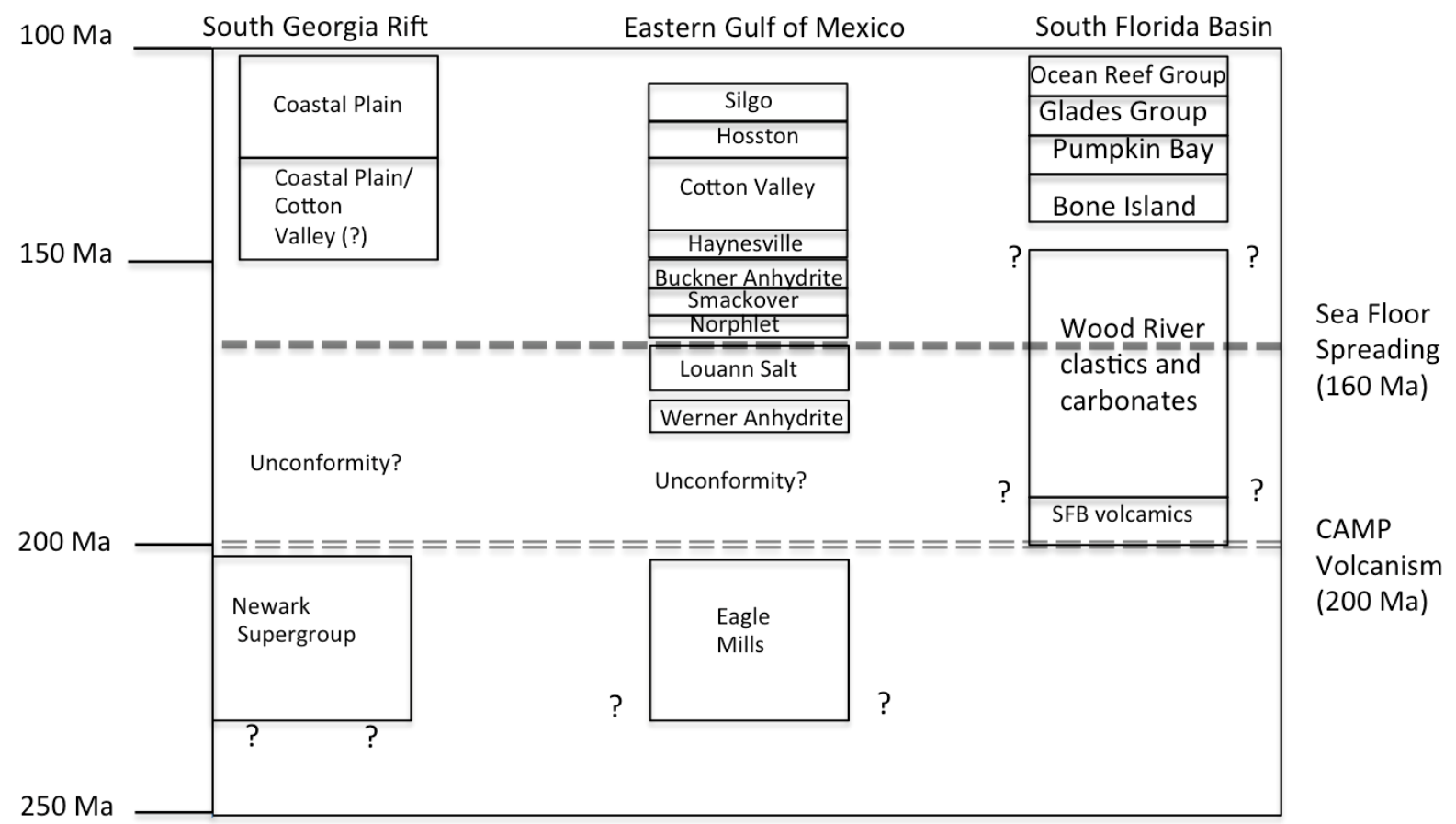

Figure 74. Propsed chronostratigraphic correlation between the SGR, EGOM, and SFB. Correlation is based on formation ages and detrital zircon fresults. SGR ages from Schlische et al. (2003); Chowns and Williams, (1983). EGOM ages from Moy and Traverse, (1986); Goldhammer, (2001); Mancini et al. (1985); Hammes et al. (2001); Cohen et al. (2013). SFB ages from Pollastro et al. (2001); Heatherington and Mueller, (2003); Cohen et al. (2013). Sea floor spreading from Hudec et al. (2013). CAMP ages from Hames et al. (2000). 


\section{Chapter 6: Conclusion}

1. U-Pb of 552 grains from Triassic/Jurassic South Florida Basin clastics identified a dominant Peri-Gondwanan/Gondwanan Population (525-690 Ma) and TransAmazonian/Eburnean population (1900-2200 Ma). The U-Pb age distribution from these samples is consistent with signatures identified in Paleozoic volcanic and metamorphic rocks of Suwannee, indicating the Wood River and other proximal clastics were sourced from local terranes. The uplifted Peninsular Arch likely served as a major sediment source in the Mesozoic before carbonate buildup began. Minor age distributions (12\%) from Laurentia sources may indicate sourcing from Yucatan, or long distance transport by fluvial channels across Florida.

2. A Maximum depositional age of 195-227 Ma is proposed for the Wood River Formation based on the age of the youngest detrital zircon grains. The age of Mesozoic detrital zircons is consistent with a South Florida Volcanic source. The Wood River Formation was likely deposited following initial rifting in the EGOM between 174-235 Ma.

3. Thin-section point-counting indicates a cratonic source for basal sediment in the SFB. The basal sandstones are dominated by subrounded, medium-grained quartz grains, which were derived from Ordovician-Devonian clastics of the Suwannee Terrane. Detrital feldspars and perthites were derived from erosion of the Osceola Granite.

4. Metamorphic phylite sampled from ODP site 0537 yielded zircons with a U-Pb age of $550 \mathrm{Ma}$. This is consistent with a Pan-African origin of this crust. This age is similar to Paleozoic granites and metamorphic rocks in the Suwannee Terrane of Florida, as well as rocks in Western Africa, and Yucatan. The uplifted block at site 537 is likely attenuated continental crust that is an extension of the Suwannee Terrane. 
5. Evaluated wireline logs from the SFB indicate the basin deepens to the south, and the depocenter is likely south of Collier county. V-shale increases to the south, supporting the conclusion that coarse-grained sediment was derived from and deposited proximal to the Peninsular Arch.

6. U-Pb analysis of 567 grains from the Newark Formation within the South Georgia Rift have identified several potential source terranes. These include Appalachian Basin (mixed), Alleghenian (265-330 Ma), Acadian (350-420 Ma), Taconic (430-490 Ma), Gondwanan/Gondwanan Population (525-690 Ma), Mt Rogers (700-760 Ma), Grenville (900-1300 Ma), Granite Rhyolite (1300-1500 Ma), Trans-Amazonian/Eburnean (1900$2200 \mathrm{Ma}$ ), and Yavapai Mazatzal (1600-1800 Ma). The South Georgia Rift was sourced primarily from a mix of Laurentian Appalachian, Peri-Gondwanan, and Suwannee sources. This interpretation is supported by thin section petrography, which identified high feldspar and mica content relative to the SFB.

7. Age distributions in the SGR were used to better constrain the location of the Suwannee suture. Samples 619, 108, 3137, and 442 are all dominated by ages consistent with Laurentian sources, and were deposited on the Laurentia side of the suture, while samples 1854 and 3113 contain relatively large Peri-Gondwanan/Gondwanan populations relative to the other SGR samples, and were likely deposited at or near the Suwannee suture.

8. U-Pb age signatures from the SFB and SGR were compared to previous Norphlet Formation provenance studies by Lovell (2010) and Lisi (2013) using the K-S test to identify if the Norphlet may have shared potential sources with syn-rift deposits in the SGR or SFB. Eight major regions with distinct age distributions were identified. Laurentian sources dominate sediment supplied to southern Alabama, Gondwanan 
sources dominate sediment supplied to southern Florida, and a mixed source of Laurentia and Suwannee crust supplied sediment to the region between southern Florida and southern Alabama.

9. Age distributions of Triassic Newark sediment from the South Georgia Rift are statistically similar to offshore Jurassic Norphlet samples from the Apalachicola Embayment. The two regions likely shared similar sources, and the SGR/Tallahassee Graben may have served as a potential sediment source into the EGOM. 


\section{References}

Akintunde, O.M., Knapp, C., and Knapp, J., 2013, Petrophysical Characterization of the South Georgia Rift Basin for Supercritical $\mathrm{CO}_{2}$ Storage: A Preliminary Assessment: Earth Environmental Sciences, v. 70, p. 2971-2985.

Aleinkoff, J.N., Zartman, R.E., Walter, M., Rankin, D.W., Lyttle, P.T., and Burton, W.C., 1995, $\mathrm{U}-\mathrm{Pb}$ Ages of Metarhyolites of the Catoctin and Mount Rogers Formations, Central and Southern Appalachians: Evidence for Two phases of Iatetan Rifting: American Journal of Science, v. 295, p. 428-454.

Applegate, A.V., Winston, G.O., and Palacas, J.P., 1981, Subdivision and Regional Stratigraphy of the Pre-Punta Gorda Rocks (Lowermost Cretaceous-Jurassic?) in South Florida: Gulf Coast Association of Geological Societies, v. 31, p. 447-453.

Applin, P.L., and Applin, E.R., 1965, The Comanche Series and Associated Rocks in the Subsurface in Central and South Florida: U.S Geol. Survey Prof. Paper 447, 84 p.

Arthur, J. D., 1988, Petrogenesis of Early Mesozoic Tholeiite in the Florida Basement Geology: Florida Geological Survey, v. 97, p. 39.

Austin, J.A., Stoffa, P.L., Phillips, J.D., Oh, J., Sawyer, D.S., Purdy, G.M., Reiter, E., and Makris, J., 1990, Crustal Structure of the Southeast Georgia Embayment-Carolina Trough: Preliminary Results of a Composite Seismic Image of a Continental Structure (?) and a Volcanic Passive Margin: Geology, v. 18, p. 1023-1027.

Becker, T.P., Thomas, W.A., Samson, S.D., and Gehrels, G.E., 2005, Detrital Zircon Evidence of Laurentian Crustal Dominance in the Lower Pennsylvanian Deposits of the Alleghanian Clastic Wedge in Eastern North America: Sedimentary Geology, v. 182, p. 59-86.

Blakey, R., 2016, Coloaro Plateau Stratigraphy and Geology and Global and Regional Paleogeography, World Wide Web, https://www2.nau.edu/rcb7/ (accessed February, 2016).

Blum, M., and Pecha, M., 2014, Mid-Cretaceous to Paleocene North American Drainage Reorganization from Detrital Zircons: Geology, v. 42, p. 607-610.

Boote, S.K., and Knapp, J.H., 2016, Offshore Extent of Gondwanan Paleozoic Strata in the Southeastern United States: The Suwannee Suture Zone Revisited: Gondwana Research, v. 40, p. 199-210.

Boschman, L.M., Van Hinsbergen D.J.J., Torsvik, T.T., Spakman, W., and Pindell, J.L., 2014, Kinematic Reconstruction of the Caribbean Region Since the Early Jurassic: Earth Sciences Review, v. 138, p. 102-136. 
Bovay, A.C., Snedden, J, W., Steel, R, J., and Ganey-Curry P.E., 2014, New Models of Valanginian Source-to-Sink Pathways in the Eastern Gulf of Mexico: Gulf Coast Association of Geological Societies: v. 64, p. 507-509.

Bowman, S.A., 2012, Exploration Targets of Offshore Western Florida: Gulf Coast Association of Geological Societies Transactions, v. 62, p. 13-26.

Bream, B.R., Hatcher, R.D., Miller, C.F., and Fullagar, P.D., 2004, Detrital Zircon Ages and Nd Isotopic Data from the Southern Appalachian Crystalline Core, GA-SC-NC-TN: New Provenance Constraints for Part of the Laurentian Margin: In Tollo RP, Corriveau L., McLelland, J., and Bartholomew, M.J., eds., Proterozoic Tectonic Evolution of the Grenville Orogen in North America: Geological Society of America Memoir, v. 197, p. 459-475.

Buffler, R.T., Schlager, W., Bowdler, J.L., Cotillon, P.H., Halley, R.B., Kinoshita, H., Magoon, L.B., McNulty, C.L., Patton, J.W., Silva, I.P., Suarez, O.A., Testarmata, M.M., Tyson, R.V., and Watkins, D.K., 1984, Site 0537, Initial Reports Reports of the Deep Sea Drilling Project Volume 77, U.S Government Printing Office, p. 255-278.

Buffler, R.T., Schlager, W., Bowdler, J.L., Cotillon, P.H., Halley, R.B., Kinoshita, H., Magoon, L.B., McNulty, C.L., Patton, J.W., Silva, I.P., Suarez, O.A., Testarmata, M.M., Tyson, R.V., and Watkins, D.K., 1984, Site 0538, Initial Reports Reports of the Deep Sea Drilling Project Volume 77, U.S Goverment Printing Office, p. 279-336.

Butler, J.R., and Fullagar, P.D., 1978, Plate-Tectonic Model for the Southern Appalachians in the Carolinas: Geological Society of America Abstract Programs, v. 20, p. 164.

Burton, W.C., Southworth, S., 2010, A Model for Iapetan Rifting of Laurentia Based on Neoproterozoic Dikes and Related Rocks, in Tollo, R.P., Bartholomew, M.J., Hibbard, J.P., and Karabinos, P.M., eds., From Rodinia to Pangea: The Lithospheric Record of the Appalachian Region: Geological Society of America Memoir, v. 206, p. 455-476.

Bureau of Ocean Energy Management, Areas Under Moratoria, accessed April, 2016, https://www.boem.gov/Areas-Under-Moratoria

Carrigan, C.W., Miller, C.F., Fullagar, P.D., Hatcher, R.D., Jr., Bream, B.R., and Coath, C.D., 2003, Ion Microprobe Age and Geochemistry of Southern Appalachian Basement, with Implications for Proterozoic and Paleozoic Reconstructions: Precambrian Research, v. 120, p. 136.

Carter, B.T., Hibbard, J.P., Tubrett, M., and Sylvester, P., 2006, Detrital Zircon Geochronology of the Smith River Allocthon and Lynchburg Group, Southern Appalachians: Implications for Neoproterozoic-Early Cambrian Paleogeography: Precambrian Research, v. 147, p. 279-304. 
Chowns, T.M., and Willians, C. T. 1983, Pre-Cretaceous Rocks Beneath the Georgia Coastal Plain-Regional Implications, in Gohn, G.G, eds., Studies Related to the Charleston, South Carolina Earthquake of 1886-Tectonics and Seismicity: USGS Professional Paper 1313 L, p. 42.

Claypool, G.E., and Mancini, E.A., 1989, Geochemical Relationships of Petroleum in Mesozoic Reservoirs to Carbonate Source Rocks of Jurassic Smackover Formation, Southwestern Alabama: The American Association of Petroleum Geologists Bulletin, v. 73, p. 904-924.

Clendenin, C.W., 2013, Insights Into the Mode of the South Georgia Rift Extension in Eastern Georgia, USA: Tectonophysics, v. 26, p. 613-621.

Cohen, K.M., Finney, S.L., Gibbard, P.L., 2013, International Chronostratigraphic Chart: International Commision on Stratigraphy, v 2013/01.

Coleman, J.L., Milici, R.C., and Post, P.J., Assessment of the Oil and Natural Gas Potential of the East Coast Mesozoic Synrift Basins, Onshore and State Waters of the United States: $34^{\text {th }}$ Annual GCSSPEM Conference, Houston TX, December 13-16 ${ }^{\text {th }}, 2015$.

Coler, D.G., Wortman, G.L., Samson, S.D., Hibbard, J.P., and Stern, R., 2000, U-Pb Geochronologic, $\mathrm{Nd}$ isotopic, and Geochemical Evidence for the Correlation of the Chopawamsic and Milton Terranes, Piedmont Zone, Southern Appalachian Orogen: Journal of Geology, v. 108, p. 363-380.

Cordani, U.G., Sato, K., Teixeira, W., Tassinari, C.G., and Basei, M.A.S., 2000, Crustal Evolution of the South American Platform, in Cordani, U.G., Milani, E.J., Thomaz Filho, A.Campos, D.A., Tectonic Evolution of South America: $31^{\text {st }}$ at IGC, Special Publication, 19-40.

Cordani, U.G., Teixeira, W., D’Agrella-Filho, M.S., and Trinadade, R.I.F., 2009, The position of the Amazonian Craton in Supercontinents, Gondwana Research: v. 15, p. 396-407.

Culver, S.J., and Hunt, D., 1993, Lithostratigraphy of the Precambrian-Cambrian Boundary Sequence in the Southwestern Taoudeni Basin, West Africa: Journal of African Earth Sciences (and the Middle East), v. 13, p. 407-413.

Dallmeyer, R.D., 1989, A Tectonic Linkage Between the Rokelide Orogen (Sierra Leone) and the St. Lucie Metamorphic Complex in the Florida Subsurface: Journal of Geology vol. 89, p. 183-195.

Dallmeyer, R.D., 1989, Contrasting Accreted Terranes in the Southern Appalachian Orogen and Atlantic-Gulf Coastal Plains and Their Coastal Plains and Their Correlation with West African Sequences, in Dallmeyer, R.D., ed., Terranes in the Circum-Atlantic Paleozoic Orogens: Geological Society of America Special Paper 230, p. 247-267.

Dallmeyer, R.D., 1983, ${ }^{40} \mathrm{Ar} /{ }^{39} \mathrm{Ar}$ Ages From a Pre-Mesozoic Crystalline Basement Penetrated At Holes 537 and 538A of the Deep Sea Drilling Project Leg 77, Southeastern Gulf of Mexico: 
Tectonic Implications: Initial Reports Reports of the Deep Sea Drilling Project Volume. 77, U.S Govt Printing Office, 1984 p. 497-504.

Dallmeyer, R.D., and Lecorche, J.P., 1987, ${ }^{40} \mathrm{Ar} /{ }^{39} \mathrm{Ar}$ Mineral Age Record of Polyphase Tectonothermal Evolution in the Southern Mauritanide Orogen, Southeastern Sengal: Geological Society of America Bulletin, v. 98, p. 602-611.

Delor, C., Lafon, J.M., Millesi, J.P., and Fanning M., 2002, First Evidence of 560-575 Ma Granulites and Syn-Tectonic Magmatism in the Rokelides Belt: Geology, Geochronology and Geodynamic Implications, 19 ${ }^{\text {th }}$ CAG, El Jadida, Morocco, 19-22 March, 2002.

Dickinson, W.R., and Gehrels, G.E., 2009, U-Pb Ages of Detrital Zircons in Jurassic Eolian and Associated Sandstones of the Colorado Plateau: Evidence for Transcontinental Dispersal and Intraregional Recycling of Sediment: Geological Society of America Bulletin, v. 121, p. 408433.

Dickinson, W.R., and Gehrels, G.E., 2009, Use of U-Pb Ages of Detrital Zircons to Infer Maximum Depositional Ages of Strata: A Test Against a Colorado Plateau Mesozoic Database: Earth and Planetary Science Letters, v. 288, p. 115-125.

Dickinson, W.R., and Lawton, T.F., 2001, Carboniferous to Cretaceous Assembly and Fragmentation of Mexico: GSA Bulletin, v. 113, p. 1142-1160.

Daniels, D.L., Zietz, I., and Popenoe, P., 1983, Distribution of Subsurface Lower Mesozoic Rocks in the Southeastern United States as Interpreted from Regional Aeromagnetic and Gravity Maps in Gohn, G.S., eds., Studies Related to the Charleston, South Carolina, Earthquakes of 1886-Tectonics and Seismicity: U.S Geological Survey Professional Paper 1313, p. K1-K24.

Dickinson, W.R., and Suczek, C.A, 1979, Plate Tectonics and Sandstone Compositions: AAPG Bulletin, v. 12, p. 2165-2182.

Dobson, L.M., and Buffler, R.T., 1997, Seismic Stratigraphy and Geologic History of Jurassic Rocks, Northeastern Gulf of Mexico: AAPG Bulletin, v. 81, p. 100-120.

Eisenstadt, G., and Withjack, M.O., 1995, Estimating Inversion: Results from Clay Models, in Buchannan, JG., and Buchanan, P.G., eds., 1995, Basin Inversion: Geological Society of London Special Publication 88, p. 119-136.

Ettensohn, F.R., 2004, Modeling the Nature and Development of Major Paleozoic Clastic Wedges in the Appalachian Basin, USA: Journal of Geodynamics, v. 37, p. 657-681.

Ennih, N., and Liègeois, J., 2008, The Boundaries of the West African Craton, with Special Reference to the Basement of the Moroccan Metacratonic Anti-Atlas Belt: The Geological Society, v. 297, p. 1-17.

Eswai, E.K., 2004, Evidence from the Farmington Pluton for Early Devonian Subduction Related Magmatism in the Carolina Zone of Central North Carolina: Journal of Geodynamics, v. 37, p. 531-548. 
Fetter, A.H., Goldberg, S.A., 1995, Age and Geochemical Characteristics of Bimodal Magmatism in the Neoproterozoic Grandfather Mountain Rift Basin: Journal of Geology, v. 103, p. 311-323.

Fisher, C.M., Loewy, S.L., Miller, C.F., Berquist, P., Vans Schmus, W.R., Hatcher, R.D., Jr., Wooden, J.L., and Fullagar, P.D., 2010, Whole-rock Pb and Sm-Nd isotopic Constraints on the Growth of the Southeastern Laurentia During Grenvillian Orogenesis: Geological Society of America Bulletin, v. 122, p. 1646-1659.

Folk, 1980, Petrology of Sedimentary Rocks: Hemphill Publishing Company,

Fowell, S.J., Cornet, B., and Olsen, P.E., 1994, Geologically Rapid Late Triassic Extinctions: Palynological Evidence From the Newark Supergroup: v. 288, p. 197-206.

Fullagar, P.D., Butler, J.R., 1979, 325 to 265 M.Y Old Granitic Plutons in the Piedmont of the Southeastern Appalachians: American Journal of Science, v. 279, p. 161-185.

Galloway, W.E., Whiteaker, T.L., and Ganey-Currey, P., 2011, History of North American Drainage Basin Evolution, Sediment Yield, and Accumulation in the Gulf of Mexico basin: Geosphere, v. 7, p. 938-973.

Gehrels, G.E., 2014, Detrital Zircon U-Pb Geochronology Applied to Tectonics: Annual Review of Earth and Planetary Sciences: v. 42, p. 127-149.

Gehrels, G.E., Calencia, V.A., and Ruiz, J., 2008, Enhanced Precision, Accuracy, Efficiency, and Spatial Resolution of U-Pb Ages by Laser Ablation-Multicollector-Inductively Coupled PlasmaMass Spectrometry: Geochemistry Geophysics Geosystems, v. 9, p. 1-13.

Gehrels, G.E., 2012, Detrital Zircon U-Pb Geochronology: Current Methods and New Opportunities, in Busby, C., and Azor, A., eds., Tectonics of Sedimentary Basins: Recent Advances, First Edition.

Godo, T., 2006, Norphlet Aeolian Dunes in the Deep Water Gulf of Mexico: Houston Geological Survey Bulletin, v. 49, p. 11.

Goldhammer, R.K., Johnson, C.A., 2001, Middle Jurassic-Upper Cretaceous Paleogeographic Evolution and Sequence Stratigraphic Framework of the Northwest Gulf of Mexico Rim, in Bartolini, C., Cantu-Chapa, A., Buffer, R.T., eds., The Western Gulf of Mexico Basin: Tectonics, Sedimentary Basins and Petroleum Systems: AAPG Memoir, v. 75, p. 45-81.

Goldberg, S.A., Butler, J.R., and Fullagar, P.D., 1986, The Bakersville Dike Swarm, Geochronology and Petrogenesis of Late Proterozoic Basaltic Magmatism in the Southern Appalachian Blue Ridge: American Journal of Science, v. 286, p. 403-430.

Gomes, S., Weislogel, A., Robinson, D., and Barbeau, D., Temporal Variation in U-Pb Geochronology of the Norphlet Formation in the Eastern Gulf of Mexico, Mobile Bay: Connection Between Sediment Provenance and Reservoir Quality: AAPG Annual Convention and Exhibition, Calgary, AB, June 19-22, 2016. 
Gregg, A.C., 2014, Tectonic Evolution of the West Florida Basin, Eastern Gulf of Mexico: University of Alabama, Master's thesis. p. 61

Gutiérrez, M.A., Ewing, R., 2015, Study of the Spatial Variations in Eolian Stratigraphic Architecture in the Norphlet Formation, Southwestern Alabama: Texas A and M, Masters thesis, p. 1-67.

Haq, B.U., Hardenbol, J., and Vail, P.R., 1987, Chronology of Fluctuating Sea Levels Since the Triassic: Science, v. 235, p. 1156-1167.

Hames, W.E., Renne, P.R., and Ruppel, C., 2000, New Evidence for Geologically-Instantaneous Emplacement of Earliest Jurassic Central Atlantic Magmatic Province Basalts on the North American Margin. Geology, v. 28, p. 859-862.

Hammes, U., Hamlin, H, S., and Ewing, T.E., 2011, Geologic Analysis of the Upper Jurassic Haynesville Shale in east Texas and West Louisiana; AAPG Bulletin, v. 95, p. 1643-1666.

Harrison, T.M., Duncan, I., and McDougall, I., 1985, Diffusion of ${ }^{40} \mathrm{Ar}$ in Biotite: Temperature, Pressure, and Compositional Effects: Geochemical et Cosmochimica Acta, v. 49, p. 2461-2468.

Hatcher, R.D., 1987, Tectonics of the Southern and Central Appalachian Internides: Annual Review of Earth and Planetary Science, v. 15, p. 337-362.

Hatcher, R.D.,1989, Tectonic Synthesis of the U.S Appalachians, in Hatcher, R.D., Jr., Thomas, W.A., and Viele, G.W., eds., The Appalachian-Ouachita Orogen in the United States: Geological Society of America, Geology of North America, v. F-2, p. 511-535.

Hatcher, R.D., Jr., 2010, The Appalachian Orogen: A Brief Overview, in Tollo, R.P., Bartholomew, M.J., Hibbard, J.P., Karabinos, P.M. eds., From Rodinia to Pangea: The Lithotectonic Record of the Appalachian Region: Geological Society of America Memoir, v. 206, p. 1-19.

Hatcher, R.D., Bream, B.R., and Merschat, A.J., 2007, Tectonic Map of the Southern and Central Appalachians: A Tale of Three Orogens and a Complete Wilson Cycle, in Hatcher, R.D., Carlson, M.P, McBride, J.H., and Martínez Catalán, J.R., eds., 4-D Framework of Continental Crust: Geological Society of America Memoir 200, p. 595-632.

Hatcher, R.D., Brendan, B.R., Mershcat, A.J., Russell, M, W., and Miller, C.F., 2005, Evidence for the (Neo-) Acadian Orogeny in the Southern Appalachians: Geological Society of America Southeastern Section, v. 37, p. 5.

Hatcher, R.D., JR., Bream, B.R., Miller, C.L., Eckert, J.O., Jr., Fullagar, P.D., and Carrigan, C.W., 2004, Paleozoic Structure of Internal Basement Massifs, Southern Appalachian Blue Ridge, Incorporating New Geochronologic Nd and Sr Isotopic and Geochemical data, in Tollo, R.P., Corriveau, L., McLelland, J., and Bartholomew, M.J., eds., Proterozoic Tectonic Evolution 
of the Grenville Orogen in North America: Geological Society of America Memoir v. 197, p. 525-547.

Heatherington, A.L., and Mueller, P.A., 1991, Geochemical Evidence for Triassic Rifting in Southwestern Florida: Tectonophysics, v. 199, p. 291-302.

Heatherington, A.L, and Mueller, P.A, 1997, Geochemistry and Origin of Floridan Crustal Basement Terranes, in Randazzo, A., Jones, D. eds., Geology of Florida: University Press of Florida, Gainesville, p. 27-37.

Heatherington, A.L, and Mueller, P.A, 1999, Lithospheric sources of North Florida, USA Tholeiites and Implications for the Origin of the Suwannee Terrane: Lithosphere, v. 46, p. 215 333.

Heatherington, A.L., and Mueller, P.A., 2003, Mesozoic Igneous Activity in the Suwannee Terrane, Southeastern USA: Petrogenesis and Gondwanan Affinities: Gondwana Research, v. 6, p. 296-311.

Heatherington, A.L., Mueller, P.A., and Nutman, A.P., 1999, A Jurassic Granite from Southern Georgia, U.S.A.: Silicic, Extension-Related Magmatism Along the Southern Coastal Plain, v. 107, p. 375-384.

Heatherington, A.L., Mueller, P.A., Spencer, J., and Isachsen, C.E., 1993, Terrane Accretion in Southeastern North America: Constraints of Geochronologic Data from Paleozoic and Proterozoic Grainitoids: Geological Society of America Abstracts with Programs, v. 25, p. 322.

Heffner, D.M., 2013, Tectonics of the South Georgia Rift, University of South Carolina, Ph.D Dissertation, p. 177.

Hibbard, J.P., Stoddard, E.F., Secor, D., and Dennis, A., 2002, The Carolina Zone: Overview of Neoproterozoic to Early Paleozoic Peri-Gondwanan Terranes Along the Eastern Flank of the Southern Appalachians: Earth Science Reviews, v. 57, p. 299-339.

Hibbard, J.P., Van Staal, C.R., Rankin, D.W., 2010, Comparative Analysis of the Geological Evolution of the Northern and Southern Appalachian Orogen: Late Ordovician-Permian, in Tollo, R.P., Bartholomew, M.J., Hibbard, J.P., and Karabinos, P.M., eds., From Rodinia to Pangea: The Lithospheric Record of the Appalachian Region: Geological Society of America, Memoir v. 206, p. 51-69.

Hibbard, J.P., 2013, One Arc, Two Arcs, Old Arc, New Arc: The Carolina Terrane in the Southern Appalachians of North Carolina, Geological Society of America Abstracts with Programs: v. 45, no. 7, p. 292.

Hine, A.C., Suthard, B.C., Locker, S.D., Cunningham, K.J., Duncan, D.S., Evans, M.W., and Morton, R.A., 2009, Karst Sub-basins and Their Relation to the Transport of Tertiary 
Siliciclastic Sediments on the Florida Platform, in Stewart, P.K., Swart, G.P. Eberli G.P., and McKenzie, Prespectives in Carbonate Geology: A Tribute to the Career of Robert Nathan Ginsburg, eds. 179-97.

Hodych, J.P., and Dunning, G.R., 1992, Did the Manicouagan Impact Trigger End-Of-Triassic Mass Extinction: Geology, v. 20, p. 51-54,

Hooper, R.J., and Hatcher, R.D., 1989, The Origin of Ultramafic Rocks from the Berner Mafic Complex, Central Georgia, in Mittwede, S.K., Stoddard, E.F., Ultramafic Rocks of the Appalachian Piedmont., eds., Geological Survey of America Special Papers, v. 231, p. 87-92.

Horton, J.W., Drake, A.A., and Rankin, D.W., 1989, Tectonostratigraphic Terranes and Their Paleozoic Boundaries in the Central and Southern Appalachians, in Dallmeyer, R.D., eds., Terranes in the Circum-Atlantic Paleozoic Orogens: Geological Society of America Special Paper., v. 230, p. 213-245.

Hoskin, P.W., and Schaltegger, U., 2003, The Composition of Zircon and Igneous and Metamorphic Petrogenesis: Reviews in Mineralogy and Geochemistry, v. 53, p. 27-62.

Hudec, M.R., Norton, I.O., and Jackson, M.P.A., Peel, F.J., 2013, Jurassic Evolution of the Gulf of Mexico Salt Basin: AAPG Bulletin, v. 97, p. 1683-1710.

Hunt, B.W., 2012, Regional Norphlet Facies Correlation, Analysis and Implications for Paleostructure and Provenance, Eastern Gulf of Mexico: Master's thesis, University of Alabama, p. 112.

Hutson, F., Mann, P., and Renne, R., 1998, ${ }^{40} \mathrm{Ar} /{ }^{39} \mathrm{Ar}$ Dating of Single Muscovite Grains in Jurassic Siliciclastic Rocks (San Cayetano Formation): Constraints on Paleo position of Western Cuba: Geology, v. 26, p. 83-86.

Hunter, I., 2014, Origin and Development of the Apalachicola Basin: University of Alabama, Master's Thesis, p. 98.

Jesse, M. L., and Weislogel, A., 2014, Depositional Analysis of the Jurassic Norphlet Formation Within the Mississippi Interior Salt Basin: Impact on Petroleum Potential Offshore: American Association of Petroleum Geologists Annual Meeting, Houston, TX, April 6-9.

Keppie, J.D., and Ortega-Gutiérrez, F., 1999, Middle American Precambrian Basement: A Missing Piece of the Reconstructed 1-Ga Orogen., in Ramos, V.A. and Keppie, J.D. LaurentiaGondwana Connections Before Pangea., eds., Geological Society of America Special Papers, v. 336, p. 199-210.

Keppie J.D, Nance, R.D, Murphy, J.B, and Dostal, J., 2003, Tethyan, Mediterranean, and Pacific Analogues for the Neoproterozoic-Paleozoic Birth and Development of Gondwanan Terranes and Their Transfer to Laurentia and Laurussia: Tectonophysics, v. 365, p. 195-219. 
Klitgord, K.D., Hutchinson, D.R., and Schouten, H., 1988, U.S Atlantic Continental Margin; Structural and Tectonic Framework., in Sheridan, R.E., and Grow, J.A., The Geology of North America, v. 1-2, The Atlantic Continental Margin, U.S Geological Society of America, p. 19-56.

Korgh, T.E., Kamo, S.L., Sharpton, V.L., Martin, L.E., and Hildebrand, A.R., 1993, U-Pb Ages of Single Shocked Zircons Linking Distal K/T Ejecta to the Chicxulub Crater: Nature, v. 366, p. 731-734.

Lisi, A,F., 2013, Provenance of the Upper Jurassic Norphlet and Surrounding Formations U-Pb Detrital Zircon Geochronology: West Virginia University, Master's thesis. p. 148.

Lovell, T.R., and Weislogel, A., 2010, Detrital Zircon U-Pb Age Constraints of the Provenance of the Upper Jurassic Norphlet Formation, Eastern Gulf of Mexico: Implications for Paleogeography: Gulf Coast Association of Geological Societies Transactions, v. 60, p. 443-460.

MacRae, M., and Steltenpohl, M.G., 1993, Geology of Part of the Uchee Belt, Eastern Alabama, and its Implications for Alleghanian Development., in Steltenpohl, M.G., and Salpas, P.A., eds., Geology of the Southernmost Exposed Appalachian Piedmont Rocks along the Fall Line: $42^{\text {nd }}$ Annual Geological Society of America Field Trip Guidebook, p. 142-168.

Mancini, E.A., Mink, R.M., and Bearden, B.L., 1984, Paleoenvironments and Hydrocarbon Potential of the Upper Jurassic Norphlet Formation of Southwestern and Offshore Alabama: Gulf Coast Association of Geological Societies Transactions, v. 34, p. 131-135.

Mancini, E.A., Mink, R.M, Bearden, B.L., and Wilkerson, R.P., 1985, Norphlet Formation (Upper Jurassic) of Southwestern and Offshore Alabama: Environments of Deposition and Petroleum Geology: AAPG Bulletin, v. 69, p. 881-898.

Mancini, E, A., Llinás, J.C., Parcell, W.C., Aurell, M., Bádenas, B., Leinfelder, R.R., and Benson, D.J., 2004, Upper Jurassic Thrombolite Reservoir Play, Northeastern Gulf of Mexico: AAPG Bulletin, v. 88, p. 1573-1602

Mancini, E.A., Tew, B.H., and Mink, R.M., 1990, Jurassic Sequence Stratigraphy in the Mississippi Interior Salt Basin of Alabama: Gulf Coast Association of Geological Societies Transactions, v. 40, p. 521-530.

MacRae, G., and Watkins, J.S., 1993, Basin Architecture, Salt Tectonics, and Upper Jurassic Structural Styles, Desoto Canyon Salt Basin, Northeastern Gulf of Mexico: v. 77, p. 1809-1824.

MacRae, G., and Watkins, J.S., 1995, Early Mesozoic Rift Stage Half Graben Formation Beneath the DeSoto Canyon Salt Basin Northeastern Gulf of Mexico: Journal of Geophysical Research, v.100, p. 17795-17812.

Marzoli, A., Renne, P.R., Piccirillo, E.M., Ernesto, M., Bellieni, G., and Min, A.D., 1999, Extensive 200-Million-Year-Old Continental Flood Basalts of the Central Atlantic Magmatic Province: Science, v. 284, p. 616-618. 
Mezger, K., and Krogstad, J., 1997, Interpretation of Discordant U-Pb Zircon Ages: An Evaluation: Journal of Metamorphic Geology, v. 15, p. 127-140.

McBride, J.H., Hatcher, R.D., Stephenson, W.J., and Hooper, R.J, 2005, Integrating Seismic Reflection and Geological Data and Interpretations Across an Internal Basement Massif: The Southern Appalachian Pine Mountain Window, USA: Geological Society of American Bulletin, v. 117 , p. 669-686.

McBride, J.H., Nelson, K.D., and Brown, L.D., 1989, Evidence and Implications of an Extensive Mesozoic Rift Basin and Basalt/Diabase Sequence Beneath the Southeast Coastal Plain: Geological Society of America Bulletin, v. 101, p. 512-520.

McLelland, J.M., Selleck, B.W., and Bickford, M.E., 2010, Review of the Proterozoic Evolution of the Grenville, Province, its Adirondack Outlier, and the Mesoproterozoic Inliers of the Appalachians, in Tollo, R.P., Bartholomew, M.J., Hibbard, J.P., and Karabinos, P.M., eds., From Rodinia to Pangea: The Lithospheric Record of the Appalachian Region: The Geological Society of America Memoir v. 206, p. 21-49.

McSween, H.Y., Speer J.A, and Fullagar P.D., 1991, Plutonic Rocks, in The Geology of the Carolinas., in J.W. Horton, and Zulli, V.A., eds., The Geology of the Carolinas: Carolina Geological Society Fiftieth Anniversary Volume: University of Tennessee, Press, Knoxville, p. 109-126.

Merschat, A.J., Bream, R.D., Miller, C.F., Byars, H.F., Catewood, M.P., and Wooden, J.L., 2010, Detrital Zircon Geochronology and Provenance of Southern Appalachian Blue Ridge and Inner Piedmont Crystalline Terranes., in Tollo, R.P., Bartholomew, M.J., Hibbard, J.P., and Karabinos, P.M., eds., From Rodinia to Pangea: The Lithospheric Record of the Appalachian Region: Geological Society of America Memoir, v. 206, p. 661-699.

Merschat, A.J., and Hatcher, R.D., 2007, The Cat Square Terrane: Possible Siluro-Devonian Remnant Ocean Basin in the Inner Piedmont, Southern Appalachians, USA, in Hatcher, R.D., Jr., Carlson, M.P., McBride, J.H., and Martinez Catalàn, J.R., eds., 4-D Framework of Continental Crust: Geological Society of America Memoir v. 200, p. 553-566.

Mershcat, A.J., Hatcher, R.D., Jr., and Davis, T.L., 2005, The Northern Inner Piedmont, Southern Appalachians, USA: Kinematics of Transpression and SW-directed Mid-Crustal Flow: Journal of Structural Geology, v. 27, p. 1252-1291.

Mitchell-Tapping, H.J., 1994, Jurassic and Triassic Hydrocarbon Exploration of Southern Florida, Transactions of the Gulf Coast Association of Geological Societies, v. 44, p. 507-513.

Milici, R.C., Coleman, J.L., Rowan, E.L., Cook, T.A., Charpentier, R.R., Kirschbaum, M.A., Klett, T.R., Pollastro, R.M., and Schenk, C.J., 2011, Assessment of Undiscovered Oil and Gas Resources of the East Coast Mesozoic Basins of the Piedmont, Blue Ridge Thrust Belt, Atlantic Coastal Plain, and New England Provinces: United States Geological Survey Fact Sheet 20123075 . 
Miller, J.A., 1986, Hydrologic Framework of the Floridan Aquifer System in Florida and in Parts of Georgia, Alabama, and South Carolia: U.S Geological Survey Professional Paper 1403-B, B23-B35.

Miller, B.V., Fetter, A.H., and Stewart, K.G., 2006, Plutonism in Three Orogenic Pulses, Eastern Blue Ridge Province, Southern Appalachians: Geological Society of America Bulletin, v. 118, p. 171-184.

Miller, C.F., Hatcher, R.D., Ayers, J.C., Coath, C.D., and Harrison, T.M., 2000, Age and Zircon Inheritance of Eastern Blue Ridge Plutons, Southwestern North Carolina and Northeastern Georgia, with Implications for Magma History and Evolution of the Southern Appalachians Orogen: American Journal of Science, v. 300, p. 142-172.

Moecher, D.P., Samson, S.D., and Miller, C.F., 2004, Precise Time and Conditions of Peak Taconian Granulite Facies Metamorphism in the Southern Appalachian Orogen, USA with Implications for Zircon Behavior During Crustal Melting Events: The Journal of Geology, v. 112, p. 289-304.

Montgomery, S.L., 2000, Wiggins Arch, Southern Mississippi: New Exploratory Data from 3-D Seismic: AAPG Bulletin, v. 84, p. 299-313.

Moy, C., and Traverse, A., Palynostratigraphy of the Subsurface Eagle Mills Formation (Triassic) From a Well in East-Central Texas, U.S.A: Palynology, v. 10, p. 225-234.

Muller, P.A., Heatherington, A.L., Wooden, J.L., Shuster, R.D., and Nutman, A.P., 1994, Precambrian Zircons from the Florida Basement: A Gondwanan Connection; Geology, v. 22, p. 199-122.

Mueller, P., Heatherington, A.L., Foster, D.A., Thomas, W.A., and Wooden, J.L., 2014, The Suwannee Suture: Significance for Gondwana-Laurentia Terrane Transfer and Formation of Pangea: Gondwana Research, v. 26, p. 365-373.

Mueller, P.A., Michel, V., Foster, D.A., Heatherington, A.L., and Thomas, W.A., 2016, The Suwannee and Uchee Terranes: Examples of Incongruous Alleghanian Accretion in the Southern Appalachians: Geological Society of America Abstracts with Programs, v. 48, no. 3.

Odom, A.L., and Fullagar, P.D., 1973, Geochronologic and Tectonic Relationships Between the Inner Piedmont, Brevard Zone, and Blue Ridge Belts, North Carolina: American Journal of Science, v. 273, p. 133-149.

Odom, A.L., and Fullagar, P.D., 1984, Rb-Sr Whole-Rock and Inherited Zircon Ages of the Plutonic Suite of the Crossnore Complex, Southern Appalachians, and Their Implications Regarding the Time of the Opening of the Iapetus Ocean, v. 194, p. 255-262.

Ownby, S.E, Miller, C.F., Berquist, P.J., Carrigan, C.W., Wooden, J.L., and Fullagar, P.D, 2004, $\mathrm{U}-\mathrm{Pb}$ Geochronology and Geochemistry of a Portion of the Mars Hill Terrane, North Carolina- 
Tennessee: Constraints on Origin, History, and Tectonic Assembly, in Tollo, R.P., Corriveau, L., McLessland, J., and Bartholomew, M.J., eds., Proterozoic Tectonic Evolution of the Grenville Orogen in North America: Geological Society of America Memoir, v. 197, p. 609-632.

Park, H., Barbeau, D.L., Rickenbaker, A., Bachmann-Krug, D., and Gehrels, G., 2010, Application of Foreland Basin Detrital-Zircon Geochronology to the Reconstruction of the Southern and Central Appalachian Orogen: Journal of Geology, v. 118, p. 23-44.

Parker, E.H., 2014, Crustal Magnetism, Tectonic Inheritance, and Continental Rifting in the Southeastern United States: GSA Today, v. 24, p. 4-9.

Paton, C., Woodhead, J.D., Hellstrom, J.C., Hergt, J.M., Grieg, A., and Maas, R., 2010, Improved Laser Ablation U-Pb Zircon Geochronology Through Robust Downhole Fractionation Correction: Geochemistry, Geophysics, Geosystems, v. 11, p. 1-36.

Pindell, J.L., and Kennan, L., 2009, Tectonic Evolution of the Gulf of Mexico, Caribbean, and Northern South America in the Mantle Reference Frame: An Update: Geological Society of London Special Publication, v. 328, p. 1-55.

Pollastro, R.M., Schenk, C.J., and Charpentier, R.R., 2001, Assessment of Undiscovered Oil and Gas in the Onshore and State Waters Portion of the South Florida Basin: Florida-USGS Province 50, U.S Geological Survey Digital Data Series 69-A.

Pojeta, J., Kriz, J., and Berdan, J.M, 1976, Silurian-Devonian Pelecypods and Paleozoic Stratigraphy and Structure of Subsurface Rocks in Florida and Georgia and Related Silurian Pelecypods from Bolivia and Turkey: U.S Geological Survey Professional Paper 879, 32 p.

Pollock, J., Hibbard, J.P, and Van Stall, C.R., 2012, A Paleogeographical Review of the PeriGondwanan Realm of the Appalachian Orogen: Canadian Journal of Earth Sciences, v. 49, p. 259-288.

Presley, M.W., and Reed, C.H., 1984, Jurassic Exploration Trends of East Texas, in M.W. Preseley, eds., The Jurassic of East Texas: East Texas Geological Society, p. 11-22.

Rahl, J.M., Reiners, P.W., Campbell, I.H., Nicolescu, S., and Allen, C.M., 2003, Combined Single-Grain (U-Th)/He and U/Pb Dating of Detrital Zircons from the Navajo Sandstone, Utah: Geology, v. 31, p. 761-764.

Ramos, V.A., and Kay, S.M., 1991, Triassic Rifting and Associated Basalts in the Cuyo Basin, Central Argentina: The Geological Society of America Special Paper v. 265, p. 79-91.

Rhodes, J.A., and Maxwell, G.B., 1993, Jurassic Stratigraphy of the Wiggins Arch, Mississippi: Gulf Coast Association of Geological Societies Transactions, v. 43, p. 333-344. 
Rine, J.M., 2014, Reconstruction of Diagenetic and Burial History of South Georgia Rift BasinAnalysis of Sandstones from the Rizer \#1 South Carolina: Houston Geological Survey, Houston, TX, 10, November, 2014.

Rivers, T., 2008, Assembly and Preservation of Lower, Mid, and Upper Orogenic Crust in the Grenville Province-Implications for the Evolution of Large Hot Long-Duration Orogens:

Precambrian Research, v. 167, p. 237-259.

Rivers, T., Martignole, J., Gower, C., and Davidson, A., 1989, New Tectonic Divisions of the Grenville Province, Southeast Canadian Shield: Tectonics, v. 8, p. 63-84.

Rojas, P., The Road to Shell's Appomattox Discovery, Gulf Coast Association of Geological Societies, Houston TX, September 20-22, 2015

Rojas-Agramonte, Y.A, Kröner, A.J, Pindell, J.A, Casco-García, A.D., García-Delgado, D.D., Liu, D.Y., and Wang, Y.L., 2008, Detrital Zircon Geochronology of Jurassic Sandstones of Western Cuba (San Cayetano Formation): Implications for the Jurassic Paleogeography of the NW Proto-Caribbean: American Journal of Science, v. 308, p. 639-656.

Salda, L.H.D., Dalziel, I.W.D., Cingolani, C.A., and Varela, R., 1991, Did the Taconic Appalachians Continue into Southern South America: v. 20, p. 1059-1062.

Salda, L.H.D., Luchi, M.G.L., Congolani, C.A., and Varela, R., 1998, Laurentia-Gondwana Collision: The Origin of the Famatinian-Appalachian Orogenic Belt (a review): The Geological Society, v. 142, p. 219-234.

Salvador, A., 1987, Late Triassic-Jurassic Paleogeography and Origin of Gulf of Mexico Basin: Paleogeography and Origin of Gulf of Mexico Basin: v. 71, p. 419-451.

Samson, S.D., and Secor, D.T., 2001, Wandering Carolina: Tracking Exotic Terranes with Detrital Zircons, GSA Abstracts with Programs, v. 33, p. A-263.

Sartain, S.M., and See, B.E., 1997, The South Georgia Basin: An Integration of Landsat, Gravity, Magnetics, and Seismic Data to Delineate Basement Structure and Rift Basin Geometry: Gulf Coast Association of Geological Society Transactions, v. 47, p. 493-498.

Schlische, R.W., Withjack, M.O., and Olsen, P.E., 2003, Relative Timing of CAMP Rifting Continental Breakup, and Basin Inversion: Tectonic Significance., in Hames, W.E., McHone, J.G., Renne, P.R., and Ruppel, C., eds., The Central Atlantic Magmatic Province Insights from Fragments of Pangea: American Geophysical Union Monograph, p. 33-59.

Schwartz, J.J., Johnson, K., and Ingram, S., 2011, U-Pb Zircon Geochronology of Neoacadian and Early Alleghanian Plutonic Rocks in the Alabama Eastern Blue Ridge, Southern Appalachian Mountains: Geological Society of America Abstracts with Programs, v. 43, p. 62. 
Shillington, D.J., Lizarralde, D., Harder, S.H., Initial Constraints on Crustal Structure Across the Suwannee Suture and South Georgia Basin from the SUGAR Seismic Refraction Experiment: American Geophysical Union, San Francisco, CA, December 15-19, 2014.

Sinha, A.K., Thomas, W.A., Hatcher, R.D., and Harrison, T.M., 2012, Geodynamic Evolution of the Central Appalachian Orogen: Geochronology and Compositional Diversity of Magmatism from Ordovician Through Devonian: American Journal of Science, v. 312, p. 907-966.

Royal Dutch Shell, 2014, Sell and the Norphlet Play, http://s07.static shell.com/content/dam/royaldutchshell/documents/corporate/shell-norphlet-playfact-sheet.pdf (accessed September, 2016)

Schofield, D.I., and Gillespie, M.R., 2007, A Tectonic Interpretation of "Eburnean Terrane" Outliers in the Reguibat Shield, Mauritania: Journal of African Earth Sciences, v. 49, p. 179-186.

Smoot, J.P., and Olsen, P.E., 1988, Massive Mudstones in Basin Analysis and Paleoclimatic Interpretation of the Newark Supergroup: W. Manspeizer, Triassic-Jurassic Rifting and the Opening of the Atlantic Ocean: Elsevier, Amsterdam, p. 249-274.

Solari, L.A., Keppie, J.D., Ortega-Gutiérrez, F., Cameron, K.L., Lopez, R., and Hames, W.E., 2003, 990 and 1100 Ma Grenvillian Tectonothermal Events in the Northern Oaxacan Complex, Southern Mexico: Roots of an Orogen: Tectonophysics, v. 365, p. 257-282.

Speer, J.A., McSween, H.Y., Jr., and Gates, A.E., 1994, Generation, Segregation, Ascent, and Emplacement of Alleghanian Plutons in the Southern Appalachians: The Journal of Geology, v. 102, p. 249-267.

Steiner, M.B., and Walker, J.D., 1996, Late Silurian Plutons in Yucatan: Journal of Geophysical Research, v. 101, p. 127-735.

Steltenpohl, M.G., Hatcher Jr., R.D., Mueller, P.A., Heatherington, A.L., and Wooden, J.L., 2010, Geologic History of the Pine Mountain Window, Alabama and Georgia: Insights from a new Geologic Map and U-Pb isotopic Dates., in Tollo, R.P., Bartholomew, M.J., Hibbard, J.P., and Karabinos, P.M., eds., From Rodinia to Pangea: The Lithospheric Record of the Appalachian Region: The Geological Society of America Memoir v. 206, p. 837-857.

Steltenpohl, M.G., Horton, W., Hatcher, R.D., Zietz, I., Daniels, D.L., and Higgins, M.W., 2013, Upper Crustal Structure of Alabama from Regional Magnetic and Gravity Data: Using Geology to Interpret Geophysics, and Vice Versa: Geological Society of America, v. 9, p. 1044-1064.

Steltenpohl, M.G., Mueller, P.M., Heatherington, A.L., Hanley, T.B., and Wooden, J.L., 2008, Gondwanan/Peri-Gondwanan Origin for the Uchee Terrane, Alabama and Georgia: Carolina Zone or Suwannee Terrane (?) and its Suture with Grenvillian Basement of the Pine Mountain Window: v. 4, p. 131-144.

Stern, J.R., and Dickinson, W.R., 2010, The Gulf of Mexico is A Jurassic Backarc Basin: Geosphere, v. 6, p. 739-754. 
Tew, B.H., Mink, R.N., Mann, S.D., Bearden, B.L., and Mancini, E.A., 1991, Geologic Framework of Norphlet and Pre-Norphlet Strata of the Onshore and Offshore Eastern Gulf of Mexico Area: Gulf Coast Association of Geological Societies Transactions, v. 41, p. 590-600.

Thomas, W.A., 1989, The Appalachian-Ouachita Orogen Beneath the Gulf Coastal Plain Between the Outcrops in the Appalachian and Ouachita Mountains, in Hatcher, R.D., Thomas, W.A., and Viele, G.W., eds., The Appalachian-Ouachita Orogen in the United States: Geological Society of America, The Geology of North America, v. F-2, p. 527-553.

Thomas, W, A., 2010, Interactions Between the Southern Appalachian-Ouachita Orogenic Belt and Basement Faults in the Orogenic Footwall and Foreland, in Tollo, R.P., Bartholomew, M.J., Hibbard, J.P., and Karabinos, P.M., eds., From Rodinia to Pangea: The Lithospheric Record of the Appalachian Region: The Geological Society of America Memoir, v. 206, p. 897-916

Thomas, W.A., Becker, T.P., Samson, S.D., and Hamilton, M.A, 2004, Detrital Zircon Evidence of a Recycled Orogenic Foreland Provenance for Alleghanian Clastic-Wedge Sandstone: Journal of Geology, v. 112, p. 23-37.

Tohver, E., Teixeira, W., Van der Pluijm, B., Geraldes, M.C., Bettencourt, J.S., and Rizzotto, G., 2016, Restored Transect Across the Exhumed Grenville Orogen of Laurentia and Amazonia, With Implications for Crustal Architecture, Geological Society of America, v. 34, p. 669-672.

Warzeski, E.R., Cunningham, K.J., Ginsburg, R.N., Anderson, J.B., Ding, and Z.-D. Ding., 1996, A Neogene Mixed Siliciclastic and Carbonate Foundation for the Quaternary Carbonate Shelf, Florida Keys: Journal of Sedimentary Research, v. 66, p. 788-800.

Weber, B., and Köhler, H., 1999, Sm-Nd, Rb-Sr, and U-Pb Geochronology of a Grenville Terrane in Southern Mexico: Origin and Geologic History of the Guichicovi Complex: Precambrian Research, v. 96, p. 245-262.

Weber, B., Schaaf, P., Valencia V.A., Iriondo, A., and Ortega-Gutierrez, F., 2006, Provenance Ages of Late Paleozoic Sandstones (Santa Rosa Formation) from the Maya block, SE Mexico: Implications on the Tectonic Evolution of Western Pangea: Revista Mexicana de Ciencias Geologicas, v. 23, p. 262-276.

Weber, B., Valencia, V.A., Schaaf, P., G., and Ortega-Gutierrez, F., 2009, Detrital Zircon Ages from the Lower Santa Rosa Formation, Chiapas: Implications on Regional Paleozoic Stratigraphy: Revista Mexicana de Ciencias Geologicas, v. 26, p. 260-276.

Weislogel, A.L., Hunt, B., Lisi, A., Lovell, T., and Robinson, D.M., 2015, Detrital Zircon Provenance of the Eastern Gulf of Mexico Subsurface: Constraints on Late Jurassic Paleogeography and Sediment Dispersal of North America., in Anderson, T.H., Didenko, A.N., Johnson, C.L., Khanchuk, A.I., and MacDonald, J.H., Jr., eds., Late Jurassic Margin of Laurasia-A Record of Faulting Accommodating Plate Rotation: Geological Society of America Special Paper 513 
Widenbeck, M., Allé, P., Corfu, F., Griffin, W.L., Meier, M., Oberli, F., von Quadt, A., Roddick, J.C., and Siegel, W., 1995, 3 Natural Zircon Standards for U-Th-Pb, Lu-Hf, Trace-Element and REE Analysis: Geostandards Newsletter, v. 19, p. 1-23.

Williams, H., and Hatcher, R.D., 1983, Appalachian Suspect Terranes., in Hatcher, R.D., Williams, H., and Zietz, I., eds., Contributions to the Tectonics and Geophysics of Mountain Chains, v. 158, p. 33-53.

Wilson, J.R., 2001, U/Pb Ages of Plutons from the Central Appalachians and GIS-Based Assessment of Plutons with Comments on Their Regional Tectonic Significance, Master's thesis, Virginia Technical University, p. 109

Wilson, K.L., 2012, The Origin and Development of the Tampa Embayment: Implications for the Tectonic Evolution of the Eastern Gulf of Mexico: The University of Alabama, Master's thesis, p. 63.

Withjack, M.O., Schlische, R.W., Olsen, P.E., 1998, Diachronous Rifting, Drifting, and Inversion on the Passive Margin of Central Eastern North America: An Analog for Other Passive Margins; v. 82, p. 817-835.

World Oil, 2016, Shell Reports New Deepwater Discovery in U.S Gulf of Mexico: http://www.worldoil.com/news/2016/7/28/shell-reports-new-deepwater-discovery-in-us-gulf-of$\underline{\text { mexico }}$ (accessed September, 2016)

Wortman, G.L., Samson, S.D., and Hibbard, J.P., 2000, Precise U-Pb Zircon Constraints on the Earliest Magmetic History of the Carolina Terrane: The Journal of Geology, v.108, p. 321-338.

Van der Plas, L., and Tobi, A.C., 1965, A Chart for Judging the Reliability of Point Counting Results: American Journal of Science, v. 263, p. 87-90.

Vermeesch, P., 2012, On the Visualization of Detrital Age Distributions: Chemical Geology, v. 312-313, p. 190-194.

Ziegler, D.G., 1983, Hydrocarbon Potential of the Newark Rift System: Eastern North America: Northeastern Geology, v. 5, p. 200-208. 


\section{Appendix A: U-Pb data}

\begin{tabular}{|c|c|c|c|c|c|c|c|c|c|c|c|c|c|c|c|c|c|}
\hline Analysis & $\begin{array}{l}\text { U } \\
\text { pp } \\
\mathrm{m}\end{array}$ & $\begin{array}{l}\text { Th } \\
\text { ppm }\end{array}$ & U/Th & $\begin{array}{l}207 \mathrm{~Pb} / 2 \\
35 \mathrm{U}\end{array}$ & $\begin{array}{l}+/- \\
2 \sigma\end{array}$ & $\begin{array}{l}206 \mathrm{~Pb} / 2 \\
38 \mathrm{U}\end{array}$ & $\begin{array}{l}+/- \\
2 \sigma\end{array}$ & $\begin{array}{l}207 P \\
\text { b/ } \\
206 P \\
\text { b }\end{array}$ & $\begin{array}{l}+/- \\
2 \sigma\end{array}$ & $\begin{array}{l}\text { 207P } \\
\text { b/ } \\
235 \\
\mathrm{U} \\
\text { (Ma) }\end{array}$ & $\begin{array}{l}+/ \\
- \\
\sigma \\
M \\
a\end{array}$ & $\begin{array}{l}206 \mathrm{~Pb} / 2 \\
35 \mathrm{U} \\
(\mathrm{Ma})\end{array}$ & $\begin{array}{l}+/ \\
- \\
\sigma \\
M \\
a\end{array}$ & $\begin{array}{l}\text { 207P } \\
\text { b/ } \\
206 P \\
\text { b } \\
\text { (Ma) }\end{array}$ & $\begin{array}{l}+/ \\
- \\
\sigma \\
M \\
a\end{array}$ & $\begin{array}{l}\text { Bes } \\
\mathrm{t} \\
\mathrm{Ag} \\
\mathrm{e} \\
\mathrm{Ma}\end{array}$ & $\begin{array}{l}\text { Use } \\
\text { d? }\end{array}$ \\
\hline \multicolumn{18}{|l|}{3578} \\
\hline X3578_1 & 225 & 16.3 & 14.4 & 0.773 & $\begin{array}{l}0.0 \\
56\end{array}$ & 0.0958 & $\begin{array}{l}0.00 \\
58\end{array}$ & $\begin{array}{l}0.05 \\
94\end{array}$ & $\begin{array}{l}0.00 \\
23\end{array}$ & 589 & 16 & 589 & 17 & 561 & 42 & 589 & yes \\
\hline X3578_2 & 257 & 86 & 3.15 & 0.913 & $\begin{array}{l}0.0 \\
68 \\
\end{array}$ & 0.1028 & $\begin{array}{l}0.00 \\
55 \\
\end{array}$ & $\begin{array}{l}0.06 \\
48 \\
\end{array}$ & $\begin{array}{l}0.00 \\
37 \\
\end{array}$ & 659 & $\begin{array}{l}18 \\
.5 \\
\end{array}$ & 630 & 16 & 740 & 60 & 630 & yes \\
\hline X3578_3 & $\begin{array}{l}38 . \\
7\end{array}$ & 7.6 & 8.2 & 3.46 & $\begin{array}{l}0.5 \\
9\end{array}$ & 0.1379 & $\begin{array}{l}0.00 \\
92\end{array}$ & $\begin{array}{l}0.16 \\
7\end{array}$ & $\begin{array}{l}0.02 \\
3\end{array}$ & 1450 & 60 & 831 & 26 & 2410 & $\begin{array}{l}11 \\
0\end{array}$ & 831 & no \\
\hline X3578_4 & 129 & 31.8 & 3.96 & 0.8 & $\begin{array}{l}0.0 \\
49\end{array}$ & 0.098 & $\begin{array}{l}0.00 \\
48\end{array}$ & $\begin{array}{l}0.05 \\
8\end{array}$ & $\begin{array}{l}0.00 \\
19\end{array}$ & 593 & $\begin{array}{l}13 \\
.5\end{array}$ & 602 & 14 & 540 & 37 & 602 & yes \\
\hline X3578_5 & 180 & 55.6 & 3.14 & 0.874 & $\begin{array}{l}0.0 \\
54 \\
\end{array}$ & 0.094 & $\begin{array}{l}0.00 \\
48 \\
\end{array}$ & $\begin{array}{l}0.06 \\
72 \\
\end{array}$ & $\begin{array}{l}0.00 \\
3\end{array}$ & 634 & $\begin{array}{l}14 \\
.5 \\
\end{array}$ & 579 & 14 & 832 & 48 & 579 & yes \\
\hline X3578_7 & 163 & 77.8 & 2.13 & 0.842 & $\begin{array}{l}0.0 \\
33\end{array}$ & 0.0974 & $\begin{array}{l}0.00 \\
3 \\
\end{array}$ & $\begin{array}{l}0.06 \\
21 \\
\end{array}$ & $\begin{array}{l}0.00 \\
21 \\
\end{array}$ & 619 & 9 & 599 & 9 & 661 & $\begin{array}{l}35 \\
.5\end{array}$ & 599 & yes \\
\hline X3578_8 & $\begin{array}{l}82 . \\
3\end{array}$ & 47.1 & 1.78 & 1.69 & $\begin{array}{l}0.1 \\
3 \\
\end{array}$ & 0.0997 & $\begin{array}{l}0.00 \\
71 \\
\end{array}$ & $\begin{array}{l}0.12 \\
19 \\
\end{array}$ & $\begin{array}{l}0.00 \\
64 \\
\end{array}$ & 1012 & $\begin{array}{l}25 \\
.5 \\
\end{array}$ & 619 & 20 & 1975 & $\begin{array}{l}48 \\
.5 \\
\end{array}$ & 619 & yes \\
\hline X3578_9 & $\begin{array}{l}72 . \\
5 \\
\end{array}$ & 17.9 & 4.04 & 0.773 & $\begin{array}{l}0.0 \\
36 \\
\end{array}$ & 0.0935 & $\begin{array}{l}0.00 \\
43 \\
\end{array}$ & $\begin{array}{l}0.05 \\
84 \\
\end{array}$ & $\begin{array}{l}0.00 \\
2 \\
\end{array}$ & 580 & 10 & 576 & $\begin{array}{l}12 \\
.5\end{array}$ & 556 & $\begin{array}{l}36 \\
.5\end{array}$ & 576 & yes \\
\hline $\begin{array}{l}\text { X3578_1 } \\
0\end{array}$ & $\begin{array}{l}195 \\
.5 \\
\end{array}$ & 42.1 & 4.44 & 0.877 & $\begin{array}{l}0.0 \\
45 \\
\end{array}$ & 0.0928 & $\begin{array}{l}0.00 \\
35 \\
\end{array}$ & $\begin{array}{l}0.06 \\
78 \\
\end{array}$ & $\begin{array}{l}0.00 \\
32\end{array}$ & 637 & 12 & 576 & 10 & 870 & 50 & 576 & yes \\
\hline $\begin{array}{l}\text { X3578_1 } \\
1\end{array}$ & 89 & 25.4 & 3.63 & 0.803 & $\begin{array}{l}0.0 \\
94 \\
\end{array}$ & 0.105 & $\begin{array}{l}0.01 \\
1 \\
\end{array}$ & $\begin{array}{l}0.05 \\
66 \\
\end{array}$ & $\begin{array}{l}0.00 \\
31 \\
\end{array}$ & 597 & $\begin{array}{l}27 \\
.5 \\
\end{array}$ & 640 & 32 & 480 & 65 & 640 & no \\
\hline $\begin{array}{l}\text { X3578_1 } \\
2\end{array}$ & $\begin{array}{l}62 . \\
5 \\
\end{array}$ & 25.5 & 2.56 & 0.802 & $\begin{array}{l}0.0 \\
45 \\
\end{array}$ & 0.0918 & $\begin{array}{l}0.00 \\
44 \\
\end{array}$ & $\begin{array}{l}0.06 \\
24 \\
\end{array}$ & $\begin{array}{l}0.00 \\
24\end{array}$ & 595 & $\begin{array}{l}12 \\
.5 \\
\end{array}$ & 565 & 13 & 694 & $\begin{array}{l}41 \\
.5 \\
\end{array}$ & 565 & yes \\
\hline $\begin{array}{l}\text { X3578_1 } \\
3\end{array}$ & 131 & 86 & 1.59 & 0.843 & $\begin{array}{l}0.0 \\
5 \\
\end{array}$ & 0.0887 & $\begin{array}{l}0.00 \\
33 \\
\end{array}$ & $\begin{array}{l}0.06 \\
89 \\
\end{array}$ & $\begin{array}{l}0.00 \\
3 \\
\end{array}$ & 617 & $\begin{array}{l}13 \\
.5 \\
\end{array}$ & 547 & $\begin{array}{l}9 . \\
5 \\
\end{array}$ & 872 & 44 & 547 & yes \\
\hline $\begin{array}{l}\text { X3578_1 } \\
4\end{array}$ & $\begin{array}{l}100 \\
.7 \\
\end{array}$ & 40.8 & 2.4 & 0.75 & $\begin{array}{l}0.0 \\
35 \\
\end{array}$ & 0.0905 & $\begin{array}{l}0.00 \\
37 \\
\end{array}$ & $\begin{array}{l}0.06 \\
11 \\
\end{array}$ & $\begin{array}{l}0.00 \\
19 \\
\end{array}$ & 566 & 10 & 558 & 11 & 655 & $\begin{array}{l}33 \\
.5 \\
\end{array}$ & 558 & yes \\
\hline $\begin{array}{l}\text { X3578_1 } \\
6\end{array}$ & $\begin{array}{l}91 . \\
6 \\
\end{array}$ & 59 & 1.76 & 0.954 & $\begin{array}{l}0.0 \\
55\end{array}$ & 0.0998 & $\begin{array}{l}0.00 \\
35 \\
\end{array}$ & $\begin{array}{l}0.07 \\
07 \\
\end{array}$ & $\begin{array}{l}0.00 \\
38 \\
\end{array}$ & 677 & $\begin{array}{l}13 \\
.5 \\
\end{array}$ & 613 & 10 & 910 & 55 & 613 & yes \\
\hline $\begin{array}{l}\text { X3578_1 } \\
7\end{array}$ & $\begin{array}{l}95 . \\
6 \\
\end{array}$ & 11.81 & 8.4 & 0.812 & $\begin{array}{l}0.0 \\
54 \\
\end{array}$ & 0.0978 & $\begin{array}{l}0.00 \\
53 \\
\end{array}$ & $\begin{array}{l}0.06 \\
04 \\
\end{array}$ & $\begin{array}{l}0.00 \\
25 \\
\end{array}$ & 599 & 15 & 601 & $\begin{array}{l}15 \\
.5 \\
\end{array}$ & 592 & 47 & 601 & yes \\
\hline $\begin{array}{l}\text { X3578_1 } \\
8\end{array}$ & 120 & 32.9 & 3.61 & 1.019 & $\begin{array}{l}0.0 \\
75 \\
\end{array}$ & 0.0985 & $\begin{array}{l}0.00 \\
55 \\
\end{array}$ & $\begin{array}{l}0.07 \\
37 \\
\end{array}$ & $\begin{array}{l}0.00 \\
42 \\
\end{array}$ & 707 & 19 & 605 & 16 & 1020 & 55 & 605 & yes \\
\hline $\begin{array}{l}\text { X3578_1 } \\
9\end{array}$ & $\begin{array}{l}48 . \\
8\end{array}$ & 22.7 & 2.16 & 1.56 & $\begin{array}{l}0.2 \\
9\end{array}$ & 0.1001 & $\begin{array}{l}0.00 \\
51\end{array}$ & $\begin{array}{l}0.11 \\
2\end{array}$ & $\begin{array}{l}0.01 \\
7\end{array}$ & 910 & 60 & 614 & 15 & 1570 & $\begin{array}{l}14 \\
5\end{array}$ & 614 & yes \\
\hline $\begin{array}{l}\text { X3578_2 } \\
0\end{array}$ & 88 & 28.4 & 3.34 & 0.775 & $\begin{array}{l}0.0 \\
52 \\
\end{array}$ & 0.0961 & $\begin{array}{l}0.00 \\
51 \\
\end{array}$ & $\begin{array}{l}0.05 \\
87 \\
\end{array}$ & $\begin{array}{l}0.00 \\
24 \\
\end{array}$ & 584 & $\begin{array}{l}14 \\
.5 \\
\end{array}$ & 591 & 15 & 564 & $\begin{array}{l}45 \\
.5 \\
\end{array}$ & 591 & yes \\
\hline $\begin{array}{l}\text { X3578_2 } \\
1\end{array}$ & 104 & 41.8 & 2.48 & 1.033 & $\begin{array}{l}0.0 \\
61\end{array}$ & 0.1181 & $\begin{array}{l}0.00 \\
63\end{array}$ & $\begin{array}{l}0.06 \\
43\end{array}$ & $\begin{array}{l}0.00 \\
28\end{array}$ & 716 & $\begin{array}{l}15 \\
.5\end{array}$ & 718 & $\begin{array}{l}18 \\
.5\end{array}$ & 727 & $\begin{array}{l}45 \\
.5\end{array}$ & 718 & yes \\
\hline $\begin{array}{l}\text { X3578_2 } \\
2\end{array}$ & $\begin{array}{l}94 . \\
7 \\
\end{array}$ & 44.4 & 2.26 & 0.823 & $\begin{array}{l}0.0 \\
68 \\
\end{array}$ & 0.0957 & $\begin{array}{l}0.00 \\
6 \\
\end{array}$ & $\begin{array}{l}0.06 \\
27 \\
\end{array}$ & $\begin{array}{l}0.00 \\
33 \\
\end{array}$ & 603 & 19 & 588 & $\begin{array}{l}17 \\
.5 \\
\end{array}$ & 690 & 55 & 588 & yes \\
\hline $\begin{array}{l}\text { X3578_2 } \\
4\end{array}$ & $\begin{array}{l}112 \\
.9\end{array}$ & 38.3 & 3.07 & 5.48 & 0.4 & 0.316 & $\begin{array}{l}0.01 \\
8\end{array}$ & $\begin{array}{l}0.12 \\
76\end{array}$ & $\begin{array}{l}0.00 \\
58\end{array}$ & 1888 & 32 & 1762 & 43 & 2074 & $\begin{array}{l}39 \\
.5\end{array}$ & $\begin{array}{l}207 \\
4\end{array}$ & yes \\
\hline $\begin{array}{l}\text { X3578_2 } \\
5\end{array}$ & 319 & 99 & 3.28 & 1.006 & $\begin{array}{l}0.0 \\
7 \\
\end{array}$ & 0.1118 & $\begin{array}{l}0.00 \\
6 \\
\end{array}$ & $\begin{array}{l}0.06 \\
72 \\
\end{array}$ & $\begin{array}{l}0.00 \\
33 \\
\end{array}$ & 714 & $\begin{array}{l}17 \\
.5 \\
\end{array}$ & 682 & $\begin{array}{l}17 \\
.5 \\
\end{array}$ & 835 & $\begin{array}{l}47 \\
.5 \\
\end{array}$ & 682 & yes \\
\hline $\begin{array}{l}\text { X3578_2 } \\
6\end{array}$ & $\begin{array}{l}81 . \\
7\end{array}$ & 38.7 & 2.23 & 1.045 & $\begin{array}{l}0.0 \\
57\end{array}$ & 0.0979 & $\begin{array}{l}0.00 \\
5\end{array}$ & $\begin{array}{l}0.07 \\
55\end{array}$ & $\begin{array}{l}0.00 \\
22\end{array}$ & 731 & 15 & 602 & $\begin{array}{l}14 \\
.5\end{array}$ & 1076 & 30 & 602 & yes \\
\hline $\begin{array}{l}\text { X3578_2 } \\
7\end{array}$ & $\begin{array}{l}40 . \\
8 \\
\end{array}$ & 17.3 & 2.52 & 2.67 & $\begin{array}{l}0.2 \\
4 \\
\end{array}$ & 0.229 & $\begin{array}{l}0.01 \\
5 \\
\end{array}$ & $\begin{array}{l}0.08 \\
36 \\
\end{array}$ & $\begin{array}{l}0.00 \\
41 \\
\end{array}$ & 1302 & $\begin{array}{l}32 \\
.5 \\
\end{array}$ & 1327 & 39 & 1272 & $\begin{array}{l}49 \\
.5 \\
\end{array}$ & $\begin{array}{l}132 \\
7 \\
\end{array}$ & yes \\
\hline $\begin{array}{l}\text { X3578_2 } \\
8\end{array}$ & 173 & 53 & 3.22 & 1.4 & $\begin{array}{l}0.1 \\
6\end{array}$ & 0.139 & $\begin{array}{l}0.01 \\
6\end{array}$ & $\begin{array}{l}0.07 \\
57\end{array}$ & $\begin{array}{l}0.00 \\
37\end{array}$ & 880 & $\begin{array}{l}35 \\
.5\end{array}$ & 832 & $\begin{array}{l}45 \\
.5\end{array}$ & 1073 & $\begin{array}{l}47 \\
.5\end{array}$ & 832 & yes \\
\hline $\begin{array}{l}\text { X3578_2 } \\
9\end{array}$ & $\begin{array}{l}90 . \\
3\end{array}$ & 23.3 & 4.05 & 0.833 & $\begin{array}{l}0.0 \\
56 \\
\end{array}$ & 0.097 & $\begin{array}{l}0.00 \\
54 \\
\end{array}$ & $\begin{array}{l}0.06 \\
29 \\
\end{array}$ & $\begin{array}{l}0.00 \\
34\end{array}$ & 611 & 15 & 596 & 16 & 670 & 55 & 596 & yes \\
\hline $\begin{array}{l}\text { X3578_3 } \\
0\end{array}$ & 30 & 10.73 & 2.88 & 0.772 & $\begin{array}{l}0.0 \\
6\end{array}$ & 0.0958 & $\begin{array}{l}0.00 \\
61\end{array}$ & $\begin{array}{l}0.06 \\
08\end{array}$ & $\begin{array}{l}0.00 \\
38\end{array}$ & 576 & 17 & 589 & 18 & 630 & 65 & 589 & yes \\
\hline
\end{tabular}




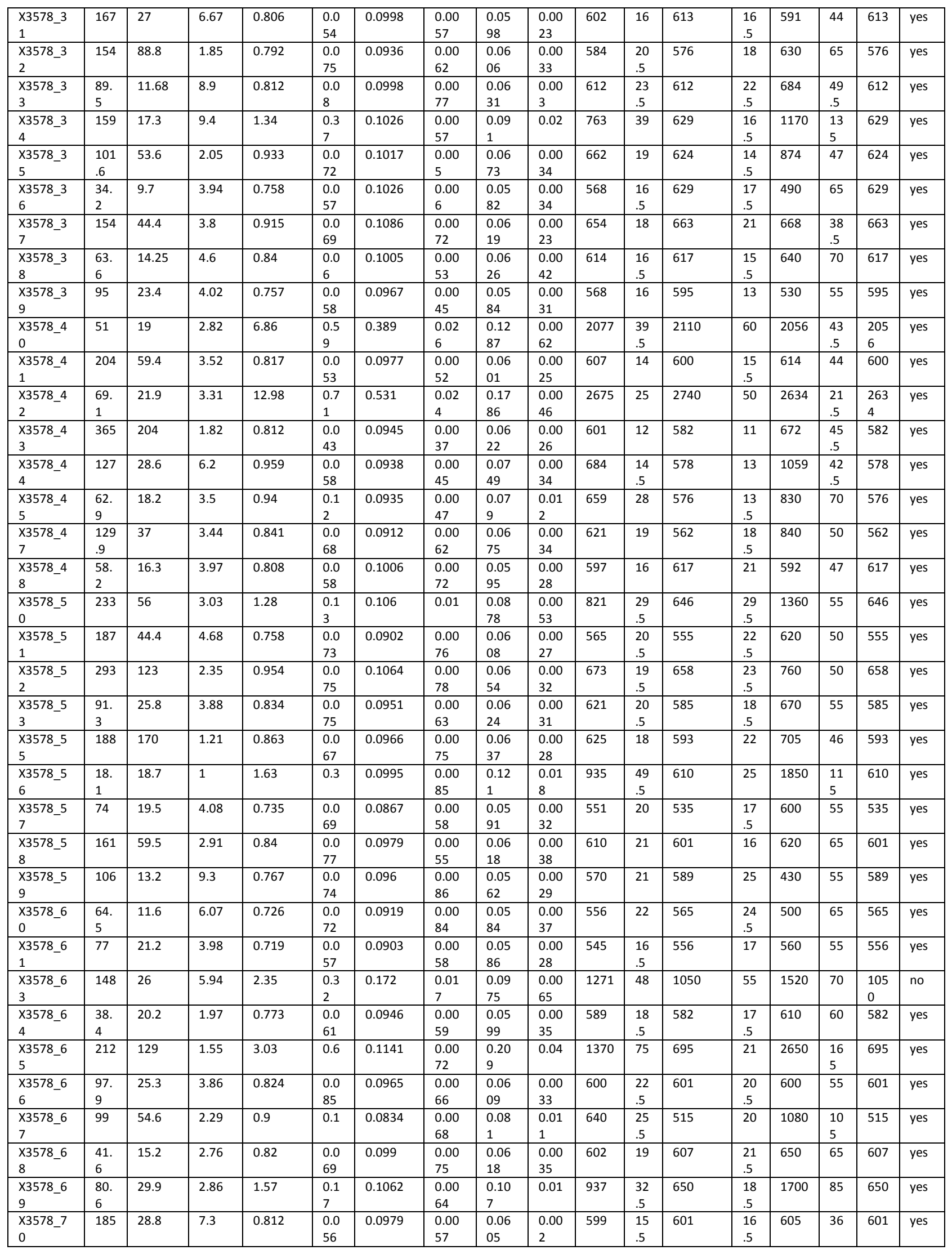




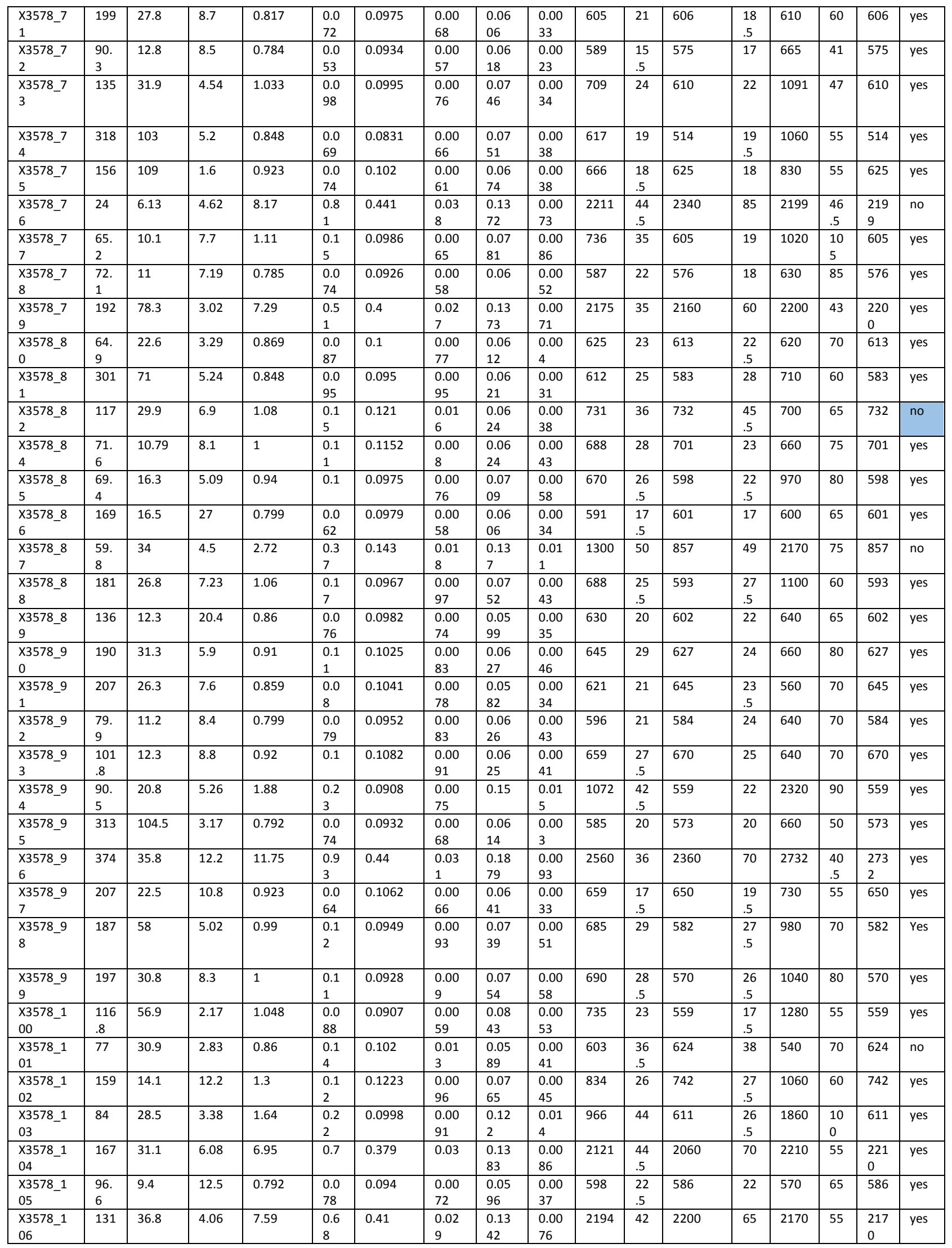




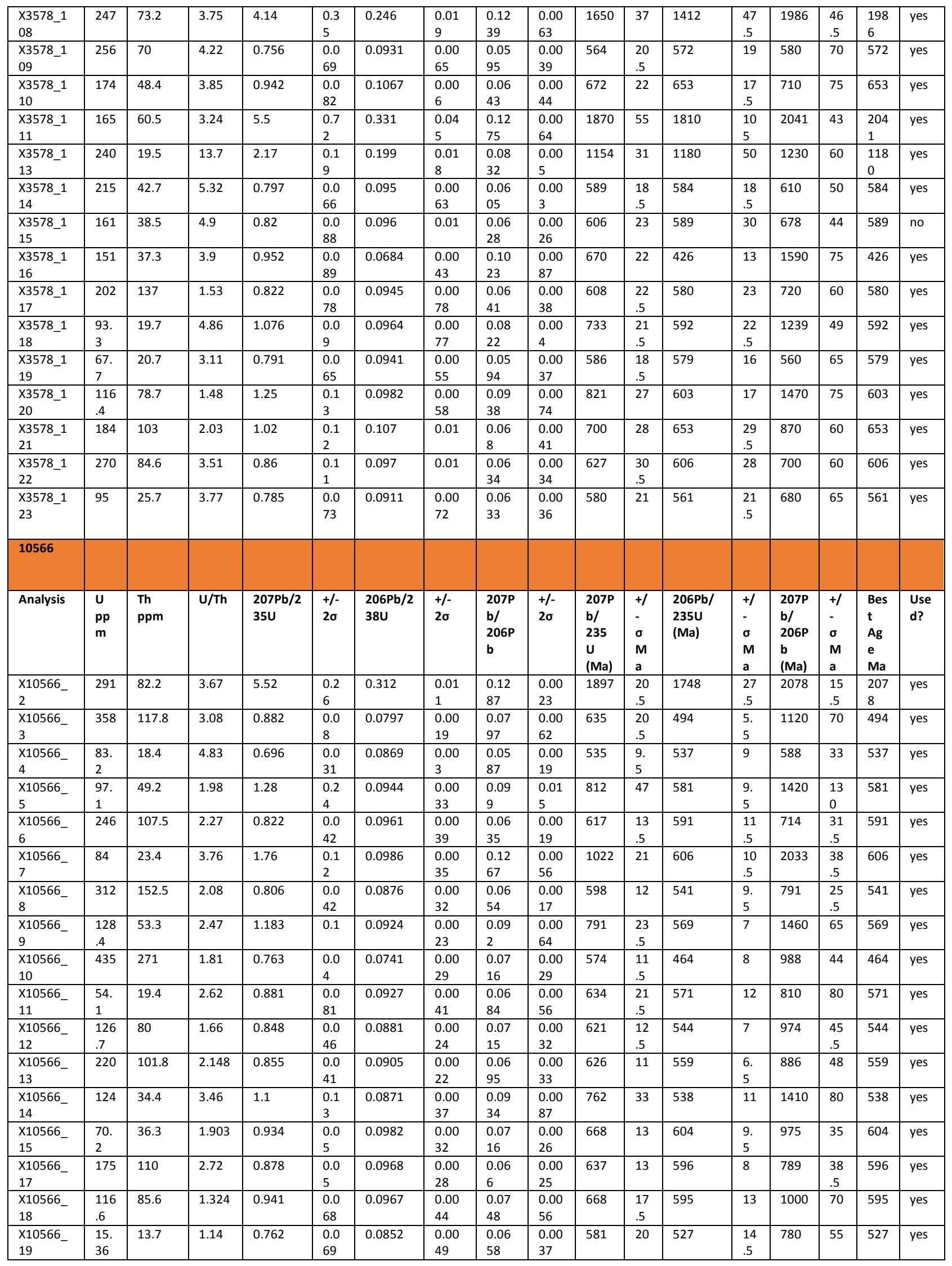




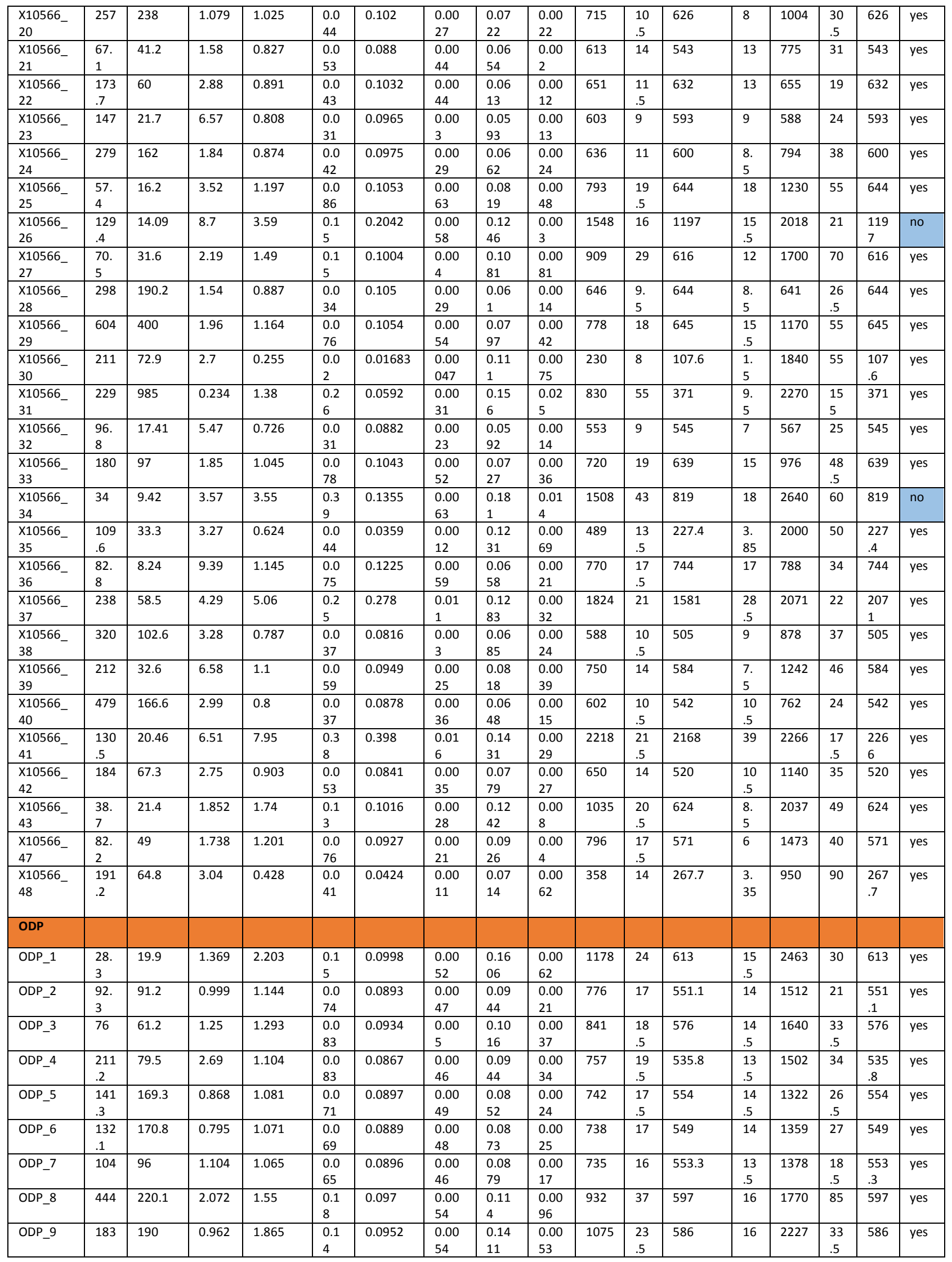




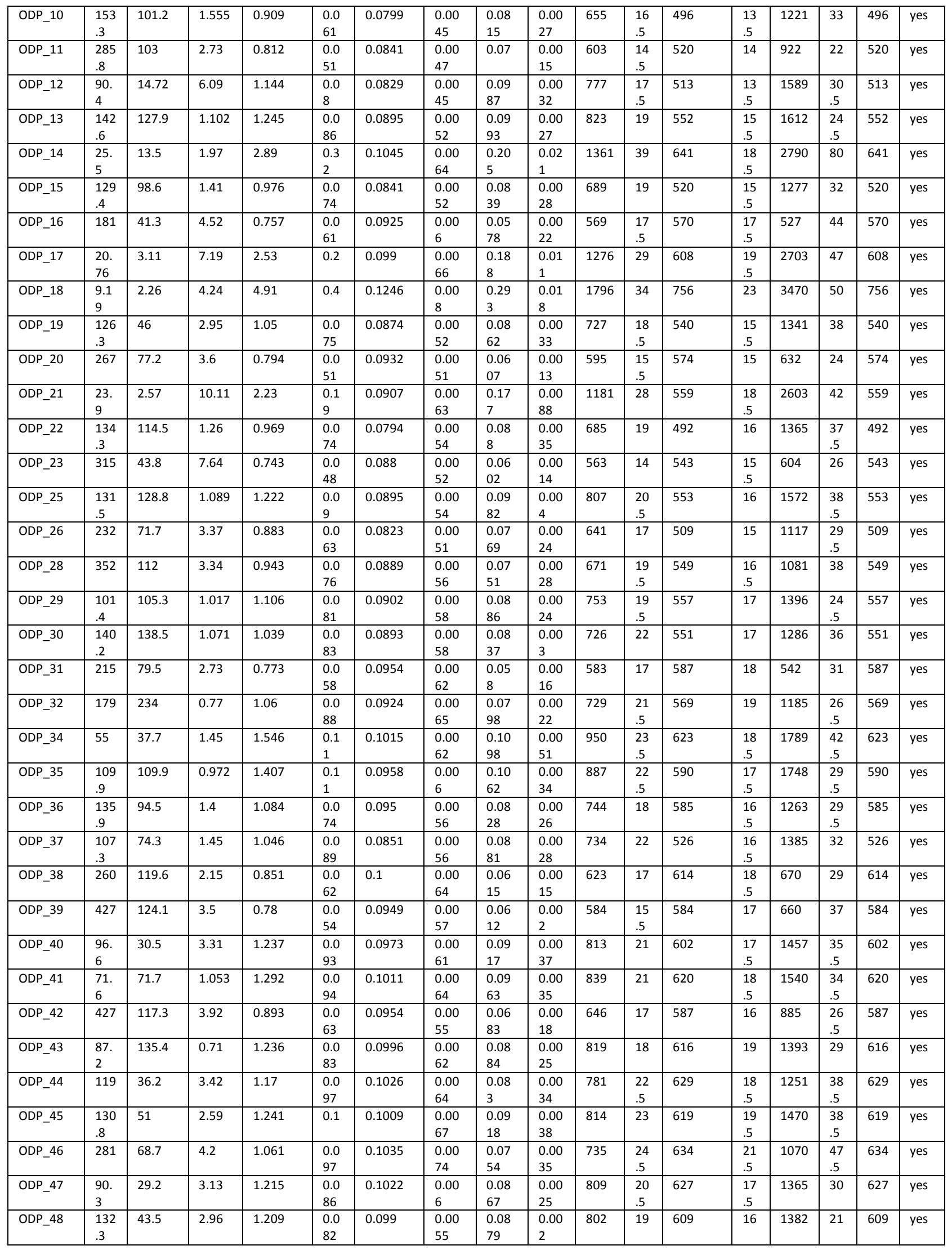




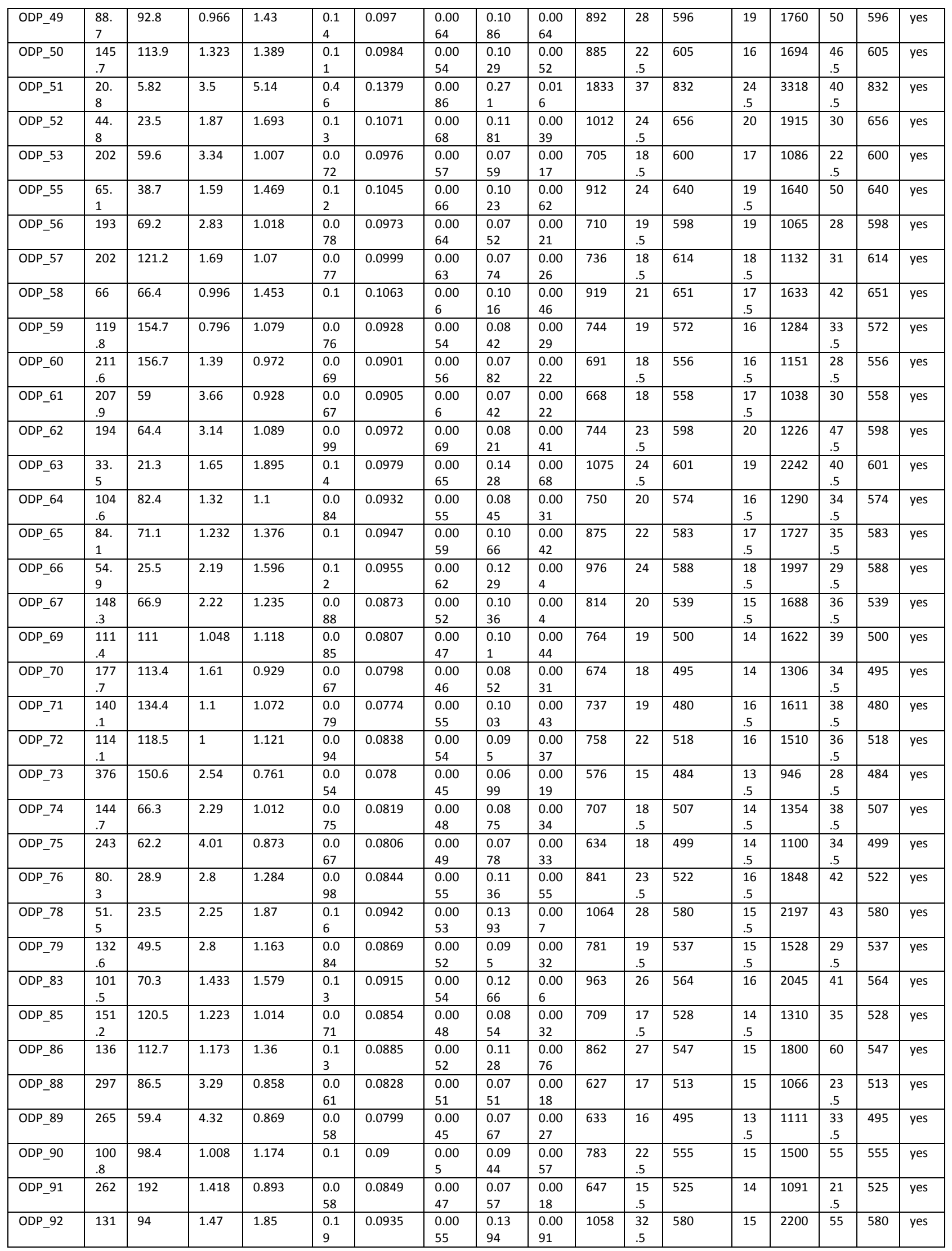




\begin{tabular}{|c|c|c|c|c|c|c|c|c|c|c|c|c|c|c|c|c|c|}
\hline ODP_93 & $\begin{array}{l}137 \\
.9\end{array}$ & 142.1 & 1.005 & 0.965 & $\begin{array}{l}0.0 \\
7\end{array}$ & 0.0824 & $\begin{array}{l}0.00 \\
5\end{array}$ & $\begin{array}{l}0.08 \\
33\end{array}$ & $\begin{array}{l}0.00 \\
31\end{array}$ & 683 & 18 & 513 & $\begin{array}{l}15 \\
.5\end{array}$ & 1261 & 36 & 513 & yes \\
\hline ODP_94 & $\begin{array}{l}155 \\
.6\end{array}$ & 157.6 & 1.102 & 0.913 & $\begin{array}{l}0.0 \\
6\end{array}$ & 0.079 & $\begin{array}{l}0.00 \\
45\end{array}$ & $\begin{array}{l}0.08 \\
26\end{array}$ & $\begin{array}{l}0.00 \\
2\end{array}$ & 660 & $\begin{array}{l}16 \\
.5\end{array}$ & 490 & $\begin{array}{l}13 \\
.5\end{array}$ & 1254 & 24 & 490 & yes \\
\hline ODP_96 & $\begin{array}{l}152 \\
.4\end{array}$ & 118 & 1.36 & 1.208 & $\begin{array}{l}0.0 \\
85\end{array}$ & 0.0888 & $\begin{array}{l}0.00 \\
53\end{array}$ & $\begin{array}{l}0.09 \\
74\end{array}$ & $\begin{array}{l}0.00 \\
31\end{array}$ & 802 & 19 & 548 & $\begin{array}{l}15 \\
.5\end{array}$ & 1563 & $\begin{array}{l}30 \\
.5\end{array}$ & 548 & yes \\
\hline ODP_97 & $\begin{array}{l}167 \\
.5 \\
\end{array}$ & 151.9 & 1.224 & 0.897 & $\begin{array}{l}0.0 \\
61 \\
\end{array}$ & 0.0789 & $\begin{array}{l}0.00 \\
43 \\
\end{array}$ & $\begin{array}{l}0.08 \\
22 \\
\end{array}$ & $\begin{array}{l}0.00 \\
24 \\
\end{array}$ & 652 & 17 & 489 & 13 & 1239 & $\begin{array}{l}29 \\
.5 \\
\end{array}$ & 489 & yes \\
\hline ODP_98 & $\begin{array}{l}32 . \\
3 \\
\end{array}$ & 16.28 & 2.21 & 2.71 & $\begin{array}{l}0.2 \\
2 \\
\end{array}$ & 0.0938 & $\begin{array}{l}0.00 \\
54 \\
\end{array}$ & $\begin{array}{l}0.20 \\
4 \\
\end{array}$ & $\begin{array}{l}0.01 \\
1 \\
\end{array}$ & 1321 & $\begin{array}{l}30 \\
.5 \\
\end{array}$ & 577 & 16 & 2838 & $\begin{array}{l}42 \\
.5 \\
\end{array}$ & 577 & yes \\
\hline ODP_99 & $\begin{array}{l}166 \\
.2\end{array}$ & 159.4 & 1.135 & 0.978 & $\begin{array}{l}0.0 \\
8\end{array}$ & 0.0797 & $\begin{array}{l}0.00 \\
46\end{array}$ & $\begin{array}{l}0.08 \\
71\end{array}$ & $\begin{array}{l}0.00 \\
38\end{array}$ & 688 & $\begin{array}{l}20 \\
.5\end{array}$ & 494 & 14 & 1367 & $\begin{array}{l}40 \\
.5\end{array}$ & 494 & yes \\
\hline ODP_100 & 178 & 59.9 & 3.14 & 1.015 & $\begin{array}{l}0.0 \\
65\end{array}$ & 0.0826 & $\begin{array}{l}0.00 \\
45\end{array}$ & $\begin{array}{l}0.08 \\
87\end{array}$ & $\begin{array}{l}0.00 \\
3\end{array}$ & 710 & 16 & 512 & 13 & 1370 & 27 & 512 & yes \\
\hline ODP_101 & 588 & 175 & 3.4 & 0.829 & $\begin{array}{l}0.0 \\
67\end{array}$ & 0.0848 & $\begin{array}{l}0.00 \\
55\end{array}$ & $\begin{array}{l}0.06 \\
84\end{array}$ & $\begin{array}{l}0.00 \\
31\end{array}$ & 610 & $\begin{array}{l}18 \\
.5\end{array}$ & 525 & $\begin{array}{l}16 \\
.5\end{array}$ & 858 & $\begin{array}{l}44 \\
.5\end{array}$ & 525 & yes \\
\hline ODP_102 & $\begin{array}{l}123 \\
.7\end{array}$ & 121.7 & 1.014 & 0.967 & $\begin{array}{l}0.0 \\
7\end{array}$ & 0.0762 & $\begin{array}{l}0.00 \\
47\end{array}$ & $\begin{array}{l}0.09 \\
1\end{array}$ & $\begin{array}{l}0.00 \\
41\end{array}$ & 684 & 18 & 473 & 14 & 1423 & $\begin{array}{l}41 \\
.5\end{array}$ & 473 & yes \\
\hline ODP_103 & 353 & 106.5 & 3.36 & 0.669 & $\begin{array}{l}0.0 \\
46\end{array}$ & 0.0824 & $\begin{array}{l}0.00 \\
51\end{array}$ & $\begin{array}{l}0.05 \\
94\end{array}$ & $\begin{array}{l}0.00 \\
17\end{array}$ & 522 & $\begin{array}{l}14 \\
.5\end{array}$ & 510 & 15 & 581 & 32 & 510 & yes \\
\hline ODP_104 & 225 & 131.2 & 1.64 & 0.866 & $\begin{array}{l}0.0 \\
64\end{array}$ & 0.0798 & $\begin{array}{l}0.00 \\
46\end{array}$ & $\begin{array}{l}0.07 \\
83\end{array}$ & $\begin{array}{l}0.00 \\
22\end{array}$ & 631 & 17 & 495 & 14 & 1146 & $\begin{array}{l}28 \\
.5\end{array}$ & 495 & yes \\
\hline ODP_105 & $\begin{array}{l}111 \\
.2 \\
\end{array}$ & 76 & 1.45 & 1.45 & $\begin{array}{l}0.1 \\
3 \\
\end{array}$ & 0.0873 & $\begin{array}{l}0.00 \\
53 \\
\end{array}$ & $\begin{array}{l}0.12 \\
15 \\
\end{array}$ & $\begin{array}{l}0.00 \\
62 \\
\end{array}$ & 901 & 26 & 539 & $\begin{array}{l}15 \\
.5 \\
\end{array}$ & 1984 & $\begin{array}{l}43 \\
.5 \\
\end{array}$ & 539 & yes \\
\hline ODP_107 & 206 & 225 & 0.856 & 1.064 & $\begin{array}{l}0.0 \\
78\end{array}$ & 0.0831 & $\begin{array}{l}0.00 \\
48\end{array}$ & $\begin{array}{l}0.09 \\
22\end{array}$ & $\begin{array}{l}0.00 \\
32\end{array}$ & 733 & 19 & 514 & $\begin{array}{l}14 \\
.5\end{array}$ & 1458 & $\begin{array}{l}32 \\
.5\end{array}$ & 514 & yes \\
\hline ODP_108 & 268 & 145.4 & 1.77 & 1.19 & $\begin{array}{l}0.0 \\
84 \\
\end{array}$ & 0.0829 & $\begin{array}{l}0.00 \\
47 \\
\end{array}$ & $\begin{array}{l}0.10 \\
71 \\
\end{array}$ & $\begin{array}{l}0.00 \\
34 \\
\end{array}$ & 801 & 18 & 513 & 14 & 1740 & $\begin{array}{l}29 \\
.5 \\
\end{array}$ & 513 & yes \\
\hline ODP_109 & $\begin{array}{l}178 \\
.1 \\
\end{array}$ & 122.3 & 1.417 & 1.036 & $\begin{array}{l}0.0 \\
75 \\
\end{array}$ & 0.0809 & $\begin{array}{l}0.00 \\
51 \\
\end{array}$ & $\begin{array}{l}0.09 \\
25 \\
\end{array}$ & $\begin{array}{l}0.00 \\
32 \\
\end{array}$ & 719 & $\begin{array}{l}18 \\
.5 \\
\end{array}$ & 501 & 15 & 1465 & 33 & 501 & yes \\
\hline \multicolumn{18}{|l|}{778} \\
\hline Analysis & $\begin{array}{l}\text { U } \\
\text { pp } \\
\mathrm{m}\end{array}$ & $\begin{array}{l}\text { Th } \\
\text { ppm }\end{array}$ & $\mathrm{U} / \mathrm{Th}$ & $\begin{array}{l}207 \mathrm{~Pb} / 2 \\
35 \mathrm{U}\end{array}$ & $\begin{array}{l}+/- \\
2 \sigma\end{array}$ & $\begin{array}{l}206 \mathrm{~Pb} / 2 \\
38 \mathrm{U}\end{array}$ & $\begin{array}{l}+/- \\
2 \sigma\end{array}$ & $\begin{array}{l}\text { 207P } \\
\text { b/ } \\
\text { 206P } \\
\text { b }\end{array}$ & $\begin{array}{l}+/- \\
2 \sigma\end{array}$ & $\begin{array}{l}207 P \\
\text { b/ } \\
235 \\
\text { U } \\
\text { (Ma) }\end{array}$ & $\begin{array}{l}+1 \\
- \\
\sigma \\
M \\
a\end{array}$ & $\begin{array}{l}206 \mathrm{~Pb} / \\
235 \mathrm{U} \\
\text { (Ma) }\end{array}$ & $\begin{array}{l}+1 \\
- \\
\sigma \\
M \\
a\end{array}$ & $\begin{array}{l}207 \mathrm{P} \\
\text { b/ } \\
206 \mathrm{P} \\
\mathrm{b} \\
\text { (Ma) }\end{array}$ & $\begin{array}{l}+1 \\
- \\
\sigma \\
M \\
a\end{array}$ & $\begin{array}{l}\text { Bes } \\
\mathrm{t} \\
\mathrm{Ag} \\
\mathrm{e} \\
\mathrm{Ma}\end{array}$ & $\begin{array}{l}\text { Use } \\
\text { d? }\end{array}$ \\
\hline X778_2 & $\begin{array}{l}141 \\
.4\end{array}$ & 95.8 & 1.492 & 0.805 & $\begin{array}{l}0.0 \\
51\end{array}$ & 0.0893 & $\begin{array}{l}0.00 \\
29\end{array}$ & $\begin{array}{l}0.06 \\
52\end{array}$ & $\begin{array}{l}0.00 \\
35\end{array}$ & 596 & 14 & 551 & $\begin{array}{l}8 . \\
5\end{array}$ & 750 & 55 & 551 & yes \\
\hline X778_3 & 157 & 46.2 & 3.4 & 0.712 & $\begin{array}{l}0.0 \\
31\end{array}$ & 0.0897 & $\begin{array}{l}0.00 \\
3\end{array}$ & $\begin{array}{l}0.05 \\
83\end{array}$ & $\begin{array}{l}0.00 \\
16\end{array}$ & 551 & 7 & 554 & 9 & 530 & $\begin{array}{l}31 \\
.5\end{array}$ & 554 & yes \\
\hline X778_4 & 69 & 33.9 & 1.79 & 0.819 & $\begin{array}{l}0.0 \\
74 \\
\end{array}$ & 0.098 & $\begin{array}{l}0.00 \\
44 \\
\end{array}$ & $\begin{array}{l}0.06 \\
25 \\
\end{array}$ & $\begin{array}{l}0.00 \\
39 \\
\end{array}$ & 611 & $\begin{array}{l}18 \\
.5 \\
\end{array}$ & 603 & 13 & 650 & 65 & 603 & yes \\
\hline X778_5 & $\begin{array}{l}58 . \\
9\end{array}$ & 152.2 & 0.389 & 0.88 & 0.1 & 0.0872 & $\begin{array}{l}0.00 \\
27 \\
\end{array}$ & $\begin{array}{l}0.07 \\
15 \\
\end{array}$ & $\begin{array}{l}0.00 \\
73 \\
\end{array}$ & 629 & 25 & 539 & 8 & 890 & $\begin{array}{l}10 \\
0 \\
\end{array}$ & 539 & yes \\
\hline X778_6 & 90 & 30.56 & 2.96 & 0.737 & $\begin{array}{l}0.0 \\
4 \\
\end{array}$ & 0.0868 & $\begin{array}{l}0.00 \\
31 \\
\end{array}$ & $\begin{array}{l}0.06 \\
25 \\
\end{array}$ & $\begin{array}{l}0.00 \\
32 \\
\end{array}$ & 565 & $\begin{array}{l}12 \\
.5 \\
\end{array}$ & 536 & $\begin{array}{l}9 . \\
5 \\
\end{array}$ & 660 & 50 & 536 & yes \\
\hline X778_8 & 199 & 25.3 & 7.69 & 2.01 & $\begin{array}{l}0.1 \\
1 \\
\end{array}$ & 0.1491 & $\begin{array}{l}0.00 \\
58 \\
\end{array}$ & $\begin{array}{l}0.09 \\
96 \\
\end{array}$ & $\begin{array}{l}0.00 \\
29 \\
\end{array}$ & 1126 & $\begin{array}{l}18 \\
.5 \\
\end{array}$ & 896 & 16 & 1619 & $\begin{array}{l}26 \\
.5 \\
\end{array}$ & 896 & no \\
\hline X778_9 & $\begin{array}{l}60 . \\
8\end{array}$ & 73.8 & 0.811 & 0.971 & $\begin{array}{l}0.0 \\
71\end{array}$ & 0.0926 & $\begin{array}{l}0.00 \\
31\end{array}$ & $\begin{array}{l}0.07 \\
45\end{array}$ & $\begin{array}{l}0.00 \\
52\end{array}$ & 684 & $\begin{array}{l}17 \\
.5\end{array}$ & 570 & 9 & 1060 & 70 & 570 & yes \\
\hline X778_10 & 271 & 109 & 2.545 & 0.682 & $\begin{array}{l}0.0 \\
23\end{array}$ & 0.0822 & $\begin{array}{l}0.00 \\
28\end{array}$ & $\begin{array}{l}0.05 \\
92\end{array}$ & $\begin{array}{l}0.00 \\
14\end{array}$ & 527 & 7 & 509 & $\begin{array}{l}8 . \\
5\end{array}$ & 575 & $\begin{array}{l}25 \\
.5\end{array}$ & 509 & yes \\
\hline X778_11 & $\begin{array}{l}262 \\
.8\end{array}$ & 73.4 & 3.59 & 0.676 & $\begin{array}{l}0.0 \\
26\end{array}$ & 0.0641 & $\begin{array}{l}0.00 \\
22\end{array}$ & $\begin{array}{l}0.07 \\
65\end{array}$ & $\begin{array}{l}0.00 \\
25\end{array}$ & 524 & $\begin{array}{l}7 . \\
5\end{array}$ & 400.6 & 7 & 1098 & 32 & $\begin{array}{l}400 \\
.6\end{array}$ & yes \\
\hline X778_12 & $\begin{array}{l}\text { 62. } \\
6\end{array}$ & 42 & 1.529 & 0.738 & $\begin{array}{l}0.0 \\
36\end{array}$ & 0.0888 & $\begin{array}{l}0.00 \\
3\end{array}$ & $\begin{array}{l}0.06 \\
18\end{array}$ & $\begin{array}{l}0.00 \\
25\end{array}$ & 559 & $\begin{array}{l}10 \\
.5\end{array}$ & 548 & $\begin{array}{l}8 . \\
5\end{array}$ & 659 & $\begin{array}{l}44 \\
.5\end{array}$ & 548 & yes \\
\hline X778_13 & $\begin{array}{l}125 \\
1\end{array}$ & 746 & 1.62 & 0.536 & $\begin{array}{l}0.0 \\
3\end{array}$ & 0.052 & $\begin{array}{l}0.00 \\
27\end{array}$ & $\begin{array}{l}0.07 \\
7\end{array}$ & $\begin{array}{l}0.00 \\
28\end{array}$ & 435 & $\begin{array}{l}9 . \\
5\end{array}$ & 326 & $\begin{array}{l}8 . \\
5\end{array}$ & 1115 & 37 & 326 & yes \\
\hline X778_14 & 117 & 21.7 & 4.93 & 0.849 & $\begin{array}{l}0.0 \\
49 \\
\end{array}$ & 0.0941 & $\begin{array}{l}0.00 \\
43 \\
\end{array}$ & $\begin{array}{l}0.06 \\
47 \\
\end{array}$ & $\begin{array}{l}0.00 \\
26 \\
\end{array}$ & 621 & 13 & 579 & $\begin{array}{l}12 \\
.5 \\
\end{array}$ & 746 & 43 & 579 & yes \\
\hline X778_15 & 667 & 159 & 5.8 & 0.688 & $\begin{array}{l}0.0 \\
35\end{array}$ & 0.0777 & $\begin{array}{l}0.00 \\
42\end{array}$ & $\begin{array}{l}0.06 \\
61\end{array}$ & $\begin{array}{l}0.00 \\
4\end{array}$ & 537 & $\begin{array}{l}12 \\
.5\end{array}$ & 482 & $\begin{array}{l}12 \\
.5\end{array}$ & 780 & 55 & 482 & yes \\
\hline X778_16 & $\begin{array}{l}137 \\
.6 \\
\end{array}$ & 74.2 & 1.946 & 0.715 & $\begin{array}{l}0.0 \\
26 \\
\end{array}$ & 0.0845 & $\begin{array}{l}0.00 \\
25 \\
\end{array}$ & $\begin{array}{l}0.06 \\
14 \\
\end{array}$ & $\begin{array}{l}0.00 \\
2 \\
\end{array}$ & 547 & $\begin{array}{l}7 . \\
5\end{array}$ & 522.7 & $\begin{array}{l}7 . \\
5 \\
\end{array}$ & 642 & $\begin{array}{l}33 \\
.5 \\
\end{array}$ & $\begin{array}{l}522 \\
.7 \\
\end{array}$ & yes \\
\hline X778_17 & $\begin{array}{l}165 \\
.4\end{array}$ & 154.2 & 1.088 & 0.742 & $\begin{array}{l}0.0 \\
32\end{array}$ & 0.0902 & $\begin{array}{l}0.00 \\
32\end{array}$ & $\begin{array}{l}0.05 \\
93\end{array}$ & $\begin{array}{l}0.00 \\
23\end{array}$ & 562 & $\begin{array}{l}9 . \\
5\end{array}$ & 557 & $\begin{array}{l}9 . \\
5\end{array}$ & 598 & $\begin{array}{l}42 \\
.5\end{array}$ & 557 & yes \\
\hline X778_18 & $\begin{array}{l}20 . \\
89\end{array}$ & 24.65 & 0.852 & 1.15 & 0.2 & 0.0884 & $\begin{array}{l}0.00 \\
37\end{array}$ & $\begin{array}{l}0.09 \\
3\end{array}$ & $\begin{array}{l}0.01 \\
5\end{array}$ & 727 & $\begin{array}{l}37 \\
.5\end{array}$ & 546 & 11 & 1240 & $\begin{array}{l}13 \\
5\end{array}$ & 546 & yes \\
\hline X778_19 & $\begin{array}{l}320 \\
.7\end{array}$ & 186.4 & 1.727 & 0.928 & $\begin{array}{l}0.0 \\
45\end{array}$ & 0.0929 & $\begin{array}{l}0.00 \\
28\end{array}$ & $\begin{array}{l}0.07 \\
28\end{array}$ & $\begin{array}{l}0.00 \\
34\end{array}$ & 665 & $\begin{array}{l}11 \\
.5\end{array}$ & 572.5 & 8 & 984 & $\begin{array}{l}45 \\
.5\end{array}$ & $\begin{array}{l}572 \\
.5\end{array}$ & yes \\
\hline X778_20 & 540 & 404 & 1.41 & 2.49 & $\begin{array}{l}0.2 \\
2\end{array}$ & 0.1353 & $\begin{array}{l}0.00 \\
58\end{array}$ & $\begin{array}{l}0.13 \\
3\end{array}$ & $\begin{array}{l}0.01 \\
2\end{array}$ & 1259 & 32 & 818 & $\begin{array}{l}16 \\
.5\end{array}$ & 2100 & 75 & 818 & no \\
\hline
\end{tabular}




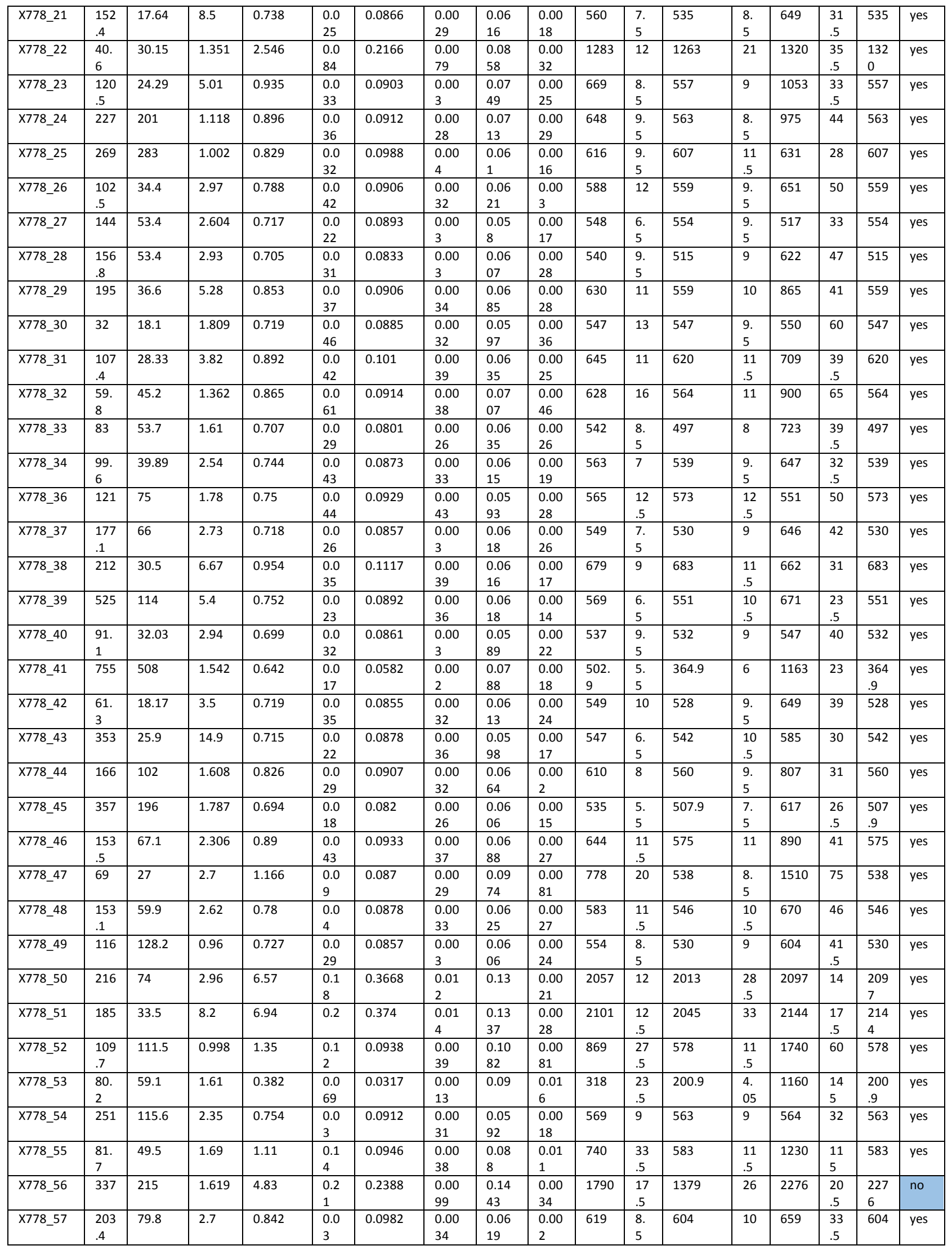




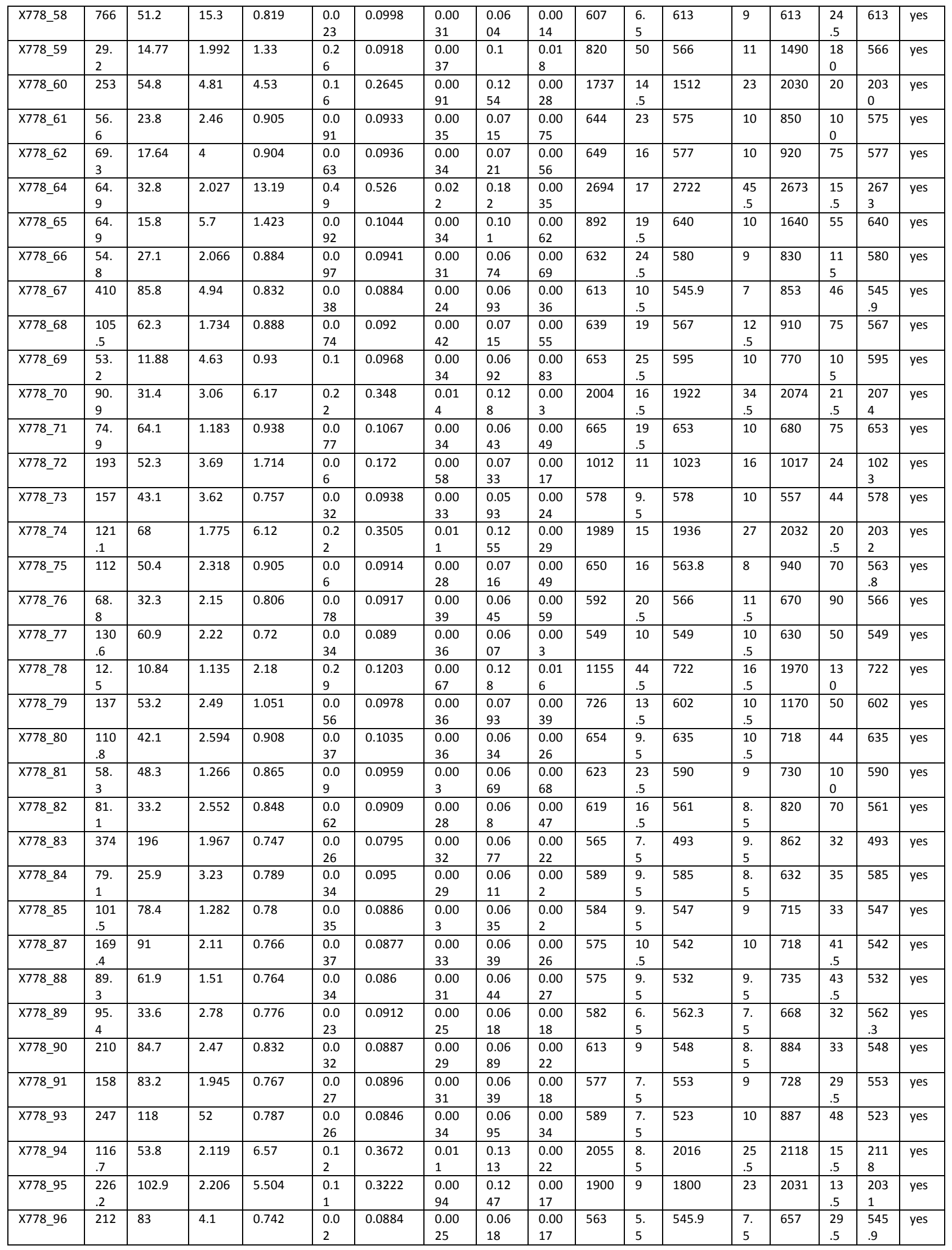




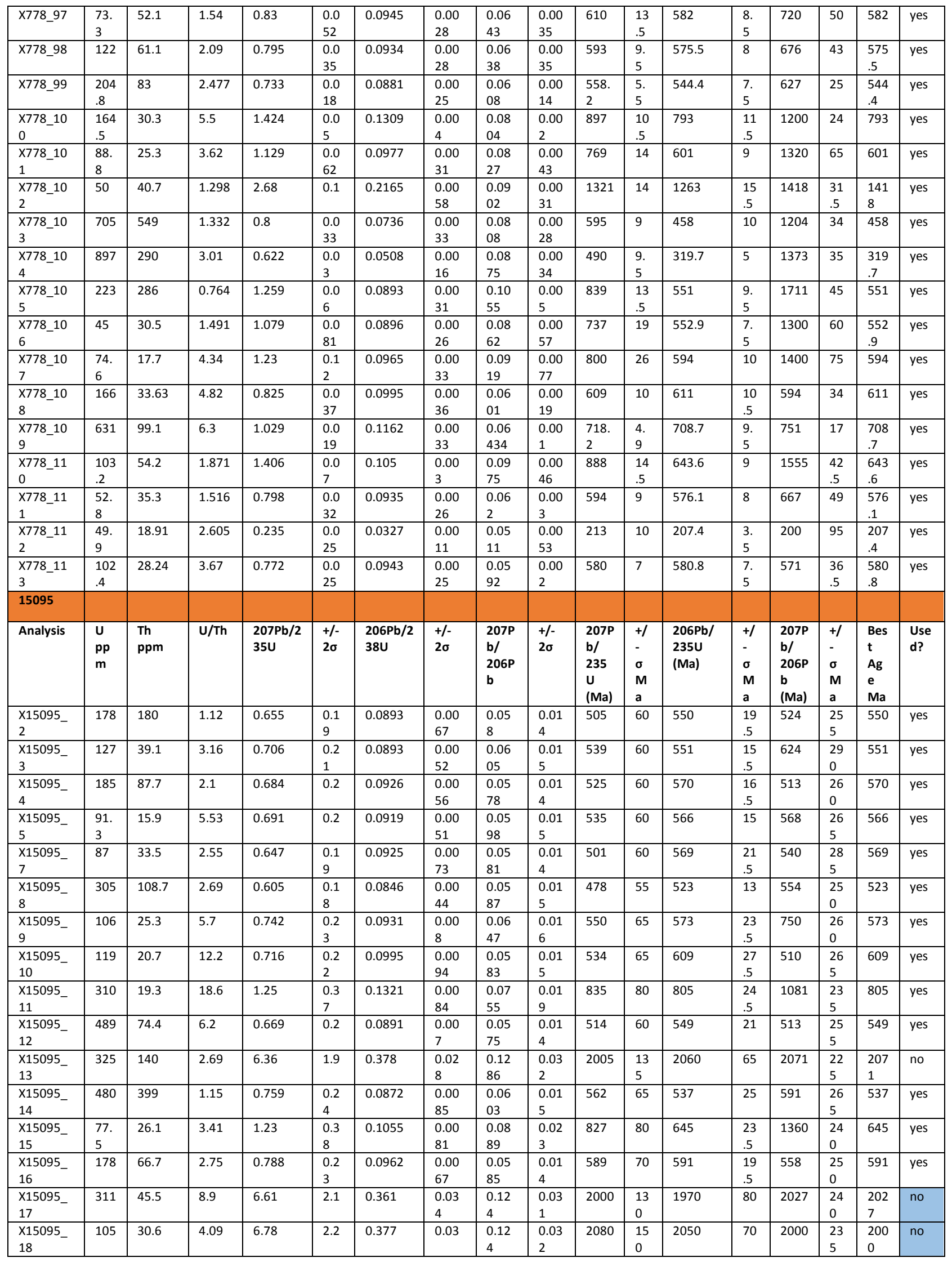




\begin{tabular}{|c|c|c|c|c|c|c|c|c|c|c|c|c|c|c|c|c|c|}
\hline $\begin{array}{l}\text { X15095_ } \\
19\end{array}$ & 263 & 108 & 2.34 & 0.917 & $\begin{array}{l}0.2 \\
8 \\
\end{array}$ & 0.11 & $\begin{array}{l}0.00 \\
81 \\
\end{array}$ & $\begin{array}{l}0.05 \\
84 \\
\end{array}$ & $\begin{array}{l}0.01 \\
5 \\
\end{array}$ & 657 & 70 & 672 & $\begin{array}{l}23 \\
.5 \\
\end{array}$ & 533 & $\begin{array}{l}26 \\
5 \\
\end{array}$ & 672 & yes \\
\hline $\begin{array}{l}\text { X15095_ } \\
20\end{array}$ & 139 & 14.3 & 8.7 & 0.829 & $\begin{array}{l}0.2 \\
5 \\
\end{array}$ & 0.1 & $\begin{array}{l}0.00 \\
7 \\
\end{array}$ & $\begin{array}{l}0.05 \\
87 \\
\end{array}$ & $\begin{array}{l}0.01 \\
5 \\
\end{array}$ & 603 & 70 & 613 & $\begin{array}{l}20 \\
.5 \\
\end{array}$ & 520 & $\begin{array}{l}26 \\
5 \\
\end{array}$ & 613 & yes \\
\hline $\begin{array}{l}\text { X15095_- } \\
21\end{array}$ & 611 & 59.8 & 10.5 & 6.9 & 2.2 & 0.356 & $\begin{array}{l}0.04 \\
3\end{array}$ & $\begin{array}{l}0.13 \\
46 \\
\end{array}$ & $\begin{array}{l}0.03 \\
4 \\
\end{array}$ & 2040 & $\begin{array}{l}14 \\
5 \\
\end{array}$ & 1940 & $\begin{array}{l}10 \\
0 \\
\end{array}$ & 2140 & $\begin{array}{l}21 \\
5 \\
\end{array}$ & $\begin{array}{l}214 \\
0 \\
\end{array}$ & yes \\
\hline $\begin{array}{l}\text { X15095_ } \\
22\end{array}$ & $\begin{array}{l}71 . \\
2\end{array}$ & 26.8 & 2.61 & 0.919 & $\begin{array}{l}0.2 \\
7 \\
\end{array}$ & 0.1096 & $\begin{array}{l}0.00 \\
7\end{array}$ & $\begin{array}{l}0.06 \\
05\end{array}$ & $\begin{array}{l}0.01 \\
5\end{array}$ & 655 & 70 & 670 & $\begin{array}{l}20 \\
.5\end{array}$ & 616 & $\begin{array}{l}28 \\
0\end{array}$ & 670 & yes \\
\hline $\begin{array}{l}\text { X15095_ } \\
23\end{array}$ & 112 & 34.2 & 3.55 & 0.9 & $\begin{array}{l}0.2 \\
8 \\
\end{array}$ & 0.1051 & $\begin{array}{l}0.00 \\
83 \\
\end{array}$ & $\begin{array}{l}0.05 \\
95\end{array}$ & $\begin{array}{l}0.01 \\
5\end{array}$ & 635 & 75 & 642 & 24 & 560 & $\begin{array}{l}27 \\
5\end{array}$ & 642 & yes \\
\hline $\begin{array}{l}\text { X15095_ } \\
24\end{array}$ & 125 & 48.6 & 2.5 & 0.811 & $\begin{array}{l}0.2 \\
4\end{array}$ & 0.0993 & $\begin{array}{l}0.00 \\
62\end{array}$ & $\begin{array}{l}0.06 \\
03\end{array}$ & $\begin{array}{l}0.01 \\
5\end{array}$ & 598 & 65 & 610 & $\begin{array}{l}18 \\
.5\end{array}$ & 595 & $\begin{array}{l}26 \\
5\end{array}$ & 610 & yes \\
\hline $\begin{array}{l}\text { X15095_ } \\
25\end{array}$ & 195 & 63 & 3.31 & 0.812 & $\begin{array}{l}0.2 \\
5\end{array}$ & 0.0944 & $\begin{array}{l}0.00 \\
85\end{array}$ & 0.06 & $\begin{array}{l}0.01 \\
5\end{array}$ & 598 & 70 & 580 & 25 & 570 & $\begin{array}{l}27 \\
0\end{array}$ & 580 & yes \\
\hline $\begin{array}{l}\text { X15095_ } \\
26\end{array}$ & 224 & 28.9 & 7.6 & 0.868 & $\begin{array}{l}0.2 \\
6\end{array}$ & 0.1039 & $\begin{array}{l}0.00 \\
78\end{array}$ & $\begin{array}{l}0.05 \\
94\end{array}$ & $\begin{array}{l}0.01 \\
5\end{array}$ & 625 & 70 & 636 & $\begin{array}{l}22 \\
.5\end{array}$ & 574 & $\begin{array}{l}27 \\
5\end{array}$ & 636 & yes \\
\hline $\begin{array}{l}\text { X15095_ } \\
27\end{array}$ & 180 & 98 & 1.76 & 0.797 & $\begin{array}{l}0.2 \\
3\end{array}$ & 0.0944 & $\begin{array}{l}0.00 \\
52\end{array}$ & $\begin{array}{l}0.06 \\
2\end{array}$ & $\begin{array}{l}0.01 \\
5\end{array}$ & 596 & 65 & 581 & 15 & 669 & $\begin{array}{l}27 \\
0\end{array}$ & 581 & yes \\
\hline $\begin{array}{l}\text { X15095_ } \\
29\end{array}$ & 216 & 50.6 & 3.86 & 0.821 & $\begin{array}{l}0.2 \\
4 \\
\end{array}$ & 0.097 & $\begin{array}{l}0.00 \\
59 \\
\end{array}$ & $\begin{array}{l}0.06 \\
12 \\
\end{array}$ & $\begin{array}{l}0.01 \\
5 \\
\end{array}$ & 608 & 65 & 603 & $\begin{array}{l}18 \\
.5 \\
\end{array}$ & 623 & $\begin{array}{l}25 \\
5 \\
\end{array}$ & 603 & yes \\
\hline $\begin{array}{l}\text { X15095_ } \\
30\end{array}$ & $\begin{array}{l}87 . \\
7\end{array}$ & 104.2 & 0.82 & 2.11 & $\begin{array}{l}0.6 \\
3\end{array}$ & 0.1918 & $\begin{array}{l}0.01 \\
1\end{array}$ & $\begin{array}{l}0.07 \\
77\end{array}$ & $\begin{array}{l}0.01 \\
9\end{array}$ & 1136 & $\begin{array}{l}10 \\
0\end{array}$ & 1129 & $\begin{array}{l}30 \\
.5\end{array}$ & 1159 & $\begin{array}{l}25 \\
0\end{array}$ & $\begin{array}{l}112 \\
9\end{array}$ & yes \\
\hline $\begin{array}{l}\text { X15095_ } \\
31\end{array}$ & 209 & 12.7 & 16.3 & 0.755 & $\begin{array}{l}0.2 \\
3 \\
\end{array}$ & 0.094 & $\begin{array}{l}0.00 \\
66 \\
\end{array}$ & $\begin{array}{l}0.05 \\
76 \\
\end{array}$ & $\begin{array}{l}0.01 \\
4 \\
\end{array}$ & 571 & 60 & 578 & $\begin{array}{l}19 \\
.5 \\
\end{array}$ & 495 & $\begin{array}{l}27 \\
5 \\
\end{array}$ & 578 & yes \\
\hline $\begin{array}{l}\text { X15095_ } \\
34\end{array}$ & $\begin{array}{l}76 . \\
2\end{array}$ & 40.2 & 1.96 & 0.863 & $\begin{array}{l}0.2 \\
6\end{array}$ & 0.0981 & $\begin{array}{l}0.00 \\
68\end{array}$ & $\begin{array}{l}0.06 \\
44\end{array}$ & $\begin{array}{l}0.01 \\
6\end{array}$ & 645 & 65 & 602 & 20 & 760 & $\begin{array}{l}28 \\
0\end{array}$ & 602 & yes \\
\hline $\begin{array}{l}\text { X15095_ } \\
35\end{array}$ & 346 & 44.7 & 8.9 & 0.94 & $\begin{array}{l}0.3 \\
1 \\
\end{array}$ & 0.115 & $\begin{array}{l}0.01 \\
6 \\
\end{array}$ & $\begin{array}{l}0.05 \\
75 \\
\end{array}$ & $\begin{array}{l}0.01 \\
4 \\
\end{array}$ & 642 & 75 & 674 & 40 & 500 & $\begin{array}{l}28 \\
0 \\
\end{array}$ & 674 & no \\
\hline $\begin{array}{l}\text { X15095_ } \\
36\end{array}$ & 194 & 39.8 & 4.67 & 7.79 & 2.4 & 0.433 & $\begin{array}{l}0.03 \\
7\end{array}$ & $\begin{array}{l}0.12 \\
78\end{array}$ & $\begin{array}{l}0.03 \\
2\end{array}$ & 2160 & $\begin{array}{l}13 \\
5\end{array}$ & 2300 & 80 & 2062 & $\begin{array}{l}21 \\
5\end{array}$ & $\begin{array}{l}206 \\
2\end{array}$ & no \\
\hline $\begin{array}{l}\mathrm{X} 15095 \\
38\end{array}$ & 250 & 167 & 1.83 & 3.44 & 1.1 & 0.266 & $\begin{array}{l}0.02 \\
3 \\
\end{array}$ & $\begin{array}{l}0.09 \\
19 \\
\end{array}$ & $\begin{array}{l}0.02 \\
3 \\
\end{array}$ & 1501 & $\begin{array}{l}11 \\
5 \\
\end{array}$ & 1520 & 60 & 1469 & $\begin{array}{l}22 \\
5 \\
\end{array}$ & $\begin{array}{l}146 \\
9 \\
\end{array}$ & no \\
\hline $\begin{array}{l}\text { X15095_ } \\
39\end{array}$ & $\begin{array}{l}48 . \\
6\end{array}$ & 32.8 & 1.61 & 0.926 & $\begin{array}{l}0.2 \\
8\end{array}$ & 0.1058 & $\begin{array}{l}0.00 \\
76\end{array}$ & $\begin{array}{l}0.06 \\
36\end{array}$ & $\begin{array}{l}0.01 \\
6\end{array}$ & 656 & 70 & 647 & 22 & 680 & $\begin{array}{l}25 \\
0\end{array}$ & 647 & yes \\
\hline $\begin{array}{l}\text { X15095_ } \\
41\end{array}$ & $\begin{array}{l}34 . \\
1\end{array}$ & 15.5 & 2.21 & 0.93 & $\begin{array}{l}0.3 \\
2\end{array}$ & 0.099 & $\begin{array}{l}0.01 \\
2\end{array}$ & $\begin{array}{l}0.06 \\
38\end{array}$ & $\begin{array}{l}0.01 \\
7\end{array}$ & 654 & 80 & 605 & 34 & 650 & $\begin{array}{l}26 \\
5\end{array}$ & 605 & no \\
\hline $\begin{array}{l}\text { X15095_ } \\
42\end{array}$ & 126 & 24 & 5.4 & 0.81 & $\begin{array}{l}0.2 \\
5 \\
\end{array}$ & 0.103 & $\begin{array}{l}0.00 \\
85 \\
\end{array}$ & $\begin{array}{l}0.05 \\
64 \\
\end{array}$ & $\begin{array}{l}0.01 \\
4 \\
\end{array}$ & 597 & 70 & 630 & $\begin{array}{l}24 \\
.5 \\
\end{array}$ & 450 & $\begin{array}{l}28 \\
5 \\
\end{array}$ & 630 & yes \\
\hline $\begin{array}{l}\text { X15095_ } \\
43\end{array}$ & 141 & 50.5 & 2.62 & 0.79 & $\begin{array}{l}0.2 \\
4\end{array}$ & 0.0991 & $\begin{array}{l}0.00 \\
73\end{array}$ & $\begin{array}{l}0.05 \\
82\end{array}$ & $\begin{array}{l}0.01 \\
4\end{array}$ & 590 & 65 & 608 & $\begin{array}{l}21 \\
.5\end{array}$ & 547 & $\begin{array}{l}25 \\
0\end{array}$ & 608 & yes \\
\hline $\begin{array}{l}\text { X15095_ } \\
44\end{array}$ & 157 & 50.1 & 3.8 & 0.76 & $\begin{array}{l}0.2 \\
4 \\
\end{array}$ & 0.097 & $\begin{array}{l}0.01 \\
1 \\
\end{array}$ & $\begin{array}{l}0.05 \\
64 \\
\end{array}$ & $\begin{array}{l}0.01 \\
4 \\
\end{array}$ & 568 & 70 & 595 & $\begin{array}{l}30 \\
.5 \\
\end{array}$ & 460 & $\begin{array}{l}25 \\
5 \\
\end{array}$ & 595 & no \\
\hline $\begin{array}{l}\text { X15095_ } \\
45\end{array}$ & 161 & 65 & 4.2 & 0.8 & $\begin{array}{l}0.2 \\
6\end{array}$ & 0.0987 & $\begin{array}{l}0.00 \\
99\end{array}$ & $\begin{array}{l}0.05 \\
85\end{array}$ & $\begin{array}{l}0.01 \\
5\end{array}$ & 578 & 70 & 604 & 29 & 540 & $\begin{array}{l}28 \\
5\end{array}$ & 604 & yes \\
\hline $\begin{array}{l}\text { X15095_ } \\
46\end{array}$ & 146 & 147 & 1.24 & 0.84 & $\begin{array}{l}0.2 \\
8 \\
\end{array}$ & 0.0945 & $\begin{array}{l}0.00 \\
87 \\
\end{array}$ & $\begin{array}{l}0.06 \\
19 \\
\end{array}$ & $\begin{array}{l}0.01 \\
6\end{array}$ & 580 & 60 & 580 & $\begin{array}{l}25 \\
.5 \\
\end{array}$ & 600 & $\begin{array}{l}25 \\
0\end{array}$ & 580 & yes \\
\hline $\begin{array}{l}\text { X15095_ } \\
47\end{array}$ & 386 & 51.7 & 10.1 & 6.42 & 2.1 & 0.335 & $\begin{array}{l}0.03 \\
7\end{array}$ & $\begin{array}{l}0.13 \\
5\end{array}$ & $\begin{array}{l}0.03 \\
4\end{array}$ & 1950 & $\begin{array}{l}14 \\
0\end{array}$ & 1840 & 85 & 2180 & $\begin{array}{l}22 \\
0\end{array}$ & $\begin{array}{l}218 \\
0\end{array}$ & no \\
\hline $\begin{array}{l}\text { X15095_- } \\
48\end{array}$ & $\begin{array}{l}69 . \\
4\end{array}$ & 29.4 & 2.25 & 1.218 & $\begin{array}{l}0.3 \\
6\end{array}$ & 0.129 & $\begin{array}{l}0.00 \\
69\end{array}$ & $\begin{array}{l}0.06 \\
81 \\
\end{array}$ & $\begin{array}{l}0.01 \\
7 \\
\end{array}$ & 801 & 80 & 781 & $\begin{array}{l}19 \\
.5\end{array}$ & 840 & $\begin{array}{l}25 \\
0\end{array}$ & 781 & yes \\
\hline $\begin{array}{l}\text { X15095_ } \\
49\end{array}$ & 471 & 142 & 3.9 & 0.753 & $\begin{array}{l}0.2 \\
3\end{array}$ & 0.0901 & $\begin{array}{l}0.00 \\
63\end{array}$ & 0.06 & $\begin{array}{l}0.01 \\
5\end{array}$ & 561 & 65 & 555 & $\begin{array}{l}18 \\
.5\end{array}$ & 610 & $\begin{array}{l}25 \\
5\end{array}$ & 555 & yes \\
\hline $\begin{array}{l}\text { X15095_ } \\
50\end{array}$ & $\begin{array}{l}95 . \\
5\end{array}$ & 57.5 & 1.67 & 0.781 & $\begin{array}{l}0.2 \\
3 \\
\end{array}$ & 0.0984 & $\begin{array}{l}0.00 \\
66\end{array}$ & $\begin{array}{l}0.06 \\
21 \\
\end{array}$ & $\begin{array}{l}0.01 \\
6\end{array}$ & 577 & 65 & 604 & $\begin{array}{l}19 \\
.5\end{array}$ & 640 & $\begin{array}{l}25 \\
0\end{array}$ & 604 & yes \\
\hline $\begin{array}{l}\text { X15095_ } \\
51\end{array}$ & 266 & 31.8 & 8.8 & 0.679 & $\begin{array}{l}0.2 \\
1 \\
\end{array}$ & 0.089 & $\begin{array}{l}0.00 \\
93 \\
\end{array}$ & $\begin{array}{l}0.05 \\
73 \\
\end{array}$ & $\begin{array}{l}0.01 \\
4 \\
\end{array}$ & 521 & 65 & 548 & 27 & 508 & $\begin{array}{l}26 \\
5 \\
\end{array}$ & 548 & yes \\
\hline $\begin{array}{l}\text { X15095_ } \\
52\end{array}$ & $\begin{array}{l}38 . \\
8\end{array}$ & 13.7 & 2.69 & 0.649 & $\begin{array}{l}0.1 \\
9 \\
\end{array}$ & 0.0777 & $\begin{array}{l}0.00 \\
41 \\
\end{array}$ & $\begin{array}{l}0.06 \\
5 \\
\end{array}$ & $\begin{array}{l}0.01 \\
6 \\
\end{array}$ & 504 & 60 & 482 & 12 & 775 & $\begin{array}{l}26 \\
5 \\
\end{array}$ & 482 & yes \\
\hline $\begin{array}{l}\text { X15095_ } \\
53\end{array}$ & $\begin{array}{l}72 . \\
9\end{array}$ & 53.7 & 1.38 & 0.591 & $\begin{array}{l}0.1 \\
7\end{array}$ & 0.0839 & $\begin{array}{l}0.00 \\
48\end{array}$ & $\begin{array}{l}0.05 \\
63\end{array}$ & $\begin{array}{l}0.01 \\
4\end{array}$ & 472 & 55 & 519 & 14 & 445 & $\begin{array}{l}27 \\
0\end{array}$ & 519 & yes \\
\hline $\begin{array}{l}X 15095 \_ \\
54\end{array}$ & $\begin{array}{l}14 . \\
5\end{array}$ & 8.4 & 1.64 & 1.09 & $\begin{array}{l}0.5 \\
2 \\
\end{array}$ & 0.0855 & $\begin{array}{l}0.00 \\
8 \\
\end{array}$ & $\begin{array}{l}0.08 \\
5 \\
\end{array}$ & $\begin{array}{l}0.02 \\
9 \\
\end{array}$ & 537 & 50 & 527 & $\begin{array}{l}23 \\
.5 \\
\end{array}$ & 930 & $\begin{array}{l}22 \\
0 \\
\end{array}$ & 527 & yes \\
\hline $\begin{array}{l}\text { X15095_ } \\
55\end{array}$ & 148 & 67.9 & 1.95 & 0.617 & $\begin{array}{l}0.1 \\
8\end{array}$ & 0.0863 & $\begin{array}{l}0.00 \\
55\end{array}$ & $\begin{array}{l}0.05 \\
69\end{array}$ & $\begin{array}{l}0.01 \\
4\end{array}$ & 483 & 55 & 533 & $\begin{array}{l}16 \\
.5\end{array}$ & 499 & $\begin{array}{l}27 \\
5\end{array}$ & 533 & yes \\
\hline $\begin{array}{l}\text { X15095_ } \\
56\end{array}$ & $\begin{array}{l}72 . \\
8\end{array}$ & 39 & 2.13 & 0.59 & $\begin{array}{l}0.1 \\
7\end{array}$ & 0.079 & $\begin{array}{l}0.00 \\
53\end{array}$ & $\begin{array}{l}0.06 \\
15\end{array}$ & $\begin{array}{l}0.01 \\
5\end{array}$ & 466 & 55 & 490 & $\begin{array}{l}15 \\
.5\end{array}$ & 586 & $\begin{array}{l}19 \\
5\end{array}$ & 490 & yes \\
\hline $\begin{array}{l}\text { X15095_ } \\
57\end{array}$ & $\begin{array}{l}121 \\
.4\end{array}$ & 26.4 & 4.55 & 0.586 & $\begin{array}{l}0.1 \\
7\end{array}$ & 0.0855 & $\begin{array}{l}0.00 \\
54\end{array}$ & $\begin{array}{l}0.05 \\
6\end{array}$ & $\begin{array}{l}0.01 \\
4\end{array}$ & 469 & 55 & 528 & 16 & 453 & $\begin{array}{l}28 \\
5\end{array}$ & 528 & yes \\
\hline
\end{tabular}




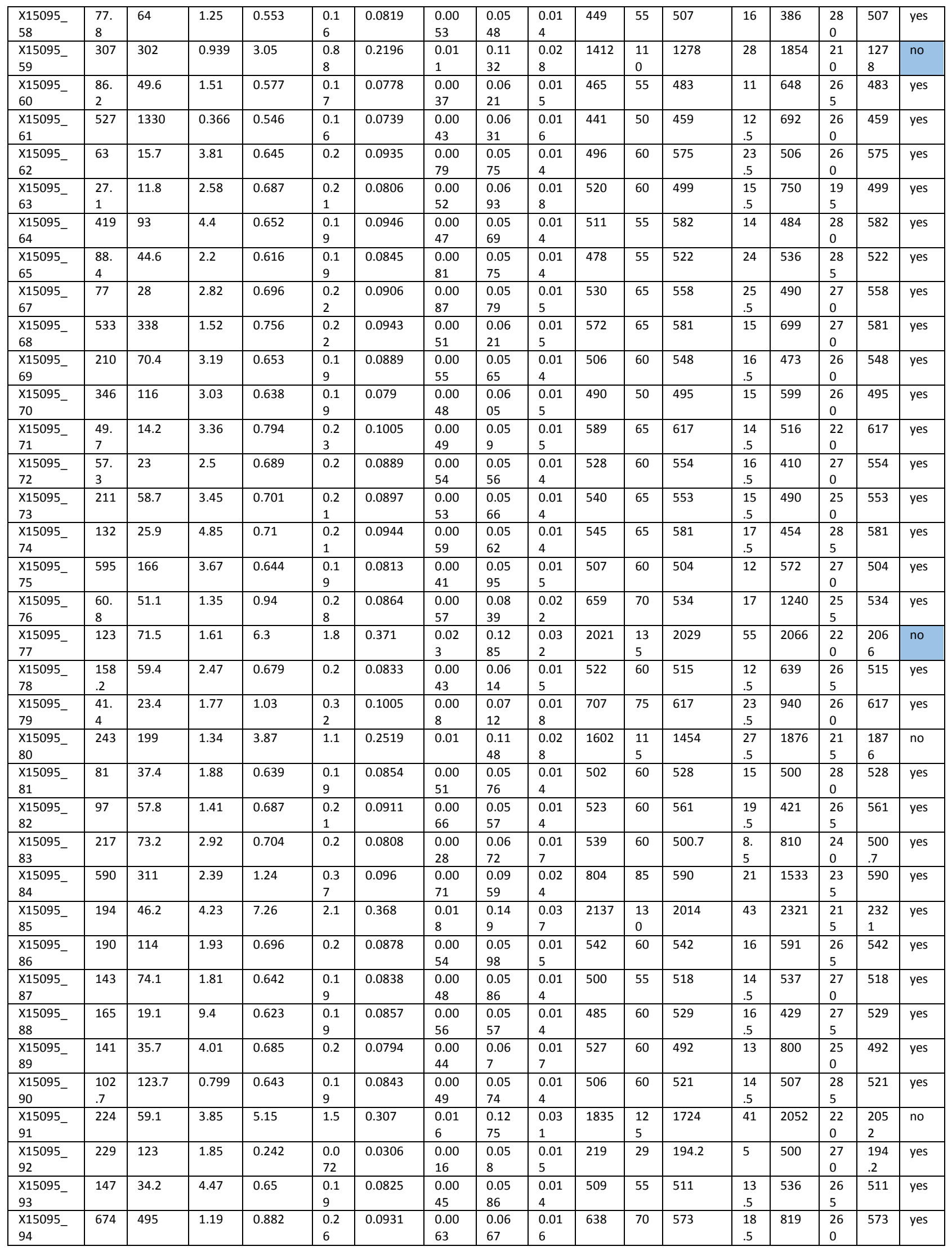




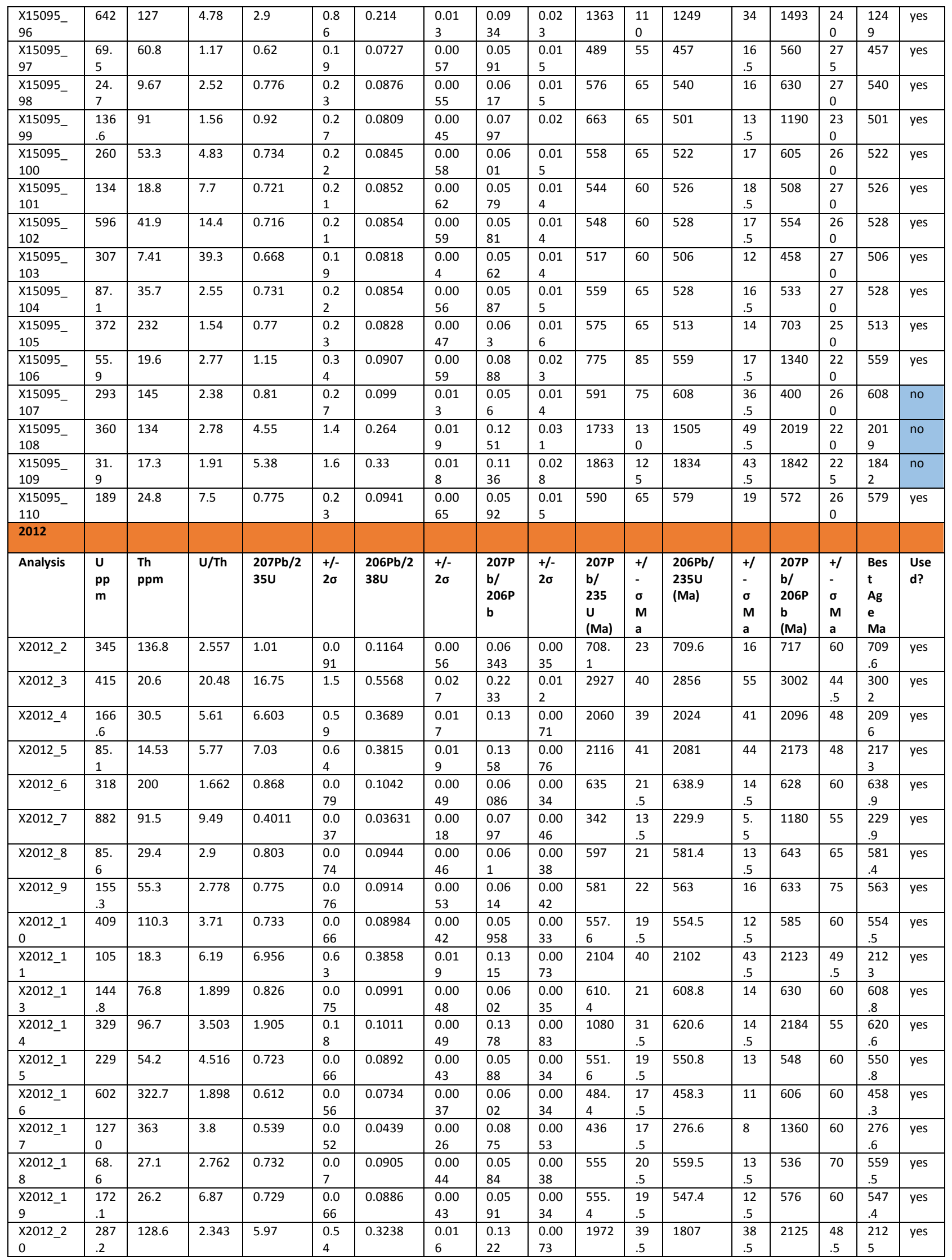




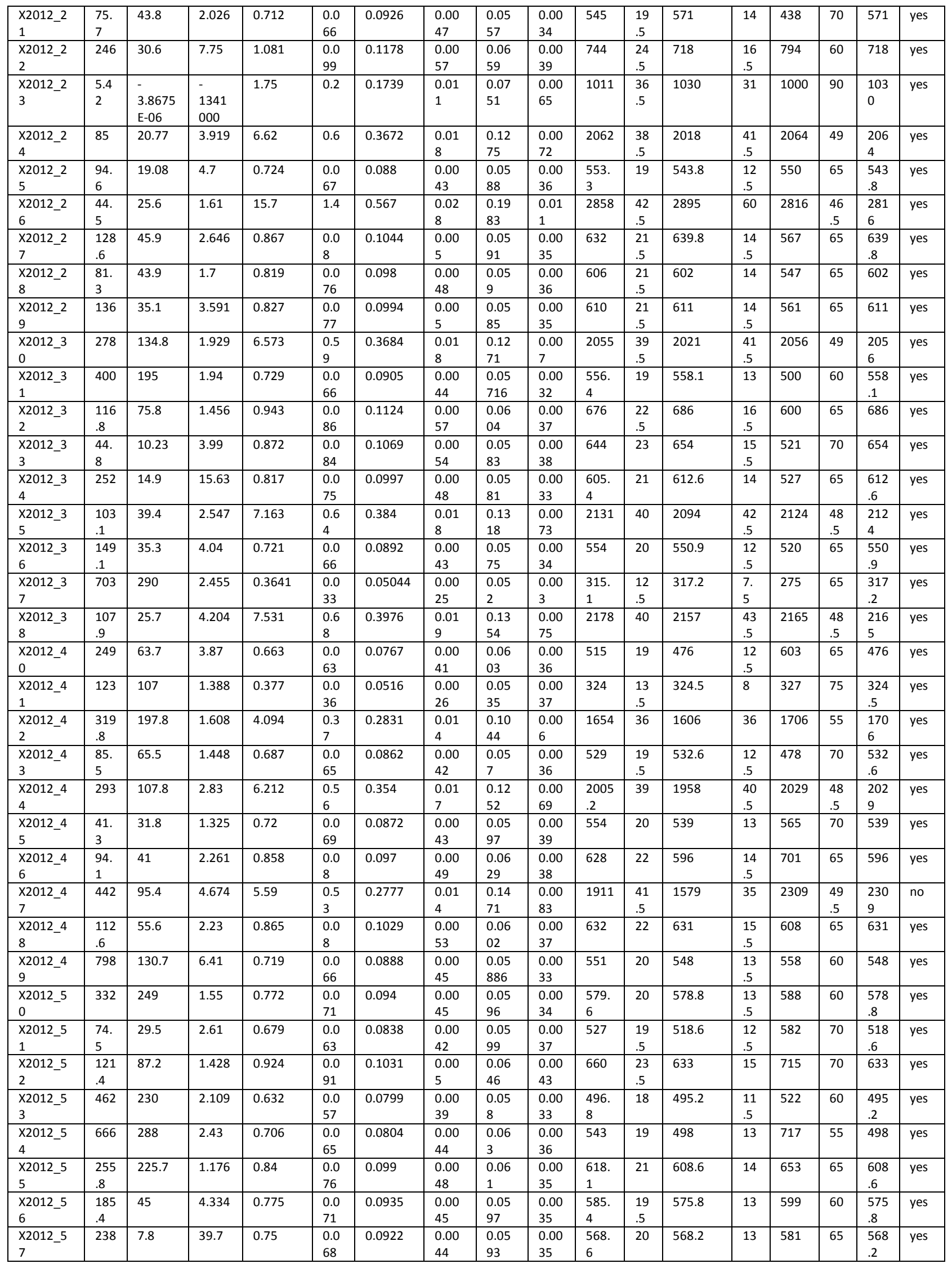




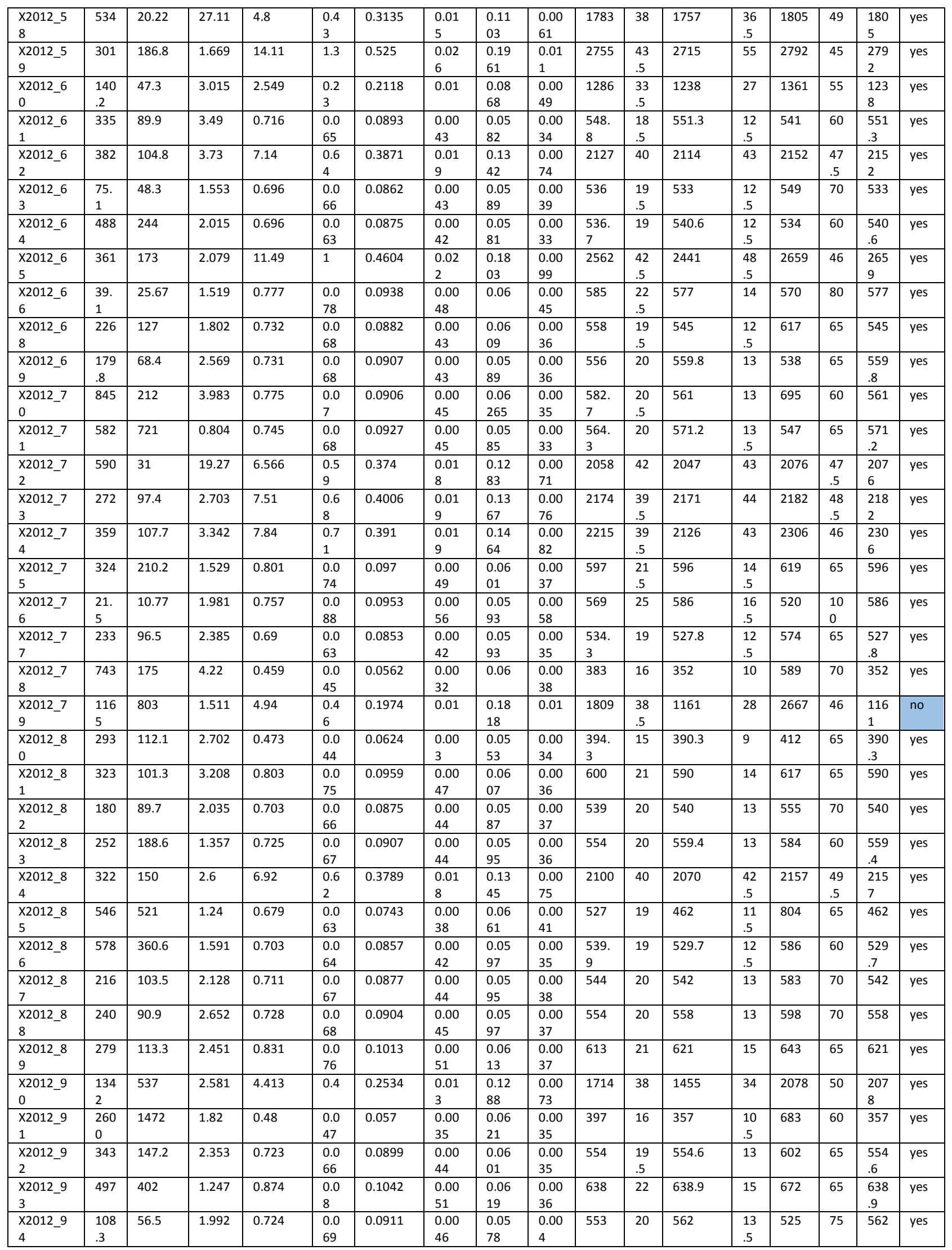




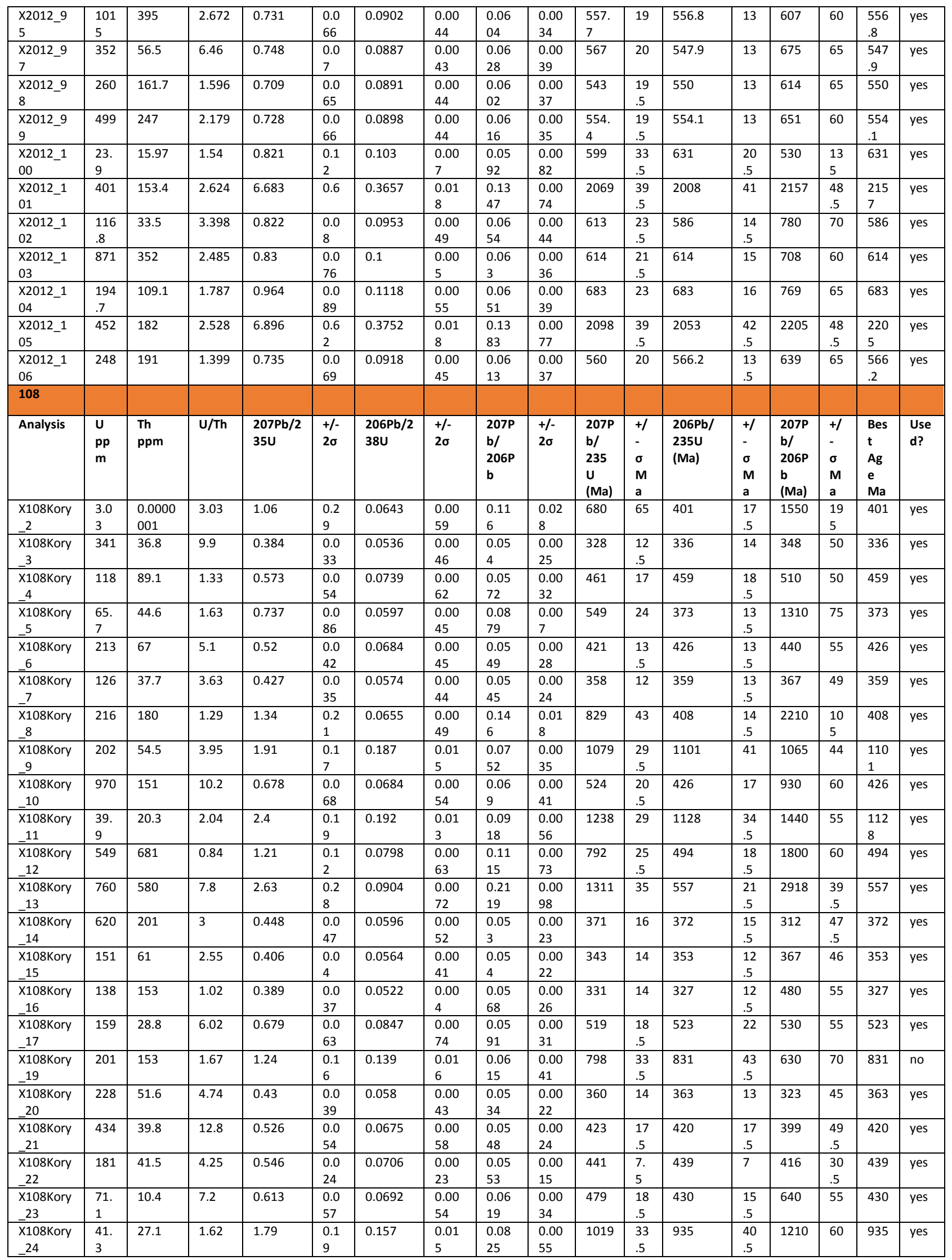




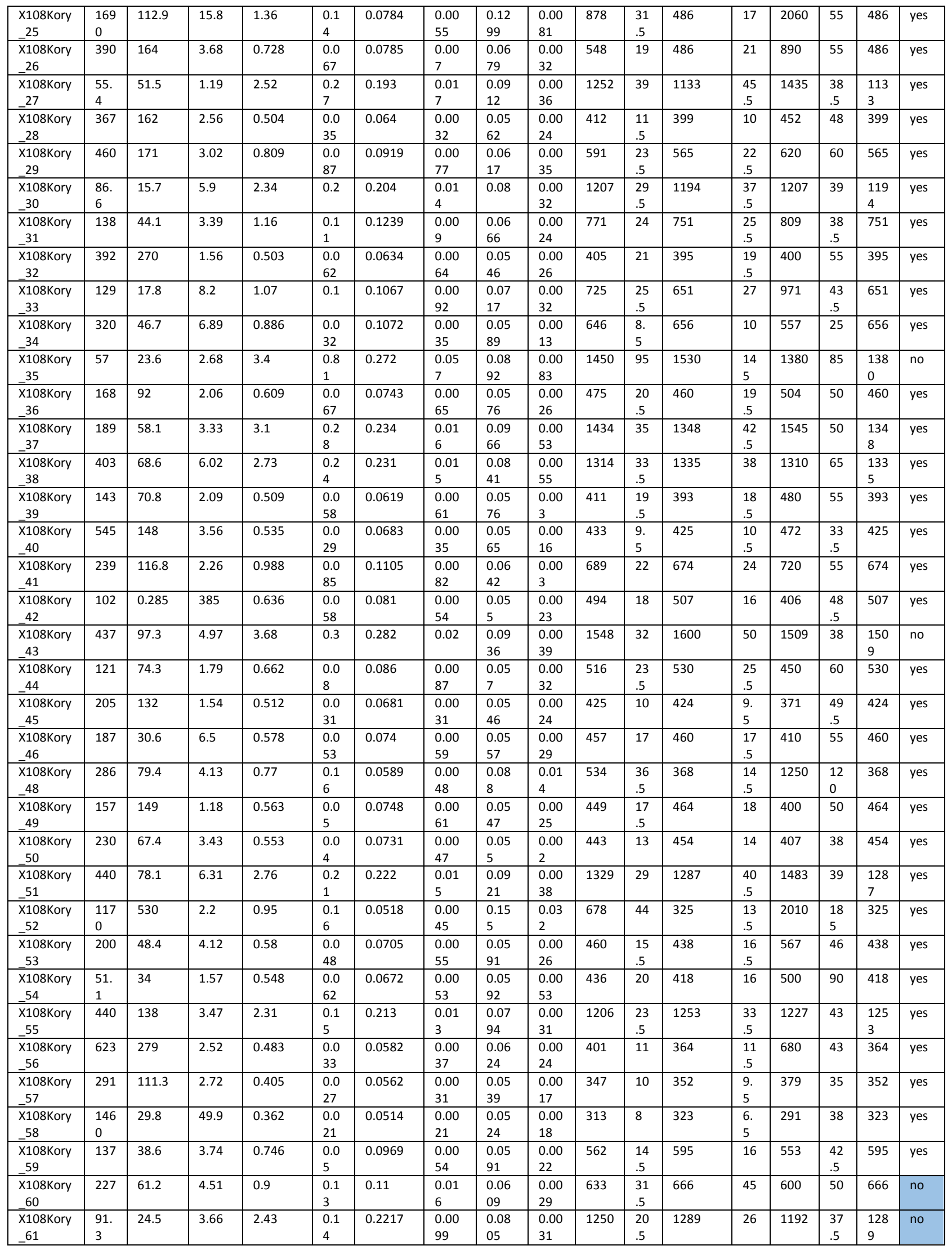




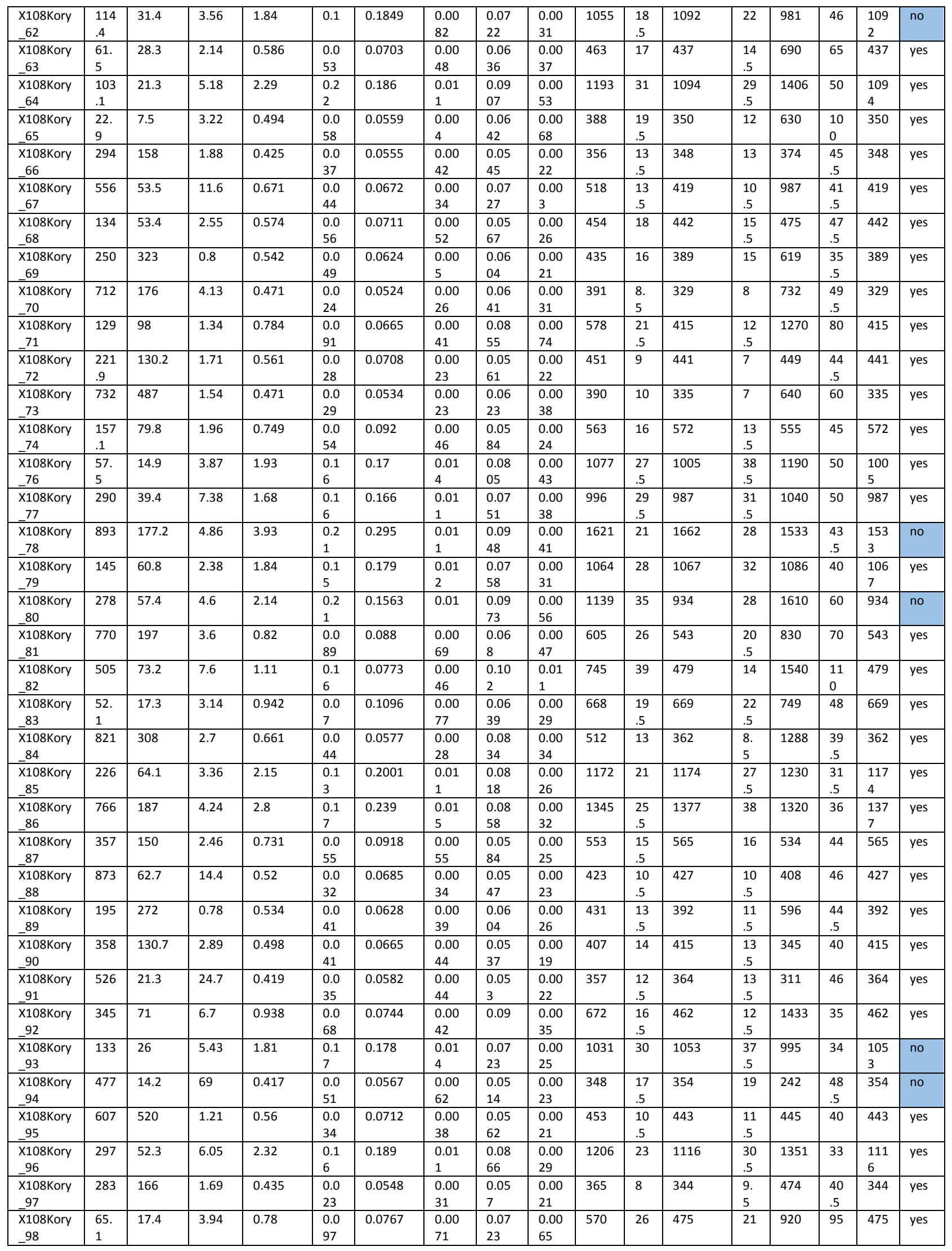




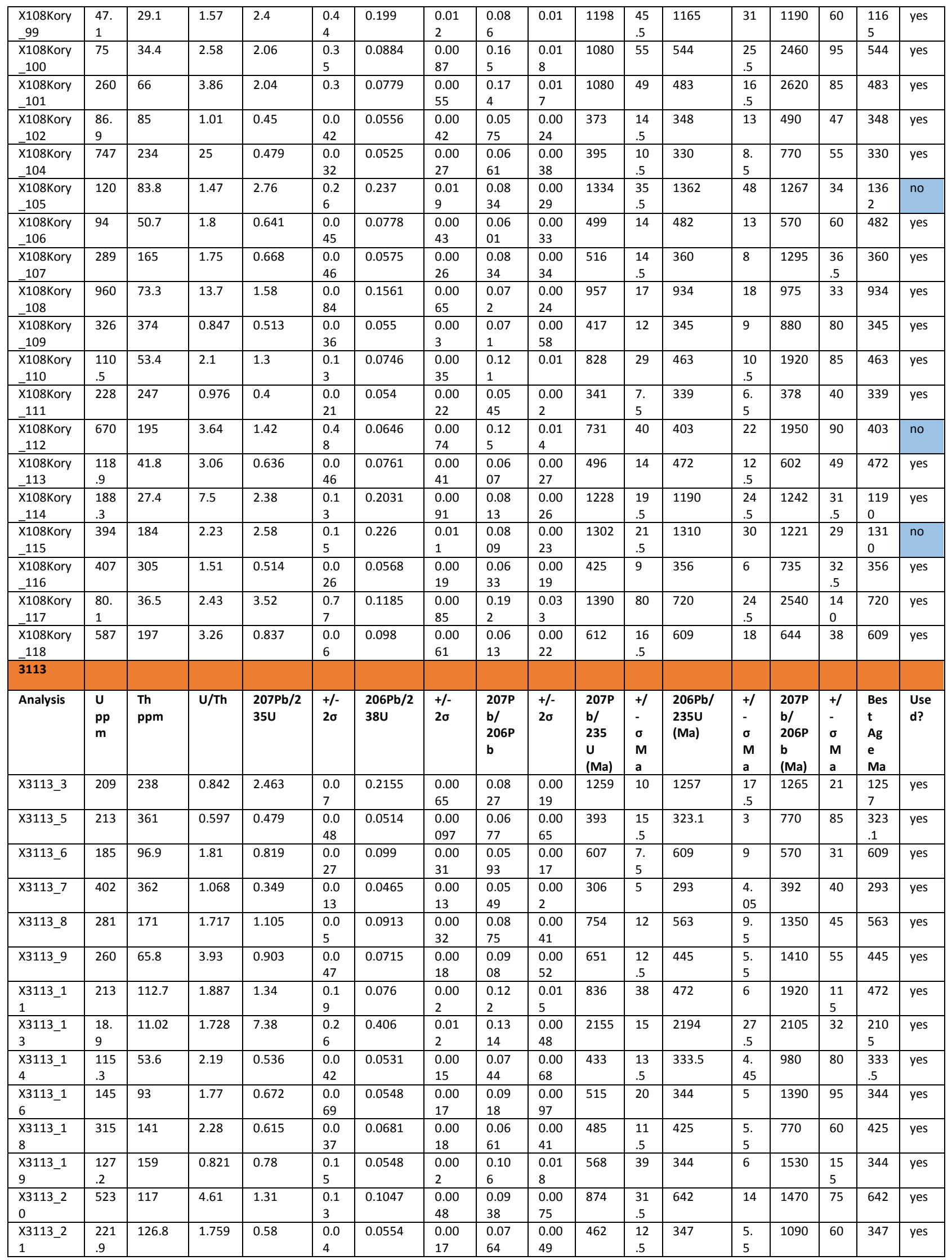




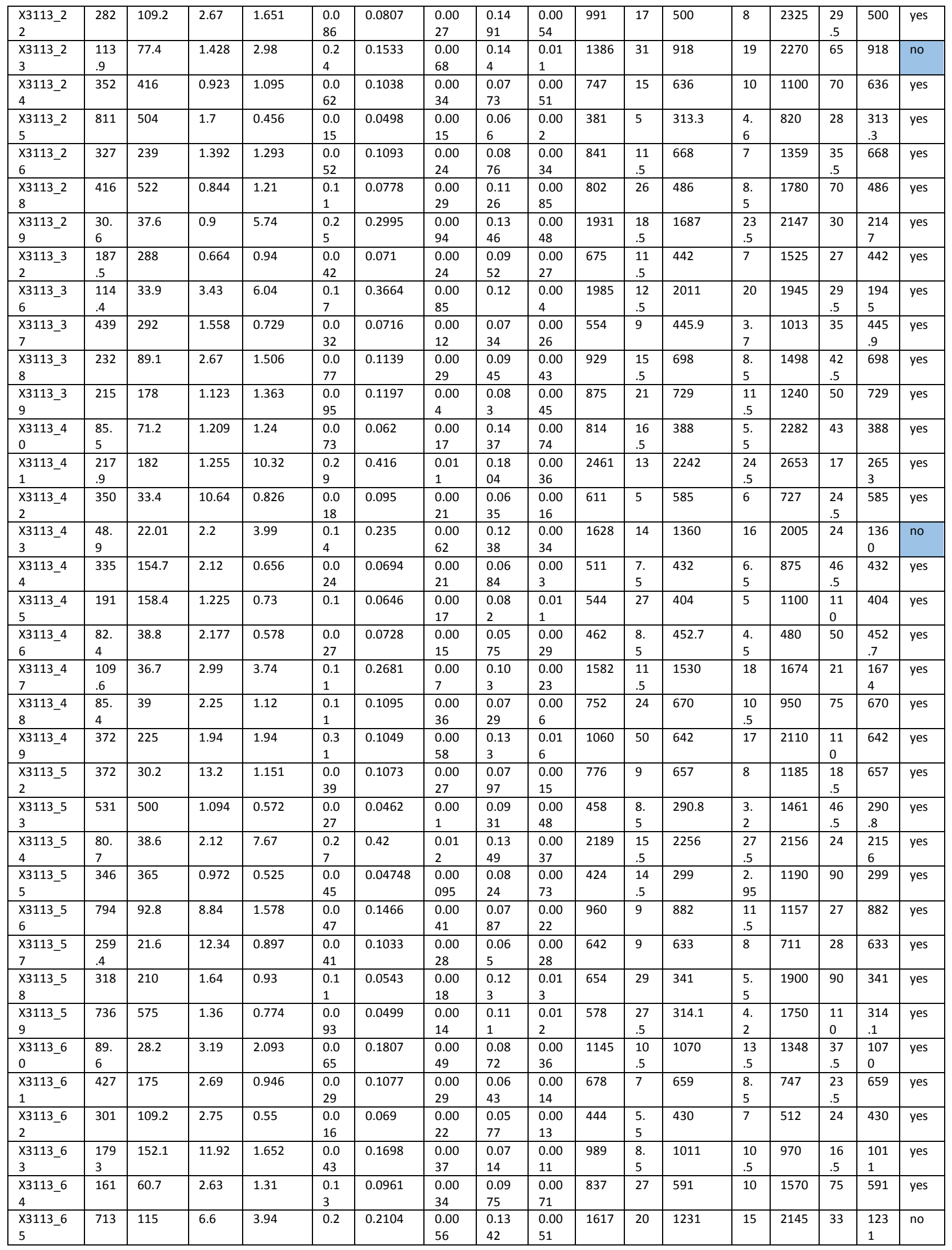




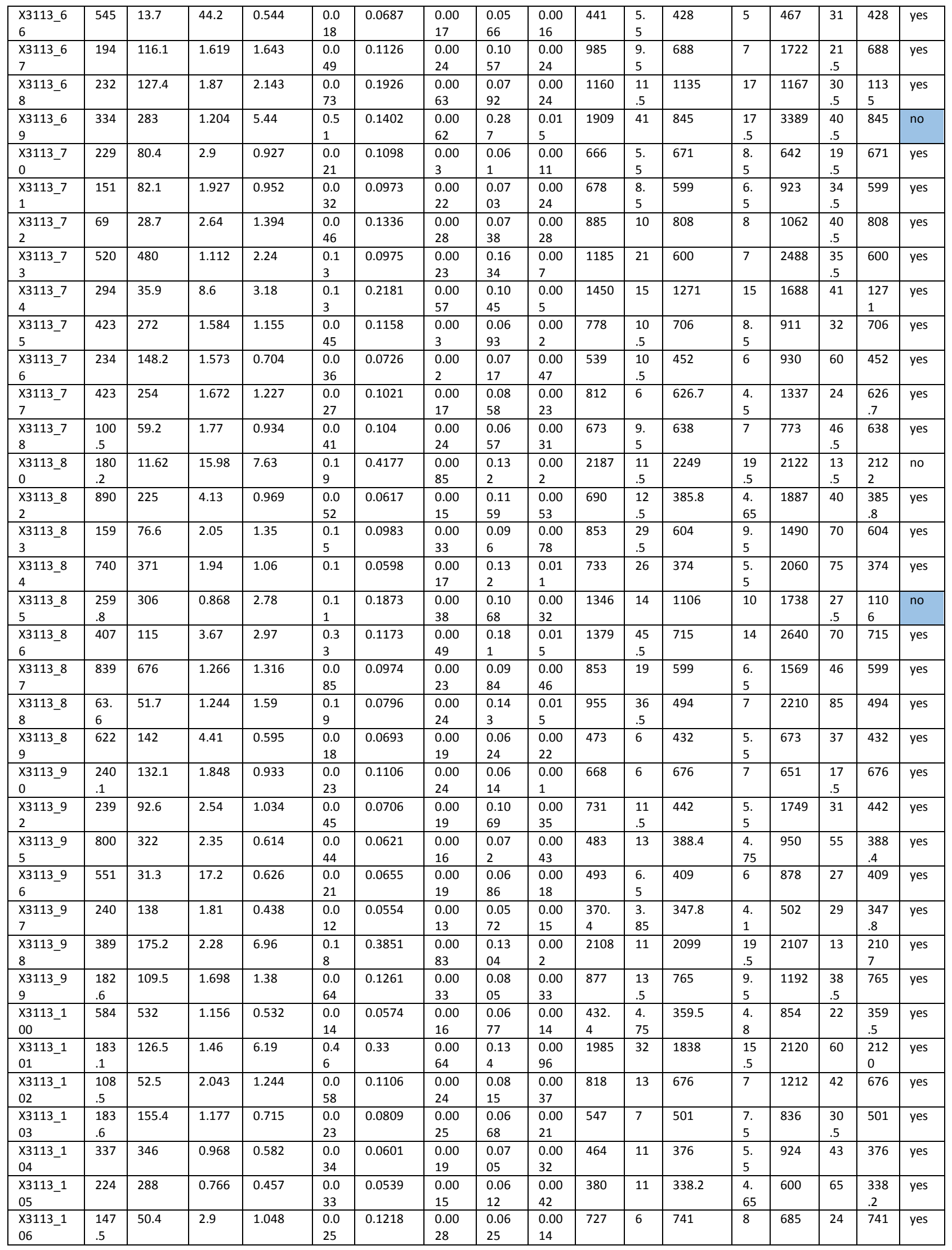




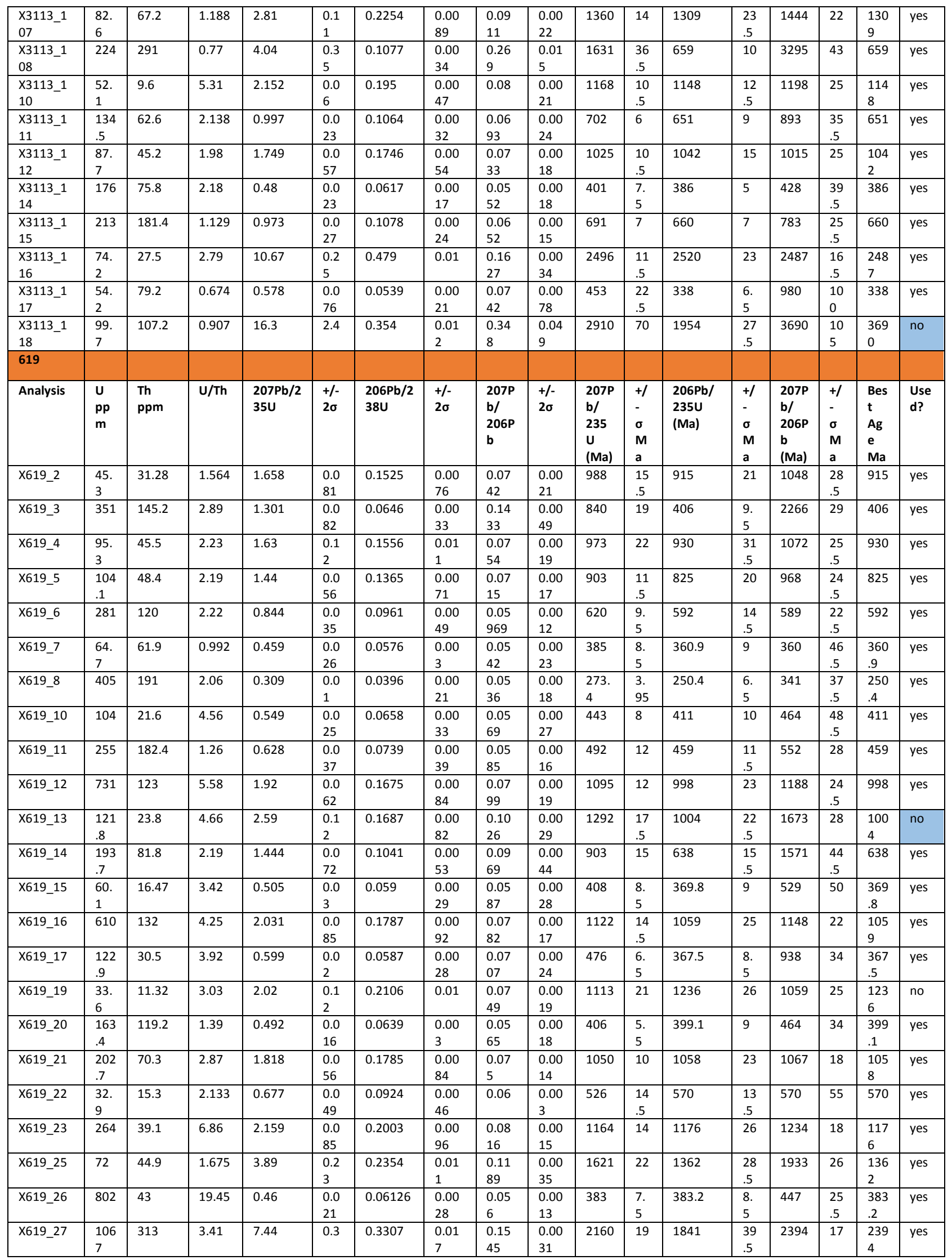




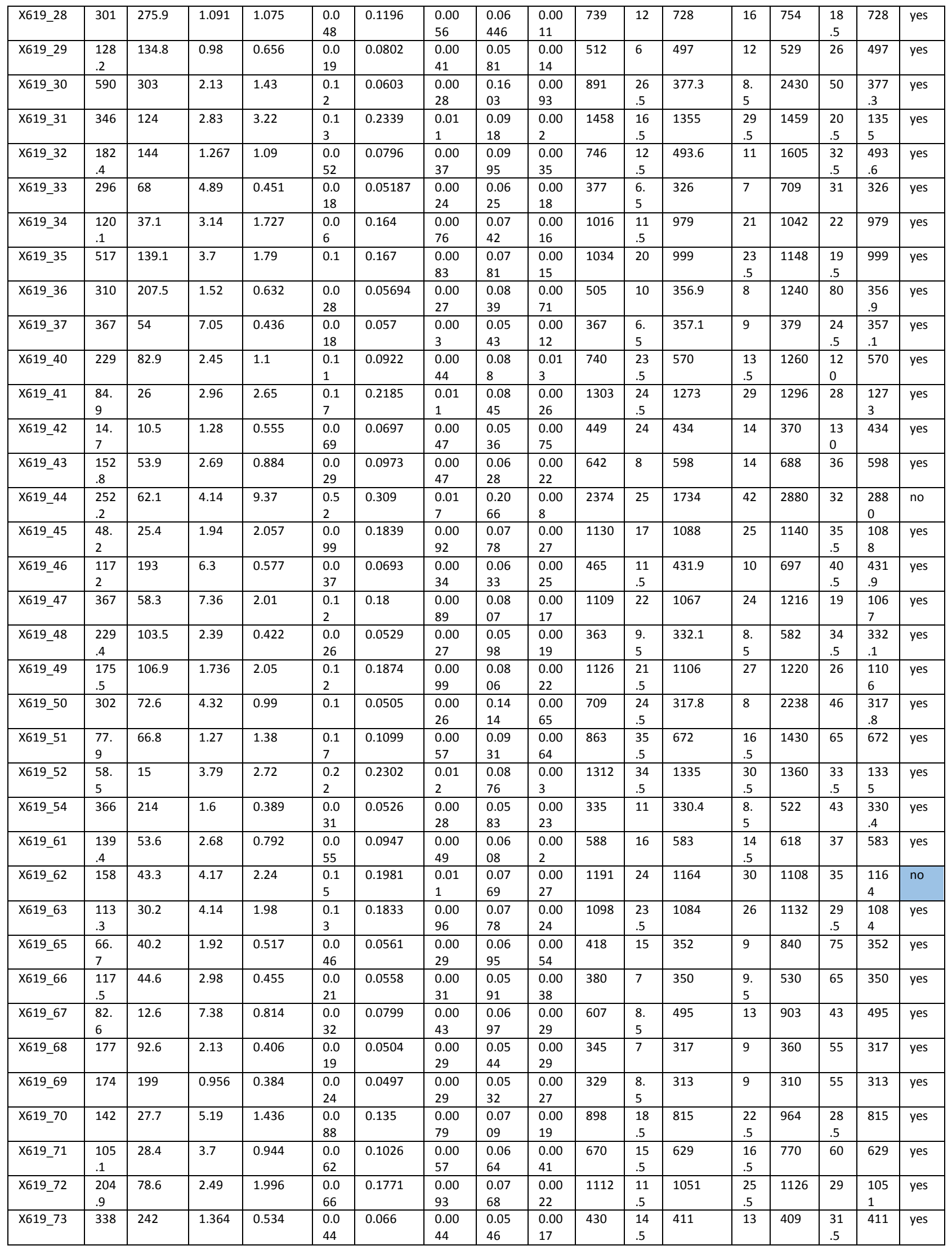




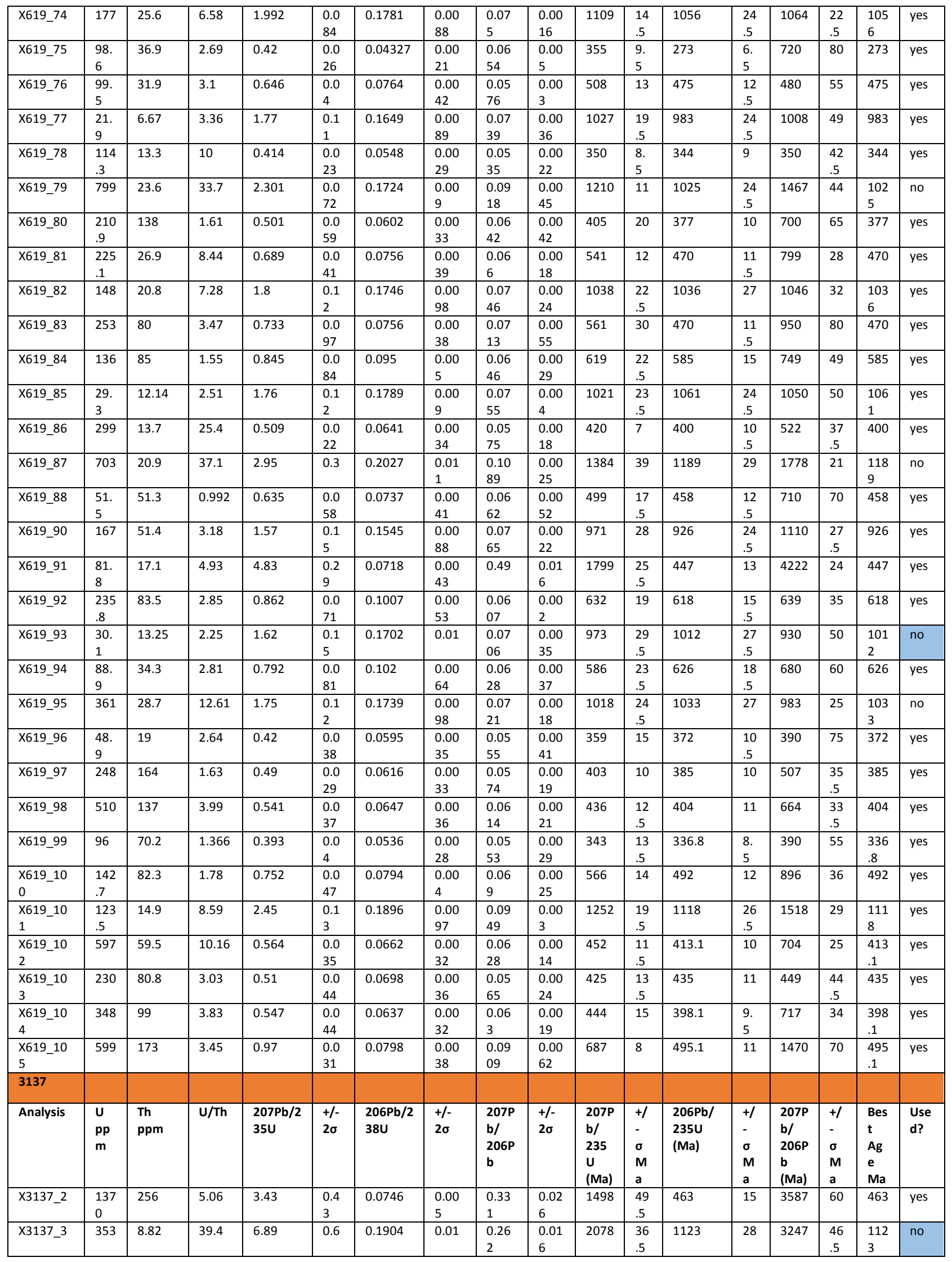




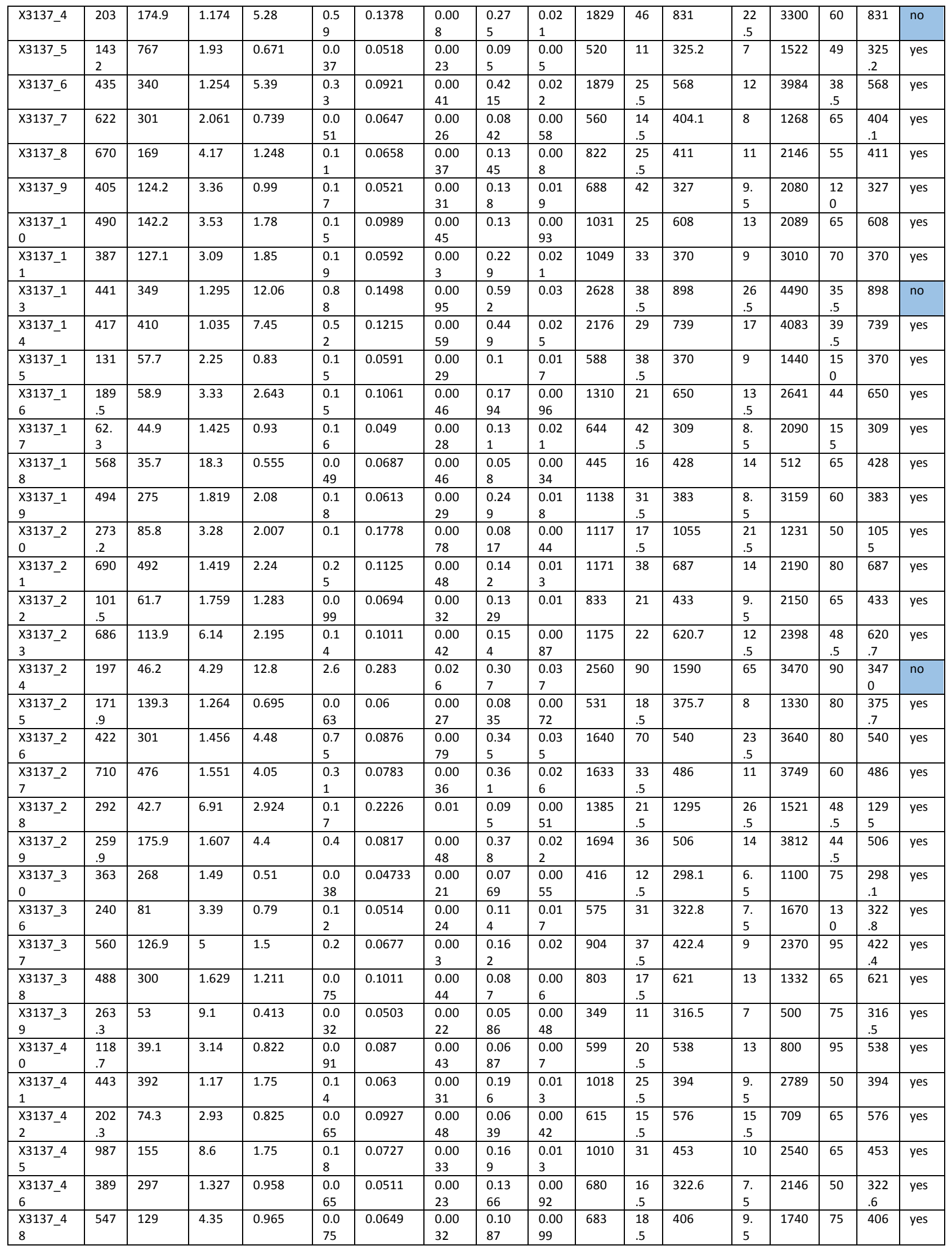




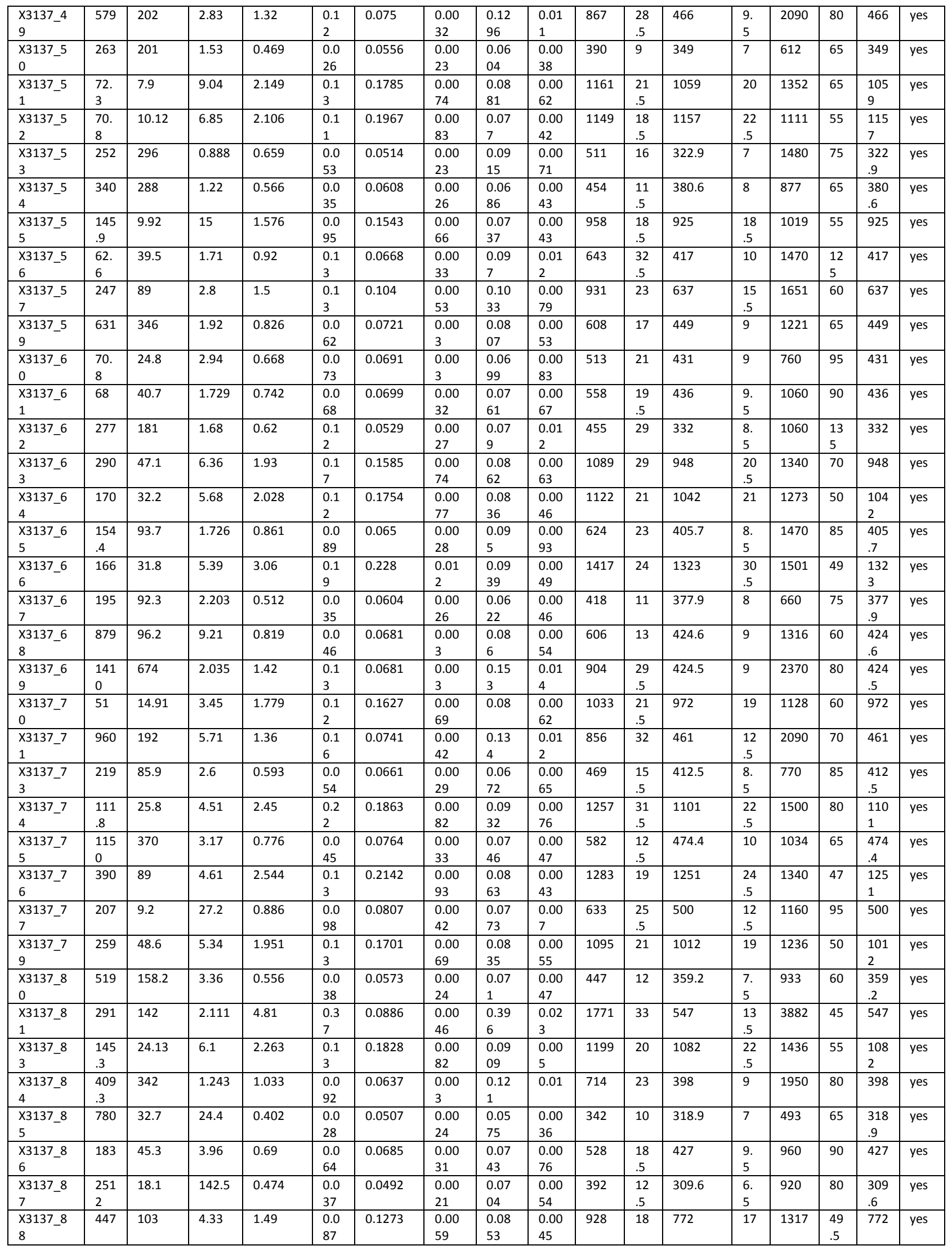




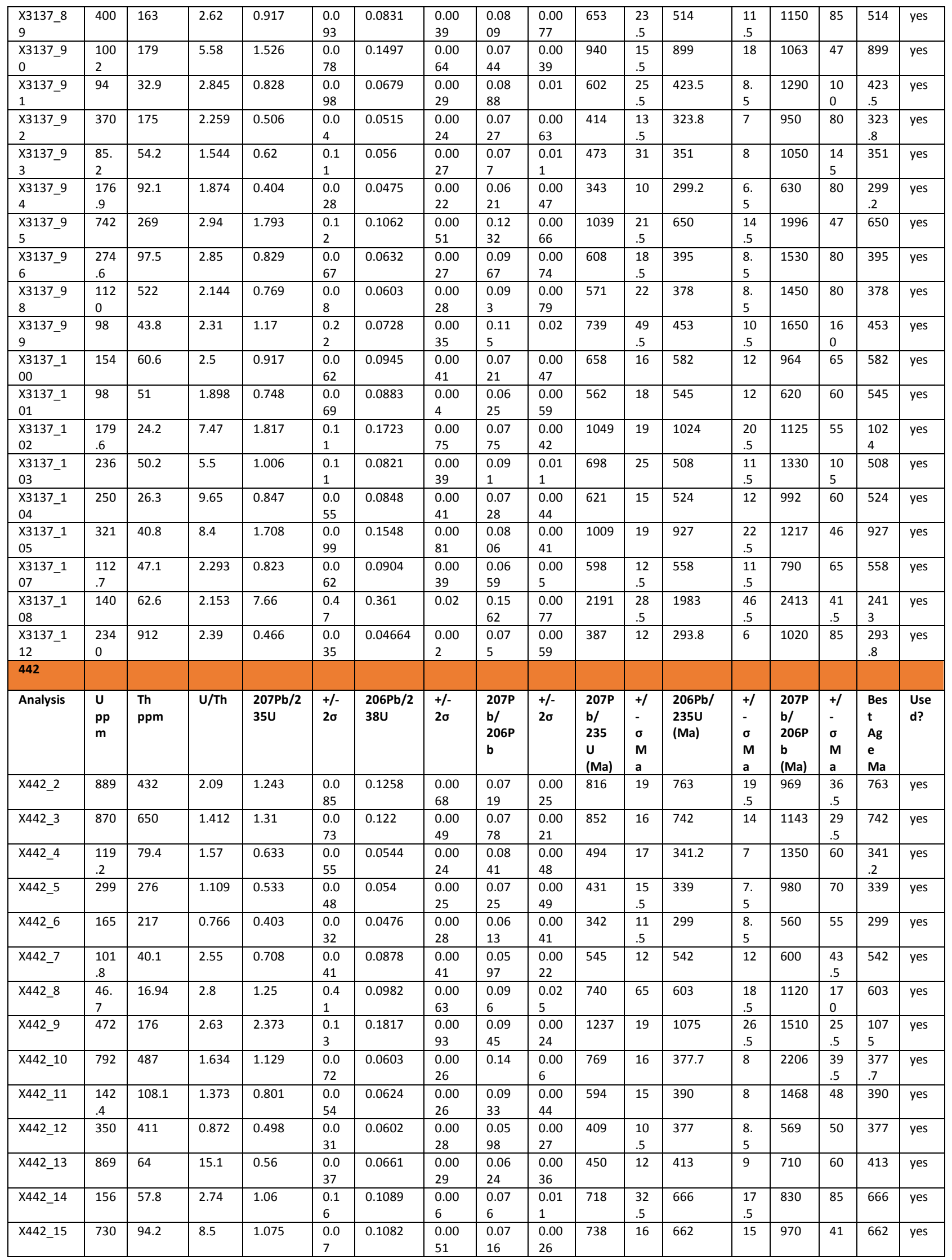




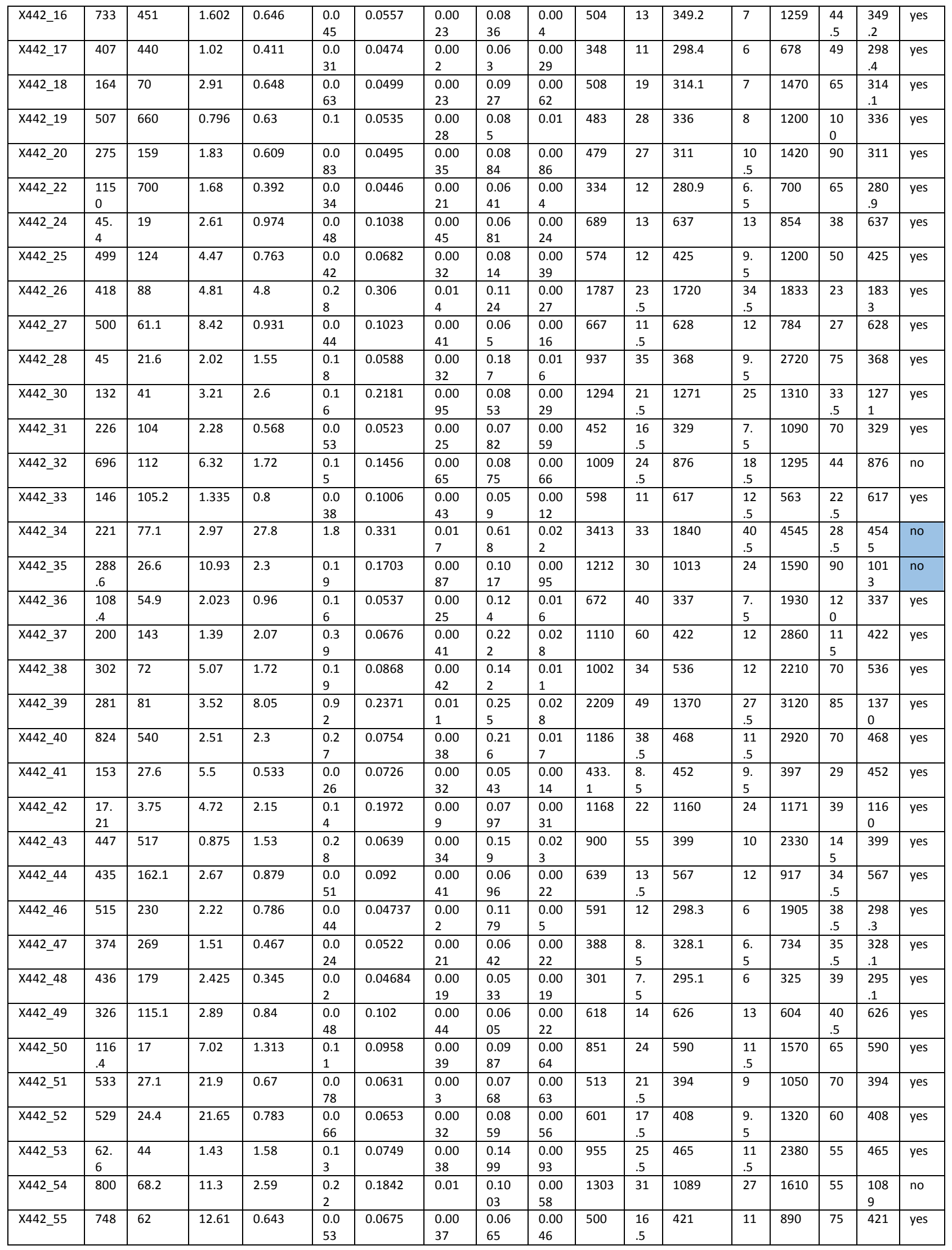




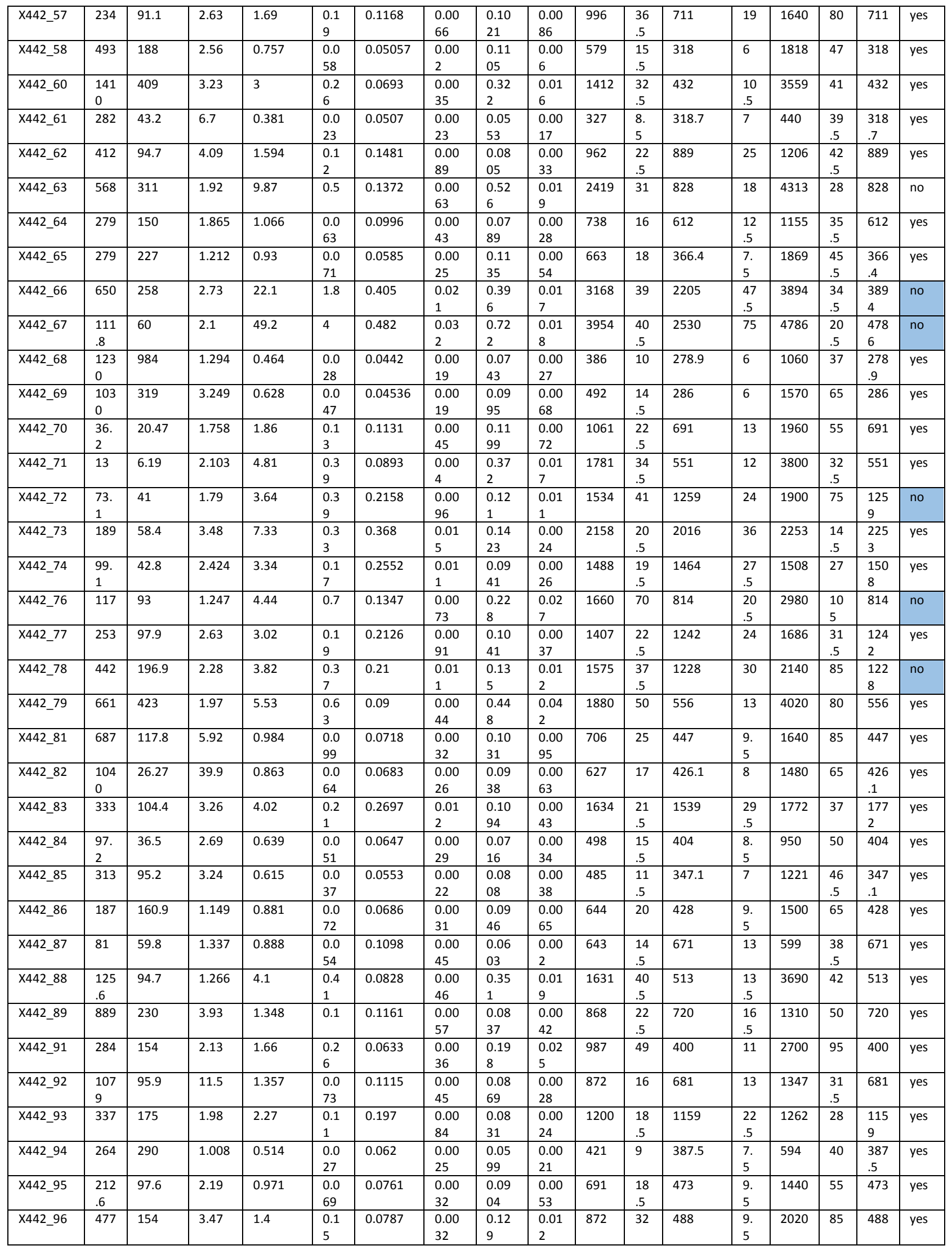




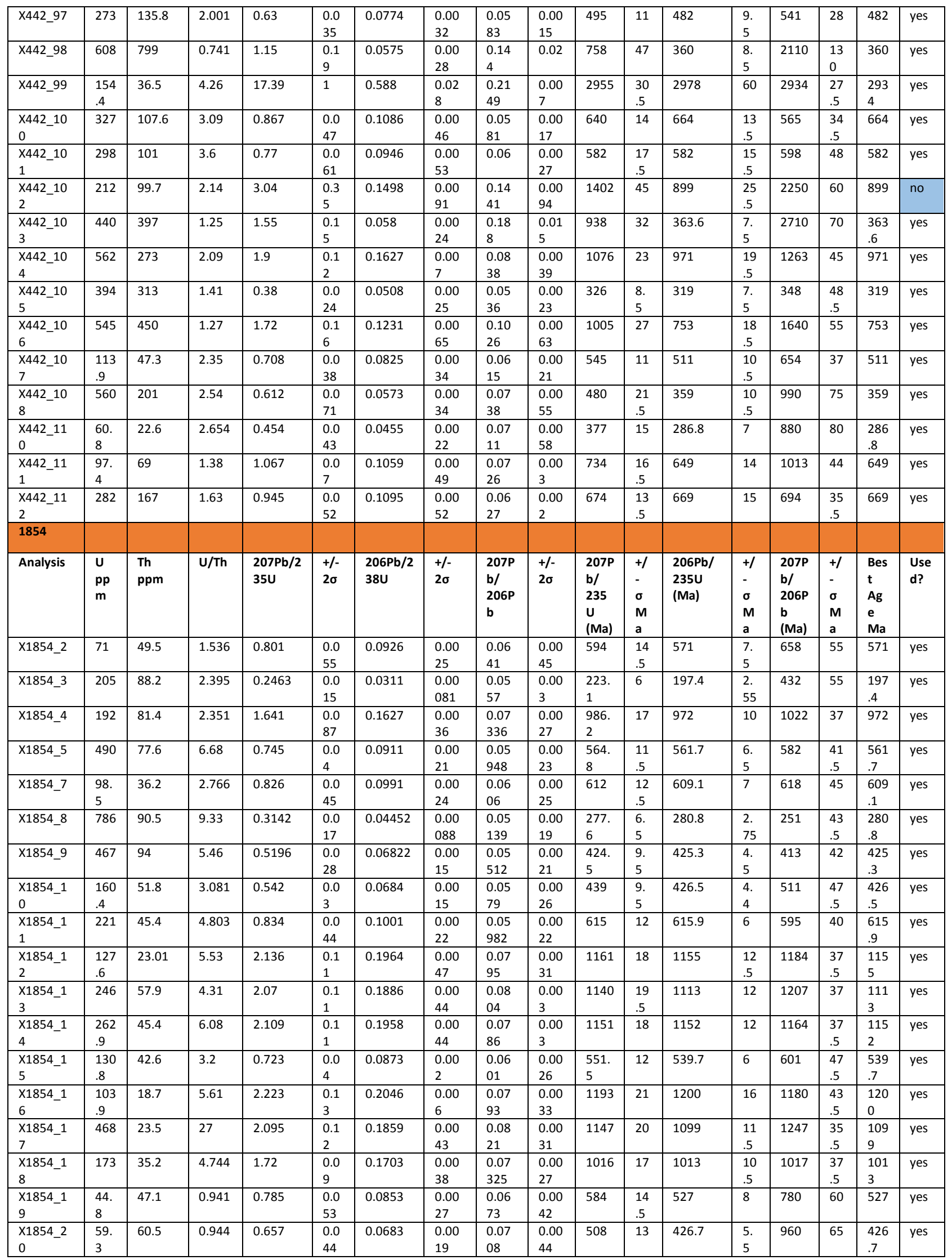




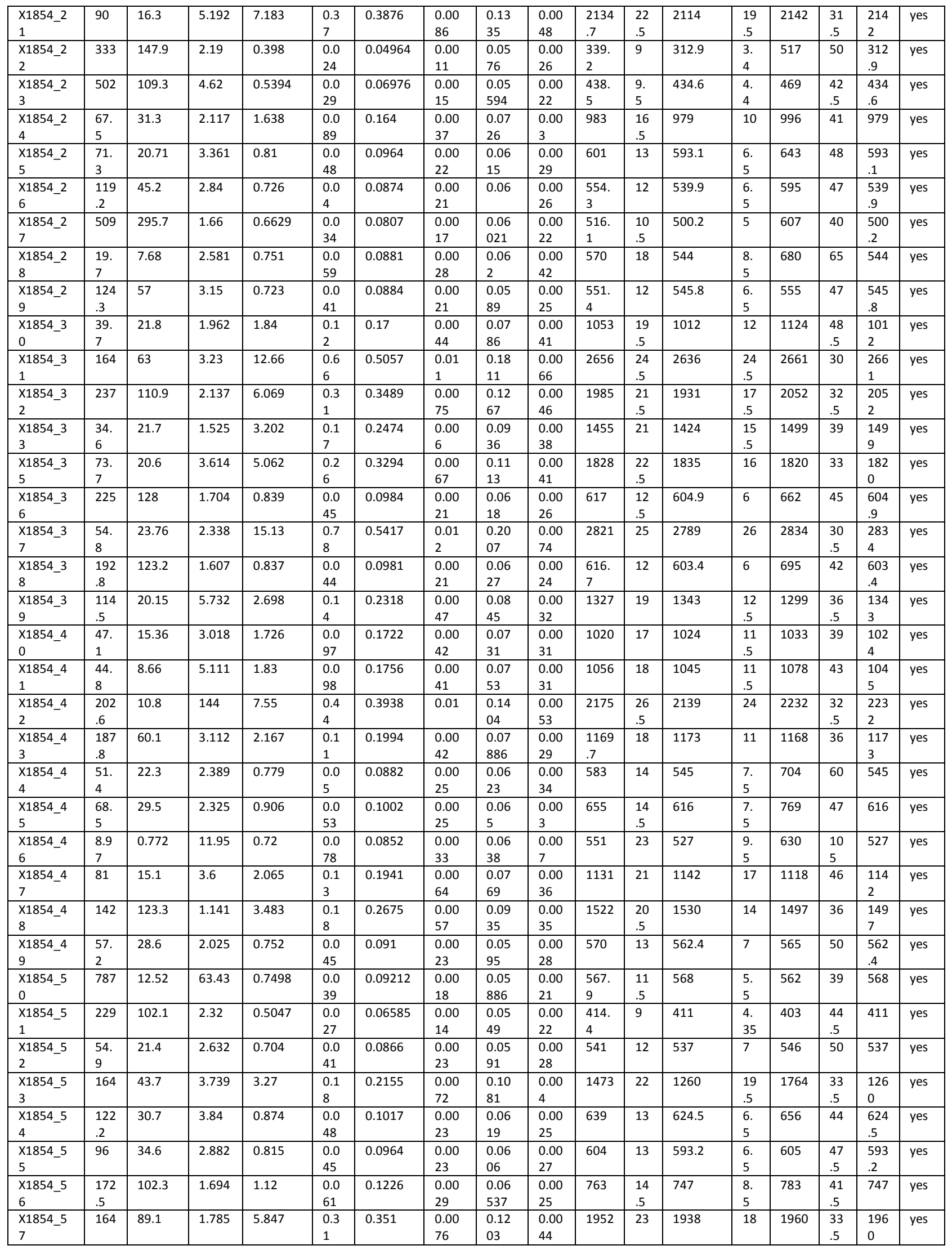




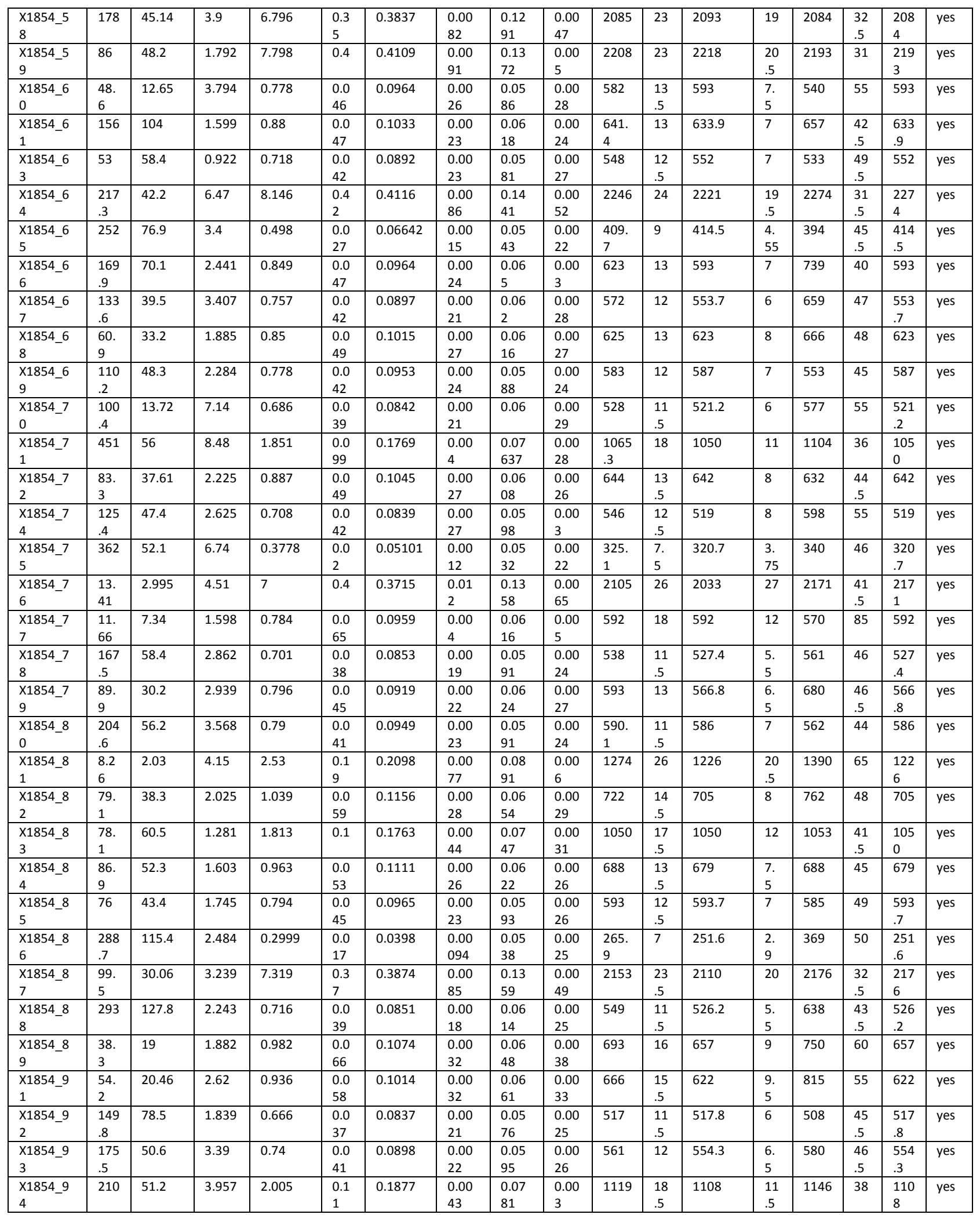




\section{Appendix B: Point Count Data}

Thin Section Point Count

\begin{tabular}{|l|l|l|l|l|l|l|l|l|l|l|}
\hline & Qt & F & L & Qm & Qp & Fp & Fo & Lm & Ls & Lv \\
\hline & & & & & & & & & & \\
\hline & & & & & & & & & & \\
\hline $\mathbf{3 5 7 8 - 1}$ & & & & & & & & & & \\
\hline Points & 339 & 41 & 10 & 324 & 15 & 31 & 10 & 3 & 0 & 7 \\
\hline (Qt+F+L)/400 & .8475 & .1025 & .025 & & & & & & & \\
\hline Percent & 84.75 & 10.25 & 2.5 & & & & & & & \\
\hline
\end{tabular}




\begin{tabular}{|c|c|c|c|c|c|c|c|c|c|c|}
\hline \multirow{2}{*}{\multicolumn{11}{|c|}{$3578-2$}} \\
\hline & & & & & & & & & & \\
\hline Points & 352 & 42 & 6 & 336 & 16 & 40 & 2 & 2 & 0 & 4 \\
\hline$(Q t+F+L) / 400$ & .88 & .105 & .015 & & & & & & & \\
\hline Percent & 88.00 & 10.5 & 1.5 & & & & & & & \\
\hline \multirow{2}{*}{\multicolumn{11}{|c|}{ 966-1 }} \\
\hline & & & & & & & & & & \\
\hline Points & 292 & 94 & 19 & 285 & 7 & 89 & 5 & 2 & 1 & 16 \\
\hline$(\mathrm{Qt}+\mathrm{F}+\mathrm{L}) / 400$ & .655 & .235 & .0475 & & & & & & & \\
\hline Percent & 65.5 & 29.76 & 4.75 & & & & & & & \\
\hline \multirow{2}{*}{\multicolumn{11}{|c|}{$966-2$}} \\
\hline & & & & & & & & & & \\
\hline Points & 362 & 34 & 4 & 337 & 25 & 34 & 0 & 0 & 1 & 3 \\
\hline$(Q t+F+L) / 400$ & .905 & .085 & .01 & & & & & & & \\
\hline Percent & 90.5 & 8.5 & 1.0 & & & & & & & \\
\hline \multicolumn{11}{|l|}{ 966-3 } \\
\hline Points & 379 & 19 & 2 & 367 & 12 & 14 & 5 & 2 & 0 & 0 \\
\hline$(Q t+F+L) / 400$ & .9475 & .0475 & .005 & & & & & & & \\
\hline Percent & 94.75 & 4.75 & .5 & & & & & & & \\
\hline \multicolumn{11}{|l|}{$966-4$} \\
\hline Points & 364 & 30 & 6 & & & & & & & \\
\hline$(Q t+F+L) / 400$ & .91 & .075 & .015 & 340 & 24 & 28 & 2 & 2 & 0 & 4 \\
\hline Percent & 91.0 & 7.5 & 1.5 & & & & & & & \\
\hline \multicolumn{11}{|l|}{788} \\
\hline Points & 335 & 23 & 6 & 331 & 4 & 15 & 8 & 4 & 0 & 2 \\
\hline$(\mathrm{Qt}+\mathrm{F}+\mathrm{L}) / 400$ & .8375 & .0575 & .015 & & & & & & & \\
\hline Percent & 83.75 & 5.75 & 1.5 & & & & & & & \\
\hline \multicolumn{11}{|l|}{15656} \\
\hline Points & 366 & 31 & 3 & 365 & 1 & 25 & 6 & 0 & 0 & 3 \\
\hline$(\mathrm{Qt}+\mathrm{F}+\mathrm{L}) / 400$ & .915 & .0775 & .0075 & & & & & & & \\
\hline Percent & 91.5 & 7.75 & .75 & & & & & & & \\
\hline \multicolumn{11}{|l|}{ 2012-1 } \\
\hline Points & 380 & 12 & 8 & 364 & 16 & 12 & 0 & 4 & 4 & 0 \\
\hline$(\mathrm{Qt}+\mathrm{F}+\mathrm{L}) / 400$ & .95 & .03 & .02 & & & & & & & \\
\hline Percent & 95.0 & 3.0 & 2.0 & & & & & & & \\
\hline & & & & & & & & & & \\
\hline \multicolumn{11}{|l|}{ 2012-2 } \\
\hline Points & 374 & 26 & 0 & 353 & 21 & 24 & 2 & 0 & 0 & 0 \\
\hline
\end{tabular}




\begin{tabular}{|l|l|l|l|l|l|l|l|l|l|l|l|}
\hline$(\mathrm{Qt}+\mathrm{F}+\mathrm{L}) / 400$ & .935 & .065 & 0 & & & & & & & \\
\hline Percent & 93.5 & 6.5 & 0 & & & & & & & \\
\hline & & & & & & & & & & \\
\hline $\mathbf{1 2 5 4 2}$ & & & & & & & & & & \\
\hline Points & 208 & 188 & 3 & 205 & 3 & 89 & 99 & 3 & 0 & 0 \\
\hline (Qt+F+L)/400 & .52 & .471 & .008 & & & & & & & \\
\hline Percent & 52.0 & 47.1 & .8 & & & & & & & \\
\hline & & & & & & & & & & \\
\hline & & & & & & & & & & \\
\hline & & & & & & & & & & \\
\hline & & & & & & & & & & \\
\hline
\end{tabular}

Qt= Quartz, F= Feldspar, L=Lithics, Qm=Quartz monocrystalline, $Q p=Q u a r t z$

polycrystalline, Fp=Plagioclase Feldspar, Fo/m=Orthoclase Feldspar, Lm=Metamorphic

Lithics, Lv=Volcanic Lithics, Ls=Sedimentary lithics.

\section{Appendix C: Thin Section Descriptions}

\section{Thin Sections: South Florida}

The table of thin section data from southern Florida is displayed in Table 4. Sample descriptions are provided below. Coarse- grained samples were point counted and described using the Folk (1980) classification scheme (Figure 50). Several samples from South Florida were too finegrained to point-count. 
$\underline{\text { ODP }}$

Although no zircons were obtained from the clastic interval of the ODP wells, two thinsections were made. No thin-sections were made for the basement section due to the small sample size, and to increase the chances of getting a usable number of zircons for dating.

Sample 1 was collected from ODP Site 0537 from Core 13R, section 1, and interval 95110 (Figure 51). It is interpreted as arkosic sandstone conglomerate with calcite cement. The sandstone consists primarily of quartz and volcanic fragments (perthite) with calcite cement. Quartz grains are large (up to $1 \mathrm{~mm}$ ), and well rounded. Inclusions of zircon or tourmaline are present in several quartz grains. Calcite cements are sparry with a second phase of blocky calcite cement.

Sample 2 was collected from ODP site 77 hole 0538A, 034R, 01W, interval 54.57-57 (Figure 51). It is interpreted as diabase from Deep Sea Drilling report volume 77. It consists of fine-grained diabase with plagioclase, biotite, green clays, and opaque minerals. Quartz veins are common, and occur at three different angles. En echelon fractures may be present. Ribbons of quartz are interpreted as interstitial quartz, which formed during mild degrees of mylonitization (Deep Sea Drilling report, volume 77).

$\underline{778}$

Two samples were examined from 778. Sample 778-1 consists of cuttings, and was not point-counted (Figure 52). The sample came from the mixed shale/sandstone/dolomite/limestone section. It consists mainly of shale and calcite, as well as chambered fossils. Blocky calcite cements and quartz fragments are also common.

Sample 778-2 was point counted (Figure 52). It was collected from the lower clastic section of the Wood River. Sample 778-2 consists primarily of angular-subrounded quartz 
fragments. Grain size is fairly unimodal at around $.5 \mathrm{~mm}$. Minor twinned feldspar and orthoclase/microcline is also present. Lithic fragments are extremely uncommon, although some volcanic fragments may be present.

10566

Sample 10566 was pointed counted (Figure 52). It consists primarily of quartz and feldspar fragments. Quartz fragments are well rounded and fairly unimodal $(.25 \mathrm{~mm})$. Lithic fragments are rare, aside from some perthite.

$\underline{1655}$

No zircons were obtained from sample 1655. Sample 1655 was not point- counted. The sample comes from the upper mixed limestone/shale mix of the Wood River/proximal clastics. Sample 1655 consists mostly of mixed shale with quartz and ooid fragments. Carbonate fragments consist of mud and ooids. The matrix is composed of blocky calcite cement.

$\underline{966}$

No zircons were collected from 966 but four thin-sections were point counted (Figure 52). Sample 966 consists mainly of sub-rounded quartz fragments and twinned/untwined feldspar. Metamorphic fragments are mainly quartzite. Volcanic fragments include perthite. Porosity is fairly low (less than 5\%). Some porosity may be secondary from feldspar dissolution. Grain size is somewhat unimodal $(.1-.5 \mathrm{~mm})$. The sample is moderately sorted. $\underline{2012}$

Three thin-sections were point-counted for sample 2012. It consists mainly of quartz, with some feldspar fragments (Figure 52). Lithic fragments are rare. Sample 2012 ranges from 
an arenite to arkose sandstone. Some lithic fragments are present. The sample is fairly porous ( $\sim 8 \%$, but becomes more compact towards the base of the section. Thin- sections collected at the base is composed of metamorphic quartzite.

$\underline{12542}$

No zircons were obtained from sample 12542 (Figure 53). The sample consists of detrital quartz $(40 \%)$ and feldspars and other volcanic fragments ( $60 \%)$. Feldspars are very common. Perthites typically have stained plagioclase cored, with mixed plagioclase/orthoclase exteriors. For the purpose of this study, perthites were counted as igneous fragments. Feldspars appear to have dissolved, forming secondary porosity. The abundance of perthite indicates a igneous source with close proximity to a granitic source.

$\underline{3578}$

Three thin-sections were collected from 3578, one of which was composed of shale and was not point-counted. The shale sample consists mainly of mud and fine detrital quartz and muscovite. Sandy intervals consisted mainly of feldspars and quartz. Feldspar is twinned and in some locations appears to have been partially dissolved. Detrital mica is also present. Lithics consist primarily of sandstone/shale fragments, and possibly metamorphic fragments as well. Grains are soubrounded-subangular, and well sorted. Porosity is fairly low (less than 5\%), and compacted quartz grains are common. Quartz cements are also present.. 


\section{Thin Sections South Georgia Rift}

Table of thin-sections from the South Georgia Rift is displayed in Table 2. All samples from the South Georgia Rift collected were too fine-grained for point counting, but observations were recorded on grain- size and composition.

Sample 442

Sample 442 includes 442-1 and 442-2, which are composed mainly of quartz but also abundant muscovite and feldspar. Sample 442-1 is composed primarily of, with alternating laminae of fine- to medium-grained, poorly sorted, subangular quartz, and fine-grained, dark mudstone. Quartz grains range from .05-.15 mm (Figure 54). The grain size of quartz increases from layer to layer and although the up direction is not known, the sequence likely represents a coarsening or fining upward sequence. Possible fossils include bivalves, and chambered gastropods.

\section{Sample 108}

Sample 108 is composed mainly of detrital quartz and micas (Figure 54). Quartz fragments are subangular, and less than $0.1 \mathrm{~mm}$ in diameter. The matrix is composed of mudsized particles. Abundant unknown opaque grains are present. Small calcite veins are present.

\section{Sample 619}

Sample 619 is shale composed mainly of mud-sized particles (Figure 54). A thin layer of fine-grained quartz and mica is present, but not prevalent throughout the sample. Grains are poorly sorted, and separated by mud matrix. Veins of calcite are also present.

\section{Sample 3137}


Sample 3137 was described from two thin-sections. It is composed mainly of black shale that is composed of fine mud (Figure 54). Detrital composition consists predominantly of quartz $(.15 \mathrm{~mm})$, feldspar laths, and muscovite. Muscovite is the most abundant detrital mineral ( 60\%) Quartz fragments are poorly sorted and subangular. Micas are small, and fibrous. Calcite fragments are also present. 